DANILO ZUCOLLI FIGUEIREDO

Discrete-Time Jump Linear Systems with Markov Chain in a General State Space

São Paulo 


\section{Discrete-Time Jump Linear Systems with Markov Chain in a General State Space}

Tese apresentada à Escola Politécnica da Universidade de São Paulo para obtenção do título de Doutor em Ciências

Área de Concentração:

Engenharia de Sistemas

Orientador:

Prof. Dr. Oswaldo Luiz do Valle Costa

São Paulo 
Este exemplar foi revisado e corrigido em relação à versão original, sob responsabilidade única do autor e com a anuência de seu orientador.

São Paulo, de de

Assinatura do autor:

Assinatura do orientador:

\section{Catalogação-na-publicação}

Figueiredo, Danilo Zucolli

Discrete-time jump linear systems with Markov chain in a general state space / D. Z. Figueiredo -- versão corr. -- São Paulo, 2016.

$141 \mathrm{p}$.

Tese (Doutorado) - Escola Politécnica da Universidade de São Paulo. Departamento de Engenharia de Telecomunicações e Controle.

1.Sistemas lineares 2.Controle estocástico 3.Controle ótimo 4.Cadeias de Markov 5.Equações de Riccati I.Universidade de São Paulo. Escola Politécnica. Departamento de Engenharia de Telecomunicações e Controle II.t. 
À memória de meus avós, Luis e Anna, Herminio e Lorenzina 


\section{Acknowledgments (Agradecimentos)}

Ao Prof. Dr. Oswaldo Luiz do Valle Costa, pela oportunidade de realizar este trabalho, pelos valiosos ensinamentos e pelo exemplo de competência acadêmica.

À banca examinadora, pela análise e por suas contribuições. Agradeço especialmente aos professores Dr. Ricardo Paulino Marques e Dr. Michael Viriato Araujo, que compuseram a banca de meu exame de qualificação, pelo incentivo e orientações sobre o rumo de minhas pesquisas.

Aos docentes do programa de pós-graduação da Escola Politécnica, em especial, aos professores do Laboratório de Automação e Controle (LAC).

Ao professor Dr. José Roberto Castilho Piqueira, pelo incentivo e recomendação de ingresso no programa de doutorado.

Aos alunos e colegas professores do Programa de Educação Continuada em Engenharia da Escola Politécnica da USP (PECE-EPUSP), Faculdade de Tecnologia de São Paulo (Fatec-SP) e Centro Universitário da Fundação Santo André (FSA), que me incentivaram a seguir a carreira docente. Aos colegas do Itaú Unibanco, pelo incentivo à conclusão desta tese.

À memória do Eng. Salvador Arena, por seu ideal ao criar o Colégio Termomecanica, onde tive o prazer de estudar.

À Fundação de Apoio à Universidade de São Paulo (FUSP), pelo apoio financeiro para minha participação no Congresso Brasileiro de Automática em 2014.

Agradeço especialmente às pessoas queridas que fazem parte da minha vida, pois sem elas nada disso seria possível. À minha esposa e aos meus pais, pelo amor, carinho, incentivo e paciência incondicionais. A todos os meus amigos e familiares, pelo enorme incentivo. 


\section{Abstract}

This thesis deals with discrete-time Markov jump linear systems (MJLS) with Markov chain in a general Borel space $\mathcal{S}$. Several control issues have been addressed for this class of dynamic systems, including stochastic stability (SS), linear quadratic (LQ) optimal control synthesis, filter design and a separation principle. Necessary and sufficient conditions for SS have been derived. It was shown that SS is equivalent to the spectral radius of an operator being less than 1 or to the existence of a solution to a "Lyapunov-like" equation. Based on the SS concept, the finite- and infinite-horizon LQ optimal control problems were tackled. The solution to the finite- (infinite-)horizon LQ optimal control problem was derived from the associated control $\mathcal{S}$-coupled Riccati difference (algebraic) equations. By $\mathcal{S}$-coupled it is meant that the equations are coupled via an integral over a transition probability kernel having a density with respect to a $\sigma$-finite measure on the Borel space $\mathcal{S}$. The design of linear Markov jump filters was analyzed and a solution to the finite- (infinite-)horizon filtering problem was obtained based on the associated filtering $\mathcal{S}$-coupled Riccati difference (algebraic) equations. Conditions for the existence and uniqueness of a stabilizing positive semi-definite solution to the control and filtering $\mathcal{S}$ coupled algebraic Riccati equations have also been derived. Finally a separation principle for discrete-time MJLS with Markov chain in a general state space was obtained. It was shown that the optimal controller for a partial information optimal control problem separates the partial information control problem into two problems, one associated with a filtering problem and the other associated with an optimal control problem with complete information. It is expected that the results obtained in this thesis may motivate further research on discrete-time MJLS with Markov chain in a general state space.

Keywords: Linear Systems. Stochastic Control. Optimal Control. Markov Chains. Riccati Equations. 


\section{Resumo}

Esta tese trata de sistemas lineares com saltos markovianos (MJLS) a tempo discreto com cadeia de Markov em um espaço geral de Borel $\mathcal{S}$. Vários problemas de controle foram abordados para esta classe de sistemas dinâmicos, incluindo estabilidade estocástica (SS), síntese de controle ótimo linear quadrático (LQ), projeto de filtros e um princípio da separação. Condições necessárias e suficientes para a SS foram obtidas. Foi demonstrado que SS é equivalente ao raio espectral de um operador ser menor que 1 ou à existência de uma solução para uma equação de Lyapunov. Os problemas de controle ótimo a horizonte finito e infinito foram abordados com base no conceito de SS. A solução para o problema de controle ótimo LQ a horizonte finito (infinito) foi obtida a partir das associadas equações a diferenças (algébricas) de Riccati $\mathcal{S}$-acopladas de controle. Por $\mathcal{S}$-acopladas entende-se que as equações são acopladas por uma integral sobre o kernel estocástico com densidade de transição em relação a uma medida $\sigma$-finita no espaço de Borel $\mathcal{S}$. O projeto de filtros lineares markovianos foi analisado e uma solução para o problema da filtragem a horizonte finito (infinito) foi obtida com base nas associadas equações a diferenças (algébricas) de Riccati $\mathcal{S}$-acopladas de filtragem. Condições para a existência e unicidade de uma solução positiva semi-definida e estabilizável para as equações algébricas de Riccati $\mathcal{S}$-acopladas associadas aos problemas de controle e filtragem também foram obtidas. Por último, foi estabelecido um princípio da separação para MJLS a tempo discreto com cadeia de Markov em um espaço de estados geral. Foi demonstrado que o controlador ótimo para um problema de controle ótimo com informação parcial separa o problema de controle com informação parcial em dois problemas, um deles associado a um problema de filtragem e o outro associado a um problema de controle ótimo com informação completa. Esperase que os resultados obtidos nesta tese possam motivar futuras pesquisas sobre MJLS a tempo discreto com cadeia de Markov em um espaço de estados geral.

Palavras-chave: Sistemas lineares. Controle estocástico. Controle ótimo. Cadeias de Markov. Equações de Riccati. 


\section{List of Figures}

1 A concentrated solar power plant (Source: Costa et al. (2005c)) . . . . . . 103

2 Solution $X$ of the control $\mathcal{S}$-coupled algebraic Riccati equations for the solar thermal receiver . . . . . . . . . . . . . . . . . . . . . . 105

3 Optimal gains $K$ for the solar thermal receiver . . . . . . . . . . 106

4 A WTGS with horizontal-axis wind turbine (Source: Bianchi et al. (2007)) 107

5 State variables of the wind turbine generator system . . . . . . . . . . 113 


\section{List of Tables}

1 Parameters for the solar thermal receiver model . . . . . . . . . . . . . . . 104

2 Parameters for the wind turbine generator system . . . . . . . . . . . . . . 112 


\section{List of Abbreviations}

LQ Linear Quadratic

LQG Linear Quadratic Gaussian

MJLS Markov Jump Linear System

SS Stochastic Stability

WTGS Wind Turbine Generator System 


\section{List of Symbols}

\begin{tabular}{ll}
$\|\cdot\|$ & Any norm \\
$\|\cdot\|$ & Euclidean norm \\
$\|\cdot\|$ & Induced uniform operator norm \\
$\|\cdot\|_{1}$ & 1-norm \\
$\|\cdot\|_{1}$ & Induced 1-norm \\
$\|\cdot\|_{\text {sup }}$ & Essential supremum norm \\
$\|\cdot\|_{\text {sup }}$ & Induced essential supremum norm \\
$\|\cdot\|_{V}$ & The norm defined as $\|x\|_{V}^{2}=x^{*} V x$, for $x \in \mathbb{C}^{n}$ and $V>0, V \in \mathbb{B}_{\left(\mathbb{C}^{n}\right)}$ \\
$\ll$ & Absolutely continuous \\
1. & The indicator function \\
$\delta$. & The Dirac measure \\
$\mu$ & $\sigma$-finite measure on $\mathcal{S}$ \\
$\widehat{\mu}_{\mathcal{S}}$ & The initial probability measure on $\mathcal{S}$ for $\theta_{0}$ \\
$\sigma(\cdot)$ & The spectrum of a bounded linear operator \\
$\theta(k)$ & Markov state at time $k$ \\
$\theta_{0}$ & $\theta_{0}=\theta(0)$ \\
$\mathcal{B}(\mathcal{X})$ & Borel $\sigma$-algebra of $\mathcal{X}$ \\
$g(\cdot, \cdot)$ & probability density of $\mathcal{G}(\cdot, \cdot)$ with respect to $\mu$ \\
$\mathcal{G}(\cdot, \cdot)$ & $\mu$-continuous transition probability function \\
$I$ & The identity operator \\
$I$ & The $n \times n$ identity matrix, for some positive integer $n$ \\
$\mathcal{P}$ & Probability \\
$r_{\sigma}(\cdot)$ & The family of all stochastic kernels on $\mathcal{X}$ given $\mathcal{Y}$ \\
$\operatorname{tr}(\cdot)$ & The trace operator \\
$u(k)$ & Control variable at time $k$ \\
\hline &
\end{tabular}


$x(k) \quad$ System state at time $k$

$x_{0} \quad x_{0}=x(0)$

$y(k) \quad$ Measurable variable at time $k$

$\mathbb{B}(\mathbb{X}, \mathbb{Y}) \quad$ The Banach space of all bounded linear operators of $\mathbb{X}$ into $\mathbb{Y}$

$\mathbb{B}(\mathbb{X}) \quad \mathbb{B}(\mathbb{X}):=\mathbb{B}(\mathbb{X}, \mathbb{X})$

$\mathbb{C}^{n} \quad$ The $n$-dimensional complex Euclidean space

$\mathbb{H}_{1}^{n, m} \quad$ Banach space

$\mathbb{H}_{\text {sup }}^{n, m} \quad$ Banach space

$\mathbb{N} \quad$ The set of nonnegative integers

$\mathbb{N}_{1} \quad$ The set of positive integers

$\mathbb{R}^{+} \quad$ The interval $[0, \infty)$

$\mathbb{R}^{n} \quad$ The $n$-dimensional real Euclidean space

$\mathcal{S} \quad$ Borel space

T The discrete-time set

$\mathbb{T} \quad$ The set of nonnegative integers less or equal to $\tau-1$, for a fixed positive integer $\tau>1$

$\mathbb{T}_{1} \quad$ The set of nonnegative integers greater than 1 and less or equal to $\tau-1$, for a fixed positive integer $\tau>1$

$\mathcal{X} \quad$ Borel space

$\mathbb{X} \quad$ Complex Banach space

$\mathcal{Y} \quad$ Borel space

$\mathbb{Y} \quad$ Complex Banach space 


\section{Contents}

1 Introduction $\quad 13$

1.1 Motivation . . . . . . . . . . . . . . . . . . 13

1.2 Thesis Contributions . . . . . . . . . . . . . . . . . 14

1.3 Organization of the Thesis . . . . . . . . . . . . . . 16

2 Discrete-Time Markov Jump Linear Systems 18

2.1 Stability . . . . . . . . . . . . . . . . . . . 18

2.2 Optimal Control . . . . . . . . . . . . . . . . . 20

3 Discrete-Time MJLS with Markov Chain in a General State Space 24

3.1 Notation and Auxiliary Results . . . . . . . . . . . . . . . . 24

3.2 Probabilistic Space . . . . . . . . . . . . . . . . . . . . . . . 29

3.3 Discrete-Time MJLS with Markov Chain in a General State Space . . . . . 31

4 Stochastic Stability 33

4.1 Stochastic Stability Definition . . . . . . . . . . . . . . 33

4.2 Auxiliary Results . . . . . . . . . . . . . . . . . . . 35

4.3 Conditions for Stochastic Stability . . . . . . . . . . . . . . . . 38

5 LQ Optimal Control $\quad 46$

5.1 Finite-Horizon LQ Optimal Control . . . . . . . . . . . . . . . 46

$5.1 .1 \quad$ Problem Statement . . . . . . . . . . . . . . . . 47

5.1 .2 The Optimal Control Law . . . . . . . . . . . . . . . . . . 47

5.2 Infinite-Horizon LQ Optimal Control . . . . . . . . . . . . . . . . . . 49

5.2.1 Problem Statement . . . . . . . . . . . . . . . . . . . . 49

5.2 .2 The Optimal Control Law . . . . . . . . . . . . . . . . . . 50

$\begin{array}{llr}6 & \text { Filtering } & 53\end{array}$

6.1 Finite-Horizon Filtering $\ldots \ldots \ldots \ldots \ldots$ 
6.1.1 Problem Statement . . . . . . . . . . . . . . . . . 54

6.1.2 Finite-Horizon Optimal Filter . . . . . . . . . . . . . . . 58

6.2 Infinite-Horizon Filtering . . . . . . . . . . . . . . 70

6.2.1 Problem Statement . . . . . . . . . . . . . . 70

6.2.2 Infinite-Horizon Optimal Filter . . . . . . . . . . . . . 73

7 Optimal Control with Partial Information $\quad 77$

7.1 Finite-Horizon LQ Optimal Control with Partial Information . . . . . . . . 78

7.1.1 Problem Statement . . . . . . . . . . . . . . . 78

7.1 .2 Complete Information Case . . . . . . . . . . . . . . 80

7.1.3 A Separation Principle . . . . . . . . . . . . . 87

7.2 Infinite-Horizon $H_{2}$ Optimal Control f . . . . . . . . . . . . . 88

7.2.1 $\mathrm{H}_{2}$ Optimal Control Problem . . . . . . . . . . . . 88

7.2.2 Alternative Definition for the $H_{2}$ Optimal Control Problem . . . . . 92

7.2.3 A Separation Principle . . . . . . . . . . . . . . . 95

8 Applications $\quad 102$

8.1 LQ Optimal Control for a Solar Thermal Receiver . . . . . . . . . . . . . . 102

8.2 LQ Optimal Control with Partial Information for a Wind Turbine Generator System . . . . . . . . . . . . . . . . . . 106

9 Conclusion $\quad 114$

9.1 Directions for Future Research . . . . . . . . . . . . . . . . . 114

9.2 Final Remarks and Summary of Contributions . . . . . . . . . . . 116

$\begin{array}{ll}\text { Bibliography } & 117\end{array}$

A $\mathcal{S}$-Coupled Algebraic Riccati Equations $\quad 124$

A.1 Control $\mathcal{S}$-Coupled Algebraic Riccati Equations . . . . . . . . . . . . . 124

A.1.1 Control $\mathcal{S}$-Coupled Algebraic Riccati Equations . . . . . . . . . . . 124

A.1.2 Auxiliary Results . . . . . . . . . . . . . . . . . . 125

A.1.3 Stationary Case . . . . . . . . . . . . . . . 126

A.2 Filtering $\mathcal{S}$-Coupled Algebraic Riccati Equations . . . . . . . . . . . . 133

A.2.1 Filtering $\mathcal{S}$-Coupled Algebraic Riccati Equations . . . . . . . . . . . 133

A.2.2 Auxiliary Results . . . . . . . . . . . . . . . . 137

A.2.3 Stationary Case . . . . . . . . . . . . . . . . . . . . 138 


\section{Chapter 1}

\section{Introduction}

\subsection{Motivation}

Linear systems with the dynamics subject to abrupt changes have been the object of increasing research over the last years, especially the case in which the abrupt changes are modeled by a jump parameter that follows a Markov chain. This class of models is referred to in the international literature as Markov jump linear systems (MJLS) and there is already an extensive body of results on this subject. One can refer to the works of Zhang et al. (2016), Costa et al. (2013), Dragan et al. (2010), Boukas (2006), Costa et al. (2005c), Czornik (2003) and Mariton (1990) as a sample of books on this topic.

The great interest in this class of systems is due to the breadth of application of MJLS to model systems and processes in many fields. Without attempting to be exhaustive, applications of these models can be found in robotics (see Siqueira and Terra, 2009), tracking and estimation (see Dufour and Bertrand, 1996; Evans and Evans, 1999; Sworder and Hutchins, 1989; Sworder et al., 1993), mathematical finance (see Blake and Zampolli, 2011; Costa and Araujo, 2008; Zampolli, 2006), communication networks (see Gonçalves et al., 2010; Hu and Yan, 2007; Seiler and Sengupta, 2005), fault tolerant control (see Aberkane et al., 2008; Mahmoud et al., 2003; Mariton, 1989; Srichander and Walker, 1993), and flight systems (see Gray et al., 2000; Mariton and Bertrand, 1987).

Most of the MJLS literature deals with control problems for the case in which the state space of the Markov chain is finite or countably infinite. Considering these cases, some fundamental control issues such as stability and stabilization, control synthesis by different methodologies, estimation and filtering, have been extensively studied and many results are available in the literature. Only more recently the case in which the Markov chain 
takes values in a general (non-countable) state space started to be analyzed. Despite some issues have already been tackled for MJLS with Markov chain in a general state space, many important control topics regarding stability analysis, optimal control synthesis and filter design have not been studied. It is expected that several real world applications might benefit by a further analysis of the general state space case, for instance, systems subject to abrupt changes due to environmental disturbances (see Chapter 8), networked control systems where network-induced communication imperfections vary in a stochastic way (see Kordonis and Papavassilopoulos, 2014), among others. This scenario motivates the investigation of discrete-time MJLS, considering the case in which the Markov chain takes values in a Borel space $\mathcal{S}$. The focus on discrete-time models is justified by its broad range of application. Many phenomena are naturally modeled in discrete-time, including economic systems, management and production systems, storage systems, population growth, epidemic spread, and systems that employ digital technology, like computers and telecommunications networks, just to name a few. Furthermore, many continuous-time systems can only be treated or simulated via a discrete-time approximation.

\subsection{Thesis Contributions}

This doctoral thesis addresses several control issues for discrete-time MJLS with Markov chain in a general state space. The main contributions of this thesis are summarized as follows:

- Stability Analysis: Necessary and sufficient conditions for the stochastic stability (SS) of discrete-time MJLS with Markov chain in a Borel space $\mathcal{S}$ are derived in Chapter 4. It is shown that SS is equivalent to the spectral radius of an operator being less than 1 or to the existence of a solution to a "Lyapunov-like" equation. These results have been published in the journal IEEE Transactions on Automatic Control as a technical note (see Costa and Figueiredo, 2014);

- LQ Optimal Control: The linear quadratic (LQ) optimal control considering complete information, for the finite- and infinite-horizon optimal control problems, is addressed in Chapter 5. It is assumed that the controller has access to the system's state variable as well as to the jump parameter (the operation mode of the system). These control problems are similar to the classical LQ regulator problems considered for discrete-time linear systems (see Davis and Vinter, 1985, Section 6.1), but adapted for MJLS with Markov chain in a Borel space $\mathcal{S}$. The solution to the 
finite-horizon LQ optimal control problem is derived in Section 5.1 from $\mathcal{S}$-coupled difference Riccati equations (coupled via an integral over a transition probability kernel having a density with respect to a $\sigma$-finite measure on the Borel space $\mathcal{S}$ ), named control $\mathcal{S}$-coupled difference Riccati equations. In Section 5.2 the solution to the infinite-horizon LQ optimal control problem is obtained from the stabilizing positive semi-definite solution of the control $\mathcal{S}$-coupled algebraic Riccati equations. Sufficient conditions, based on the concepts of (stochastic) stabilizability and detectability, for the existence and uniqueness of a stabilizing positive semi-definite solution to the control $\mathcal{S}$-coupled algebraic Riccati equations are obtained in Appendix A.1. These results have been published in the journal IEEE Transactions on Automatic Control as a technical note (see Costa and Figueiredo, 2015);

- Filter design: In the filtering problems tackled in this thesis, the goal is to design a minimum mean square linear Markov jump filter. It is assumed that a measurable output and the jump parameter are known at each discrete-time instant, and only the family of linear Markov jump filters is considered (that is, filters that only depend, at each discrete-time instant, on the present values of the Markov parameter and measurable output), since otherwise the optimal linear mean square filter would be obtained from a sample path Kalman filter (see Davis and Vinter, 1985, Chapter $3)$. Both the finite- and infinite-horizon cases are studied. These problems are similar to the filtering problems considered for MJLS with Markov Chain in a finite space (see Costa et al., 2005c, Chapter 5), but adapted for the general state space case. The solution to the finite-horizon filtering problem is presented in Section 6.1 and it is associated to $\mathcal{S}$-coupled difference Riccati equations, named filtering $\mathcal{S}$-coupled difference Riccati equations. This result have been published in the journal Automatica as part of a regular paper (see Costa and Figueiredo, 2016). In Section 6.2 the infinite-horizon filtering-problem is tackled and its solution is obtained in terms of the stabilizing positive semi-definite solution of the filtering $\mathcal{S}$-coupled algebraic Riccati equations. Conditions for the existence and uniqueness of a stabilizing positive semi-definite solution to the filtering $\mathcal{S}$-coupled algebraic Riccati equations are derived in Appendix A.2 in terms of the concepts of (stochastic) stabilizability and detectability. These results involving the infinite-horizon filtering-problem and the filtering $\mathcal{S}$-coupled algebraic Riccati equations have been submitted to publication and are currently under review;

- Separation Principle: The optimal control considering the partial information 
case is analyzed in Chapter 7. It is assumed that the controller has access only to a measurable output variable and to the jump parameter, and, differently from the complete information case, the goal is to design a Markov jump controller. In Section 7.1 the finite-horizon case is analyzed and it is shown that an optimal controller can be obtained from two $\mathcal{S}$-coupled difference Riccati equations, one associated with a filtering problem and the other one with a control problem in which the state variable is fully available to the controller. This result have been published as the final part of the paper by Costa and Figueiredo (2016). The infinite-horizon LQ optimal control is tackled from a $H_{2}$ optimal control perspective in Section 7.2. It is shown that the $H_{2}$-norm can be written as the sum of two $H_{2}$-norms, such that one of them does not depend on the control and the other is obtained from the optimal filter for an infinite-horizon filtering problem. A paper presenting this result is currently in preparation. Notice that similarly to the discrete-time linear systems case, the results obtained in Chapter 7 show that the optimal controller separates a partial information optimal control problem into two problems, one associated with a filtering problem and the other associated with an optimal control problem with complete information. These results can be seen as a separation principle for discrete-time MJLS with Markov chain in a general state space.

It is worth mentioning that the approach adopted in this thesis is theoretically oriented, although two illustrative numerical examples have been presented in Chapter 8. It is expected that the results obtained in this thesis may motivate further research on discretetime MJLS with Markov chain in a general state space.

\subsection{Organization of the Thesis}

The rest of this thesis is organized as follows. In Chapter 2 a literature review focusing on discrete-time MJLS is presented. Chapter 3 formally introduces the class of MJLS that is studied in this thesis, in addition to presenting some background material. Chapter 4 deals with the stochastic stability for MJLS with Markov chain in a general state space. Chapter 5 treats the LQ optimal control with complete information. The design of minimum mean square linear Markov jump filters is considered in Chapter 6. Chapter 7 addresses the LQ optimal control with partial information. In this case the system's state variable is not directly accessible to the controller, but, instead, it is assumed that only a measurable output and the jump parameter are available. Chapter 8 presents 
applications from some of the theoretical results obtained in this thesis. Directions for future research and a summary of contributions offered by this thesis are presented in Chapter 9. Finally the control and filtering $\mathcal{S}$-coupled algebraic Riccati equations are considered in the Appendix. 


\section{Chapter 2}

\section{Discrete-Time Markov Jump Linear Systems}

The literature on Markov jump linear systems is by now quite extensive. This chapter presents a discussion addressing the relationship between the results obtained in this thesis and those available in the discrete-time MJLS literature. Focused on works relevant to the research presented in this thesis, Section 2.1 provides a review of works on stability issues and Section 2.2 presents works dealing with optimal control synthesis and filter design. Refer to the books by Costa et al. (2005c) and Mariton (1990) for a more comprehensive and historical perspective regarding MJLS.

\subsection{Stability}

Stability is a main concern of feedback loop design and is usually the first requirement to be considered in a control system. If a system is not stable, a suitable controller has to be designed in order to stabilize it and impose the desired behavior on the closed loop system, therefore stability plays a key issue in both the analysis and the design of control systems. Several concepts of stability for jump linear systems can be found in the literature, such as almost sure (asymptotic) stability, mean square stability ( $\delta$-moment stability for $\delta=2$ ), exponential mean square stability, and stochastic stability.

Costa and Fragoso (1993) presented necessary and sufficient conditions for mean square stability of discrete-time MJLS with Markov chain in a finite state space. These conditions are given in terms of spectral radius of an augmented matrix being less than 1 or in terms of the existence of a positive semi-definite solution to a "Lyapunov-like" equation. These authors also showed that mean square stability implies almost sure stability. 
Czornik (2003, Chapter 3) presented a detailed review of papers dealing with stability for discrete-time MJLS with Markov chain in a finite state space. It was shown that mean square stability and stochastic stability are equivalent for this class of dynamic systems, and, furthermore, $\delta$-moment stability implies almost sure stability (see Czornik, 2003, Theorems 37 and 38).

Sample path and moments stability are the most important stability concepts for applications. In real world control systems one is interested in the stability of individual sample paths of the system, and thus almost sure stability is much more desirable than any moment stability. Despite of that, concepts like mean square and stochastic stability are also of great concern. The concept of stochastic stability implies a finite cost for the infinite-horizon linear quadratic (LQ) optimal control problem (Feng et al., 1992), and, as already mentioned, mean square and stochastic stability provide sufficient conditions for almost sure stability.

Regarding the mean square stability theory for discrete-time MJLS, one can refer to the papers by Ling and Deng (2012), Fang and Loparo (2002), Costa and Fragoso (1993) and Ji et al. (1991), as a sample of works on this subject, which has by now a fairly complete body of results. Regarding other issues such as almost sure stability, robust stability, stabilizability and detectability, the reader is referred, for instance, to the papers by Costa et al. (2006), Costa et al. (2005a), Bolzern et al. (2004), Costa and do Val (2002), Fang (1997) and Fang et al. (1994) (see the books by Costa et al. (2005c) and Czornik (2003) for further references).

In all these works the state space of the Markov chain was assumed to take values in a finite state space. But, as pointed out by Costa and Fragoso (1995), there are some important differences between the finite and infinite countable cases. For instance, Costa and Fragoso (1995, Remark 6) showed that for the case in which the state space of the Markov chain is countably infinite, mean square and stochastic stability are no longer equivalent. Due to that, the case in which the Markov chain is countably infinite has received some attention (see Costa et al., 2005b; Ungureanu, 2009; Ungureanu et al., 2013).

Only more recently the general state space case, in which the Markov chain takes values in a general state space, started to be analyzed. Regarding some stability results it can be mentioned the papers by Li et al. (2012) and Kordonis and Papavassilopoulos (2014), in which the exponential almost sure stability and mean square stability were considered. Assuming that the Markov chain is a positive Harris chain, Li et al. (2012) showed in Theorem 4.3 that the uniform exponentially almost sure stability is equivalent 
to a contractivity condition being satisfied.

Differently from the paper by Li et al. (2012), this thesis deals with the stochastic stability concept. No positive Harris assumptions are required, and necessary and sufficient conditions for stochastic stability are derived. It is shown in Chapter 4 that stochastic stability is equivalent to the spectral radius of a bounded linear operator in a Banach space being less than 1 or to the existence of a solution to a "Lyapunov-like" equation.

\subsection{Optimal Control}

The problem of optimal control synthesis for discrete-time MJLS have been largely studied. Similarly to the stability case, most of the literature dealing with control problems for discrete-time MJLS considers that the Markov chain takes value in a finite set. Some of the published works on this issue have been done by Dragan et al. (2010), Czornik (2003) and Ji et al. (1991). For a systematic analysis of the finite and infinite-horizon linear quadratic (LQ) optimal control problems refer to Costa et al. (2005c, Chapter 4). The results obtained for these problems have many similarities to those obtained for the LQ regulator problem for discrete-time linear systems (see Davis and Vinter, 1985, Section 6.1). The optimal controller associated with the finite- (infinite-)horizon problem has a state feedback form and the control gains are defined from a set of quadratic matrix equations, named coupled Riccati difference (algebraic) equations. Conditions for existence and uniqueness of a positive semi-definite solution to the associated control coupled algebraic Riccati equations (CARE) are given in terms of the concepts of (mean square) stabilizability and detectability (see Costa et al., 2005c, Appendix A).

Costa and Fragoso (1995) have tackled the finite- (infinite-)horizon LQ optimal control problems for discrete-time MJLS with Markov chain in a countably infinite state space. The obtained solution of the infinite-horizon LQ optimal control problem is associated to a countably infinite set of coupled algebraic Riccati equations. These authors have also derived conditions for the existence and uniqueness of a positive semi-definite solution to the infinite coupled algebraic Riccati equations (ICARE) via the concepts of (stochastic) stabilizability and detectability. Ungureanu et al. (2013), Ungureanu (2009) and Costa et al. (2005b) have also dealt with the LQ optimal control of discrete-time MJLS with Markov chain in an infinite countable state space.

Kordonis and Papavassilopoulos (2014) have characterized the finite and infinitehorizon LQ optimal control problems for MJLS with Markov chain in a general state space in terms of appropriate Riccati integral equations. Kordonis and Papavassilopoulos 
(2014) obtained that if there exists a police that yields a finite cost for the infinite-horizon LQ optimal control problem then the optimal cost has a quadratic form, written in terms of a solution to a Riccati integral equation, and the optimal control law is in a state feedback form, and, conversely, if there exists a solution to the Riccati integral equation and the feedback control law derived from this solution yields a mean square exponentially stable closed loop system, then this solution is optimal for the infinite-horizon LQ optimal control problem.

The finite-horizon LQ optimal control problem for discrete-time MJLS with Markov chain in a Borel space $\mathcal{S}$ is tackled in Section 5.1 of this thesis. An optimal controller is obtained from $\mathcal{S}$-coupled Riccati difference equations. In Section 5.2 and Appendix A.1 the infinite-horizon LQ optimal control problem and the associated $\mathcal{S}$-coupled algebraic Riccati equations are analyzed. The main result presented in Appendix A.1 states that, under some conditions based on the concepts of (stochastic) stabilizability and detectability, there exists a unique (in the $\mu$-almost everywhere sense) stabilizing positive-semidefinite solution for the control $\mathcal{S}$-coupled algebraic Riccati equations and, as shown in Section 5.2 , it provides a solution to the infinite-horizon LQ optimal control problem. Notice that these results, based on the concepts of (stochastic) stabilizability and detectability, are substantially different from the results obtained by Kordonis and Papavassilopoulos (2014).

One can refer to the works by Costa et al. (2005c), Costa (1995) and Dragan et al. (2010) regarding the finite and infinite-horizon filtering problems for discrete-time MJLS with Markov chain taking values in a finite set. In the filtering problems tackled by these authors it was assumed that a measurable output and the jump parameter were known at each discrete-time instant. Recall that the optimal $x$-state estimator for this case is a Kalman filter for a time varying system, and therefore the gains are sample path dependent. To avoid this sample path dependence the optimal estimator was taken from a class of filters, called Markov jump filters, that only depend, at each discretetime instant, on the current measurable output and mode of operation (jump parameter). Costa (1995) obtained, based on the concepts of (mean square) stabilizability and detectability, conditions for the existence and uniqueness of a positive semi-definite solution to a set of coupled algebraic Riccati equations (filtering CARE), which is related to the infinite-horizon filtering problem. The optimal filter for the finite- (infinite-)horizon filtering problem was derived based on the filtering coupled Riccati difference (algebraic) equations (see Costa et al., 2005c, Chapter 5).

Chapter 6 and Appendix A.2 of this thesis present results related to the filtering 
problems for MJLS with Markov chain in a general state space case. The solution to the finite- (infinite-)horizon filtering problem is obtained from $\mathcal{S}$-coupled Riccati difference (algebraic) equations. Conditions for the existence and uniqueness of a stabilizing positive semi-definite solution to the associated filtering $\mathcal{S}$-coupled algebraic Riccati equations are derived in Appendix A.2 in terms of the concepts of (stochastic) stabilizability and detectability. These results for the general state space case can be seen as an extension of the results obtained by Costa (1995) and Costa et al. (2005c), which considered the finite state space case.

The partial information control and filtering problems can be associated to the cases in which the state variable is not observable, the Markov chain parameter is not observable, or yet when both variables are not available. Regarding MJLS, it can be mentioned the papers by Fragoso and Costa (2010), Costa and Fragoso (2007), Costa and Tuesta (2003), de Farias et al. (2000), and Dufour and Elliott (1997), as a sample of works dealing with the case with noisy observation of the state variable but perfect information of the jump parameter. For the case in which the partial observation is referred to the jump parameter it can be mentioned, for instance, the papers by Costa et al. (2015), Everdij and Blom (1996), Caines and Zhang (1995), Fragoso (1990) and Fragoso (1988). The case in which both variables are not available was considered, for instance, by Costa and Guerra (2002a), Costa and Guerra (2002b), Dufour and Elliott (1997), Bar-Shalom and Li (1993), Tugnait (1982), and Ackerson and Fu (1970), among others.

Tracing a parallel to the linear quadratic Gaussian (LQG) theory for stochastic linear systems (see Davis and Vinter, 1985, Section 6.3), Costa and Tuesta (2003, 2004) considered the finite and infinite-horizon quadratic optimal control problems of discrete-time MJLS with finite state Markov chain, in which only a measurable output and the jump parameters of the system are available to the controller. Recall again that the optimal state estimator for this case is a Kalman filter for a time varying system. To avoid the sample path dependence, Costa and Tuesta (2003, 2004) considered the quadratic cost among the class of Markov jump controllers, that is, controllers that only depend, at each discrete-time instant, on the current measurable output and mode of operation. Similarly as in the case in which there is only one mode of operation, it was shown by Costa and Tuesta $(2003,2004)$ that an explicit solution to the finite- (infinite)-horizon optimal control problem can be derived from two coupled Riccati difference (algebraic) equations, one of them associated to the optimal controller when the state variables are available, and the other one associated to a filtering problem. This property is usually known as the separation principle. In Chapter 7 of this thesis similar results to those 
obtained by Costa and Tuesta $(2003,2004)$ are derived for MJLS with Markov chain in a general state space, including a separation principle for this class of dynamic systems. 


\section{Chapter 3}

\section{Discrete-Time MJLS with Markov Chain in a General State Space}

This chapter is devoted to present the class of dynamic systems that is studied throughout this thesis, namely the discrete-time Markov jump linear systems (MJLS) with Markov chain in a general state space. With this goal in mind, in Section 3.1 some notational conventions and useful spaces are introduced. Beyond that, some basic results are also presented. In Section 3.2 the probabilistic space considered in this thesis is introduced. Finally in Section 3.3 the model that describes a discrete-time MJLS with Markov chain in a general state space is formally presented.

\subsection{Notation and Auxiliary Results}

The notation and the definitions that follow are pretty standard in functional analysis literature (see Kreyszig, 1978; Naylor and Sell, 1982) and are employed throughout this thesis.

Suppose that $\mathbb{X}$ and $\mathbb{Y}$ are complex Banach spaces. The Banach space of all bounded linear operators of $\mathbb{X}$ into $\mathbb{Y}$, with the (induced uniform) operator norm represented by $\|\cdot\|$, will be denoted by $\mathbb{B}(\mathbb{X}, \mathbb{Y})$. For simplicity it is defined $\mathbb{B}(\mathbb{X}):=\mathbb{B}(\mathbb{X}, \mathbb{X})$. Recall that, for $\mathcal{T} \in \mathbb{B}(\mathbb{X})$, by induced uniform norm it is meant that $\|\mathcal{T}\|=\sup _{x \neq 0} \frac{\|\mathcal{T}(x)\|}{\|x\|}$, $x \in \mathbb{X}$. In the present thesis the words function, map, mapping, transformation, and correspondence must be taken as synonyms. The term operator is used for mappings between vector spaces and, in particular, between normed spaces (Kreyszig, 1978, p. 82).

For $\mathcal{T} \in \mathbb{B}(\mathbb{X})$ it is denoted by $\sigma(\mathcal{T})$ the spectrum of the operator $\mathcal{T}$ and by $r_{\sigma}(\mathcal{T})=$ $\max \{|\lambda| ; \lambda \in \sigma(\mathcal{T})\}$ its spectral radius. Recall that the spectrum of a bounded linear 
operator $\mathcal{T}$ on a complex Banach space is compact and lies in the disk given by $|\lambda| \leq\|\mathcal{T}\|$ (Kreyszig, 1978, Theorem 7.3-4).

The $n$-dimensional complex (real) Euclidean space is denoted by $\mathbb{C}^{n}\left(\mathbb{R}^{n}\right.$, respectively) and the normed bounded linear space of all $m \times n$ complex matrices by $\mathbb{B}\left(\mathbb{C}^{n}, \mathbb{C}^{m}\right)$, with $\mathbb{B}\left(\mathbb{C}^{n}\right):=\mathbb{B}\left(\mathbb{C}^{n}, \mathbb{C}^{n}\right)$ and $\mathbb{B}\left(\mathbb{C}^{n}\right)^{+}:=\left\{L \in \mathbb{B}\left(\mathbb{C}^{n}\right) ; L=L^{*} \geq 0\right\}$. In this case, the superscripts,,$-^{\prime}$, and * denote complex conjugate, transpose and conjugate transpose, respectively. The eigenvalues of a matrix $P \in \mathbb{B}\left(\mathbb{C}^{n}\right)$ are denoted by $\lambda_{i}(P), i=1, \ldots, n$. Either the standard Euclidean norm in $\mathbb{C}^{n}$ or the (induced uniform) operator norm in $\mathbb{B}\left(\mathbb{C}^{n}\right)$ are represented by $\|\cdot\|$. For $V>0, V \in \mathbb{B}\left(\mathbb{C}^{n}\right)$, the norm $\|\cdot\|_{V}$ in $\mathbb{C}^{n}$ is defined as $\|x\|_{V}^{2}=x^{*} V x$.

The following result is used in the next chapters. Its proof can be found in (Costa et al., 2005c, p. 18).

Remark 3.1.1. For any $W \in \mathbb{B}\left(\mathbb{C}^{n}\right)$ there exist $W^{j}, j=1,2,3,4$, such that $W^{j} \geq 0$, $\left\|W^{j}\right\| \leq\|W\|$ for $j=1,2,3,4$, and $W=\left(W^{1}-W^{2}\right)+\sqrt{-1}\left(W^{3}-W^{4}\right)$.

The trace operator is denoted by $\operatorname{tr}(\cdot)$. Recall that the trace is a linear functional $\operatorname{tr}(\cdot): \mathbb{B}\left(\mathbb{C}^{n}\right) \rightarrow \mathbb{C}$, satisfying the properties

$$
\operatorname{tr}(K L)=\operatorname{tr}(L K)
$$

and for any $M, P \in \mathbb{B}\left(\mathbb{C}^{n}\right)$ with $M \geq 0, P>0$,

$$
\left(\min _{i=1, \ldots, n} \lambda_{i}(P)\right) \operatorname{tr}(M) \leq \operatorname{tr}(M P) \leq\left(\max _{i=1, \ldots, n} \lambda_{i}(P)\right) \operatorname{tr}(M)
$$

The operator that creates a column vector from a matrix $A$ by stacking the column vectors of $A$ below one another is denoted by $\operatorname{vec}(\cdot)$. For $M, P \in \mathbb{B}\left(\mathbb{C}^{n}\right)$,

$$
\operatorname{tr}\left(M^{*} P\right)=\operatorname{vec}(M)^{*} \operatorname{vec}(P)
$$

and therefore

$$
\left|\operatorname{tr}\left(M^{*} P\right)\right|=\left|\operatorname{vec}(M)^{*} \operatorname{vec}(P)\right| \leq\|\operatorname{vec}(M)\|\|\operatorname{vec}(P)\|=\sqrt{\operatorname{tr}\left(M^{*} M\right) \operatorname{tr}\left(P^{*} P\right)} .
$$

Moreover, for any $A \in \mathbb{B}\left(\mathbb{C}^{n}\right),\|A\|^{2}=\max _{i=1, \ldots, n} \lambda_{i}\left(A^{*} A\right)$ (Callier and Desoer, 1991, p. 443), and thus

$$
\|A\|^{2}=\max _{i=1, \ldots, n} \lambda_{i}\left(A^{*} A\right) \leq \operatorname{tr}\left(A^{*} A\right)=\sum_{i=1}^{n} \lambda_{i}\left(A^{*} A\right) \leq n\|A\|^{2} .
$$


A Borel subset of a complete and separable metric space is called a Borel space (see Hernandez-Lerma and Lasserre, 2012, p. 169). For a Borel space $\mathcal{X}$ its Borel $\sigma$-algebra is denoted by $\mathcal{B}(\mathcal{X})$.

Remark 3.1.2. Throughout the present thesis it is assumed that $\mathcal{S}$ is a fixed Borel space and $\mu$ is a fixed $\sigma$-finite measure on $\mathcal{S}$.

For $n, m$, positive integers, set $\mathbb{L}_{1}^{n m}$ as the Banach space of measurable functions $f(\cdot): \mathcal{S} \rightarrow \mathbb{C}^{n m}$ such that

$$
\|f\|_{1}=\int_{\mathcal{S}}\|f(t)\| \mu(d t)<\infty
$$

and $\mathbb{L}_{\text {sup }}^{n m}$ as the Banach space of measurable functions $f(\cdot): \mathcal{S} \rightarrow \mathbb{C}^{n m}$ such that

$$
\|f\|_{\text {sup }}=\operatorname{ess-\operatorname {sup}}\{\|f(t)\| ; t \in \mathcal{S}\}=\inf \{b ;\|f(t)\| \leq b \mu \text {-almost everywhere in } \mathcal{S}\}<\infty
$$

(Naylor and Sell, 1982, p. 220). When referring to either sets or functions, "measurable" means "Borel-measurable".

Assume that $P \in \mathbb{H}_{1}^{n, m}$ if $P(\cdot): \mathcal{S} \rightarrow \mathbb{B}\left(\mathbb{C}^{n}, \mathbb{C}^{m}\right)$ is measurable and

$$
\|P\|_{1}:=\int_{\mathcal{S}}\|P(t)\| \mu(d t)<\infty
$$

and, similarly, that $P \in \mathbb{H}_{\text {sup }}^{n, m}$ if $P(\cdot): \mathcal{S} \rightarrow \mathbb{B}\left(\mathbb{C}^{n}, \mathbb{C}^{m}\right)$ is measurable and

$$
\|P\|_{\text {sup }}:=\operatorname{ess}-\sup \{\|P(t)\| ; t \in \mathcal{S}\}<\infty
$$

For notational simplicity it is set $\mathbb{H}_{1}^{n}:=\mathbb{H}_{1}^{n, n}$, and $\mathbb{H}_{\text {sup }}^{n}:=\mathbb{H}_{\text {sup }}^{n, n}$. Also, it is denoted $\widetilde{\mathbb{H}}_{1}^{n, m}=\left\{L \in \mathbb{H}_{1}^{n, m} ; L(t)\right.$ is real, $\left.\forall t \in \mathcal{S}\right\}$. Similar notation is used for $\widetilde{\mathbb{H}}_{1}^{n}, \widetilde{\mathbb{H}}_{\text {sup }}^{n, m}, \widetilde{\mathbb{H}}_{\text {sup }}^{n}$, etc.

For any $P \in \mathbb{H}_{1}^{n, m}$ and setting $f(t)=\operatorname{vec}(P(t)), t \in \mathcal{S}$, it follows that $f \in \mathbb{L}_{1}^{n m}$ since from (3.1) and (3.2),

$$
\|f(t)\|^{2}=\operatorname{tr}\left(P(t)^{*} P(t)\right) \leq n\|P(t)\|^{2} .
$$

Similarly for any $f \in \mathbb{L}_{1}^{n m}$ and setting $P(t)=\operatorname{vec}^{-1}(f(t)), t \in \mathcal{S}$, it follows that $P \in \mathbb{H}_{1}^{n, m}$ since from (3.1) and (3.2),

$$
\|P(t)\|^{2} \leq \operatorname{tr}\left(P(t)^{*} P(t)\right)=\|f(t)\|^{2} .
$$

From this, one can conclude that $\left(\mathbb{L}_{1}^{n m},\|\cdot\|_{1}\right)$ and $\left(\mathbb{H}_{1}^{n, m},\|\cdot\|_{1}\right)$ are uniformly homeomorphic, and thus $\left(\mathbb{H}_{1}^{n, m},\|\cdot\|_{1}\right)$ is a Banach space. Similar reasoning shows that $\left(\mathbb{L}_{\text {sup }}^{n m},\|\cdot\|_{\text {sup }}\right)$ 
and $\left(\mathbb{H}_{\text {sup }}^{n, m},\|\cdot\|_{\text {sup }}\right)$ are uniformly homeomorphic and, thus, $\left(\mathbb{H}_{\text {sup }}^{n, m},\|\cdot\|_{\text {sup }}\right)$ is also a Banach space. It is worth pointing out that in the next chapters many operators are defined on the Banach spaces $\mathbb{H}_{1}^{n, m}$ and $\mathbb{H}_{\text {sup }}^{n, m}$ presented above. Also, the dynamic systems that are studied in this thesis have their parameters defined on the spaces $\mathbb{H}_{\text {sup }}^{n, m}$ and $\widetilde{\mathbb{H}}_{\text {sup }}^{n, m}$.

Since $\mathbb{L}_{\text {sup }}^{n m}$ is the dual space of $\mathbb{L}_{1}^{n m}$ (Stein and Shakarchi, 2011, Theorem 4.1), $\mathbb{H}_{\text {sup }}^{n, m}$ is the dual space of $\mathbb{H}_{1}^{n, m}$ and, furthermore, for every bounded linear functional $F$ on $\mathbb{H}_{1}^{n, m}$, there exists a unique $U \in \mathbb{H}_{\text {sup }}^{n, m}$ such that for all $V \in \mathbb{H}_{1}^{n, m}$,

$$
F(V)=\int_{\mathcal{S}} \operatorname{tr}\left(V(t)^{*} U(t)\right) \mu(d t)
$$

(Stein and Shakarchi, 2011, Theorem 4.1). From this, the bounded bilinear operator $\langle\cdot ; \cdot\rangle$ on $\mathbb{H}_{1}^{n} \times \mathbb{H}_{\text {sup }}^{n}$ with values in $\mathbb{C}$ is defined as follows. For $V \in \mathbb{H}_{1}^{n}, U \in \mathbb{H}_{\text {sup }}^{n}$, set

$$
\langle V ; U\rangle:=\int_{\mathcal{S}} \operatorname{tr}\left(V(t)^{*} U(t)\right) \mu(d t)
$$

Since $\|U(t)\| \leq\|U\|_{\text {sup }} \mu$-almost everywhere in $\mathcal{S}, t \in \mathcal{S}$, it follows from (3.2) that

$$
\begin{aligned}
|\langle V ; U\rangle| & =\left|\int_{\mathcal{S}} \operatorname{tr}\left(V(t)^{*} U(t)\right) \mu(d t)\right| \leq \int_{\mathcal{S}}\left|\operatorname{tr}\left(V(t)^{*} U(t)\right)\right| \mu(d t) \\
& \leq n \int_{\mathcal{S}}\|V(t)\|\|U(t)\| \mu(d t) \\
& \leq n\|U\|_{\text {sup }} \int_{\mathcal{S}}\|V(t)\| \mu(d t) \\
& =n\|U\|_{\text {sup }}\|V\|_{1},
\end{aligned}
$$

which shows that indeed $\langle\cdot ; \cdot\rangle$ is a bounded bilinear operator.

Next the operators $T \in \mathbb{B}\left(\mathbb{H}_{1}^{1}\right)$ and $\mathcal{G} \in \mathbb{B}\left(\mathbb{H}_{\text {sup }}^{1}\right)$ are defined. For any $\eta \in \mathbb{H}_{1}^{1}$,

$$
T \eta(\ell):=\int_{\mathcal{S}} \eta(t) g(t, \ell) \mu(d t)
$$

and for any $f \in \mathbb{H}_{\text {sup }}^{1}$,

$$
\mathcal{G} f(t):=\int_{\mathcal{S}} f(\ell) g(t, \ell) \mu(d \ell)
$$

Recall that $\langle\cdot ; \cdot\rangle$ stands for the bilinear form defined in (3.3). It is easy to see from (3.3) that

$$
\langle T \eta ; f\rangle=\langle\eta ; \mathcal{G} f\rangle
$$


and from (3.4) that

$$
|\langle\eta ; f\rangle| \leq\|f\|_{\text {sup }}\|\eta\|_{1}
$$

The adjoint $\mathcal{T}^{*} \in \mathbb{B}\left(\mathbb{H}_{\text {sup }}^{n}\right)$ of an operator $\mathcal{T} \in \mathbb{B}\left(\mathbb{H}_{1}^{n}\right)$ is defined by the relation

$$
\langle\mathcal{T}(V) ; U\rangle=\left\langle V ; \mathcal{T}^{*}(U)\right\rangle
$$

for every $V \in \mathbb{H}_{1}^{n}, U \in \mathbb{H}_{\text {sup }}^{n}$ (Hernández-Lerma and Lasserre, 1999, p. 208).

Proposition 3.1.3. Let $\mathcal{T} \in \mathbb{B}\left(\mathbb{H}_{1}^{n}\right)$ and $\mathcal{T}^{*} \in \mathbb{B}\left(\mathbb{H}_{\text {sup }}^{n}\right)$ be an operator and its adjoint respectively. Then $r_{\sigma}(\mathcal{T})=r_{\sigma}\left(\mathcal{T}^{*}\right)$.

Proof. As pointed out in (Damm, 2004, Chapter 3), $\sigma(\mathcal{T})=\overline{\sigma\left(\mathcal{T}^{*}\right)}$, and thus $r_{\sigma}(\mathcal{T})=$ $r_{\sigma}\left(\mathcal{T}^{*}\right)$

Define the following subspaces:

$$
\begin{aligned}
\mathbb{H}_{1}^{n *} & :=\left\{P \in \mathbb{H}_{1}^{n} ; P(t) \text { is Hermitian, } \forall t \in \mathcal{S}\right\}, \\
\mathbb{H}_{\text {sup }}^{n *} & :=\left\{P \in \mathbb{H}_{\text {sup }}^{n} ; P(t) \text { is Hermitian, } \forall t \in \mathcal{S}\right\}, \\
\mathbb{H}_{1}^{n+} & :=\left\{P \in \mathbb{H}_{1}^{n} ; P(t) \geq 0, \forall t \in \mathcal{S}\right\}, \\
\mathbb{H}_{\text {sup }}^{n+} & :=\left\{P \in \mathbb{H}_{\text {sup }}^{n} ; P(t) \geq 0, \forall t \in \mathcal{S}\right\}, \\
\widehat{\mathbb{H}}_{\text {sup }}^{q, p} & :=\left\{P \in \mathbb{H}_{\text {sup }}^{q, p} ; P(t) P(t)^{*} \geq \psi I \mu \text {-almost everywhere for } t \in \mathcal{S}, \text { for some } \psi>0\right\} .
\end{aligned}
$$

Similarly as in Remark 3.2.1 by Damm (2004), $\mathbb{H}_{1}^{n+}$ is a pointed closed, normal, solid convex cone in $\mathbb{H}_{1}^{n *}$, and its dual cone is $\mathbb{H}_{\text {sup }}^{n+}$ in $\mathbb{H}_{\text {sup }}^{n *}$ (Damm, 2004, Definition 3.1.1). An operator $\mathcal{T} \in \mathbb{B}\left(\mathbb{H}_{1}^{n *}\right)$ is called positive if $\mathcal{T}(V) \in \mathbb{H}_{1}^{n+}$ whenever $V \in \mathbb{H}_{1}^{n+}$, and similar definition applies to $\mathcal{T} \in \mathbb{B}\left(\mathbb{H}_{\text {sup }}^{n *}\right)$, that is, an operator $\mathcal{T} \in \mathbb{B}\left(\mathbb{H}_{\text {sup }}^{n *}\right)$ is called positive if $\mathcal{T}(V) \in \mathbb{H}_{\text {sup }}^{n+}$ whenever $V \in \mathbb{H}_{\text {sup }}^{n+}$.

The following result, that is employed in the next sections, is presented in a more general form by Damm (2004, Theorem 3.2.3) and is based on the theorem of Krein and Rutman. As pointed out by Damm (2004), its proof can be found in (Shaefer, 1971, Appendix 2.6) or in (Krasnoselskij et al., 1989, Theorem 9.11).

Lemma 3.1.4. Assume that $\mathcal{T} \in \mathbb{B}\left(\mathbb{H}_{1}^{n *}\right)$. If $\mathcal{T}$ is positive then there exists $U \in \mathbb{H}_{\text {sup }}^{n+}$, $U \neq 0$, such that $\mathcal{T}^{*}(U)=r_{\sigma}(\mathcal{T}) U$.

Next, a last remark regarding notation is presented. Assume that $\mathrm{T}$ is the discrete-time set. 
Remark 3.1.5. In the next chapters, when dealing with iterative maps $V(k, \ell), \ell \in \mathcal{S}$, $k \in \mathrm{T}$, on the Borel space $\mathcal{S}$ with $\sigma$-finite measure $\mu$, by a slight abuse of notation and for simplicity, it is denoted $V(k)$ meaning $V(k, \ell)$.

\subsection{Probabilistic Space}

This section introduces the probabilistic space that is considered throughout this thesis. The models of the jump stochastic systems that are studied in the present thesis are written in the state-space form. At each discrete-time instant $k \in \mathrm{T}$, assume that the jump variable $\theta(k)$ takes value in $\mathcal{S}$ and the remaining systems' input variables take value in $\tilde{\Omega}_{k}$. Recall from Remark 3.1.2 that $\mathcal{S}$ is a Borel space and $\mu$ is a $\sigma$-finite measure on $\mathcal{S}$.

Let $(\Omega, \mathcal{F})$ be a sample space, such that

$$
\Omega:=\prod_{k \in T}\left(\tilde{\Omega}_{k} \times \mathcal{S}_{k}\right)
$$

where $\times$ and $\prod$ denote the product space, $\mathcal{S}_{k}$ are copies of $\mathcal{S}, \mathrm{T}$ represents the discretetime set, and $\mathcal{F}$ is the associated product $\sigma$-algebra,

$$
\mathcal{F}:=\sigma\left\{\prod_{k \in \mathrm{T}} S_{k} \times \psi_{k} ; S_{k} \in \mathcal{B}\left(\tilde{\Omega}_{k}\right) \text { and } \psi_{k} \in \mathcal{B}(\mathcal{S}), \text { for } k \in \mathrm{T}\right\}
$$

Note that an element $\omega \in \Omega$ is such that $\omega=\{(\xi(k), \beta(k)) ; k \in \mathrm{T}\}$, where $\xi(k) \in \tilde{\Omega}_{k}$ and $\beta(k) \in \mathcal{S}$. Then for each $k \in \mathrm{T}$, set the random variable $\theta(k)$ from $\Omega$ to $\mathcal{S}$ as $\theta(k)(\omega)=\beta(k)$.

For $\mathrm{T}_{k}=\{i \in \mathrm{T} ; i \leq k\}$, define

$$
\mathcal{F}_{k}:=\sigma\left\{\prod_{l \in \mathrm{T}_{k}} S_{l} \times \psi_{l} \times \prod_{\tau=k+1}^{\infty} \tilde{\Omega}_{\tau} \times \mathcal{S} ; S_{l} \in \mathcal{B}\left(\tilde{\Omega}_{k}\right) \text { and } \psi_{l} \in \mathcal{B}(\mathcal{S}), \text { for } l \in \mathrm{T}_{k}\right\}
$$

so that $\mathcal{F}_{k} \subset \mathcal{F}$.

Define then the filtered probability space $\left(\Omega, \mathcal{F},\left\{\mathcal{F}_{k}\right\}_{k \in \mathrm{T}}, \mathcal{P}\right)$, where $\mathcal{P}$ is a probability measure such that, for $k \in \mathrm{T}$, and for each $\ell \in \mathcal{S}$ and $B \in \mathcal{B}(\mathcal{S})$,

$$
\mathcal{P}\left(\theta(k+1) \in B \mid \mathcal{F}_{k}\right)=\mathcal{P}(\theta(k+1) \in B \mid \theta(k)=\ell)
$$

The definition of a (Markov) transition probability function, also called stochastic 
kernel or transition probability kernel, is stated next (Hernández-Lerma and Lasserre, 2003, p. 22).

Definition 3.2.1. - Stochastic Kernel: Consider $\mathcal{X}, \mathcal{Y}$ Borel spaces. A stochastic kernel on $\mathcal{X}$ given $\mathcal{Y}$ is a function $P(\cdot, \cdot)$ such that

i. $P(y, \cdot)$ is a probability measure on $\mathcal{X}$ for each fixed $y \in \mathcal{Y}$; and

ii. $P(\cdot, B)$ is a measurable function on $\mathcal{Y}$ for each fixed $B \in \mathcal{B}(\mathcal{X})$.

The family of all stochastic kernels on $\mathcal{X}$ given $\mathcal{Y}$ is denoted by $\mathbb{P}(\mathcal{Y}, \mathcal{X})$.

Throughout the present thesis it is assumed that $\mathcal{G}(\cdot, \cdot)$ is a fixed $\mu$-continuous transition probability function having a probability density $g(\cdot, \cdot)$ with respect to $\mu$, so that, for each $\ell \in \mathcal{S}$ and $B \in \mathcal{B}(\mathcal{S})$,

$$
\mathcal{G}(\ell, B)=\int_{B} g(\ell, t) \mu(d t)
$$

where $g(\cdot, \cdot): \mathcal{S} \times \mathcal{S} \rightarrow \mathbb{R}^{+}$is a given nonnegative, measurable function, such that $\int_{\mathcal{S}} g(\ell, t) \mu(d t)=1$. It is also assumed that

$$
\mathcal{P}\left(\theta(k+1) \in B \mid \mathcal{F}_{k}\right)=\mathcal{P}(\theta(k+1) \in B \mid \theta(k)=\ell)=\mathcal{G}(\ell, B)
$$

The random process $\{\theta(k) ; k \in \mathrm{T}\}$ follows the Markov property, stated in 3.12, for each $k \in \mathrm{T}$. Clearly it is a $\mathcal{S}$-valued, discrete-time, time-homogeneous Markov chain. The initial probability measure on $\mathcal{S}$ for $\theta(0)=\theta_{0}$ is denoted by $\widehat{\mu}_{\mathcal{S}}$, so that for all $B \in \mathcal{B}(\mathcal{S})$,

$$
\mathcal{P}\left(\theta_{0} \in B\right)=\widehat{\mu}_{\mathcal{S}}(B)
$$

In most part of this thesis it is assumed that $\widehat{\mu}_{\mathcal{S}}$ is absolutely continuous with respect to $\mu$ as stated next.

Assumption 3.2.2. It holds that $\widehat{\mu}_{\mathcal{S}} \ll \mu$. Thus, from the Radon-Nikodym theorem, there exists $\nu \in \mathbb{H}_{1}^{1+}$ such that for all $B \in \mathcal{B}(\mathcal{S})$,

$$
\widehat{\mu}_{\mathcal{S}}(B)=\int_{B} \nu(\ell) \mu(d \ell)
$$

Remark 3.2.3. The Assumption 3.2.2 is not assumed in Section 6.1 when dealing with the finite-horizon minimum mean square linear Markov jump filter and in Section 7.1 in 
the study of the finite-horizon LQ optimal control problem with partial information. In these sections it is only assumed the existence of $\widehat{\mu}_{\mathcal{S}}$, the initial probability measure on $\mathcal{S}$ for $\theta_{0}$.

For any second-order $\mathcal{F}$-measurable random vector $r$ taking values in $\mathbb{C}^{n}$, define

$$
\|r\|_{2}^{2}=E\left(\|r\|^{2}\right)
$$

and denote by $\mathcal{C}^{n}$ the set of all sequences $r=\left\{r(k) ; k \in \mathrm{T}, r(k) \in \mathbb{C}^{n}\right\}$ such that

$$
\|r\|_{2}^{2}=\sum_{k \in T} E\left(\|r(k)\|^{2}\right)<\infty
$$

and that $r(k)$ is a $\mathcal{F}_{k}$-measurable random vector. Finally for $r, s \in \mathcal{C}^{n}$, define the bounded bilinear operator $\langle\cdot ; \cdot\rangle$ on $\mathcal{C}^{n} \times \mathcal{C}^{n}$ with values in $\mathbb{C}$ as

$$
\langle r ; s\rangle=\sum_{k \in \mathrm{T}} E\left(r(k)^{*} v(k)\right)
$$

\subsection{Discrete-Time MJLS with Markov Chain in a General State Space}

Consider the filtered probability space $\left(\Omega, \mathcal{F},\left\{\mathcal{F}_{k}\right\}_{k \in \mathrm{T}}, \mathcal{P}\right)$ introduced in Section 3.2 and recall that $\{\theta(k) ; k \in \mathrm{T}\}$ is a $\mathcal{S}$-valued, discrete-time, time-homogeneous Markov chain. A Markov jump linear system with Markov chain in a general state space is a dynamic system that can be described by the following model,

$$
\left\{\begin{aligned}
x(k+1) & =A(\theta(k)) x(k)+B(\theta(k)) u(k)+G(\theta(k)) w(k) \\
y(k) & =L(\theta(k)) x(k)+H(\theta(k)) w(k) \\
z(k) & =C(\theta(k)) x(k)+D(\theta(k)) u(k),
\end{aligned}\right.
$$

with initial condition $x(0)=x_{0}, \theta(0)=\theta_{0}$, and $\left(x_{0}, \theta_{0}\right)$ having distribution $\widehat{\mu}$, for some probability measure $\widehat{\mu}$ on $\mathbb{C}^{n} \times \mathcal{S}$. Also, denote by $\widehat{\mu}_{\mathcal{S}}$ the marginal of $\widehat{\mu}$ on $\mathcal{S}$, that is, $\widehat{\mu}_{\mathcal{S}}(B)=\widehat{\mu}\left(\mathbb{C}^{n} \times B\right)$, for all $B \in \mathcal{B}(\mathcal{S})$, such that $\widehat{\mu}_{\mathcal{S}}$ is the initial probability measure on $\mathcal{S}$ for $\theta_{0}$ as stated in (3.13). Finally assume that $E\left(x_{0}\right):=\zeta, E\left(x_{0} x_{0}^{*}\right):=\mathbb{Q}_{0}$, and thus $E\left(\left\|x_{0}-\zeta\right\|^{2}\right)=\operatorname{tr}\left(\mathbb{Q}_{0}-\zeta \zeta^{*}\right)<\infty$. These hypotheses are assumed to hold for all MJLS considered in this thesis. Recall that, as stated in Remark 3.2.3, the Assumption 3.2.2 is assumed in most part of this thesis, but is not assumed in Sections 6.1 and 7.1. 
Consider a discrete-time instant $k \in \mathrm{T}$. As usual, $x(k) \in \mathbb{C}^{n}$ represents the state variable of the system, $u(k) \in \mathbb{C}^{m}$ the control variable, $w(k) \in \mathbb{C}^{q}$ the noise sequence acting on the system, $y(k) \in \mathbb{C}^{p}$ the measurable variable available to the controller, $z(k) \in \mathbb{C}^{r}$ the output of the system, and $\theta(k) \in \mathcal{S}$ the operation mode of the system, also called jump variable or jump parameter. Notice that under a given operation mode $\theta(k)$, the dynamic system (3.15) evolves as a discrete-time, linear, time-invariant system. This model, or some of its variants, is considered throughout this thesis, when studying several control problems. 


\section{Chapter 4}

\section{Stochastic Stability}

This chapter deals with the stochastic stability (SS) for MJLS with Markov chain in a Borel space $\mathcal{S}$. Necessary and sufficient conditions for SS are presented in Theorem 4.3.3. It is shown that SS is equivalent to the spectral radius of a bounded linear operator in a Banach space being less than 1 or to the existence of a solution to a "Lyapunov-like" equation. Furthermore, some easy-to-check sufficient conditions for SS are presented in Corollaries 4.3.4 and 4.3.5.

\subsection{Stochastic Stability Definition}

In this section the stochastic stability of a discrete-time MJLS with Markov chain taking values in a general state space is defined. In order to introduce the SS concept it suffices to deal with the homogeneous MJLS, which is a variant of the MJLS model presented in Section 3.3.

On the filtered probability space $\left(\Omega, \mathcal{F},\left\{\mathcal{F}_{k}\right\}_{k \in \mathbb{N}}, \mathcal{P}\right)$ consider the following discretetime Markov jump linear system

$$
x(k+1)=\Gamma(\theta(k)) x(k) .
$$

Recall from Section 3.3 that $E\left(\left\|x_{0}\right\|^{2}\right)<\infty,\left(x_{0}, \theta_{0}\right)$ has a distribution $\widehat{\mu}$, for some probability measure $\widehat{\mu}$ on $\mathbb{C}^{n} \times \mathcal{S}$, and the marginal of $\widehat{\mu}$ on $\mathcal{S}$ is denoted by $\widehat{\mu}_{\mathcal{S}}$. Recall also that from Assumption 3.2.2 and the Radon-Nikodym theorem there exists a measurable function $\nu$ from $\mathcal{S}$ into $\mathbb{R}^{+}$such that for all $B \in \mathcal{B}(\mathcal{S})$,

$$
\widehat{\mu}_{\mathcal{S}}(B)=\int_{B} \nu(\ell) \mu(d \ell)
$$


as stated in (3.14). Furthermore, system (4.1) follows the assumption stated next that requires the system's parameter to be bounded (in the ess-sup sense).

Assumption 4.1.1. $\Gamma \in \mathbb{H}_{\text {sup }}^{n}$.

From Proposition D.8 in (Hernández-Lerma and Lasserre, 1996, p. 184), there exists a stochastic kernel $\mathcal{K}(\cdot, \cdot) \in \mathbb{P}\left(\mathcal{S}, \mathbb{C}^{n}\right)$ such that for all $C \in \mathcal{B}\left(\mathbb{C}^{n}\right)$ and $B \in \mathcal{B}(\mathcal{S})$,

$$
\widehat{\mu}(C \times B)=\int_{B} \mathcal{K}(\ell, C) \widehat{\mu}_{\mathcal{S}}(d \ell)
$$

Combining (3.14) and (4.2) one gets that for all $C \in \mathcal{B}\left(\mathbb{C}^{n}\right)$ and $B \in \mathcal{B}(\mathcal{S})$,

$$
\widehat{\mu}(C \times B)=\int_{B} \mathcal{K}(\ell, C) \nu(\ell) \mu(d \ell) .
$$

From (4.3) and recalling that $E\left(\left\|x_{0}\right\|^{2}\right)<\infty$ it can be concluded that

$$
E\left(\left\|x_{0}\right\|^{2}\right)=\int_{\mathcal{S}} \int_{\mathbb{C}^{n}}\|u\|^{2} \widehat{\mu}(d u, d \ell)=\int_{\mathcal{S}} \int_{\mathbb{C}^{n}}\|u\|^{2} \mathcal{K}(\ell, d u) \nu(\ell) \mu(d \ell)<\infty .
$$

Define the mapping $R=\{R(\ell) ; \ell \in \mathcal{S}\}$ where for each $\ell \in \mathcal{S}$,

$$
R(\ell):=\nu(\ell) \int_{\mathbb{C}^{n}} u u^{*} \mathcal{K}(\ell, d u)
$$

Notice that from (4.4) and (4.5),

$$
\begin{aligned}
\|R\|_{1} & =\int_{\mathcal{S}}\|R(\ell)\| \mu(d \ell)=\int_{\mathcal{S}}\left\|\int_{\mathbb{C}^{n}} u u^{*} \mathcal{K}(\ell, d u) \nu(\ell)\right\| \mu(d \ell) \\
& \leq \int_{\mathcal{S}} \int_{\mathbb{C}^{n}}\|u\|^{2} \mathcal{K}(\ell, d u) \nu(\ell) \mu(d \ell)=E\left(\left\|x_{0}\right\|^{2}\right) \\
& <\infty
\end{aligned}
$$

and one can conclude that $R \in \mathbb{H}_{1}^{n+}$.

The stability concept that is considered in this thesis is stated next.

Definition 4.1.2. - Stochastically Stability (SS): System (4.1) is said stochastically stable $(S S)$ if

$$
\sum_{k=0}^{\infty} E\left(\|x(k)\|^{2}\right)<\infty
$$

for any initial distribution $\widehat{\mu}$ satisfying $E\left(\left\|x_{0}\right\|^{2}\right)<\infty$ and Assumption 3.2.2. System 
(4.1) is said $S S$ for real initial state variables $x_{0}$ if $\sum_{k=0}^{\infty} E\left(\|x(k)\|^{2}\right)<\infty$ for any initial distribution $\widehat{\mu}$ satisfying $E\left(\left\|x_{0}\right\|^{2}\right)<\infty$, Assumption 3.2.2, and $x_{0} \in \mathbb{R}^{n}$.

\subsection{Auxiliary Results}

In this section several auxiliary results and the main operators that are needed to characterize the "Lyapunov-like" equation and the SS results for system (4.1) are presented.

Consider the MJLS presented in (4.1). The linear operators $\mathcal{E}, \mathcal{L}$ and $\mathcal{T}$ are defined next. Recall from Assumption 4.1.1 that $\Gamma \in \mathbb{H}_{\text {sup }}^{n}$, and set for $V \in \mathbb{H}_{1}^{n}, U \in \mathbb{H}_{\text {sup }}^{n}, \ell \in \mathcal{S}$,

$$
\begin{aligned}
\mathcal{E}(U)(\ell) & :=\int_{\mathcal{S}} g(\ell, t) U(t) \mu(d t), \\
\mathcal{L}(V)(\ell) & :=\int_{\mathcal{S}} g(t, \ell) \Gamma(t) V(t) \Gamma(t)^{*} \mu(d t), \\
\mathcal{T}(U)(\ell) & :=\Gamma(\ell)^{*}\left(\int_{\mathcal{S}} g(\ell, t) U(t) \mu(d t)\right) \Gamma(\ell)=\Gamma(\ell)^{*} \mathcal{E}(U)(\ell) \Gamma(\ell) .
\end{aligned}
$$

Notice that $\mathcal{E}, \mathcal{L}$ and $\mathcal{T}$ are positive operators, that is, $\mathcal{E}(\widehat{U}) \geq \mathcal{E}(U)$ whenever $\widehat{U} \geq U$, and similarly for $\mathcal{L}$ and $\mathcal{T}$. These operators are the main operators necessary to characterize the SS for system (4.1).

Proposition 4.2.1. Operators $\mathcal{E}, \mathcal{L}$ and $\mathcal{T}$ are such that:

i) $\mathcal{E} \in \mathbb{B}\left(\mathbb{H}_{\text {sup }}^{n}\right)$ with $\|\mathcal{E}\| \leq 1$.

ii) $\mathcal{L} \in \mathbb{B}\left(\mathbb{H}_{1}^{n}\right)$ with $\|\mathcal{L}\| \leq\|\Gamma\|_{\text {sup }}^{2}$.

iii) $\mathcal{T} \in \mathbb{B}\left(\mathbb{H}_{\text {sup }}^{n}\right)$ with $\|\mathcal{T}\| \leq\|\Gamma\|_{\text {sup }}^{2}$.

Proof. For any $V \in \mathbb{H}_{1}^{n}, U \in \mathbb{H}_{\text {sup }}^{n}$, (i)-(iii) are proved below.

i) From (4.6) and $\|U(t)\| \leq\|U\|_{\text {sup }} \mu$-almost everywhere in $\mathcal{S}$, it follows that

$$
\begin{aligned}
\|\mathcal{E}(U)(\ell)\| & =\left\|\int_{\mathcal{S}} g(\ell, t) U(t) \mu(d t)\right\| \\
& \leq \int_{\mathcal{S}} g(\ell, t)\|U(t)\| \mu(d t) \\
& \leq\|U\|_{\text {sup }} \int_{\mathcal{S}} g(\ell, t) \mu(d t) \\
& =\|U\|_{\text {sup }},
\end{aligned}
$$

since $\int_{\mathcal{S}} g(\ell, t) \mu(d t)=1$. From (4.9) it results that $\|\mathcal{E}(U)\|_{\text {sup }} \leq\|U\|_{\text {sup }}$ and thus $\|\mathcal{E}\| \leq 1$. 
ii) From (4.7), Fubini's theorem, and $\|\Gamma(t)\| \leq\|\Gamma\|_{\text {sup }} \mu$-almost everywhere in $\mathcal{S}$, it results that

$$
\begin{aligned}
\|\mathcal{L}(V)\|_{1} & =\int_{\mathcal{S}}\|\mathcal{L}(V)(\ell)\| \mu(d \ell) \\
& =\int_{\mathcal{S}}\left\|\int_{\mathcal{S}} g(t, \ell) \Gamma(t) V(t) \Gamma(t)^{*} \mu(d t)\right\| \mu(d \ell) \\
& \leq \int_{\mathcal{S}} \int_{\mathcal{S}} g(t, \ell)\left\|\Gamma(t) V(t) \Gamma(t)^{*}\right\| \mu(d t) \mu(d \ell) \\
& \leq \int_{\mathcal{S}} \int_{\mathcal{S}} g(t, \ell)\|\Gamma(t)\|^{2}\|V(t)\| \mu(d t) \mu(d \ell) \\
& \leq\|\Gamma\|_{\sup }^{2} \int_{\mathcal{S}}\left(\int_{\mathcal{S}} g(t, \ell) \mu(d \ell)\right)\|V(t)\| \mu(d t) \\
& =\|\Gamma\|_{\sup }^{2} \int_{\mathcal{S}}\|V(t)\| \mu(d t) \\
& =\|\Gamma\|_{\sup }^{2}\|V\|_{1},
\end{aligned}
$$

recalling that $\int_{\mathcal{S}} g(t, \ell) \mu(d \ell)=1$. From (4.10) one can get that $\|\mathcal{L}\| \leq\|\Gamma\|_{\text {sup }}^{2}$. iii) From (4.8) and $\|U(t)\| \leq\|U\|_{\text {sup }} \mu$-almost everywhere in $\mathcal{S}$, it follows that

$$
\begin{aligned}
\|\mathcal{T}(U)(\ell)\| & =\left\|\Gamma(\ell)^{*}\left(\int_{\mathcal{S}} g(\ell, t) U(t) \mu(d t)\right) \Gamma(\ell)\right\| \\
& \leq\|\Gamma(\ell)\|^{2}\left\|\int_{\mathcal{S}} g(\ell, t) U(t) \mu(d t)\right\| \\
& \leq\|\Gamma(\ell)\|^{2} \int_{\mathcal{S}} g(\ell, t)\|U(t)\| \mu(d t) \\
& \leq\|\Gamma(\ell)\|^{2}\|U\|_{\text {sup }} \int_{\mathcal{S}} g(\ell, t) \mu(d t) \\
& \leq\|\Gamma(\ell)\|^{2}\|U\|_{\text {sup }},
\end{aligned}
$$

recalling again that $\int_{\mathcal{S}} g(\ell, t) \mu(d t)=1$. From (4.11) one gets that $\|\mathcal{T}(U)\|_{\text {sup }} \leq$ $\|\Gamma\|_{\text {sup }}^{2}\|U\|_{\text {sup }}$ and thus that $\|\mathcal{T}\| \leq\|\Gamma\|_{\text {sup }}^{2}$.

Proposition 4.2.2. For any $U \in \mathbb{H}_{\text {sup }}^{n+}$,

$$
E\left(x(k+1)^{*} U(\theta(k+1)) x(k+1)\right)=E\left(x(k)^{*} \Gamma(\theta(k))^{*} \mathcal{E}(U)(\theta(k)) \Gamma(\theta(k)) x(k)\right) .
$$

Proof. From (4.1) and (4.6), for any $U \in \mathbb{H}_{\text {sup }}^{n+}$, it follows that

$$
E\left(x(k+1)^{*} U(\theta(k+1)) x(k+1)\right)=E\left(E\left(x(k)^{*} \Gamma(\theta(k))^{*} U(\theta(k+1)) \Gamma(\theta(k)) x(k) \mid \mathcal{F}_{k}\right)\right)
$$




$$
\begin{aligned}
& =E\left(x(k)^{*} \Gamma(\theta(k))^{*} E\left(U(\theta(k+1)) \mid \mathcal{F}_{k}\right) \Gamma(\theta(k)) x(k)\right) \\
& =E\left(x(k)^{*} \Gamma(\theta(k))^{*}\left(\int_{\mathcal{S}} U(t) g(\theta(k), t) \mu(d t)\right) \Gamma(\theta(k)) x(k)\right) \\
& =E\left(x(k)^{*} \Gamma(\theta(k))^{*} \mathcal{E}(U)(\theta(k)) \Gamma(\theta(k)) x(k)\right) .
\end{aligned}
$$

Recall that $\langle\cdot ; \cdot\rangle$ stands for the bilinear form defined in (3.3). The next two propositions present important properties regarding the operators $\mathcal{L}$ and $\mathcal{T}$.

Proposition 4.2.3. $\mathcal{L}^{*}=\mathcal{T}$.

Proof. From Fubini's theorem, (3.3), (4.7) and (4.8) it can be obtained that, for every $V \in \mathbb{H}_{1}^{n}, U \in \mathbb{H}_{\text {sup }}^{n}$,

$$
\begin{aligned}
\langle\mathcal{L}(V) ; U\rangle & =\int_{\mathcal{S}} \operatorname{tr}\left\{\mathcal{L}(V)(\ell)^{*} U(\ell)\right\} \mu(d \ell) \\
& =\int_{\mathcal{S}} \operatorname{tr}\left\{\left(\int_{\mathcal{S}} g(t, \ell) \Gamma(t)^{*} V(t)^{*} \Gamma(t) \mu(d t)\right) U(\ell)\right\} \mu(d \ell) \\
& =\int_{\mathcal{S}}\left\{\int_{\mathcal{S}} g(t, \ell) \operatorname{tr}\left(\Gamma(t)^{*} V(t)^{*} \Gamma(t) U(\ell)\right) \mu(d t)\right\} \mu(d \ell) \\
& =\int_{\mathcal{S}}\left\{\int_{\mathcal{S}} g(t, \ell) \operatorname{tr}\left(V(t)^{*} \Gamma(t)^{*} U(\ell) \Gamma(t)\right) \mu(d \ell)\right\} \mu(d t) \\
& =\int_{\mathcal{S}} \operatorname{tr}\left\{V(t)^{*} \Gamma(t)^{*}\left(\int_{\mathcal{S}} g(t, \ell) U(\ell) \mu(d \ell)\right) \Gamma(t)\right\} \mu(d t) \\
& =\int_{\mathcal{S}} \operatorname{tr}\left\{V(t)^{*} \mathcal{T}(U)(t)\right\} \mu(d t) \\
& =\langle V ; \mathcal{T}(U)\rangle,
\end{aligned}
$$

completing the proof.

Proposition 4.2.4. $r_{\sigma}(\mathcal{L})=r_{\sigma}(\mathcal{T})$.

Proof. It is immediate from Propositions 3.1.3 and 4.2.3.

The last auxiliary result is presented next. It is a well known fact that every nonzero $\sigma$-finite measure is equivalent to a probability measure. In particular one can easily get the next proposition.

Proposition 4.2.5. There is a density $\nu_{1}$ with respect to $\mu$ such that $\nu_{1}(\ell)>0$ for each $\ell \in \mathcal{S}$ and $\int_{\mathcal{S}} \nu_{1}(\ell) \mu(d \ell)=1$. 
Proof. The case $\mu(\mathcal{S})<\infty$ is immediate since one can set

$$
\nu_{1}(\ell)=\frac{1}{\mu(\mathcal{S})}
$$

Suppose that $\mu(\mathcal{S})=\infty$. Since $\mu$ is $\sigma$-finite, there exists a countable sequence of sets $S_{k} \in \mathcal{B}(\mathcal{S})$ such that $\mathcal{S}=\cup_{k=1}^{\infty} S_{k}$ and $\mu\left(S_{k}\right)<\infty$. Without loss of generality, assume that $\mu\left(S_{1}\right)>0$ and $\mu\left(\cup_{i=1}^{k+1} S_{i}\right)>\mu\left(\cup_{i=1}^{k} S_{i}\right)$ for each $k=1,2, \ldots$ Then define the mutually disjoint sets $\widetilde{S}_{k}$ as $\widetilde{S}_{1}=S_{1}$ and $\widetilde{S}_{k}=S_{k} \cap\left(\cup_{i=1}^{k-1} S_{i}\right)^{c}, k=2,3, \ldots$, so that $\cup_{k=1}^{\iota} S_{k}=\cup_{k=1}^{\iota} \widetilde{S}_{k}$. Moreover $0<\mu\left(\widetilde{S}_{k}\right)<\infty$ holds. Define the density $\nu_{1}$, for $\ell \in \widetilde{S}_{k}$, $k=1,2, \ldots$, as

$$
\nu_{1}(\ell)=2^{-k} \frac{1}{\mu\left(\widetilde{S}_{k}\right)}
$$

Thus

$$
\int_{\mathcal{S}} \nu_{1}(\ell) \mu(d \ell)=\sum_{k=1}^{\infty} \int_{\widetilde{S}_{k}} 2^{-k} \frac{1}{\mu\left(\widetilde{S}_{k}\right)} \mu(d \ell)=\sum_{k=1}^{\infty} 2^{-k}=1,
$$

completing the proof.

\subsection{Conditions for Stochastic Stability}

In this section the necessary and sufficient conditions for the stochastic stability of the system (4.1) are derived. In Theorem 4.3.3 it is shown that SS is equivalent to the spectral radius of an operator being less than 1 or the existence of a solution to a "Lyapunov-like" equation. Some easy-to-check sufficient conditions for SS are presented in Corollaries 4.3.4 and 4.3.5.

Initially set, for $\ell \in \mathcal{S}, k \in \mathbb{N}$,

$$
\begin{aligned}
F(k+1, \ell) & :=E\left(x(k+1) x(k+1)^{*} g(\theta(k), \ell)\right) \\
F(0, \ell) & :=R(\ell),
\end{aligned}
$$

where $R \in \mathbb{H}_{1}^{n+}$ was defined in (4.5).

Recall from Section 3.2 that $\mathcal{F}_{k-1}=\sigma\left\{x_{0}, \theta_{0}, \ldots, x(k-1), \theta(k-1)\right\}$ is the $\sigma$-algebra generated by $\left\{x_{0}, \theta_{0}, \ldots, x(k-1), \theta(k-1)\right\}$. The next result establishes a link between $F(k, \ell)$ and the operator $\mathcal{L}$ defined in (4.7).

Proposition 4.3.1. For each $k \in \mathbb{N}, F(k)=\{F(k, \ell) ; \ell \in \mathcal{S}\} \in \mathbb{H}_{1}^{n+}$, and for all $\ell \in \mathcal{S}$,

$$
F(k+1, \ell)=\mathcal{L}(F(k))(\ell) .
$$


Proof. The proposition will be proved by induction on $k$. For $k=0$ it follows from (4.12) that

$$
F(0)=R \in \mathbb{H}_{1}^{n+}
$$

and from (4.12), (4.1)-(4.5), (4.7), that

$$
\begin{aligned}
F(1, \ell) & =E\left(x(1) x(1)^{*} g(\theta(0), \ell)\right)=E\left(\Gamma\left(\theta_{0}\right) x_{0} x_{0}^{*} \Gamma\left(\theta_{0}\right)^{*} g\left(\theta_{0}, \ell\right)\right) \\
& =\int_{\mathcal{S}} \int_{\mathbb{C}^{n}} \Gamma(s) u u^{*} \Gamma(s)^{*} g(s, \ell) \mathcal{K}(s, d u) \nu(s) \mu(d s) \\
& =\int_{\mathcal{S}} g(s, \ell) \Gamma(s)\left(\int_{\mathbb{C}^{n}} u u^{*} \mathcal{K}(s, d u) \nu(s)\right) \Gamma(s)^{*} \mu(d s) \\
& =\int_{\mathcal{S}} g(s, \ell) \Gamma(s) R(s) \Gamma(s)^{*} \mu(d s) \\
& =\mathcal{L}(R)(\ell),
\end{aligned}
$$

showing the result for $k=0$. Suppose that the result holds for $k>0$. From (4.1) and the Fubini's theorem, one gets that

$$
\begin{aligned}
& F(k+1, \ell)=E\left(x(k+1) x(k+1)^{*} g(\theta(k), \ell)\right) \\
& =E\left(E\left(\Gamma(\theta(k)) \Gamma(\theta(k-1)) x(k-1)(\Gamma(\theta(k)) \Gamma(\theta(k-1)) x(k-1))^{*} g(\theta(k), \ell) \mid \mathcal{F}_{k-1}\right)\right) \\
& =E\left(\int_{\mathcal{S}} \Gamma(s) \Gamma(\theta(k-1)) x(k-1)(\Gamma(s) \Gamma(\theta(k-1)) x(k-1))^{*} g(s, \ell) g(\theta(k-1), s) \mu(d s)\right) \\
& =\int_{\mathcal{S}} \Gamma(s) E\left(x(k) x(k)^{*} g(\theta(k-1), s)\right) \Gamma(s)^{*} g(s, \ell) \mu(d s) \\
& =\int_{\mathcal{S}} \Gamma(s) F(k, s) \Gamma(s)^{*} g(s, \ell) \mu(d s) \\
& =\mathcal{L}(F(k))(\ell) .
\end{aligned}
$$

Since $\mathcal{L} \in \mathbb{B}\left(\mathbb{H}_{1}^{n}\right)$ from Proposition 4.2.1 and it is a positive operator, it follows from the induction hypothesis that $F(k) \in \mathbb{H}_{1}^{n+}$ and from (4.15) that $F(k+1, \ell) \geq 0$ and $F(k+1)=\mathcal{L}(F(k)) \in \mathbb{H}_{1}^{n+}$.

Define, next, $Q(k), k \in \mathbb{N}$ as,

$$
Q(k):=E\left(x(k) x(k)^{*}\right) .
$$

Then, the next result follows. 
Proposition 4.3.2. For every $k \in \mathbb{N}$,

$$
Q(k)=\int_{\mathcal{S}} F(k, \ell) \mu(d \ell)
$$

Proof. For $k=0$ it follows from (4.2)-(4.5), (4.12), that

$$
\begin{aligned}
Q(0) & =E\left(x_{0} x_{0}^{*}\right)=\int_{\mathcal{S}} \int_{\mathbb{C}^{n}} u u^{*} \widehat{\mu}(d u, d \ell) \\
& =\int_{\mathcal{S}} \int_{\mathbb{C}^{n}} u u^{*} \mathcal{K}(\ell, d u) \nu(\ell) \mu(d \ell)=\int_{\mathcal{S}} R(\ell) \mu(d \ell)=\int_{\mathcal{S}} F(0, \ell) \mu(d \ell) .
\end{aligned}
$$

Suppose the result holds for $k>0$. From Fubini's theorem, (4.1), (4.7), (4.12) and Proposition 4.3.1 one can conclude that

$$
\begin{aligned}
Q(k+1) & =E\left(x(k+1) x(k+1)^{*}\right)=E\left(E\left(\Gamma(\theta(k)) x(k) x(k)^{*} \Gamma(\theta(k))^{*} \mid \mathcal{F}_{k-1}\right)\right) \\
& =E\left(\int_{\mathcal{S}} g(\theta(k-1), s) \Gamma(s) x(k) x(k)^{*} \Gamma(s)^{*} \mu(d s)\right) \\
& =\int_{\mathcal{S}} \Gamma(s) E\left(g(\theta(k-1), s) x(k) x(k)^{*}\right) \Gamma(s)^{*} \mu(d s) \\
& =\int_{\mathcal{S}} g(s, t) \int_{\mathcal{S}} \Gamma(s) F(k, s) \Gamma(s)^{*} \mu(d s) \mu(d t) \\
& =\int_{\mathcal{S}}\left(\int_{\mathcal{S}} g(s, t) \Gamma(s) F(k, s) \Gamma(s)^{*} \mu(d s)\right) \mu(d t) \\
& =\int_{\mathcal{S}} \mathcal{L}(F(k))(t) \mu(d t)=\int_{\mathcal{S}} F(k+1, t) \mu(d t),
\end{aligned}
$$

completing the proof.

The main result of this chapter is presented next. It states that SS is equivalent to the spectral radius of the bounded linear operator $\mathcal{L}$ being less than 1 or to the existence of a solution to the "Lyapunov-like" equation (4.17). This equation is referred to as "Lyapunov-like" equation, because it plays a similar role as the (discrete-time) Lyapunov equation associated with the stability analysis for discrete-time time-invariant-linear systems (see Callier and Desoer, 1991, p. 214).

Theorem 4.3.3. The following assertions are equivalent:

i) System (4.1) is $S S$.

ii) System (4.1) is SS for real initial state variables $x_{0}$.

iii) $r_{\sigma}(\mathcal{L})<1$. 
iv) Given any $V \in \mathbb{H}_{\text {sup }}^{n+}$ such that $V(t) \geq \alpha I \mu$-almost everywhere for $t \in \mathcal{S}$, for some $\alpha>0$, there exists $S \in \mathbb{H}_{\text {sup }}^{n+}$ such that $\mu$-almost everywhere in $\mathcal{S}$,

$$
S(\ell)-\mathcal{T}(S)(\ell)=V(\ell)
$$

v) There exists $S \in \mathbb{H}_{\text {sup }}^{n+}$ and $\epsilon>0$ such that $\mu$-almost everywhere in $\mathcal{S}$,

$$
S(\ell)-\mathcal{T}(S)(\ell) \geq \epsilon I
$$

Proof. iii) $\Longrightarrow$ i): From Lemma 1 by Kubrusly (1985) one gets that $r_{\sigma}(\mathcal{L})<1$ implies that there exists $\gamma \geq 1,0<\rho<1$, such that $\left\|\mathcal{L}^{k}\right\| \leq \gamma \rho^{k}$ for every $k \in \mathbb{N}$. From $(4.13)$ it follows that $F(k)=\mathcal{L}^{k}(R)$, and from (4.16) one gets that $Q(k)=\int_{\mathcal{S}} \mathcal{L}^{k}(R)(\ell) \mu(d \ell)$. Therefore,

$$
\begin{aligned}
E\left(\|x(k)\|^{2}\right) & =E\left(\operatorname{tr}\left(x(k) x(k)^{*}\right)\right)=\operatorname{tr}\left(E\left(x(k) x(k)^{*}\right)\right)=\operatorname{tr}(Q(k)) \\
& =\operatorname{tr}\left(\int_{\mathcal{S}} \mathcal{L}^{k}(R)(\ell) \mu(d \ell)\right)=\int_{\mathcal{S}} \operatorname{tr}\left(\mathcal{L}^{k}(R)(\ell)\right) \mu(d \ell) \\
& \leq n \int_{\mathcal{S}}\left\|\mathcal{L}^{k}(R)(\ell)\right\| \mu(d \ell)=n\left\|\mathcal{L}^{k}(R)\right\|_{1} \\
& \leq n\left\|\mathcal{L}^{k}\right\|\|R\|_{1} \leq n\|R\|_{1} \gamma \rho^{k}
\end{aligned}
$$

and from (4.19) it follows that $\sum_{k=0}^{\infty} E\left(\|x(k)\|^{2}\right)<\infty$ for any initial distribution $\widehat{\mu}$ satisfying $E\left(\left\|x_{0}\right\|^{2}\right)<\infty$ and Assumption 3.2.2 so that, according to Definition 4.1.2, system (4.1) is $\mathrm{SS}$.

i) $\Longrightarrow$ ii): This is immediate.

ii) $\Longrightarrow$ iii): Initially it will be shown that for arbitrary $P \in \mathbb{H}_{1}^{n+}$,

$$
\sum_{k=0}^{\infty}\left\|\mathcal{L}^{k}(P)\right\|_{1}<\infty
$$

Set $P^{r}(\ell)=\|P(\ell)\| I, \ell \in \mathcal{S}$. Clearly $P^{r} \in \mathbb{H}_{1}^{n+}, P^{r} \geq P$, and $\left\|P^{r}\right\|_{1}=\|P\|_{1}$. From Proposition 4.2 .5 one can find a density $\nu$ with respect to $\mu$ such that $\nu(\ell)>0$ for every $\ell \in \mathcal{S}$ and $\int_{\mathcal{S}} \nu(\ell) \mu(d \ell)=1$. Consider a null mean random vector $\bar{x}_{0}(\ell)$ with covariance matrix $\|P(\ell)\| I$ and define the initial condition $\left(x_{0}, \theta_{0}\right)$ for system (4.1) as follows: $\theta_{0}$ has density $\nu$ with respect to $\mu$ on $\mathcal{S}$, and $x_{0}=\frac{1}{\nu\left(\theta_{0}\right)^{1 / 2}} \bar{x}_{0}\left(\theta_{0}\right)$. From this definition it results 
that $\nu=\left(d \widehat{\mu}_{\mathcal{S}} / d \mu\right), x_{0} \in \mathbb{R}^{n}$ and, recalling that $E\left(\bar{x}_{0}\left(\theta_{0}\right) \bar{x}_{0}\left(\theta_{0}\right)^{\prime} \mid \theta_{0}\right)=\left\|P\left(\theta_{0}\right)\right\| I$, that

$$
\begin{aligned}
E\left(x_{0} x_{0}^{*}\right) & =E\left(E\left(x_{0} x_{0}^{*} \mid \theta_{0}\right)\right)=E\left(\frac{1}{\nu\left(\theta_{0}\right)} E\left(\bar{x}_{0}\left(\theta_{0}\right) \bar{x}_{0}\left(\theta_{0}\right)^{\prime} \mid \theta_{0}\right)\right)=E\left(\frac{1}{\nu\left(\theta_{0}\right)}\left\|P\left(\theta_{0}\right)\right\| I\right) \\
& =\int_{\mathcal{S}} \frac{1}{\nu(\ell)}\|P(\ell)\| \nu(\ell) \mu(d \ell) I=\int_{\mathcal{S}}\|P(\ell)\| \mu(d \ell) I \\
& =\|P\|_{1} I
\end{aligned}
$$

so that from (4.21), $E\left(\left\|x_{0}\right\|^{2}\right)=E\left(\operatorname{tr}\left(x_{0} x_{0}^{*}\right)\right)=n\|P\|_{1}<\infty$. Therefore the assumption that $E\left(\left\|x_{0}\right\|^{2}\right)<\infty$ and the Assumption 3.2.2 are satisfied with $x_{0} \in \mathbb{R}^{n}$. Moreover, $R(\ell)=E\left(x_{0} x_{0}^{*} \mid \theta_{0}=\ell\right) \nu(\ell)=\|P(\ell)\| I=P^{r}(\ell)$. From the fact that $\mathcal{L}^{k}(P) \leq \mathcal{L}^{k}\left(P^{r}\right)$, (4.13), (4.16) and the SS (Definition 4.1.2), it follows that

$$
\begin{aligned}
\sum_{k=0}^{\infty}\left\|\mathcal{L}^{k}(P)\right\|_{1} & \leq \sum_{k=0}^{\infty}\left\|\mathcal{L}^{k}\left(P^{r}\right)\right\|_{1}=\sum_{k=0}^{\infty} \int_{\mathcal{S}}\left\|\mathcal{L}^{k}\left(P^{r}\right)(\ell)\right\| \mu(d \ell) \\
& \leq \sum_{k=0}^{\infty} \int_{\mathcal{S}} \operatorname{tr}\left(\mathcal{L}^{k}\left(P^{r}\right)(\ell)\right) \mu(d \ell)=\sum_{k=0}^{\infty} \operatorname{tr}\left(\int_{\mathcal{S}} \mathcal{L}^{k}\left(P^{r}\right)(\ell) \mu(d \ell)\right) \\
& =\sum_{k=0}^{\infty} \operatorname{tr}\left(\int_{\mathcal{S}} F(k, \ell) \mu(d \ell)\right)=\sum_{k=0}^{\infty} \operatorname{tr}(Q(k)) \\
& =\sum_{k=0}^{\infty} E\left(\operatorname{tr}\left(x(k) x(k)^{*}\right)\right)=\sum_{k=0}^{\infty} E\left(\|x(k)\|^{2}\right)<\infty .
\end{aligned}
$$

Consider now an arbitrary $W \in \mathbb{H}_{1}^{n}$. From Remark 3.1.1, it can be written $W=\left(W^{1}-\right.$ $\left.W^{2}\right)+\sqrt{-1}\left(W^{3}-W^{4}\right)$, with $W^{i} \in \mathbb{H}_{1}^{n+}$. From this and (4.22) it follows that

$$
\sum_{k=0}^{\infty}\left\|\mathcal{L}^{k}(W)\right\|_{1} \leq \sum_{k=0}^{\infty} \sum_{i=1}^{4}\left\|\mathcal{L}^{k}\left(W^{i}\right)\right\|_{1}=\sum_{i=1}^{4} \sum_{k=0}^{\infty}\left\|\mathcal{L}^{k}\left(W^{i}\right)\right\|_{1}<\infty
$$

Since (4.23) holds for every $W \in \mathbb{H}_{1}^{n}$ it follows from Lemma 1 by Kubrusly (1985) that $r_{\sigma}(\mathcal{L})<1$.

iv) $\Longrightarrow v)$ : This is immediate.

$v) \Longrightarrow i$ ): Consider any $P \in \mathbb{H}_{1}^{n+}$ and set $V=S-\mathcal{T}(S)$. It follows from (4.18) that

$$
\begin{aligned}
\langle\mathcal{L}(P) ; S\rangle-\langle P ; S\rangle & =\langle P ; \mathcal{T}(S)\rangle-\langle P ; S\rangle \\
& =\langle P ; S-V\rangle-\langle P ; S\rangle \\
& =\langle P ; S\rangle-\langle P ; V\rangle-\langle P ; S\rangle \\
& =-\langle P ; V\rangle,
\end{aligned}
$$


and that $\epsilon I \leq V(\ell) \mu$-almost everywhere in $\mathcal{S}$. From $S \in \mathbb{H}_{\text {sup }}^{n+}$ it follows that $S(\ell)<\beta I$ $\mu$-almost everywhere in $\mathcal{S}$, where $\|S\|_{\text {sup }}<\beta$. Combining these results and from (4.18) one gets, after noting that $\mathcal{T}(S)(\ell) \geq 0$, that

$$
\epsilon I \leq V(\ell) \leq V(\ell)+\mathcal{T}(S)(\ell)=S(\ell)<\beta I
$$

$\mu$-almost everywhere in $\mathcal{S}$. From (4.25) one gets that $\frac{\epsilon}{\beta}<1$, and that $\frac{\epsilon}{\beta} S(\ell)<\frac{\epsilon}{\beta} \beta I=$ $\epsilon I \leq V(\ell) \mu$-almost everywhere in $\mathcal{S}$. Therefore

$$
\begin{aligned}
\langle P ; V\rangle & =\int_{\mathcal{S}} \operatorname{tr}(P(\ell) V(\ell)) \mu(d \ell)=\int_{\mathcal{S}} \operatorname{tr}\left(P(\ell)^{1 / 2} V(\ell) P(\ell)^{1 / 2}\right) \mu(d \ell) \\
& \geq \frac{\epsilon}{\beta} \int_{\mathcal{S}} \operatorname{tr}\left(P(\ell)^{1 / 2} S(\ell) P(\ell)^{1 / 2}\right) \mu(d \ell) \\
& =\frac{\epsilon}{\beta} \int_{\mathcal{S}} \operatorname{tr}(P(\ell) S(\ell)) \mu(d \ell) \\
& =\frac{\epsilon}{\beta}\langle P ; S\rangle .
\end{aligned}
$$

Combining (4.24) and (4.26) it follows that $0 \leq\langle\mathcal{L}(P) ; S\rangle \leq\left(1-\frac{\epsilon}{\beta}\right)\langle P ; S\rangle$. Iterating this equation and making $P=R$, where $R$ is as in (4.5), it results that $0 \leq\left\langle\mathcal{L}^{k}(R) ; S\right\rangle \leq\left(1-\frac{\epsilon}{\beta}\right)^{k}\langle R ; S\rangle$. Notice from (4.13), (4.16), and (4.25) again, that $\left\langle\mathcal{L}^{k}(R) ; S\right\rangle=\int_{\mathcal{S}} \operatorname{tr}\left(\mathcal{L}^{k}(R)(\ell) S(\ell)\right) \mu(d \ell)=\int_{\mathcal{S}} \operatorname{tr}\left(\mathcal{L}^{k}(R)(\ell)^{1 / 2} S(\ell) \mathcal{L}^{k}(R)(\ell)^{1 / 2}\right) \mu(d \ell) \geq$ $\epsilon \operatorname{tr}\left(\int_{\mathcal{S}} \mathcal{L}^{k}(R)(\ell) \mu(d \ell)\right) \geq \epsilon \operatorname{tr}(Q(k))=\epsilon E\left(\|x(k)\|^{2}\right)$. Thus one can conclude that

$$
\sum_{k=0}^{\infty} E\left(\|x(k)\|^{2}\right) \leq \frac{1}{\epsilon}\langle R ; S\rangle \sum_{k=0}^{\infty}\left(1-\frac{\epsilon}{\beta}\right)^{k}=\frac{\beta}{\epsilon^{2}}\langle R ; S\rangle<\infty
$$

showing, according to Definition 4.1.2, that system (4.1) is SS.

iii) $\Longrightarrow i v)$ : In what follows set

$$
\widehat{\mathbb{H}}_{\text {sup }}^{n+}=\left\{P \in \mathbb{H}_{\text {sup }}^{n+} ; P(t) \geq \alpha I, \mu \text {-almost everywhere for } t \in \mathcal{S} \text {, for some } \alpha>0\right\}
$$

Recall that from Lemma 1 by Kubrusly $(1985), r_{\sigma}(\mathcal{L})<1$ implies that there exists $\gamma \geq 1$, $0<\rho<1$, such that $\left\|\mathcal{L}^{k}\right\| \leq \gamma \rho^{k}$ for every $k \in \mathbb{N}$. Consider any $V \in \widehat{\mathbb{H}}_{\text {sup }}^{n+}$. For any density $\nu$ with respect to $\mu$ so that $\int_{\mathcal{S}} \nu(\ell) \mu(d \ell)=1$, define $P^{\nu}$ as

$$
P^{\nu}(\ell)=I \nu(\ell)
$$


Then $P^{\nu} \in \mathbb{H}_{1}^{n+}$ and, for $\iota \in \mathbb{N}$,

$$
\begin{aligned}
0 & \leq\left\langle\sum_{k=0}^{\iota} \mathcal{L}^{k}\left(P^{\nu}\right) ; V\right\rangle=\left|\left\langle\sum_{k=0}^{\iota} \mathcal{L}^{k}\left(P^{\nu}\right) ; V\right\rangle\right|=\left|\sum_{k=0}^{\iota} \int_{\mathcal{S}} \operatorname{tr}\left(\mathcal{L}^{k}\left(P^{\nu}\right)(\ell) V(\ell)\right) \mu(d \ell)\right| \\
& \leq \sum_{k=0}^{\iota} \int_{\mathcal{S}}\left|\operatorname{tr}\left(\mathcal{L}^{k}\left(P^{\nu}\right)(\ell) V(\ell)\right)\right| \mu(d \ell) \\
& \leq n \sum_{k=0}^{\iota} \int_{\mathcal{S}}\left\|\mathcal{L}^{k}\left(P^{\nu}\right)(\ell)\right\|\|V(\ell)\| \mu(d \ell) \\
& \leq n\left(\sum_{k=0}^{\iota}\left\|\mathcal{L}^{k}\right\|\right)\|V\|_{\sup } \int_{\mathcal{S}}\left\|P^{\nu}(\ell)\right\| \mu(d \ell) \\
& \leq n \gamma\left(\sum_{k=0}^{\iota} \rho^{k}\right)\|V\|_{\text {sup }} \int_{\mathcal{S}} \nu(\ell) \mu(d \ell) \\
& \leq n \frac{\gamma}{1-\rho}\|V\|_{\text {sup }} .
\end{aligned}
$$

Define $S^{\iota} \in \widehat{\mathbb{H}}_{\text {sup }}^{n+}$, for $\iota \in \mathbb{N}$, as $S^{\iota}=\sum_{k=0}^{\iota} \mathcal{T}^{k}(V)$. It is easy to see that $S^{\iota+1}(\ell) \geq S^{\iota}(\ell) \geq V$ and that $\left\|S^{\iota}\right\|_{\text {sup }} \leq n\|V\|_{\text {sup }} \frac{\gamma}{1-\rho}$ for every $\iota \in \mathbb{N}$. Indeed, by contradiction, suppose that there exists $\iota \in \mathbb{N}$ and $C \in \mathcal{B}(\mathcal{S})$ with $\mu(C)>0$ such that $\left\|S^{\iota}(\ell)\right\|>n\|V\|_{\text {sup }} \frac{\gamma}{1-\rho}$ for all $\ell \in C$. Consider a density $\nu$ with respect to $\mu$ such that $\nu(\ell)=0$ for all $\ell \in C^{c}$, and $\int_{C} \nu(\ell) \mu(d \ell)=1$ (this density could be obtained as in Proposition 4.2.5, replacing $\mathcal{S}$ by $C$ ). Set $P^{\nu} \in \mathbb{H}_{1}^{n+}$ as in (4.27). From Proposition 4.2 .3 and (4.27) it follows that

$$
\begin{aligned}
\left\langle\sum_{k=0}^{\iota} \mathcal{L}^{k}\left(P^{\nu}\right) ; V\right\rangle & =\left\langle P^{\nu} ; \sum_{k=0}^{\iota} \mathcal{T}^{k}(V)\right\rangle=\left\langle P^{\nu} ; S^{\iota}\right\rangle=\int_{C} \operatorname{tr}\left(S^{\iota}(\ell)\right) \nu(\ell) \mu(d \ell) \\
& \geq \int_{C}\left\|S^{\iota}(\ell)\right\| \nu(\ell) \mu(d \ell) \\
& >n\|V\|_{\sup } \frac{\gamma}{1-\rho} \int_{C} \nu(\ell) \mu(d \ell)=n\|V\|_{\text {sup }} \frac{\gamma}{1-\rho},
\end{aligned}
$$

in contradiction with (4.28). Thus from the monotonicity result for positive semi-definite matrices (see Wonham, 1968, Lemma 3.1) it follows that there exists $S \in \widehat{\mathbb{H}}_{\text {sup }}^{n+}$ such that $S^{\iota}(\ell) \uparrow S(\ell)$ as $\iota \rightarrow \infty, \mu$-almost everywhere in $\mathcal{S}$, with $\|S\|_{\text {sup }} \leq n\|V\|_{\text {sup }} \frac{\gamma}{1-\rho}$. Moreover, by construction, $S^{\iota+1}=V+\mathcal{T}\left(S^{\iota}\right)$ and, from the Lebesgue convergence theorem (see Hernández-Lerma and Lasserre, 2003, Section 1.5.2),

$$
\lim _{\iota \rightarrow \infty} \int_{\mathcal{S}} S^{\iota}(s) g(\ell, s) \mu(d s)=\int_{\mathcal{S}} \lim _{\iota \rightarrow \infty} S^{\iota}(s) g(\ell, s) \mu(d s)=\int_{\mathcal{S}} S(s) g(\ell, s) \mu(d s) .
$$


Thus $\mu$-almost everywhere in $\mathcal{S}$ one gets that

$$
\begin{aligned}
S(\ell) & =\lim _{\iota \rightarrow \infty} S^{\iota+1}(\ell)=V(\ell)+\Gamma(\ell)^{*}\left(\lim _{\iota \rightarrow \infty} \int_{\mathcal{S}} S^{\iota}(s) g(\ell, s) \mu(d s)\right) \Gamma(\ell) \\
& =V(\ell)+\Gamma(\ell)^{*} \int_{\mathcal{S}} S(s) g(\ell, s) \mu(d s) \Gamma(\ell)=V(\ell)+\mathcal{T}(S)(\ell),
\end{aligned}
$$

so that (4.17) holds, completing the proof.

Some sufficient conditions for the existence of a solution for the "Lyapunov-like" (4.18) are presented next.

Corollary 4.3.4. If there exist $\{\delta(\ell) ; \ell \in \mathcal{S}\}$ and $\epsilon>0$ such that $\delta(\ell) \geq 0$, ess-sup $\{\delta(\ell) ; \ell \in \mathcal{S}\}<\infty$, and $\mu$-almost everywhere in $\mathcal{S}$

$$
\left\|\int_{\mathcal{S}} \delta(s) g(\ell, s) \mu(d s) \Gamma(\ell)^{*} \Gamma(\ell)\right\| \leq \delta(\ell)-\epsilon
$$

then system (4.1) is $S S$.

Proof. Set $S(\ell)=\delta(\ell) I$, so that $S \in \mathbb{H}_{\text {sup }}^{n+}$. From (4.8), one gets that

$$
\begin{aligned}
\mathcal{T}(S)(\ell) & =\Gamma(\ell)^{*}\left(\int_{\mathcal{S}} g(\ell, s) \delta(s) I \mu(d s)\right) \Gamma(\ell) \\
& =\int_{\mathcal{S}} \delta(s) g(\ell, s) \mu(d s) \Gamma(\ell)^{*} \Gamma(\ell) .
\end{aligned}
$$

Then, from (4.29) and (4.30) it follows $\mu$-almost everywhere in $\mathcal{S}$ that

$$
S(\ell)-\mathcal{T}(S)(\ell) \geq\left(\delta(\ell)-\left\|\int_{\mathcal{S}} \delta(s) g(\ell, s) \mu(d s) \Gamma(\ell)^{*} \Gamma(\ell)\right\|\right) I \geq \epsilon I
$$

so that (4.18) is satisfied, and the result follows from Theorem 4.3.3.

Corollary 4.3.5. If there exist $\{\delta(\ell) ; \ell \in \mathcal{S}\}$ and $\epsilon>0$ such that $\delta(\ell) \geq 0$, ess-sup $\{\delta(\ell) ; \ell \in \mathcal{S}\}<\infty$, and $\mu$-almost everywhere in $\mathcal{S}$

$$
\int_{\mathcal{S}} \delta(s) g(\ell, s) \mu(d s)\|\Gamma(\ell)\|^{2} \leq \delta(\ell)-\epsilon
$$

then system (4.1) is $S S$.

Proof. Since $\left\|\int_{\mathcal{S}} \delta(s) g(\ell, s) \mu(d s) \Gamma(\ell)^{*} \Gamma(\ell)\right\| \leq \int_{\mathcal{S}} \delta(s) g(\ell, s) \mu(d s)\|\Gamma(\ell)\|^{2}$, one can conclude that (4.31) implies (4.29), and the result follows from Corollary 4.3.4. 


\section{Chapter 5}

\section{LQ Optimal Control}

This chapter deals with the linear quadratic (LQ) optimal control for the case in which the the state variable $x(k)$ is observable. It is desired to design a state feedback control law so as to minimize a quadratic cost functional. Both the finite- and infinite-horizon optimal control problems are analyzed. The solutions for the optimal control problems are based on the Riccati operator $\mathcal{R}$, that is introduced in this chapter, which is related to a set of $\mathcal{S}$-coupled Riccati equations. By $\mathcal{S}$-coupled it is meant that the Riccati equations are coupled via an integral over the transition probability kernel $\mathcal{G}(\cdot, \cdot)$ having a density $g(\cdot, \cdot)$ with respect to the $\sigma$-finite measure $\mu$ on the Markov chain's state space $\mathcal{S}$. The solution for the finite-horizon LQ optimal control problem is derived in Section 5.1 from backward iterations of the Riccati operator $\mathcal{R}$. In Section 5.2 it is shown that the solution for the infinite-horizon LQ optimal control problem is obtained in terms of the stabilizing positive semi-definite solution $X(\ell), \ell \in \mathcal{S}$, for the control $\mathcal{S}$-coupled algebraic Riccati equations given by $X=\mathcal{R}(X)$.

\subsection{Finite-Horizon LQ Optimal Control}

In this section the finite-horizon LQ optimal control problem is tackled. With this goal in mind, it is defined the Riccati operator $\mathcal{R}$ and some auxiliary operators $\mathcal{Q}$ and $\mathcal{K}$, the latter related to the feedback gain for the optimal control. In Theorem 5.1.6 the optimal controller is derived from backward iterations of the Riccati operator $\mathcal{R}$. 


\subsubsection{Problem Statement}

On the filtered probability space $\left(\Omega, \mathcal{F},\left\{\mathcal{F}_{k}\right\}_{k \in \mathbb{N}}, \mathcal{P}\right)$ consider the following discrete-time Markov jump linear system

$$
x(k+1)=A(\theta(k)) x(k)+B(\theta(k)) u(k) .
$$

Recall that the assumptions stated in Section 3.3 hold, in particular Assumption 3.2.2 holds and $E\left(\left\|x_{0}\right\|^{2}\right)<\infty$. Assume also the hypothesis stated below that requires the system's parameters to be bounded (in the ess-sup sense).

Assumption 5.1.1. $A \in \widetilde{\mathbb{H}}_{\text {sup }}^{n}, B \in \widetilde{\mathbb{H}}_{\text {sup }}^{m, n}$.

In this section consider a fixed positive $\tau \in \mathbb{N}, \tau>1$, that represents the control problem's time horizon. The set of nonnegative integers less or equal to $\tau-1$ is denoted by $\mathbb{T}$. Consider also $S \in \widetilde{\mathbb{H}}_{\text {sup }}^{n+}$, and set $\mathcal{U}^{\tau}$ as the class of admissible controls as defined next.

Definition 5.1.2. - Admissible Controls $\mathcal{U}^{\tau}$ : The class of admissible controls with finite-time horizon $\tau$ is defined as the class of controls $u=\{u(k), k \in \mathbb{T}\}$ such that $u(k)$ is $\mathcal{F}_{k}$-measurable, for all $k \in \mathbb{T}$.

The finite-horizon LQ optimal control problem that is considered in this section is defined below.

Problem 5.1.3. - Finite-Horizon LQ Optimal Control Problem: Find $u \in \mathcal{U}^{\tau}$ that minimizes the $L Q$ cost $J_{S}^{\tau}\left(x_{0}, \theta_{0}, u\right)$ given by

$$
J_{S}^{\tau}\left(x_{0}, \theta_{0}, u\right):=E\left(\sum_{k=0}^{\tau-1}\|C(\theta(k)) x(k)\|^{2}+\|D(\theta(k)) u(k)\|^{2}+x(\tau)^{*} S(\theta(\tau)) x(\tau)\right)
$$

where $C \in \widetilde{\mathbb{H}}_{\text {sup }}^{n, r}, D \in \widetilde{\mathbb{H}}_{\text {sup }}^{m, p}$ and $D(\ell)^{*} D(\ell) \geq \epsilon_{D} I$ u-almost everywhere in $\mathcal{S}$ for some $0<\epsilon_{D} \leq 1$. The finite-horizon optimal $L Q$ cost is defined as

$$
J_{S}^{\tau}\left(x_{0}, \theta_{0}\right):=\inf _{u \in \mathcal{U}^{\tau}} J_{S}^{\tau}\left(x_{0}, \theta_{0}, u\right)
$$

\subsubsection{The Optimal Control Law}

Recall the definition of operator $\mathcal{E}$ stated in (4.6). The operators necessary to address the Problem 5.1.3 are defined next. 
Definition 5.1.4. For $Z \in \mathbb{H}_{\text {sup }}^{n+}$ define the operators $\mathcal{Q}, \mathcal{K}$, and $\mathcal{R}$, for $\ell \in \mathcal{S}$, as follows,

$$
\begin{aligned}
& \mathcal{Q}(Z)(\ell):= D(\ell)^{*} D(\ell)+B(\ell)^{*} \mathcal{E}(Z)(\ell) B(\ell), \\
& \mathcal{K}(Z)(\ell):=\mathcal{Q}(Z)(\ell)^{-1} B(\ell)^{*} \mathcal{E}(Z)(\ell) A(\ell), \\
& \mathcal{R}(Z)(\ell):=C(\ell)^{*} C(\ell)+A(\ell)^{*} \mathcal{E}(Z)(\ell) A(\ell) \\
& \quad-A(\ell)^{*} \mathcal{E}(Z)(\ell) B(\ell) \mathcal{Q}(Z)(\ell)^{-1} B(\ell)^{*} \mathcal{E}(Z)(\ell) A(\ell) .
\end{aligned}
$$

Proposition 5.1.5. $\mathcal{E}, \mathcal{Q}, \mathcal{K}$ and $\mathcal{R}$ map $\mathbb{H}_{\text {sup }}^{n+}$ into respectively $\mathbb{H}_{\text {sup }}^{n+}, \mathbb{H}_{\text {sup }}^{m+}, \mathbb{H}_{\text {sup }}^{n, m}$ and $\mathbb{H}_{\text {sup }}^{n+}$, and $\mathcal{Q}(Z)^{-1} \in \mathbb{H}_{\text {sup }}^{m+}$ for $Z \in \mathbb{H}_{\text {sup }}^{n+}$.

Proof. It follows similar arguments as in Proposition 3 in (Costa and Fragoso, 1995).

For $k=\tau-1, \ldots, 0$, define the control $\mathcal{S}$-coupled Riccati difference equations

$$
\begin{aligned}
& X^{\tau}(k)=\mathcal{R}\left(X^{\tau}(k+1)\right) \\
& X^{\tau}(\tau)=S
\end{aligned}
$$

where from $S \in \widetilde{\mathbb{H}}_{\text {sup }}^{n+}$ and Proposition 5.1.5 it follows that $X(k) \in \mathbb{H}_{\text {sup }}^{n+}$, and define also the feedback gains $K(k) \in \mathbb{H}_{\text {sup }}^{n, m}$ as

$$
K^{\tau}(k)=-\mathcal{K}\left(X^{\tau}(k+1)\right),
$$

and

$$
V^{\tau}(k)=\mathcal{Q}\left(X^{\tau}(k+1)\right)
$$

Recall that, as stated in Remark 3.1.5, $X^{\tau}(k)$ means $X^{\tau}(k, \ell)$, for $\ell \in \mathcal{S}$, and it also holds for the iterative mappings $K^{\tau}(k)$ and $V^{\tau}(k)$.

The next result can be derived following the same arguments, mutatis mutandi, as those applied to solve the finite-horizon LQ optimal control problem for MJLS with Markov chain in a countably infinite state space (see Costa and Fragoso, 1995). That is, by applying the Bellman's principle of optimality the solution for the finite-horizon LQ optimal control problem stated in Problem 5.1.3 is obtained from the backward Riccati iterations defined in the control $\mathcal{S}$-coupled Riccati difference equations (5.4).

Theorem 5.1.6. It follows that

$$
J_{S}^{\tau}\left(x_{0}, \theta_{0}, u\right)=E\left(\sum_{k=0}^{\tau-1} \| B(\theta(k))^{*} \mathcal{E}\left(X^{\tau}(k+1)\right)(\theta(k)) A(\theta(k)) x(k)\right.
$$




$$
\left.+V^{\tau}(k, \theta(k)) u(k) \|_{V^{\tau}(k, \theta(k))}^{2}\right)+E\left(x_{0}^{*} X^{\tau}\left(0, \theta_{0}\right) x_{0}\right)
$$

Moreover,

$$
J_{S}^{\tau}\left(x_{0}, \theta_{0}\right)=E\left(x_{0}^{*} X^{\tau}\left(0, \theta_{0}\right) x_{0}\right)=J_{S}^{\tau}\left(x_{0}, \theta_{0}, \hat{u}^{\tau}\right),
$$

where $\hat{u}^{\tau}(k)=K^{\tau}(k, \theta(k)) x(k)$.

Proof. See Propositions 4 and 5 in (Costa and Fragoso, 1995).

\subsection{Infinite-Horizon LQ Optimal Control}

The optimal control law stated in Theorem 5.1.6 provides a controller with time-varying gains. From the point of view of implementation, it would be interesting to simplify this solution by using a stationary controller (Mariton, 1990, Section 3.2.3). With this goal in mind, in this section the infinite-horizon LQ optimal control problem is tackled. It is shown that the optimal control law is in a time invariant (stationary) state feedback form, and is obtained in terms of the stabilizing positive semi-definite solution of the control $\mathcal{S}$-coupled algebraic Riccati equations. Sufficient conditions for the existence and uniqueness of this stabilizing positive semi-definite solution for the control $\mathcal{S}$-coupled algebraic Riccati equations, based on the concepts of (stochastic) stabilizability and detectability, are presented in Appendix A.1. In Theorem A.1.6 it is shown that under the assumption of $(A, B)$ being stochastically stabilizable (that is, there must exist a control that stochastically stabilizes the system), there exists a solution $X(\ell), \ell \in \mathcal{S}$, for the control $\mathcal{S}$-coupled algebraic Riccati equations. If in addition $(C, A)$ is stochastically detectable (that is, the unstable part of the state trajectory must be reflected in the cost functional) then $X$ is unique (in the $\mu$-almost everywhere sense) among the positive semi-definite solutions, it is stabilizing, and from Theorem 5.2.5 it provides a solution for the infinite-horizon LQ optimal control problem.

\subsubsection{Problem Statement}

On the filtered probability space $\left(\Omega, \mathcal{F},\left\{\mathcal{F}_{k}\right\}_{k \in \mathbb{N}}, \mathcal{P}\right)$ consider the discrete-time MJLS (5.1). In this section assume the class of admissible controls as follows.

Definition 5.2.1. - Admissible Controls $\mathcal{U}$ : The class of admissible controls $\mathcal{U}$ is defined as the class of controls $u=\{u(k), k \in \mathbb{N}\}$ such that $u(k)$ is $\mathcal{F}_{k}$-measurable, for all 
$k \in \mathbb{N}$, and the system (5.1) is mean square stable in the sense that $E\left(\|x(k)\|^{2}\right) \rightarrow 0$ as $k \rightarrow \infty$ for any initial distribution $\widehat{\mu}$ satisfying Assumption 3.2.2 and $E\left(\left\|x_{0}\right\|^{2}\right)<\infty$.

The infinite-horizon LQ optimal control problem that is considered in this section is stated below.

Problem 5.2.2. - Infinite-Horizon LQ Optimal Control Problem: Find $u \in \mathcal{U}$ that minimizes the $L Q$ cost $J\left(x_{0}, \theta_{0}, u\right)$ given by

$$
J\left(x_{0}, \theta_{0}, u\right):=E\left(\sum_{k=0}^{\infty}\|C(\theta(k)) x(k)\|^{2}+\|D(\theta(k)) u(k)\|^{2}\right),
$$

where $C \in \widetilde{\mathbb{H}}_{\text {sup }}^{n, r}, D \in \widetilde{\mathbb{H}}_{\text {sup }}^{m, p}$, and $D(\ell)^{*} D(\ell) \geq \epsilon_{D} I$-almost everywhere in $\mathcal{S}$ for some $0<\epsilon_{D} \leq 1$. The optimal $L Q$ cost is defined as

$$
J\left(x_{0}, \theta_{0}\right):=\inf _{u \in \mathcal{U}} J\left(x_{0}, \theta_{0}, u\right)
$$

The definitions of (stochastic) stabilizability and detectability that are used in what follows are stated below. These definitions follow from standard concepts of stabilizability and detectability in the control theory literature, adapted to the present context (see, for instance, Theorems 62 ii) and 65 ii) in (Callier and Desoer, 1991, Chapter 8)).

Definition 5.2.3. - Stochastic Stabilizability: For $F \in \mathbb{H}_{\text {sup }}^{r, n},(A, F)$ is said stochastically stabilizable if $r_{\sigma}(\mathcal{L})<1$ for some $J \in \mathbb{H}_{\text {sup }}^{n, r}$, where $\mathcal{L}$ is as in (4.7) above with $\Gamma(\ell)=A(\ell)+F(\ell) J(\ell), \ell \in \mathcal{S}$. In this case, it is said that $J$ stochastically stabilizes $(A, F)$.

Definition 5.2.4. - Stochastic Detectability: For $F \in \mathbb{H}_{\text {sup }}^{n, r},(F, A)$ is said stochastically detectable if $r_{\sigma}(\mathcal{L})<1$ for some $J \in \mathbb{H}_{\text {sup }}^{r, n}$, where $\mathcal{L}$ is as in (4.7) above with $\Gamma(\ell)=A(\ell)+J(\ell) F(\ell), \ell \in \mathcal{S}$. In this case, it is said that $J$ stochastically stabilizes $(F, A)$.

\subsubsection{The Optimal Control Law}

The solution of the infinite-horizon optimal control problem stated in Problem 5.2.2 is related to the existence of stabilizing solutions $X \in \widetilde{\mathbb{H}}_{\text {sup }}^{n+}$ for the following control $\mathcal{S}$ coupled algebraic Riccati equations,

$$
X(\ell)=\mathcal{R}(X)(\ell) \quad \mu \text {-almost everywhere in } \mathcal{S} \text {. }
$$


A solution $X \in \widetilde{\mathbb{H}}_{\text {sup }}^{n+}$ for the control $\mathcal{S}$-coupled algebraic Riccati equations is called stabilizing if $K=-\mathcal{K}(X)$ stochastically stabilizes $(A, B)$ (see Definition A.1.3).

The conditions for the existence of stabilizing positive semi-definite solutions for the control $\mathcal{S}$-coupled algebraic Riccati equations (5.9) are presented in Appendix A.1. Proposition A.1.4 provides a sufficient condition, based on the concept of stochastic stabilizability, for the existence of a positive semi-definite solution and $\mu$-almost everywhere convergence of iterations of the operator $\mathcal{R}$ starting from zero to this solution, while Proposition A.1.5 provides a sufficient condition, based on the concept of stochastic detectability, to guarantee that this is in fact a stabilizing solution. Theorem A.1.6 combines these results to guarantee the existence and uniqueness among the positive semi-definite solutions for the control $\mathcal{S}$-coupled algebraic Riccati equations. Furthermore it shows that this solution is the stabilizing one and the iterations of the operator $\mathcal{R}$ starting from any positive semi-definite element will converge $\mu$-almost everywhere to this solution. From these results, the next theorem provides the solution to the infinite-horizon LQ optimal control problem stated in Problem 5.2.2.

Theorem 5.2.5. Suppose that $(A, B)$ is stochastically stabilizable and $(C, A)$ is stochastically detectable. Then there exists a unique stabilizing solution $X$ for the control $\mathcal{S}$-coupled algebraic Riccati equations (5.9) and setting $K=-\mathcal{K}(X)$ it follows, for any initial distribution $\widehat{\mu}$ satisfying $E\left(\left\|x_{0}\right\|^{2}\right)<\infty$ and Assumption 3.2.2, that

$$
J\left(x_{0}, \theta_{0}\right)=E\left(x_{0}^{*} X\left(\theta_{0}\right) x_{0}\right)=J\left(x_{0}, \theta_{0}, \hat{u}\right),
$$

where $\hat{u}(k)=K(\theta(k)) x(k)$.

Proof. The existence of a unique stabilizing positive semi-definite solution $X \in \widetilde{\mathbb{H}}_{\text {sup }}^{n+}$ for the control $\mathcal{S}$-coupled algebraic Riccati equations (5.9) follows from Theorem A.1.6. Considering $S=X$ in (5.4) one gets that $X^{\tau}(0)=X$ and $V^{\tau}(k)=V=\mathcal{Q}(X)$ for all $\tau$ due to (5.9). From (5.2) and taking the limit as $\tau \rightarrow \infty$ in Theorem 5.1.6 one gets that for any $u \in \mathcal{U}$,

$$
\begin{aligned}
J\left(x_{0}, \theta_{0}, u\right)=E\left(\sum_{k=0}^{\infty}\|C(\theta(k)) x(k)\|^{2}+\|D(\theta(k)) u(k)\|^{2}\right) \\
=E\left(\sum_{k=0}^{\infty} \| B(\theta(k))^{*} \mathcal{E}\left(X(\theta(k)) A(\theta(k)) x(k)+V(\theta(k)) u(k) \|_{V(\theta(k))}^{2}\right)\right. \\
\quad+E\left(x_{0}^{*} X\left(\theta_{0}\right) x_{0}\right),
\end{aligned}
$$


since $u \in \mathcal{U}$ (see Definition 5.2.1) one gets that

$$
0 \leq E\left(x(\tau)^{*} X(\theta(\tau)) x(\tau)\right) \leq\|X\|_{\text {sup }} E\left(\|x(\tau)\|^{2}\right) \rightarrow 0 \quad \text { as } \quad \tau \rightarrow \infty
$$

and thus $E\left(x(\tau)^{*} X(\theta(\tau)) x(\tau)\right)=0$. Choosing, for $k \in \mathbb{N}$,

$$
\begin{aligned}
u(k) & =\widehat{u}(k)=K(\theta(k)) x(k) \\
& =-\mathcal{K}(X)(\theta(k)) x(k) \\
& =-\mathcal{Q}(X)(\theta(k))^{-1} B(\theta(k))^{*} \mathcal{E}(X)(\theta(k)) A(\theta(k)) \\
& =-V(\theta(k))^{-1} B(\theta(k))^{*} \mathcal{E}(X)(\theta(k)) A(\theta(k))
\end{aligned}
$$

in (5.10) it follows that $J\left(x_{0}, \theta_{0}\right)=E\left(x_{0}^{*} X\left(\theta_{0}\right) x_{0}\right)=J\left(x_{0}, \theta_{0}, \widehat{u}\right)$. 


\section{Chapter 6}

\section{Filtering}

This chapter considers the design of minimum mean square linear Markov jump filters. It is assumed that a measurable output $y(k)$ and the jump parameter $\theta(k)$ are known at each discrete-time instant $k$. The optimal state estimator for the case in which an output and the jump parameter of the system are available is a Kalman filter (KF) for a time varying system, and therefore the filter gains are sample path dependent (see Davis and Vinter, 1985), making any stability and infinite-horizon analysis much harder. Moreover, as discussed by Auger et al. (2013), digital real-time implementation of the KF has always been a challenging issue due to roundoff errors when computing the covariance matrices, and computational load and execution time of the KF. To avoid this sample path dependence and to allow an offline calculation of the filter, as in (Costa, 1995; Costa and Tuesta, 2003), only the class of Markov jump filters (that is, filters that depend just on the present values of the Markov parameter and measurable output) is considered. The finite and the infinite-horizon filtering problems are addressed in Sections 6.1 and 6.2, respectively. In the filtering problems it is desired to minimize a quadratic cost among the class of Markov jump filters. In Section 6.1 it is shown that an optimal filter for the finite-horizon filtering problem is obtained from recursive $\mathcal{S}$-coupled Riccati equations, named filtering $\mathcal{S}$-coupled Riccati difference equations. A solution for the infinite-horizon filtering problem is obtained in Section 6.2 in terms of the stabilizing positive semi-definite solution to the filtering $\mathcal{S}$-coupled algebraic Riccati equations.

\subsection{Finite-Horizon Filtering}

In this section the finite-horizon filtering problem is considered. Theorem 6.1.21 presents the optimal filter, named finite-horizon minimum mean square linear Markov jump filter, 
which is obtained from the filtering $\mathcal{S}$-coupled Riccati difference equations (6.21).

\subsubsection{Problem Statement}

On the filtered probability space $\left(\Omega, \mathcal{F},\left\{\mathcal{F}_{k}\right\}_{k \in \mathbb{N}}, \mathcal{P}\right)$ consider the following discrete-time Markov jump linear system

$$
\left\{\begin{aligned}
x(k+1) & =A(\theta(k)) x(k)+B(\theta(k)) u(k)+G(\theta(k)) w(k) \\
y(k) & =L(\theta(k)) x(k)+H(\theta(k)) w(k) .
\end{aligned}\right.
$$

Recall that the assumptions stated in Section 3.3 hold, in particular $E\left(x_{0}\right):=\zeta$, $E\left(x_{0} x_{0}^{*}\right):=\mathbb{Q}_{0}$, and thus $E\left(\left\|x_{0}-\zeta\right\|^{2}\right)=\operatorname{tr}\left(\mathbb{Q}_{0}-\zeta \zeta^{*}\right)<\infty$. As stated in Remark 3.2.3, in this section the Assumption 3.2.2 is not considered and it is only assumed the existence of $\widehat{\mu}_{\mathcal{S}}$, the initial probability measure on $\mathcal{S}$ for $\theta_{0}$.

Remark 6.1.1. Throughout this section, for notational simplicity, it is assumed that the Markov chain is time-homogeneous and as well as the systems parameters $A, B$, etc. But this assumption is not essential as all the results could be easily adapted to the time-varying case.

Define the sequence $\{\pi(k) ; k \in \mathbb{N}\}$ as

$$
\begin{aligned}
\pi(k+1, \ell) & :=E(g(\theta(k), \ell)), \quad \text { for } k \in \mathbb{N}, \\
\pi(0, \ell) & :=1 .
\end{aligned}
$$

Proposition 6.1.2. The following recursive expression for $\pi(k), k \in \mathbb{N}_{1}$ holds,

$$
\pi(k+1, \ell)=\int_{\mathcal{S}} g(s, \ell) \pi(k, s) \mu(d s)
$$

Proof. The result will be shown by induction on $k$. Recalling that $\pi(1, \ell)=E\left(g\left(\theta_{0}, \ell\right)\right)=$ $\int_{\mathcal{S}} g(s, \ell) \widehat{\mu}_{\mathcal{S}}(d s)$ one gets from Fubini's theorem, (3.11) and (6.2) with $k=1$ that

$$
\begin{aligned}
\pi(2, \ell) & =E(g(\theta(1), \ell))=\int_{\mathcal{S}} \int_{\mathcal{S}} g(s, \ell) g(r, s) \mu(d s) \widehat{\mu}_{\mathcal{S}}(d r) \\
& =\int_{\mathcal{S}} g(s, \ell) \int_{\mathcal{S}} g(r, s) \widehat{\mu}_{\mathcal{S}}(d r) \mu(d s) \\
& =\int_{\mathcal{S}} g(s, \ell) \pi(1, s) \mu(d s) .
\end{aligned}
$$


Suppose the result holds for $k>1$. Then, from Fubini's theorem again, (3.11) and (6.2), it follows that

$$
\begin{aligned}
\pi(k+1, \ell) & =E(g(\theta(k), \ell))=E(E(g(\theta(k), \ell) \mid \theta(k-1))) \\
& =E\left(\int_{\mathcal{S}} g(s, \ell) g(\theta(k-1), s) \mu(d s)\right) \\
& =\int_{\mathcal{S}} g(s, \ell) E(g(\theta(k-1), s)) \mu(d s) \\
& =\int_{\mathcal{S}} g(s, \ell) \pi(k, s) \mu(d s) .
\end{aligned}
$$

The next proposition states that $\pi(k)$ is the probability density function of $\theta(k)$ with respect to $\mu$ for $k \in \mathbb{N}_{1}$.

Proposition 6.1.3. For $k \in \mathbb{N}$ and for $B \in \mathcal{B}(\mathcal{S})$,

$$
\mathcal{P}(\theta(k+1) \in B)=\int_{B} \pi(k+1, \ell) \mu(d \ell)
$$

Proof. From Fubini's theorem and (6.2) one gets that for any $B \in \mathcal{B}(\mathcal{S})$ and $k \in \mathbb{N}$,

$$
\begin{aligned}
\mathcal{P}(\theta(k+1) \in B) & =E(\mathcal{P}(\theta(k+1) \in B \mid \theta(k)))=E\left(\int_{B} g(\theta(k), \ell) \mu(d \ell)\right) \\
& =\int_{B} E(g(\theta(k), \ell)) \mu(d \ell)=\int_{B} \pi(k+1, \ell) \mu(d \ell) .
\end{aligned}
$$

Thus, for $k \in \mathbb{N}, \pi(k+1)$ is the density function of $\theta(k+1)$ with respect to $\mu$.

In this section assume also the hypotheses stated below.

Assumption 6.1.4. The noise sequence $\{w(k) ; k \in \mathbb{N}\}$ is a q-dimensional zero mean white noise sequence (that is, $\{w(k) ; k \in \mathbb{N}\}$ is a sequence of independent identically distributed random vectors with $E(w(k))=0$ and $\left.E\left(w(k) w(k)^{\prime}\right)=I\right)$, and is assumed also that $\{w(k) ; k \in \mathbb{N}\}$, the Markov chain $\{\theta(k) ; k \in \mathbb{N}\}$, and the initial state $x_{0}$ are mutually independent.

Assumption 6.1.5. $A \in \widetilde{\mathbb{H}}_{\text {sup }}^{n}, B \in \widetilde{\mathbb{H}}_{\text {sup }}^{m, n}, G \in \widetilde{\mathbb{H}}_{\text {sup }}^{q, n}, L \in \widetilde{\mathbb{H}}_{\text {sup }}^{n, p}, H \in \widehat{\mathbb{H}}_{\text {sup }}^{q, p}$.

Assumption 6.1.6. $G(\ell) H(\ell)^{*}=0$ for all $\ell \in \mathcal{S}$.

Assumption 6.1.7. For some $c \geq 1$ it holds that $g(t, \ell) \leq c$ for all $t, \ell \in \mathcal{S}$. 
Assumption 6.1.8. There exist $d>0$ and $\epsilon_{H}(\ell)>0$ such that, for each $k \in \mathbb{N}$, $H(\ell) H(\ell)^{*} \pi(k, \ell) \geq \epsilon_{H}(\ell) I$ and $\frac{\|A(\ell)\|\|L(\ell)\|}{\epsilon_{H}(\ell)} \leq d$ for all $\ell \in \mathcal{S}$.

It is worth pointing out some remarks regarding Assumptions 6.1.4 to 6.1.8. Assumption 6.1.4 is usual in the LQG literature for the noise input sequence, as well as the independence condition between the noise, the initial state, and the Markov chain when considering Markov jump systems. Assumption 6.1.5 requires that the parameters of the system (6.1) to be bounded (in the ess-sup sense). Regarding Assumption 6.1.6, in most treatment of the LQG literature it is considered that the state and observation noises are different and independent, that is, it is assumed that the state noise is given by $\widetilde{G}(\theta(k)) w^{x}(k)$ and the observation noise by $\widetilde{H}(\theta(k)) w^{y}(k)$. By defining,

$$
w(k)=\left[\begin{array}{l}
w^{x}(k) \\
w^{y}(k)
\end{array}\right], \quad G(\theta(k))=\left[\begin{array}{ll}
\widetilde{G}(\theta(k)) & 0
\end{array}\right], \quad H(\theta(k))=\left[\begin{array}{ll}
0 & \widetilde{H}(\theta(k))
\end{array}\right],
$$

the corresponding terms in (6.1) follow and clearly Assumption 6.1.6 is satisfied. In any case, as pointed out in Remark 5.1 in (Costa et al., 2005c, p. 103), Assumption 6.1.6 represents no loss of generality since it could be removed by making some appropriate changes in the system equations (6.1). Most of the transition density functions found in applications would satisfy the uniform boundedness condition required in Assumption 6.1.7. As usual in the LQG literature, Assumption 6.1.8 imposes a positive condition on the covariance matrices of the observation noises, weighted in the Markov jump case by the probability density function of $\theta(k)$ with respect to $\mu$, represented by $\pi(k)$ for $k \in \mathbb{N}_{1}$, so that the inverse of some matrices can be assured (see Remark 6.1.16 below). The condition $\frac{\|A(\ell)\|\|L(\ell)\|}{\epsilon_{H}(\ell)} \leq d$ is required to obtain the boundedness of an operator (see (6.11) below).

Proposition 6.1.9. It holds that $\pi(k) \in \mathbb{H}_{1}^{1+} \cap \mathbb{H}_{\text {sup }}^{1+}$ for $k \in \mathbb{N}_{1}$. Moreover, $\|\pi(k)\|_{1}=1$.

Proof. The result will be shown by induction on $k$. For $k=1$ the result is immediate since one gets from Fubini's theorem and (6.2) that

$$
\begin{aligned}
\|\pi(1)\|_{1} & =\int_{\mathcal{S}} \int_{\mathcal{S}} g(s, \ell) \widehat{\mu}_{\mathcal{S}}(d s) \mu(d \ell)=\int_{\mathcal{S}} \int_{\mathcal{S}} g(s, \ell) \mu(d \ell) \widehat{\mu}_{\mathcal{S}}(d s) \\
& =\int_{\mathcal{S}} \widehat{\mu}_{\mathcal{S}}(d s)=1
\end{aligned}
$$


after recalling that $\int_{\mathcal{S}} g(s, \ell) \mu(d \ell)=1$, and since that, from Assumption 6.1.7, for $\ell \in \mathcal{S}$,

$$
\|\pi(1, \ell)\|=\left\|\int_{\mathcal{S}} g(s, \ell) \widehat{\mu}_{\mathcal{S}}(d s)\right\| \leq c\left\|\int_{\mathcal{S}} \widehat{\mu}_{\mathcal{S}}(d s)\right\|=c
$$

Suppose the result holds for $k>1$. Then from Fubini's theorem and (6.3) it follows that

$$
\begin{aligned}
\|\pi(k+1)\|_{1} & =\int_{\mathcal{S}} \int_{\mathcal{S}} \pi(k, s) g(s, \ell) \mu(d s) \mu(d \ell)=\int_{\mathcal{S}} \pi(k, s) \int_{\mathcal{S}} g(s, \ell) \mu(d \ell) \mu(d s) \\
& =\int_{\mathcal{S}} \pi(k, s) \mu(d s)=\|\pi(k)\|_{1}=1,
\end{aligned}
$$

since again $\int_{\mathcal{S}} g(s, \ell) \mu(d \ell)=1$, and from Assumption 6.1.7 and (6.3) one gets that

$$
\begin{aligned}
\|\pi(k+1, \ell)\| & =\left\|\int_{\mathcal{S}} \pi(k, s) g(s, \ell) \mu(d s)\right\| \\
& \leq c\left\|\int_{\mathcal{S}} \pi(k, s) \mu(d s)\right\|=c\|\pi(k)\|_{1}=c .
\end{aligned}
$$

Then indeed $\pi(k) \in \mathbb{H}_{1}^{1+} \cap \mathbb{H}_{\text {sup }}^{1+}$ and $\|\pi(k)\|_{1}=1$, for $k \in \mathbb{N}_{1}$, completing the proof.

Consider a fixed positive $\tau \in \mathbb{N}, \tau>1$, that represents the filtering problem's time horizon. Recall that the set of nonnegative integers less or equal to $\tau-1$ is denoted by $\mathbb{T}$ and the set of positive integers less or equal to $\tau-1$ is denoted by $\mathbb{T}_{1}$.

The class of admissible filters that is considered in this section is defined next. It is assumed that the measurable output $y(k)$ and the jump parameter $\theta(k)$ are known at each discrete-time instant $k$.

Definition 6.1.10. - Admissible Filters $\mathcal{F}^{\tau}$ : The class of linear Markov jump filters $\mathcal{F}^{\tau}$ is given by

$$
\left\{\begin{aligned}
\widehat{x}(k+1) & =\widehat{A}(k, \theta(k)) \widehat{x}(k)+\widehat{B}(k, \theta(k)) y(k) \\
u(k) & =\widehat{C}(k, \theta(k)) \widehat{x}(k),
\end{aligned}\right.
$$

where $\widehat{x}(0)=\widehat{x}_{0} \in \mathbb{R}^{n}$ deterministic and $\widehat{A}(k) \in \widetilde{\mathbb{H}}_{\text {sup }}^{n}, \widehat{B}(k) \in \widetilde{\mathbb{H}}_{\text {sup }}^{p, n}$ and $\widehat{C}(k) \in \widetilde{\mathbb{H}}_{\text {sup }}^{n, m}$, for each $k \in \mathbb{T}$.

Following similar arguments as those presented in Proposition 4.3.2, it follows from the Assumption 6.1 .5 and that $\widehat{A}(k) \in \widetilde{\mathbb{H}}_{\text {sup }}^{n}, \widehat{B}(k) \in \widetilde{\mathbb{H}}_{\text {sup }}^{p, n}$ and $\widehat{C}(k) \in \widetilde{\mathbb{H}}_{\text {sup }}^{n, m}$, that $E\left(\|x(k)\|^{2}\right)<\infty$ and $E\left(\|\widehat{x}(k)\|^{2}\right)<\infty, k \in \mathbb{T}$.

Notice that $u(k)$ in (6.5) is the control variable of the system (6.1). As it will be shown in this section and in the next, the optimal filters do not depend on the control variable. 
However, in Chapter 7 the partial information optimal control problem is analyzed and in this case it is useful to consider the presence of the control variable in the filtering problem.

Remark 6.1.11. Recall that the $\sigma$-field generated by $\left\{x_{0}, \theta_{0}, w(0), \theta(1), w(1), \ldots, \theta(k)\right.$, $w(k)\}, k \in \mathbb{N}$, is denoted by $\mathcal{F}_{k}$, and the set of positive integers is denoted by $\mathbb{N}_{1}$. For any $u$ as in (6.5) it follows that $x(k)$ and $\widehat{x}(k)$ are $\mathcal{F}_{k-1}$-measurable for $k \in \mathbb{N}_{1}$. Indeed it is easy to see from (6.1) and (6.5) that $x(k)$ and $\widehat{x}(k)$ can be written in function of $x_{0}$ and $w(j), \theta(j)$, for $j=0, \ldots, k-1$. Moreover, by definition one has that $x_{0}$ is $\mathcal{F}_{0}$-measurable and $\widehat{x}(0)=\widehat{x}_{0}$ is deterministic.

Formally the optimal finite-horizon filtering problem, with time horizon $\tau$, that is considered in this section is defined below.

Problem 6.1.12. - Finite-Horizon Filtering Problem: Find $\widehat{A}(k) \in \mathbb{H}_{\text {sup }}^{n}, \widehat{B}(k) \in$ $\mathbb{H}_{\text {sup }}^{p, n}$ and $\widehat{C}(k) \in \mathbb{H}_{\text {sup }}^{n, m}, k \in \mathbb{T}$, and $\widehat{x}_{0} \in \mathbb{R}^{n}$ in (6.5) that minimizes the cost

$$
\sum_{k=0}^{\tau} E\left(\|v(k)\|^{2}\right)
$$

where

$$
v(k):=x(k)-\widehat{x}(k) .
$$

\subsubsection{Finite-Horizon Optimal Filter}

Next, some operators necessary to address the Problem 6.1.12 are defined. For $V \in$ $\mathbb{H}_{1}^{n} \cap \mathbb{H}_{\text {sup }}^{n}, U \in \mathbb{H}_{\text {sup }}^{n} K \in \mathbb{H}_{\text {sup }}^{p, n}, \alpha \in \mathbb{H}_{1}^{1+} \cap \mathbb{H}_{\text {sup }}^{1+}, \beta \in \mathbb{H}_{1}^{1+}$, and $k \in \mathbb{N}$, define the operators $\mathcal{L}(K, V), \mathcal{I}(K, V, \alpha), \mathcal{U}(K, V, \alpha), \mathcal{U}(V, \alpha)$, and $\mathcal{M}(U, \beta)$, such that

$$
\begin{aligned}
\mathcal{L}(K, V)(\ell) & :=\int_{\mathcal{S}} g(s, \ell)(A(s)+K(s) L(s)) V(s)(A(s)+K(s) L(s))^{*} \mu(d s) \\
\mathcal{I}(K, V, \alpha)(\ell) & :=\int_{\mathcal{S}} g(s, \ell)\left(\alpha(s)\left((K(s) H(s))(K(s) H(s))^{*}+G(s) G(s)^{*}\right)+V(s)\right) \mu(d s) \\
\mathcal{U}(K, V, \alpha)(\ell) & :=\mathcal{L}(K, V)(\ell)+\mathcal{I}(K, 0, \alpha)(\ell) \\
\mathcal{M}(U, \beta)(\ell) & :=-A(\ell) U(\ell) L(\ell)^{*}\left(L(\ell) U(\ell) L(\ell)^{*}+\beta(\ell) H(\ell) H(\ell)^{*}\right)^{-1} \\
\mathcal{U}(V, \alpha)(\ell) & :=\mathcal{U}(\mathcal{M}(V, \alpha), V, \alpha)(\ell)
\end{aligned}
$$


and for $V \in \mathbb{H}_{\text {sup }}^{n}$ define the operators $\widetilde{\mathcal{L}}(K, V), \widetilde{\mathcal{I}}(K, V, \beta), \widetilde{\mathcal{U}}(K, V, \beta)$, and $\widetilde{\mathcal{U}}(V, \beta)$ such that

$$
\begin{aligned}
\widetilde{\mathcal{L}}(K, V)(\ell) & :=\int_{\mathcal{S}} g(s, \ell)(A(s)+K(s) L(s)) V(s)(A(s)+K(s) L(s))^{*} \widehat{\mu}_{\mathcal{S}}(d s), \\
\widetilde{\mathcal{I}}(K, V, \beta)(\ell) & :=\int_{\mathcal{S}} g(s, \ell)\left(\beta(s)\left((K(s) H(s))(K(s) H(s))^{*}+G(s) G(s)^{*}\right)+V(s)\right) \widehat{\mu}_{\mathcal{S}}(d s), \\
\widetilde{\mathcal{U}}(K, V, \beta)(\ell) & :=\widetilde{\mathcal{L}}(K, V)(\ell)+\widetilde{\mathcal{I}}(K, 0, \beta)(\ell) . \\
\widetilde{\mathcal{U}}(V, \beta)(\ell) & :=\widetilde{\mathcal{U}}(\mathcal{M}(V, \beta), V, \beta)(\ell) .
\end{aligned}
$$

Remark 6.1.13. Setting $\Gamma(t)=A(t)+K(t) L(t), t \in \mathcal{S}$, and $K \in \mathbb{H}_{\text {sup }}^{p, n}$ in (4.7) one can get that $\mathcal{L}(K, V)=\mathcal{L}(V)$, for all $V \in \mathbb{H}_{1}^{n}$ (it is a slight abuse of notation). Notice also that $\mathcal{L}(K, \cdot), \mathcal{I}(K, \cdot, \alpha), \widetilde{\mathcal{L}}(K, \cdot), \tilde{\mathcal{I}}(K, \cdot, \alpha)$ are positive operators.

The next result follows from the operators defined above.

Proposition 6.1.14. Consider $K \in \mathbb{H}_{\text {sup }}^{p, n}, \alpha \in \mathbb{H}_{1}^{1+} \cap \mathbb{H}_{\text {sup }}^{1+}$ and $\beta \in \mathbb{H}_{\text {sup }}^{1+}$ such that $H(\ell) H(\ell)^{*} \beta(\ell) \geq \epsilon_{H}(\ell) I$ for all $\ell \in \mathcal{S}$, where $\epsilon_{H}$ is as in Assumption 6.1.8. Then $\mathcal{L}(K, \cdot)$, $\mathcal{I}(K, \cdot, \alpha)$ and $\mathcal{U}(K, \cdot, \alpha)$ belong to $\mathbb{B}\left(\mathbb{H}_{1}^{n}, \mathbb{H}_{1}^{n} \cap \mathbb{H}_{\text {sup }}^{n}\right), \mathcal{M}(\cdot, \beta) \in \mathbb{B}\left(\mathbb{H}_{\text {sup }}^{n}, \mathbb{H}_{\text {sup }}^{p, n}\right)$, and $\widetilde{\mathcal{L}}(K, \cdot), \widetilde{\mathcal{I}}(K, \cdot, \beta)$ and $\widetilde{\mathcal{U}}(K, \cdot, \beta)$ belong to $\mathbb{B}\left(\mathbb{H}_{\text {sup }}^{n}, \mathbb{H}_{1}^{n} \cap \mathbb{H}_{\text {sup }}^{n}\right)$.

Proof. It will be shown that $\mathcal{I}(K, V, \alpha) \in \mathbb{H}_{1}^{n} \cap \mathbb{H}_{\text {sup }}^{n}$ whenever $V \in \mathbb{H}_{1}^{n}$. From Fubini's theorem and recalling that $\|K(\ell)\| \leq\|K\|_{\text {sup }} \mu$-almost everywhere in $\mathcal{S}$, and similarly for $\|G(\ell)\|$ and $\|H(\ell)\|$, it follows that

$$
\begin{aligned}
& \left\|\int_{\mathcal{S}} g(s, \ell)\left[\alpha(s)\left((K(s) H(s))(K(s) H(s))^{*}+G(s) G(s)^{*}\right)\right] \mu(d s)\right\|_{1} \\
& \quad \leq\left(\|K\|_{\text {sup }}^{2}\|H\|_{\text {sup }}^{2}+\|G\|_{\text {sup }}^{2}\right) \int_{\mathcal{S}}\left(\int_{\mathcal{S}} g(s, \ell) \mu(d \ell)\right)\|\alpha(s)\| \mu(d s) \\
& \quad=\left(\|K\|_{\text {sup }}^{2}\|H\|_{\text {sup }}^{2}+\|G\|_{\text {sup }}^{2}\right) \int_{\mathcal{S}}\|\alpha(s)\| \mu(d s) \\
& \quad=\left(\|K\|_{\text {sup }}^{2}\|H\|_{\text {sup }}^{2}+\|G\|_{\text {sup }}^{2}\right)\|\alpha\|_{1},
\end{aligned}
$$

since $\int_{\mathcal{S}} g(s, \ell) \mu(d \ell)=1$. For $V \in \mathbb{H}_{1}^{n}$, similarly as in (6.17), it follows that

$$
\begin{aligned}
\left\|\int_{\mathcal{S}} g(s, \ell) V(s) \mu(d s)\right\|_{1} & =\int_{\mathcal{S}}\left(\int_{\mathcal{S}} g(s, \ell) \mu(d \ell)\right)\|V(s)\| \mu(d s) \\
& =\|V\|_{1}
\end{aligned}
$$


since again $\int_{\mathcal{S}} g(s, \ell) \mu(d \ell)=1$. From (6.9), (6.17) and (6.18) one can conclude that

$$
\|\mathcal{I}(K, V, \alpha)\|_{1} \leq\left(\|K\|_{\text {sup }}^{2}\|H\|_{\text {sup }}^{2}+\|G\|_{\text {sup }}^{2}\right)\|\alpha\|_{1}+\|V\|_{1}
$$

From Assumption 6.1.7 one can get that

$$
\|\mathcal{I}(K, V, \alpha)(\ell)\| \leq c\left(\left(\|K\|_{\text {sup }}^{2}\|H\|_{\text {sup }}^{2}+\|G\|_{\text {sup }}^{2}\right)\|\alpha\|_{1}+\|V\|_{1}\right)
$$

showing that indeed $\mathcal{I}(K, \cdot, \alpha) \in \mathbb{B}\left(\mathbb{H}_{1}^{n}, \mathbb{H}_{1}^{n} \cap \mathbb{H}_{\text {sup }}^{n}\right)$. Using similar arguments one gets that $\mathcal{L}(K, \cdot)$ and $\mathcal{U}(K, \cdot, \alpha)$ belong to $\mathbb{B}\left(\mathbb{H}_{1}^{n}, \mathbb{H}_{1}^{n} \cap \mathbb{H}_{\text {sup }}^{n}\right)$. To show that $\mathcal{M}(\cdot, \beta) \in \mathbb{B}\left(\mathbb{H}_{\text {sup }}^{n}, \mathbb{H}_{\text {sup }}^{p, n}\right)$ notice, from $H(\ell) H(\ell)^{*} \beta(\ell) \geq \epsilon_{H}(\ell) I$ and $\frac{\|A(\ell)\|\|L(\ell)\|}{\epsilon_{H}(\ell)} \leq d$, that for $V \in \mathbb{H}_{\text {sup }}^{n}$ and $\ell \in \mathcal{S}$

$$
\|\mathcal{M}(V, \beta)(\ell)\| \leq \frac{1}{\epsilon_{H}(\ell)}\|A(\ell)\|\|L(\ell)\|\|V(\ell)\| \leq d\|V(\ell)\|
$$

and thus it follows that $\|\mathcal{M}(V, \beta)\|_{\text {sup }} \leq d\|V\|_{\text {sup }}$, showing that $\mathcal{M}(\cdot, \beta) \in \mathbb{B}\left(\mathbb{H}_{\text {sup }}^{n}, \mathbb{H}_{\text {sup }}^{p, n}\right)$. Now it will be shown that $\widetilde{\mathcal{I}}(K, \cdot, \beta) \in \mathbb{B}\left(\mathbb{H}_{\text {sup }}^{n}, \mathbb{H}_{1}^{n} \cap \mathbb{H}_{\text {sup }}^{n}\right)$. From Fubini's theorem and recalling again that $\|K(\ell)\| \leq\|K\|_{\text {sup }} \mu$-almost everywhere in $\mathcal{S}$, and similarly for $\|G(\ell)\|$, $\|H(\ell)\|$ and $\|\beta(\ell)\|$, it follows that

$$
\begin{aligned}
& \left\|\int_{\mathcal{S}} g(s, \ell)\left[\beta(s)\left((K(s) H(s))(K(s) H(s))^{*}+G(s) G(s)^{*}\right)\right] \widehat{\mu}_{\mathcal{S}}(d s)\right\|_{1} \\
& \leq\left(\|K\|_{\text {sup }}^{2}\|H\|_{\text {sup }}^{2}+\|G\|_{\text {sup }}^{2}\right)\|\beta\|_{\text {sup }} \int_{\mathcal{S}}\left(\int_{\mathcal{S}} g(s, \ell) \mu(d \ell)\right) \widehat{\mu}_{\mathcal{S}}(d s) \\
& =\left(\|K\|_{\text {sup }}^{2}\|H\|_{\text {sup }}^{2}+\|G\|_{\text {sup }}^{2}\right)\|\beta\|_{\text {sup }} \int_{\mathcal{S}} \widehat{\mu}_{\mathcal{S}}(d s) \\
& =\left(\|K\|_{\text {sup }}^{2}\|H\|_{\text {sup }}^{2}+\|G\|_{\text {sup }}^{2}\right)\|\beta\|_{\text {sup }},
\end{aligned}
$$

since $\int_{\mathcal{S}} g(s, \ell) \mu(d \ell)=1$ and also $\int_{\mathcal{S}} \widehat{\mu}_{\mathcal{S}}(d s)=1$. For $V \in \mathbb{H}_{\text {sup }}^{n}$, similarly as in (6.19), it follows that

$$
\begin{aligned}
\left\|\int_{\mathcal{S}} g(s, \ell) V(s) \widehat{\mu}_{\mathcal{S}}(d s)\right\|_{1} & \leq\|V\|_{\text {sup }} \int_{\mathcal{S}}\left(\int_{\mathcal{S}} g(s, \ell) \mu(d \ell)\right) \widehat{\mu}_{\mathcal{S}}(d s) \\
& =\|V\|_{\text {sup }}
\end{aligned}
$$

since again $\int_{\mathcal{S}} g(s, \ell) \mu(d \ell)=1$ and $\int_{\mathcal{S}} \widehat{\mu}_{\mathcal{S}}(d s)=1$. Then from $(6.14),(6.19)$ and $(6.20)$ it follows that

$$
\|\widetilde{\mathcal{I}}(K, V, \beta)\|_{1} \leq\left(\|K\|_{\text {sup }}^{2}\|H\|_{\text {sup }}^{2}+\|G\|_{\text {sup }}^{2}\right)\|\beta\|_{\text {sup }}+\|V\|_{\text {sup }}
$$


From Assumption 6.1.7 one can easily get that

$$
\|\widetilde{\mathcal{I}}(K, V, \beta)(\ell)\| \leq c\left(\left(\|K\|_{\text {sup }}^{2}\|H\|_{\text {sup }}^{2}+\|G\|_{\text {sup }}^{2}\right)\|\beta\|_{\text {sup }}+\|V\|_{\text {sup }}\right)
$$

showing that indeed $\widetilde{\mathcal{I}}(K, \cdot, \beta) \in \mathbb{B}\left(\mathbb{H}_{\text {sup }}^{n}, \mathbb{H}_{1}^{n} \cap \mathbb{H}_{\text {sup }}^{n}\right)$. Using similar arguments it follows that $\widetilde{\mathcal{L}}(K, \cdot)$ and $\widetilde{\mathcal{U}}(K, \cdot, \beta)$ belong to $\mathbb{B}\left(\mathbb{H}_{\text {sup }}^{n}, \mathbb{H}_{1}^{n} \cap \mathbb{H}_{\text {sup }}^{n}\right)$, completing the proof.

For $\ell \in \mathcal{S}$, define the filtering $\mathcal{S}$-coupled Riccati difference equations as follows,

$$
\begin{aligned}
Y(k+1) & =\mathcal{U}(Y(k), \pi(k)), \quad \text { for } k \in \mathbb{T}_{1}, \\
Y(1) & =\widetilde{\mathcal{U}}(Y(0), \pi(0)),
\end{aligned}
$$

where

$$
Y(0)=V \in \mathbb{H}_{\text {sup }}^{n+},
$$

and define also the filter gains $M(k) \in \mathbb{H}_{\text {sup }}^{p, n}$ as

$$
M(k, \ell)=\mathcal{M}(Y(k), \pi(k))
$$

From (6.21)-(6.23) and from the Definitions (6.8)-(6.16), and recalling Assumption 6.1.6, the filtering algebraic Riccati difference equations (6.21) are given, for $\ell \in \mathcal{S}$, $k \in \mathbb{T}_{1}$, by

$$
\begin{aligned}
Y(k+1, \ell)= & \int_{\mathcal{S}}\left((A(s)+M(k, s) L(s)) Y(k, s)(A(s)+M(k, s) L(s))^{*}\right. \\
& \left.+(G(s)+M(k, s) H(s))(G(s)+M(k, s) H(s))^{*} \pi(k, s)\right) g(s, \ell) \mu(d s), \\
Y(1, \ell)= & \int_{\mathcal{S}}\left((A(s)+M(0, s) L(s)) Y(0, s)(A(s)+M(0, s) L(s))^{*}\right. \\
& \left.+(G(s)+M(0, s) H(s))(G(s)+M(0, s) H(s))^{*}\right) g(s, \ell) \widehat{\mu}_{\mathcal{S}}(d s),
\end{aligned}
$$

Recalling that the operators $\mathcal{L}, \mathcal{I}, \widetilde{\mathcal{L}}, \widetilde{\mathcal{I}}$ defined in (6.8)-(6.9), (6.13)-(6.14) are positive operators, from Proposition 6.1.14 and $Y(0) \in \mathbb{H}_{\text {sup }}^{n+}$ it follows that $Y(k) \in \mathbb{H}_{1}^{n+} \cap \mathbb{H}_{\text {sup }}^{n+}$, for $k \in \mathbb{T}_{1}$.

Define the sequence $U(k)$, for $k \in \mathbb{T}$, as

$$
U(k, \ell):=\left(H(\ell) H(\ell)^{*} \pi(k, \ell)+L(\ell) Y(k, \ell) L(\ell)^{*}\right)^{-1} .
$$


Remark 6.1.15. Notice that after some algebraic manipulations it follows that

$$
\begin{aligned}
A(s) Y(k, s) A(s)^{*}+G(s) G(s)^{*} \pi(k, s)-A(s) Y(k, s) L(s)^{*} U(k, s) L(s) Y(k, s) A(s)^{*} \\
=(A(s)+M(k, s) L(s)) Y(k, s)(A(s)+M(k, s) L(s))^{*}+ \\
\quad(G(s)+M(k, s) H(s))(G(s)+M(k, s) H(s))^{*} \pi(k, s) .
\end{aligned}
$$

From (6.25) and (6.26), one gets that the filtering $\mathcal{S}$-coupled Riccati difference equations (6.21) for $Y(k)$ can also be written, for $\ell \in \mathcal{S}$, as

$$
\begin{aligned}
& Y(k+1, \ell):=\int_{\mathcal{S}}\left(A(s) Y(k, s) A(s)^{*}+G(s) G(s)^{*} \pi(k, s)\right. \\
& \left.\quad-A(s) Y(k, s) L(s)^{*} U(k, s) L(s) Y(k, s) A(s)^{*}\right) g(s, \ell) \mu(d s), \quad \text { for } k \in \mathbb{T}_{1}, \\
& Y(1, \ell):=\int_{\mathcal{S}}\left(A(s) Y(0, s) A(s)^{*}+G(s) G(s)^{*}\right. \\
& \left.\quad-A(s) Y(0, s) L(s)^{*} U(0, s) L(s) Y(0, s) A(s)^{*}\right) g(s, \ell) \widehat{\mu}_{\mathcal{S}}(d s),
\end{aligned}
$$

and the filter gains as

$$
M(k, \ell):=-A(\ell) Y(k, \ell) L(\ell)^{*} U(k, \ell), \quad \text { for } k \in \mathbb{T} .
$$

Remark 6.1.16. From (6.27), $Y(k) \geq 0, k \in \mathbb{T}$, and from Assumption 6.1 .8 it follows that $H(\ell) H(\ell)^{*} \pi(k, \ell)+L(\ell) Y(k, \ell) L(\ell)^{*} \geq H(\ell) H(\ell)^{*} \pi(k, \ell) \geq \epsilon_{H}(\ell) I$, for $\ell \in \mathcal{S}$, so the inverse in (6.25) is indeed well defined.

From (6.25) and (6.28) it results that

$$
M(k, \ell)\left(H(\ell) H(\ell)^{*} \pi(k, \ell)+L(\ell) Y(k, \ell) L(\ell)^{*}\right)=-A(\ell) Y(k, \ell) L(\ell)^{*}
$$

and from Assumption 6.1.8 and Remark 6.1.16, for all $\ell \in \mathcal{S}, k \in \mathbb{T}$,

$$
\begin{aligned}
\|U(k, \ell)\| & =\left\|\left(H(\ell) H(\ell)^{*} \pi(k, \ell)+L(\ell) Y(k, \ell) L(\ell)^{*}\right)^{-1}\right\| \\
& \leq \frac{1}{\epsilon_{H}(\ell)} .
\end{aligned}
$$

The estimator defined next, based on the filtering $\mathcal{S}$-coupled Riccati difference equa- 
tions, is useful when dealing with Problem 6.1.12. Set

$$
\begin{aligned}
\widehat{x}_{e}(k+1) & :=A(\theta(k)) \widehat{x}_{e}(k)+B(\theta(k)) u(k)-M(k, \theta(k))\left(y(k)-L(\theta(k)) \widehat{x}_{e}(k)\right) \\
\widehat{x}_{e}(0) & :=E\left(x_{0}\right)=\zeta,
\end{aligned}
$$

where $M(k, \ell)$ is defined from (6.23).

Remark 6.1.17. As in Remark 6.1.11 one gets that $\widehat{x}_{e}(k)$ is $\mathcal{F}_{k-1}$-measurable for $k \in \mathbb{N}_{1}$.

The error associated with the estimator given in (6.31) is defined by

$$
\widetilde{x}_{e}(k):=x(k)-\widehat{x}_{e}(k)
$$

and from (6.1) and (6.31) one gets that

$$
\widetilde{x}_{e}(k+1)=(A(\theta(k))+M(k, \theta(k)) L(\theta(k))) \widetilde{x}_{e}(k)+(G(\theta(k))+M(k, \theta(k)) H(\theta(k))) w(k) .
$$

In what follows define $Y(0)=V:=\left(\mathbb{Q}_{0}-\zeta \zeta^{\prime}\right)=E\left(\left(x_{0}-\zeta\right)\left(x_{0}-\zeta\right)^{\prime}\right) \geq 0$. Recall that from Remark 3.1.5, $Y(0)$ means $Y(0, \ell), \ell \in \mathcal{S}$. Clearly $Y(0) \in \mathbb{H}_{\text {sup }}^{n+}$ since $\|Y(0)\| \leq$ $E\left(\left\|x_{0}-\zeta\right\|^{2}\right)=\operatorname{tr}\left(\mathbb{Q}_{0}-\zeta \zeta^{\prime}\right)<\infty$.

The next result gives an interpretation for $Y(k)$ in terms of the second moment for $\widetilde{x}_{e}(k)$. Notice that from the definition of $Y(0)$ stated above and (6.32), it follows that $Y(0)=E\left(\widetilde{x}_{e}(0) \widetilde{x}_{e}(0)^{*}\right)$.

Proposition 6.1.18. The filtering $\mathcal{S}$-coupled Riccati difference equations defined in (6.21) satisfy, for $k \in \mathbb{T}$,

$$
Y(k+1, \ell)=E\left(\widetilde{x}_{e}(k+1) \widetilde{x}_{e}(k+1)^{*} g(\theta(k), \ell)\right) .
$$

Proof. The result will be shown by induction on $k$. From Fubini's theorem, (6.24), (6.33) and recalling that $E(w(k))=0, E\left(w(k) w(k)^{*}\right)=I, k \in \mathbb{T}$, and that $w(0), \theta_{0}, x_{0}$ are independent random variables, it follows for $k=0$ that

$$
\begin{aligned}
Y(1, \ell)= & \int_{\mathcal{S}}\left((A(s)+M(0, s) L(s)) Y(0, s)(A(s)+M(0, s) L(s))^{*}+(G(s)+M(0, s) H(s))\right. \\
& \left.(G(s)+M(0, s) H(s))^{*}\right) g(s, \ell) \widehat{\mu}_{\mathcal{S}}(d s) \\
= & \int_{\mathcal{S}}\left((A(s)+M(0, s) L(s)) E\left(\widetilde{x}_{e}(0) \widetilde{x}_{e}(0)^{*}\right)(A(s)+M(0, s) L(s))^{*}+\right.
\end{aligned}
$$




$$
\begin{aligned}
& +(A(s)+M(0, s) L(s)) E\left(\widetilde{x}_{e}(0)\right) E\left(w(0)^{*}\right)(G(s)+M(0, s) H(s))^{*}+ \\
& +(G(s)+M(0, s) H(s)) E(w(0)) E\left(\widetilde{x}_{e}(0)^{*}\right)(A(s)+M(0, s) L(s))^{*}+ \\
& \left.+(G(s)+M(0, s) H(s)) E\left(w(0) w(0)^{*}\right)(G(s)+M(0, s) H(s))^{*}\right) g(s, \ell) \widehat{\mu}_{\mathcal{S}}(d s) \\
= & E\left(\int _ { \mathcal { S } } \left((A(s)+M(0, s) L(s))\left(\widetilde{x}_{e}(0) \widetilde{x}_{e}(0)^{*}\right)(A(s)+M(0, s) L(s))^{*}+\right.\right. \\
& +(A(s)+M(0, s) L(s))\left(\widetilde{x}_{e}(0) w(0)^{*}\right)(G(s)+M(0, s) H(s))^{*}+ \\
& +(G(s)+M(0, s) H(s))\left(w(0) \widetilde{x}_{e}(0)^{*}\right)(A(s)+M(0, s) L(s))^{*}+ \\
& \left.\left.+(G(s)+M(0, s) H(s))\left(w(0) w(0)^{*}\right)(G(s)+M(0, s) H(s))^{*}\right) g(s, \ell) \widehat{\mu}_{\mathcal{S}}(d s)\right) \\
= & E\left(\int_{\mathcal{S}} \widetilde{x}_{e}(1) \widetilde{x}_{e}(1)^{*} g(s, \ell) \widehat{\mu}_{\mathcal{S}}(d s)\right) \\
= & E\left(\widetilde{x}_{e}(1) \widetilde{x}_{e}(1)^{*} g\left(\theta_{0}, \ell\right)\right) .
\end{aligned}
$$

Suppose the result holds for $k>0$. Recall that $x(k), \widehat{x}_{e}(k)$ are $\mathcal{F}_{k-1}$-measurable (see Remarks 6.1.11 and 6.1.17), $\widetilde{x}_{e}(k)=x(k)-\widehat{x}_{e}(k)$, and that by Assumption 6.1.4 the noise $\{w(k) ; k \in \mathbb{T}\}$, the Markov chain $\{\theta(k) ; k \in \mathbb{T}\}$ and the initial condition $x_{0}$ are mutually independent, and thus, in particular, $w(k)$ is independent of $x(k)$ and $\widehat{x}_{e}(k)$. From (6.24), (6.33) and Fubini's theorem, it results that

$$
\begin{aligned}
Y( & +1, \ell)=\int_{\mathcal{S}}\left[(A(s)+M(k, s) L(s)) E\left(\widetilde{x}_{e}(k) \widetilde{x}_{e}(k)^{*} g(\theta(k-1), s)\right)(A(s)+M(k, s) L(s))^{*}\right. \\
& \left.+(G(s)+M(k, s) H(s))(G(s)+M(k, s) H(s))^{*} \pi(k, s)\right] g(s, \ell) \mu(d s) \\
= & \int_{\mathcal{S}}\left((A(s)+M(k, s) L(s)) E\left(\widetilde{x}_{e}(k) \widetilde{x}_{e}(k)^{*} g(\theta(k-1), s)\right)(A(s)+M(k, s) L(s))^{*}\right. \\
& +(A(s)+M(k, s) L(s)) E\left(\widetilde{x}_{e}(k) g(\theta(k-1), s)\right) E\left(w(k)^{*}\right)(G(s)+M(k, s) H(s))^{*} \\
& +(G(s)+M(k, s) H(s)) E(w(k)) E\left(\widetilde{x}_{e}(k)^{*} g(\theta(k-1), s)\right)(A(s)+M(k, s) L(s))^{*} \\
& \left.+(G(s)+M(k, s) H(s)) E\left(w(k) w(k)^{*}\right) E(g(\theta(k-1), s))(G(s)+M(k, s) H(s))^{*}\right) \\
& g(s, \ell) \mu(d s) \\
= & E\left(\int_{\mathcal{S}}\left((A(s)+M(k, s) L(s)) \widetilde{x}_{e}(k)+(G(s)+M(k, s) H(s)) w(k)\right)\right. \\
& \left.\left((A(s)+M(k, s) L(s)) \widetilde{x}_{e}(k)+(G(s)+M(k, s) H(s)) w(k)\right)^{*} g(s, \ell) g(\theta(k-1), s) \mu(d s)\right) \\
= & E\left(E \left(\left((A(\theta(k))+M(k, \theta(k)) L(\theta(k))) \widetilde{x}_{e}(k)+(G(\theta(k))+M(k, \theta(k)) H(\theta(k))) w(k)\right)\right.\right. \\
& \left((A(\theta(k))+M(k, \theta(k)) L(\theta(k))) \widetilde{x}_{e}(k)+(G(\theta(k))+M(k, \theta(k)) H(\theta(k))) w(k)\right)^{*}
\end{aligned}
$$




$$
\begin{aligned}
& \left.\left.g(\theta(k), \ell) \mid \mathcal{F}_{k-1}\right)\right) \\
= & E\left(\widetilde{x}_{e}(k+1) \widetilde{x}_{e}(k+1)^{*} g(\theta(k), \ell)\right),
\end{aligned}
$$

and the result follows.

The next result provides an orthogonality property between $\widetilde{x}_{e}(k+1)$ and $\widehat{x}(k+1)$ for any estimator given by (6.5).

Proposition 6.1.19. For $\widehat{x}(k), \widehat{x}_{e}(k), \widetilde{x}_{e}(k)$ given by (6.5), (6.31) and (6.32), respectively, and $k \in \mathbb{T}, \ell \in \mathcal{S}$, it holds that

$$
\begin{aligned}
E\left(\widetilde{x}_{e}(k+1) \widehat{x}(k+1)^{*} g(\theta(k), \ell)\right) & =0, \\
E\left(\widetilde{x}_{e}(k+1) \widehat{x}_{e}(k+1)^{*} g(\theta(k), \ell)\right) & =0 .
\end{aligned}
$$

Proof. The result stated in (6.35) will be proved by induction on $k$. For notational simplicity in what follows it is omitted in $\widehat{A}(k, \ell), \widehat{B}(k, \ell), M(k, \ell)$ the dependence on $k$. Recall that $w(0), x_{0}$ and $\theta_{0}$ are independent random variables, $\widehat{x}(0)$ is deterministic, $E\left(\widetilde{x}_{e}(0)\right)=0, E(w(0))=0, E\left(w(0) w(0)^{\prime}\right)=I$, and also that, by Assumption 6.1.6, $G\left(\theta_{0}\right) H\left(\theta_{0}\right)^{*}=0$. From $(6.1)$ and $(6.32)$ it follows that $y(0)=L\left(\theta_{0}\right)\left(\widetilde{x}_{e}(0)+\widehat{x}_{e}(0)\right)+$ $H\left(\theta_{0}\right) w(0)$. Thus, from Fubini's theorem, Assumption 6.1.8, (6.5), (6.29) and (6.33) it follows for $k=0$ that

$$
\begin{aligned}
E\left(\widetilde{x}_{e}(1) \widehat{x}(1)^{*} g\left(\theta_{0}, \ell\right)\right) & \\
= & E\left(\left(\left(A\left(\theta_{0}\right)+M\left(\theta_{0}\right) L\left(\theta_{0}\right)\right) \widetilde{x}_{e}(0)+\left(G\left(\theta_{0}\right)+M\left(\theta_{0}\right) H\left(\theta_{0}\right)\right) w(0)\right)\right. \\
& \left.\left(\widehat{A}\left(\theta_{0}\right) \widehat{x}(0)+\widehat{B}\left(\theta_{0}\right)\left(L\left(\theta_{0}\right)\left(\widetilde{x}_{e}(0)+\widehat{x}_{e}(0)\right)+H\left(\theta_{0}\right) w(0)\right)\right)^{*} g\left(\theta_{0}, \ell\right)\right) \\
= & \int_{\mathcal{S}}\left(( A ( s ) + M ( s ) L ( s ) ) \left(E\left(\widetilde{x}_{e}(0)\right) \widehat{x}(0)^{*} \widehat{A}(s)^{*}+E\left(\widetilde{x}_{e}(0) \widetilde{x}_{e}(0)^{*}\right) L(s)^{*} \widehat{B}(s)^{*}\right.\right. \\
& \left.+E\left(\widetilde{x}_{e}(0)\right) \widehat{x}_{e}(0)^{*} L(s)^{*} \widehat{B}(s)^{*}+E\left(\widetilde{x}_{e}(0)\right) E\left(w(0)^{*}\right) H(s)^{*} \widehat{B}(s)^{*}\right) \\
& +(G(s)+M(s) H(s))\left(E(w(0)) \widehat{x}(0)^{*} \widehat{A}(s)^{*}+E(w(0)) E\left(\widetilde{x}_{e}(0)^{*}\right) L(s)^{*} \widehat{B}(s)^{*}\right. \\
& \left.\left.+E(w(0)) \widehat{x}_{e}(0)^{*} L(s)^{*} \widehat{B}(s)^{*}+E\left(w(0) w(0)^{*}\right) H(s)^{*} \widehat{B}(s)^{*}\right)\right) g(s, \ell) \widehat{\mu}_{\mathcal{S}}(d s) \\
= & \int_{\mathcal{S}}\left((A(s)+M(s) L(s)) Y(0) L(s)^{*}+M(s) H(s) H(s)^{*}\right) \widehat{B}(s)^{*} g(s, \ell) \widehat{\mu}_{\mathcal{S}}(d s) \\
= & \int_{\mathcal{S}}\left(A(s) Y(0) L(s)^{*}+M(s)\left(L(s) Y(0) L(s)^{*}+H(s) H(s)^{*}\right)\right) \widehat{B}(s)^{*} g(s, \ell) \widehat{\mu}_{\mathcal{S}}(d s) \\
= & 0 .
\end{aligned}
$$


Suppose the result holds for $k>0$. Notice that $x(k), \widehat{x}(k)$ and $\widehat{x}_{e}(k)$ are $\mathcal{F}_{k-1^{-}}$ measurable (see Remarks 6.1.11 and 6.1.17) and that by Assumption 6.1.4 the noise $\{w(k) ; k \in \mathbb{T}\}$, the Markov chain $\{\theta(k) ; k \in \mathbb{T}\}$ and the initial condition $x_{0}$ are mutually independent. The proof will be based on several steps.

Step 1. From the induction hypothesis on $k$, it follows from Fubini's theorem that

$$
\begin{aligned}
E( & \left.\left((A(\theta(k))+M(\theta(k)) L(\theta(k))) \widetilde{x}_{e}(k)\right)(\widehat{A}(\theta(k)) \widehat{x}(k))^{*} g(\theta(k), \ell)\right) \\
& =E\left(E\left(\left((A(\theta(k))+M(\theta(k)) L(\theta(k))) \widetilde{x}_{e}(k)\right)(\widehat{A}(\theta(k)) \widehat{x}(k))^{*} g(\theta(k), \ell) \mid \mathcal{F}_{k-1}\right)\right) \\
& =E\left(\int_{\mathcal{S}}(A(s)+M(s) L(s)) \widetilde{x}_{e}(k) \widehat{x}(k)^{*} \widehat{A}(s)^{*} g(s, \ell) g(\theta(k-1), s) \mu(d s)\right) \\
& =\int_{\mathcal{S}}(A(s)+M(s) L(s)) E\left(\widetilde{x}_{e}(k) \widehat{x}(k)^{*} g(\theta(k-1), s)\right) \widehat{A}(s)^{*} g(s, \ell) \mu(d s) \\
& =0 .
\end{aligned}
$$

Step 2. Recalling that $E(w(k))=0$ and noticing that, from Assumption 6.1.4, $w(k)$ is independent of $\theta(k)$ and $\widehat{x}(k)$ (since $\widehat{x}(k)$ can be written in function of $x_{0}$, and $\theta(j)$ and $w(j), j=0, \ldots, k-1)$, it follows that

$$
\begin{aligned}
& E\left(((G(\theta(k))+M(\theta(k)) H(\theta(k))) w(k))(\widehat{A}(\theta(k)) \widehat{x}(k))^{*} g(\theta(k), \ell)\right) \\
& \quad=E\left(((G(\theta(k))+M(\theta(k)) H(\theta(k))) E(w(k)))(\widehat{A}(\theta(k)) \widehat{x}(k))^{*} g(\theta(k), \ell)\right) \\
& \quad=0,
\end{aligned}
$$

and similarly, noticing that $\widetilde{x}_{e}(k)=x(k)-\widehat{x}_{e}(k)$ and $w(k)$ is independent of $\theta(k), \widehat{x}_{e}(k)$ and $x(k)$,

$$
\begin{aligned}
& E\left(\left((A(\theta(k))+M(\theta(k)) L(\theta(k))) \widetilde{x}_{e}(k)\right)(\widehat{B}(\theta(k))(H(\theta(k)) w(k)))^{*} g(\theta(k), \ell)\right) \\
& \quad=E\left(\left((A(\theta(k))+M(\theta(k)) L(\theta(k))) \widetilde{x}_{e}(k)\right)(\widehat{B}(\theta(k))(H(\theta(k)) E(w(k))))^{*} g(\theta(k), \ell)\right) \\
& \quad=0 .
\end{aligned}
$$

Step 3. From (6.1) and (6.32) it holds that $y(k)=L(\theta(k))\left(\widetilde{x}_{e}(k)+\widehat{x}_{e}(k)\right)+H(\theta(k)) w(k)$. Then, from the induction hypothesis on $k$, Step 2 , and recalling that $\widehat{x}(k)$ and $\widehat{x}_{e}(k)$ are $\mathcal{F}_{k-1}$-measurable, one gets, by repeating similar arguments as in Step 1, that

$$
E\left(\left((A(\theta(k))+M(\theta(k)) L(\theta(k))) \widetilde{x}_{e}(k)\right)(\widehat{B}(\theta(k)) y(k))^{*} g(\theta(k), \ell)\right)
$$




$$
\begin{aligned}
= & \int_{\mathcal{S}}(A(s)+M(s) L(s)) E\left(\widetilde{x}_{e}(k) \widetilde{x}_{e}(k)^{*} g(\theta(k-1), s)\right) L(s)^{*} \widehat{B}(s)^{*} g(s, \ell) \mu(d s) \\
& +\int_{\mathcal{S}}(A(s)+M(s) L(s)) E\left(\widetilde{x}_{e}(k) \widehat{x}_{e}(k)^{*} g(\theta(k-1), s)\right) L(s)^{*} \widehat{B}(s)^{*} g(s, \ell) \mu(d s) \\
= & \int_{\mathcal{S}}(A(s)+M(s) L(s)) Y(k, s) L(s)^{*} \widehat{B}(s)^{*} g(s, \ell) \mu(d s) .
\end{aligned}
$$

Step 4. Using similar arguments as in Step $2(w(k)$ is independent of $\theta(k)$, and $x(k)$ since it can be written in function of $x_{0}$, and $\theta(j)$ and $\left.w(j), j=0, \ldots, k-1\right)$ it follows that

$$
\begin{aligned}
& E\left(((G(\theta(k))+M(\theta(k)) H(\theta(k))) w(k))(\widehat{B}(\theta(k)) L(\theta(k)) x(k))^{*} g(\theta(k), \ell)\right) \\
& \quad=E\left(((G(\theta(k))+M(\theta(k)) H(\theta(k))) E(w(k)))(\widehat{B}(\theta(k)) L(\theta(k)) x(k))^{*} g(\theta(k), \ell)\right) \\
& \quad=0,
\end{aligned}
$$

and recalling that $E\left(w(k) w(k)^{\prime}\right)=I, y(k)=L(\theta(k)) x(k)+H(\theta(k)) w(k)$, and, by Assumption 6.1.6, $G(\theta(k)) H(\theta(k))^{*}=0$, it follows that

$$
\begin{aligned}
E( & \left.((G(\theta(k))+M(\theta(k)) H(\theta(k))) w(k))(\widehat{B}(\theta(k)) y(k))^{*} g(\theta(k), \ell)\right) \\
& =E\left((G(\theta(k))+M(\theta(k)) H(\theta(k))) E\left(w(k) w(k)^{*}\right) H(\theta(k))^{*} \widehat{B}(\theta(k))^{*} g(\theta(k), \ell)\right) \\
& =\int_{\mathcal{S}} M(s) H(s) H(s)^{*} \widehat{B}(s)^{*} E(g(\theta(k-1), s)) g(s, \ell) \mu(d s) \\
& =\int_{\mathcal{S}} M(s) H(s) H(s)^{*} \pi(k, s) \widehat{B}(s)^{*} g(s, \ell) \mu(d s) .
\end{aligned}
$$

From Steps 1 to 4, (6.29) and Assumption 6.1.8, it follows that

$$
\begin{aligned}
E & \left(\widetilde{x}_{e}(k+1) \widehat{x}(k+1)^{*} g(\theta(k), \ell)\right) \\
& =\int_{\mathcal{S}}\left((A(s)+M(s) L(s)) Y(k, s) L(s)^{*}+M(s) H(s) H(s)^{*} \pi(k, s)\right) \widehat{B}(s)^{*} g(s, \ell) \mu(d s) \\
& =\int_{\mathcal{S}}\left(A(s) Y(k, s) L(s)^{*}+M(s)\left(L(s) Y(k, s) L(s)^{*}+H(s) H(s)^{*} \pi(k, s)\right)\right) \widehat{B}(s)^{*} g(s, \ell) \mu(d s) \\
& =\int_{\mathcal{S}}\left(A(s) Y(k, s) L(s)^{*}-A(s) Y(k, s) L(s)^{*}\right) \widehat{B}(s)^{*} g(s, \ell) \mu(d s) \\
& =0
\end{aligned}
$$

and (6.35) follows. 
To show (6.36) notice from (6.31) and (6.5) that

$$
\widehat{x}_{e}(k+1)=\widehat{A}_{e}(k, \theta(k)) \widehat{x}_{e}(k)+\bar{B}_{e}(k, \theta(k)) \widehat{x}(k)+\widehat{B}_{e}(k, \theta(k)) y(k),
$$

where $\widehat{A}_{e}(k, \ell)=A(\ell)+M(k, \ell) L(\ell), \widehat{B}_{e}(k, \ell)=-M(k, \ell)$ and $\bar{B}_{e}(k, \ell)=B(\ell) \widehat{C}(k, \ell)$. Notice also that (6.38) has a similar structure as in (6.5), with an extra term given by $\bar{B}_{e}(k, \theta(k)) \widehat{x}(k)$. Considering (6.37), (6.38), and $E\left(\widetilde{x}_{e}(k+1) \widehat{x}_{e}(k+1)^{*} g(\theta(k), \ell)\right)$ one gets, following a similar procedure as in the proof of (6.35), that (6.36) will hold if it is shown that $E\left(\widetilde{x}_{e}(k+1)\left(\bar{B}_{e}(k, \theta(k)) \widehat{x}(k)\right)^{*} g(\theta(k), \ell)\right)=0$. From (6.35) it follows that this will hold if $E\left(\widehat{A}_{e}(k, \theta(k)) \widetilde{x}_{e}(k) \widehat{x}(k)^{*} \bar{B}_{e}(k, \theta(k))^{*} g(\theta(k), \ell)\right)=0$ and $E((G(\theta(k))+M(\theta(k))$ $\left.H(\theta(k))) w(k) \widehat{x}(k)^{*} \bar{B}_{e}(k, \theta(k))^{*} g(\theta(k), \ell)\right)=0$. From the independence of $w(k)$ and $\theta(k)$, $\widehat{x}(k)$, and recalling that $E(w(k))=0$ it follows that

$$
\begin{aligned}
& E\left((G(\theta(k))+M(\theta(k)) H(\theta(k))) w(k) \widehat{x}(k)^{*} \bar{B}_{e}(k, \theta(k))^{*} g(\theta(k), \ell)\right) \\
& \quad=E\left((G(\theta(k))+M(\theta(k)) H(\theta(k))) E(w(k)) \widehat{x}(k)^{*} \bar{B}_{e}(k, \theta(k))^{*} g(\theta(k), \ell)\right) \\
& \quad=0 .
\end{aligned}
$$

From (6.35) and similar reasoning as in Step 1 one gets that for $k>0$,

$$
\begin{aligned}
E( & \left.\widehat{A}_{e}(k, \theta(k)) \widetilde{x}_{e}(k) \widehat{x}(k)^{*} \bar{B}_{e}(k, \theta(k))^{*} g(\theta(k), \ell)\right) \\
& =\int_{\mathcal{S}} \widehat{A}_{e}(k, s) E\left(\widetilde{x}_{e}(k) \widehat{x}(k)^{*} g(\theta(k-1), s) \bar{B}_{e}(k, s)\right) g(s, \ell) \mu(d s) \\
& =0 .
\end{aligned}
$$

For $k=0$ one gets, recalling that $E\left(\widetilde{x}_{e}(0)\right)=0$ and that $\widehat{x}(0)$ is deterministic, that

$$
\begin{aligned}
E & \left(\widehat{A}_{e}\left(0, \theta_{0}\right) \widetilde{x}_{e}(0) \widehat{x}(0)^{*} \bar{B}_{e}\left(0, \theta_{0}\right)^{*} g\left(\theta_{0}, \ell\right)\right) \\
& =\int_{\mathcal{S}} \widehat{A}_{e}(0, s) E\left(\widetilde{x}_{e}(0)\right) \widehat{x}(0)^{*} \bar{B}_{e}(0, s)^{*} g(s, \ell) \widehat{\mu}_{\mathcal{S}}(d s) \\
& =0
\end{aligned}
$$

completing the proof of (6.36).

Combining the previous results, one gets the following proposition. 
Proposition 6.1.20. Let $v(k)$ and $Y(k)$ be as in (6.7) and (6.21), respectively. Then

$$
E\left(\|v(0)\|^{2}\right) \geq \operatorname{tr}(Y(0))
$$

and for every $k=1, \ldots, \tau$,

$$
E\left(\|v(k)\|^{2}\right) \geq \int_{\mathcal{S}} \operatorname{tr}(Y(k, s)) \mu(d s) .
$$

Proof. The case $k=0$ follows from Proposition 1.1.1 (c) by Davis and Vinter (1985). Consider $k>0$. From Fubini's theorem and Propositions 6.1.18 and 6.1.19, and recalling that $\widetilde{x}_{e}(k)=x(k)-\widehat{x}_{e}(k)$, it follows that

$$
\begin{aligned}
E( & \left.\|v(k)\|^{2}\right)=E\left(\|x(k)-\widehat{x}(k)\|^{2}\right) \\
& =E\left(\operatorname{tr}\left(\left(\widetilde{x}_{e}(k)+\widehat{x}_{e}(k)-\widehat{x}(k)\right)\left(\widetilde{x}_{e}(k)+\widehat{x}_{e}(k)-\widehat{x}(k)\right)^{*}\right)\right) \\
& =E\left(\int_{\mathcal{S}} \operatorname{tr}\left(\left(\widetilde{x}_{e}(k)+\widehat{x}_{e}(k)-\widehat{x}(k)\right)\left(\widetilde{x}_{e}(k)+\widehat{x}_{e}(k)-\widehat{x}(k)\right)^{*} g(\theta(k-1), s)\right) \mu(d s)\right) \\
& =\int_{\mathcal{S}} \operatorname{tr}\left(E\left(\left(\widetilde{x}_{e}(k)+\widehat{x}_{e}(k)-\widehat{x}(k)\right)\left(\widetilde{x}_{e}(k)+\widehat{x}_{e}(k)-\widehat{x}(k)\right)^{*} g(\theta(k-1), s)\right)\right) \mu(d s) \\
& =\int_{\mathcal{S}} \operatorname{tr}(Y(k, s)) \mu(d s)+E\left(\left\|\widehat{x}_{e}(k)-\widehat{x}(k)\right\|^{2}\right) \\
& +2 \int_{\mathcal{S}} \operatorname{tr}\left(E\left(\widetilde{x}_{e}(k)\left(\widehat{x}_{e}(k)-\widehat{x}(k)\right)^{*} g(\theta(k-1), s)\right)\right) \mu(d s) \\
& =\int_{\mathcal{S}} \operatorname{tr}(Y(k, s)) \mu(d s)+E\left(\left\|\widehat{x}_{e}(k)-\widehat{x}(k)\right\|^{2}\right) \\
& \geq \int_{\mathcal{S}} \operatorname{tr}(Y(k, s)) \mu(d s) .
\end{aligned}
$$

The next result presents a solution to the finite-horizon filtering problem. The optimal filter is obtained from the filtering $\mathcal{S}$-coupled Riccati difference equations (6.27)-(6.28).

Theorem 6.1.21. An optimal solution for the filtering problem stated in Problem 6.1.12 is

$$
\begin{aligned}
& \widehat{A}^{o p}(k, \theta(k))=A(\theta(k))+M(k, \theta(k)) L(\theta(k))+B(\theta(k)) \widehat{C}(\theta(k)) \\
& \widehat{B}^{o p}(k, \theta(k))=-M(k, \theta(k)),
\end{aligned}
$$


with $\widehat{C}(k) \in \mathbb{H}_{\text {sup }}^{n, m}$ arbitrary, and $\widehat{x}_{0}=\zeta$. The optimal cost for the filtering problem is

$$
\sum_{k=1}^{\tau} \int_{\mathcal{S}} \operatorname{tr}(Y(k, s)) \mu(d s)+\operatorname{tr}(Y(0))
$$

Proof. Note that from Proposition 6.1.14 it follows that $M(k) \in \mathbb{H}_{\text {sup }}^{p, n}$ and thus $\widehat{A}^{o p}(k) \in$ $\mathbb{H}_{\text {sup }}^{n}, \widehat{B}^{o p}(k) \in \mathbb{H}_{\text {sup }}^{p, n}$. From Proposition 6.1.20 it can be concluded that the filter defined in (6.31) is an optimal filter for the finite-horizon filtering problem and the optimal cost is given by (6.39).

\subsection{Infinite-Horizon Filtering}

In this section the design of an infinite-horizon minimum mean square linear Markov jump filter for a MJLS is considered. The optimal filter is derived in Theorem 6.2.7 in terms of the stabilizing positive semi-definite solution of the filtering $\mathcal{S}$-coupled algebraic Riccati equations. Sufficient conditions for the existence and uniqueness of this stabilizing positive semi-definite solution for the filtering $\mathcal{S}$-coupled algebraic Riccati equations, based on the concepts of (stochastic) stabilizability and detectability, are presented in Appendix A.2. In Theorem A.2.8 it is shown that, under the assumption of $(A, G)$ being stochastically stabilizable and $(L, A)$ stochastically detectable, there exists a unique stabilizing solution (in the $\mu$-almost everywhere sense) $Y(\ell), \ell \in \mathcal{S}$, for the filtering $\mathcal{S}$-coupled algebraic Riccati equations.

\subsubsection{Problem Statement}

On the filtered probability space $\left(\Omega, \mathcal{F},\left\{\mathcal{F}_{k}\right\}_{k \in \mathbb{N}}, \mathcal{P}\right)$ consider the following discrete-time Markov jump linear system

$$
\left\{\begin{aligned}
x(k+1) & =A(\theta(k)) x(k)+B(\theta(k)) u(k)+G(\theta(k)) w(k) \\
y(k) & =L(\theta(k)) x(k)+H(\theta(k)) w(k)
\end{aligned}\right.
$$

Recall that the assumptions stated in Section 3.3 hold, that is, Assumption 3.2.2 holds, $E\left(x_{0}\right):=\zeta, E\left(x_{0} x_{0}^{*}\right):=\mathbb{Q}_{0}$, and thus $E\left(\left\|x_{0}-\zeta\right\|^{2}\right)=\operatorname{tr}\left(\mathbb{Q}_{0}-\zeta \zeta^{*}\right)<\infty$. Assume also the Assumptions 6.1 .4 to 6.1 .8 , stated in Section 6.1, and the hypothesis below.

Assumption 6.2.1. $\{\theta(k) ; k \in \mathbb{N}\}$ is uniformly ergodic (see Meyn and Tweedie, 2009, $p$. 393). 
In this section define the sequence $\{\pi(k) ; k \in \mathbb{N}\}$ as

$$
\pi(k):=T^{k} \nu
$$

where the operator $T$ is as in (3.5), and

$$
T^{k} \nu:=T\left(T^{k-1} \nu\right), \quad k=1,2, \ldots
$$

and $T^{0}:=I$. From (6.41) it follows, for $k \in \mathbb{N}$, that

$$
\pi(k+1, \ell)=\int_{\mathcal{S}} g(s, \ell) \pi(k, s) \mu(d s)
$$

Assumption 6.2.1 is required to assure the uniform convergence of the probability density function $\pi(k)$ to a limit value. As shown in Theorem 16.0.2 by Meyn and Tweedie (2009), an aperiodic and irreducible Markov chain is uniformly ergodic if and only if it satisfies the Doeblin's condition (see Meyn and Tweedie, 2009, p. 394). This convergence is used for the convergence of the solution of the recursive filtering $\mathcal{S}$-coupled Riccati difference equations to the stabilizing solution of the filtering $\mathcal{S}$-coupled algebraic Riccati equations derived in Appendix A.2, and is required in many situations involving convergence of this kind (for instance, in Markov Decision Processes with undiscounted criteria (see Hernández-Lerma and Lasserre, 1999)). Notice that this assumption is always satisfied for the irreducible aperiodic finite state Markov chain case.

Proposition 6.2.2. The following recursive expression for $\pi(k), k \in \mathbb{N}$, holds,

$$
\pi(k+1, \ell)=E(g(\theta(k), \ell)) .
$$

Proof. The result will be shown by induction on $k$. For $k=0$ one gets from (6.41) and (3.5) that $\pi(1, \ell)=T \nu(\ell)=\int_{\mathcal{S}} g(t, \ell) \nu(t) \mu(d t)=E\left(g\left(\theta_{0}, \ell\right)\right)$. Suppose now that the result holds for $k>0$. Then one gets from Fubini's theorem, (3.5), (3.11) and (6.41) that

$$
\begin{aligned}
\pi(k+1, \ell) & =T(\pi(k))(\ell)=\int_{\mathcal{S}} g(t, \ell) E(g(\theta(k-1), t)) \mu(d t) \\
& =E\left(\int_{\mathcal{S}} g(t, \ell) g(\theta(k-1), t) \mu(d t)\right)=E(E(g(\theta(k), \ell) \mid \theta(k-1))) \\
& =E(g(\theta(k), \ell)),
\end{aligned}
$$

showing (6.43). 
The next proposition states that $\pi(k)$ is the probability density function of $\theta(k)$ with respect to $\mu$, for $k \in \mathbb{N}_{1}$.

Proposition 6.2.3. For $k \in \mathbb{N}, B \in \mathcal{B}(\mathcal{S})$, it holds that

$$
\mathcal{P}(\theta(k+1) \in B)=\int_{B} \pi(k+1, \ell) \mu(d \ell)
$$

Proof. From Fubini's theorem, (3.11) and (6.43), for $k \in \mathbb{N}$,

$$
\begin{aligned}
\mathcal{P}(\theta(k+1) \in B) & =E(\mathcal{P}(\theta(k+1) \in B \mid \theta(k)))=E\left(\int_{B} g(\theta(k), \ell) \mu(d \ell)\right) \\
& =\int_{B} E(g(\theta(k), \ell)) \mu(d \ell)=\int_{B} \pi(k+1, \ell) \mu(d \ell) .
\end{aligned}
$$

Thus, for $k \in \mathbb{N}, \pi(k+1)$ is the density function of $\theta(k+1)$ with respect to $\mu$.

Assumption 3.2.2 assures that the initial distribution of $\theta(0)$ has a density $\nu$ with respect to $\mu$. Then from Proposition 6.1.3, by recurrence, $\theta(k)$ has a density $\pi(k)=T^{k} \nu$ with respect to $\mu$, for all $k \in \mathbb{N}$. The next proposition follows from (6.41) and is similar to Proposition 6.1.9.

Proposition 6.2.4. It holds that $\pi(k) \in \mathbb{H}_{1}^{1+} \cap \mathbb{H}_{\text {sup }}^{1+}$ for $k \in \mathbb{N}_{1}$. Moreover, $\|\pi(k)\|_{1}=1$.

Proof. The result will be shown by induction on $k$. From Fubini's theorem, (6.42), and recalling that $\pi(0)=\nu \in \mathbb{H}_{1}^{1+}$ and $\|\nu\|_{1}=1$, the result holds for $k=1$ since,

$$
\begin{aligned}
\|\pi(1)\|_{1} & =\int_{\mathcal{S}} \int_{\mathcal{S}} \pi(0, s) g(s, \ell) \mu(d s) \mu(d \ell)=\int_{\mathcal{S}} \nu(s) \int_{\mathcal{S}} g(s, \ell) \mu(d \ell) \mu(d s) \\
& =\int_{\mathcal{S}} \nu(s) \mu(d s)=1
\end{aligned}
$$

since $\int_{\mathcal{S}} g(s, \ell) \mu(d \ell)=1$, and from Assumption 6.1.7 and (6.42) it follows that

$$
\begin{aligned}
\|\pi(1, \ell)\| & =\left\|\int_{\mathcal{S}} \pi(0, s) g(s, \ell) \mu(d s)\right\|=\left\|\int_{\mathcal{S}} \nu(s) g(s, \ell) \mu(d s)\right\| \\
& \leq c\left\|\int_{\mathcal{S}} \nu(s) \mu(d s)\right\|=c .
\end{aligned}
$$

Suppose the result holds for $k>1$. Then from Fubini's theorem and (6.42) it follows that

$$
\begin{aligned}
\|\pi(k+1)\|_{1} & =\int_{\mathcal{S}} \int_{\mathcal{S}} \pi(k, s) g(s, \ell) \mu(d s) \mu(d \ell)=\int_{\mathcal{S}} \pi(k, s) \int_{\mathcal{S}} g(s, \ell) \mu(d \ell) \mu(d s) \\
& =\int_{\mathcal{S}} \pi(k, s) \mu(d s)=\|\pi(k)\|_{1}=1,
\end{aligned}
$$


since again $\int_{\mathcal{S}} g(s, \ell) \mu(d \ell)=1$, and from Assumption 6.1.7 and (6.42) one gets that

$$
\begin{aligned}
\|\pi(k+1, \ell)\| & =\left\|\int_{\mathcal{S}} \pi(k, s) g(s, \ell) \mu(d s)\right\| \\
& \leq c\left\|\int_{\mathcal{S}} \pi(k, s) \mu(d s)\right\|=c\|\pi(k)\|_{1}=c .
\end{aligned}
$$

Then indeed $\pi(k) \in \mathbb{H}_{1}^{1+} \cap \mathbb{H}_{\text {sup }}^{1+}$ and $\|\pi(k)\|_{1}=1$, for $k \in \mathbb{N}_{1}$, completing the proof.

Remark 6.2.5. From Proposition A.2.1 there exists an invariant probability density for $\mathcal{G}(\cdot, \cdot)$ denoted by $\pi$ such that $\pi(k) \rightarrow \pi \in \mathbb{H}_{1}^{1+} \cap \mathbb{H}_{\text {sup }}^{1+}$ as $k \rightarrow \infty,\|\pi\|_{1}=1$ and such that it satisfies

$$
\pi(\ell)=\int_{\mathcal{S}} g(s, \ell) \pi(s) \mu(d s)
$$

In this section, as in Section 6.1, it is considered only the family of linear Markov jump filters given by

$$
\left\{\begin{aligned}
\widehat{x}(k+1) & =\widehat{A}(\theta(k)) \widehat{x}(k)+\widehat{B}(\theta(k)) y(k) \\
u(k) & =\widehat{C}(\theta(k)) \widehat{x}(k),
\end{aligned}\right.
$$

where $\widehat{x}(0)=\widehat{x}_{0} \in \mathbb{R}^{n}$ is deterministic and $\widehat{A} \in \mathbb{H}_{\text {sup }}^{n}, \widehat{B} \in \mathbb{H}_{\text {sup }}^{p, n}$ and $\widehat{C} \in \mathbb{H}_{\text {sup }}^{n, m}$. Formally the infinite-horizon optimal filtering problem that is considered in this section is defined below.

Problem 6.2.6. - Infinite-Horizon Filtering Problem: Find $\widehat{A} \in \mathbb{H}_{\text {sup }}^{n}, \widehat{B} \in \mathbb{H}_{\text {sup }}^{p, n}$ and $\widehat{C} \in \mathbb{H}_{\text {sup }}^{n, m}$, and $\widehat{x}_{0} \in \mathbb{R}^{n}$ in (6.45) that minimizes the cost

$$
\limsup _{k \rightarrow \infty} E\left(\|v(k)\|^{2}\right),
$$

where

$$
v(k):=x(k)-\widehat{x}(k) .
$$

\subsubsection{Infinite-Horizon Optimal Filter}

The estimator defined next, already introduced in (6.31), is useful when dealing with the infinite-horizon optimal filtering problem. Set

$$
\begin{aligned}
\widehat{x}_{e}(k+1) & :=A(\theta(k)) \widehat{x}_{e}(k)+B(\theta(k)) u(k)-M(k, \theta(k))\left(y(k)-L(\theta(k)) \widehat{x}_{e}(k)\right) \\
\widehat{x}_{e}(0) & :=E\left(x_{0}\right)=\zeta
\end{aligned}
$$


where $M(k, \ell), k \in \mathbb{N}$, given by $M(k, \ell):=-A(\ell) Y(k, \ell) L(\ell)^{*} U(k, \ell)$, is defined from the filtering $\mathcal{S}$-coupled Riccati difference equations given, for $k \in \mathbb{N}$, by

$$
\begin{aligned}
Y(k+1, \ell) & :=\mathcal{U}(Y(k), \pi(k)) \\
Y(0) & :=V \in \mathbb{H}_{1}^{n+} \cap \mathbb{H}_{\text {sup }}^{n+},
\end{aligned}
$$

that, from (6.8)-(6.12), are given by

$$
\begin{aligned}
U(k, \ell)= & \left(H(\ell) H(\ell)^{*} \pi(k, \ell)+L(\ell) Y(k, \ell) L(\ell)^{*}\right)^{-1}, \\
Y(k+1, \ell)= & \int_{\mathcal{S}}\left(A(s) Y(k, s) A(s)^{*}+G(s) G(s)^{*} \pi(k, s)\right. \\
& \left.-A(s) Y(k, s) L(s)^{*} U(k, s) L(s) Y(k, s) A(s)^{*}\right) g(s, \ell) \mu(d s), \\
Y(0)= & V \in \mathbb{H}_{1}^{n+} \cap \mathbb{H}_{\mathrm{sup}}^{n+} .
\end{aligned}
$$

It follows from Theorem A.2.8 that $M(k) \rightarrow M$ for $k \rightarrow \infty$, where

$$
M(\ell):=\mathcal{M}(Y, \pi)=-A(\ell) Y(\ell) L(\ell)^{*}\left(H(\ell) H(\ell)^{*} \pi(\ell)+L(\ell) Y(\ell) L(\ell)^{*}\right)^{-1}
$$

and $Y$ is the unique solution in $\mathbb{H}_{1}^{n+} \cap \mathbb{H}_{\text {sup }}^{n+}$ of the filtering $\mathcal{S}$-coupled Riccati algebraic equations given by

$$
Y=\mathcal{U}(Y, \pi)=\mathcal{L}(\mathcal{M}(Y, \pi), Y)+\mathcal{I}(\mathcal{M}(Y, \pi), 0, \pi)
$$

where $\pi \in \mathbb{H}_{1}^{1+} \cap \mathbb{H}_{\text {sup }}^{1+}$ is as in Remark 6.2.5. Then set also the following estimator,

$$
\begin{aligned}
\widehat{x}_{e}^{o p}(k+1) & :=A(\theta(k)) \widehat{x}_{e}^{o p}(k)+B(\theta(k)) u(k)-M(\theta(k))\left(y(k)-L(\theta(k)) \widehat{x}_{e}^{o p}(k)\right) \\
\widehat{x}_{e}^{o p}(0) & :=E\left(x_{0}\right)=\zeta .
\end{aligned}
$$

The errors associated with the estimators given in (6.48) and (6.52) are defined by

$$
\begin{aligned}
\widetilde{x}_{e}(k) & :=x(k)-\widehat{x}_{e}(k), \\
\widetilde{x}_{e}^{o p}(k) & :=x(k)-\widehat{x}_{e}^{o p}(k),
\end{aligned}
$$

and from (6.40) and (6.48)-(6.52) one gets that

$$
\widetilde{x}_{e}(k+1)=(A(\theta(k))+M(k, \theta(k)) L(\theta(k))) \widetilde{x}_{e}(k)+(G(\theta(k))+M(k, \theta(k)) H(\theta(k))) w(k),
$$


$\widetilde{x}_{e}^{o p}(k+1)=(A(\theta(k))+M(\theta(k)) L(\theta(k))) \widetilde{x}_{e}^{o p}(k)+(G(\theta(k))+M(\theta(k)) H(\theta(k))) w(k)$.

Notice that (6.52) corresponds to the filter (6.45) with the choice of $\widehat{C} \in \mathbb{H}_{\text {sup }}^{n, m}$ arbitrary, and $\widehat{A}=\widehat{A}^{o p}, \widehat{B}=\widehat{B}^{o p}$ as

$$
\begin{aligned}
& \widehat{A}^{o p}(\theta(k))=A(\theta(k))+M(\theta(k)) L(\theta(k))+B(\theta(k)) \widehat{C}(\theta(k)) \\
& \widehat{B}^{o p}(\theta(k))=-M(\theta(k)) .
\end{aligned}
$$

From the filtering $\mathcal{S}$-coupled Riccati difference equations (6.49) and the filtering $\mathcal{S}$ coupled algebraic Riccati equations (6.51) define the sequence $\left\{Y^{o p}(k) ; k \in \mathbb{N}\right\}$, such that $Y^{o p} \in \mathbb{H}_{1}^{n+} \cap \mathbb{H}_{\text {sup }}^{n+}$ satisfies

$$
\begin{aligned}
Y^{o p}(k+1) & =\mathcal{U}\left(\mathcal{M}(Y, \pi), Y^{o p}(k), \pi(k)\right) \\
Y^{o p}(0) & =Y(0)
\end{aligned}
$$

In the proof of Theorem A.2.8 it is shown that $Y^{o p}(k) \rightarrow Y$ and $Y(k) \rightarrow Y$ as $k \rightarrow \infty$. From Proposition 6.1.18 it follows that

$$
\begin{aligned}
E\left(\left\|\widetilde{x}_{e}(k)\right\|^{2}\right) & =\int_{\mathcal{S}} Y(k, \ell) \mu(d \ell), \\
E\left(\left\|\widetilde{x}_{e}^{o p}(k)\right\|^{2}\right) & =\int_{\mathcal{S}} Y^{o p}(k, \ell) \mu(d \ell) .
\end{aligned}
$$

Moreover it was shown in Proposition 6.1.20 that for $v(k)$ as in (6.47) it follows that

$$
E\left(\|v(k)\|^{2}\right) \geq \int_{\mathcal{S}} \operatorname{tr}(Y(k, s)) \mu(d s) .
$$

The next result presents a solution for the filtering problem posed in Problem 6.2.6. It is based on the unique solution $Y \in \mathbb{H}_{1}^{n+} \cap \mathbb{H}_{\text {sup }}^{n+}$ satisfying (6.51), and $M$ given by (6.50).

Theorem 6.2.7. Suppose that Assumptions 6.1.4 to 6.1 .8 and Assumption 6.2.1 hold, and that $(A, G)$ is stabilizable and $(L, A)$ is detectable. Then an optimal solution for the filtering problem stated in Problem 6.2.6 is given by

$$
\begin{aligned}
& \widehat{A}^{o p}(\ell)=A(\ell)+M(\ell) L(\ell)+B(\ell) \widehat{C}(\ell) \\
& \widehat{B}^{o p}(\ell)=-M(\ell)
\end{aligned}
$$


$\widehat{C} \in \mathbb{H}_{\text {sup }}^{n, m}$ arbitrary, and $\widehat{x}_{0}=\zeta$.

Proof. From Theorem A.2.8, one gets that $Y(k) \rightarrow Y$ as $k \rightarrow \infty$. From the dominated convergence theorem (see Hernández-Lerma and Lasserre, 2003, Section 1.5.2) it follows, taking the limitsup as $k \rightarrow \infty$ in (6.59), that

$$
\limsup _{k \rightarrow \infty} E\left(\|v(k)\|^{2}\right) \geq \lim _{k \rightarrow \infty} \int_{\mathcal{S}} \operatorname{tr}(Y(k, s)) \mu(d s)=\int_{\mathcal{S}} \operatorname{tr}(Y(s)) \mu(d s) .
$$

On the other hand, from (6.58) and

$$
\lim _{k \rightarrow \infty} \int_{\mathcal{S}} \operatorname{tr}\left(Y^{o p}(k, \ell)\right) \mu(d \ell)=\int_{\mathcal{S}} \operatorname{tr}(Y(\ell)) \mu(d \ell)
$$

one gets that the minimum cost $\int_{\mathcal{S}} \operatorname{tr}(Y(s)) \mu(d s)$ is achieved by the filter (6.56)-(6.57).

Note that the result stated in Theorem A.2.8 shows how to obtain the unique solution $Y \in \mathbb{H}_{1}^{n+} \cap \mathbb{H}_{\text {sup }}^{n+}$ recursively from $Y(V, k)$ for any $V \in \mathbb{H}_{1}^{n+} \cap \mathbb{H}_{\text {sup }}^{n+}(Y(V, k) \rightarrow Y$ as $k \rightarrow \infty)$. For practical applications a discretization of the state space may be required since the state space $\mathcal{S}$ is a Borel space, and the recursive equations $Y(V, k)$ are obtained from an integral over $\mathcal{S}$ with respect to $\mu$. Unlike the Kalman filter, the Markovian filter derived in Theorem 6.2.7 is not sample path dependent and thus all the gains can be calculated offline, which is important in applications where the computing power is at premium. Notice also that, since the filtering problem presented in Problem 6.2.6 only takes into account the cost as $k \rightarrow \infty$, it is easy to see that the optimal filter proposed in Theorem 6.2.7 is not unique. 


\section{Chapter 7}

\section{Optimal Control with Partial Information}

This chapter addresses the optimal control considering the partial information case. Recall that in Chapter 5 the LQ optimal control was tackled assuming, for each discrete-time instant $k$, that the state variable $x(k)$ was directly accessible to the controller. This simplifies the problem since in this case the optimal control law is in a state feedback form. On the other hand, in the present chapter it is assumed that the controller has access only to a measurable output variable $y(k)$ and to the jump parameter $\theta(k)$ and, differently from the development done in Chapter 5, the goal is to design a Markov jump controller such that the optimal control law is in an observer-based output feedback form. The finite-horizon LQ optimal control is addressed in Section 7.1 and it is shown that an optimal controller can be obtained from two $\mathcal{S}$-coupled difference Riccati equations, one associated with a filtering problem and the other one to a control problem in which the state variable is fully available. The infinite-horizon optimal control is tackled from a $H_{2}$ optimal control perspective in Section 7.2. The goal in this case is to minimize the $H_{2}$-norm of the closed loop system, that is, it is desired to minimize the limit as time goes to infinity of the expected value of a quadratic cost functional, among the class of stochastic stabilizing Markov jump controllers. It is shown that the $\mathrm{H}_{2}$-norm can be written as the sum of two $\mathrm{H}_{2}$-norms, such that one of them does not depend on the control, and the other one is obtained from the optimal filter for an infinite-horizon filtering problem. This result shows that the optimal controller is obtained from two $\mathcal{S}$-coupled algebraic Riccati equations, one associated with a filtering problem and the other one associated with a control problem in which the state variable is accessible to the controller. Summarizing the results derived in this chapter, similarly to the discrete- 
time linear system case (see Davis and Vinter, 1985, Section 6.3), the optimal controller separates the partial information control problem into two problems, one associated with a filtering problem and the other associated with an optimal control problem. Thus the results obtained in this chapter can be seen as a separation principle for discrete-time MJLS with Markov chain in a general state space. This principle presents a valuable simplification in the control synthesis, since it allows the controller and the filter to be designed independently (Mariton, 1990).

\subsection{Finite-Horizon LQ Optimal Control with Partial Information}

In this section the finite-horizon LQ optimal control problem with partial information is tackled. In Proposition 7.1.9 it is shown that the original partial information problem can be rewritten as a LQ optimal control problem with complete information of the state variable. A solution for this problem is then presented, based on recursive control $\mathcal{S}$ coupled Riccati equations. In Theorem 7.1.13 an optimal controller for the finite-horizon LQ optimal control problem with partial information is derived. This controller is obtained from two $\mathcal{S}$-coupled difference Riccati equations, one associated with a filtering problem and the other one to a control problem in which the state variable is fully available. This result states a separation principle for MJLS with Markov chain in a Borel space $\mathcal{S}$, considering the finite-horizon case.

\subsubsection{Problem Statement}

On the filtered probability space $\left(\Omega, \mathcal{F},\left\{\mathcal{F}_{k}\right\}_{k \in \mathbb{N}}, \mathcal{P}\right)$ consider the following discrete-time Markov jump linear system

$$
\left\{\begin{aligned}
x(k+1) & =A(\theta(k)) x(k)+B(\theta(k)) u(k)+G(\theta(k)) w(k) \\
y(k) & =L(\theta(k)) x(k)+H(\theta(k)) w(k)
\end{aligned}\right.
$$

Recall that the assumptions stated in Section 3.3 hold, in particular $E\left(x_{0}\right):=\zeta$, $E\left(x_{0} x_{0}^{*}\right):=\mathbb{Q}_{0}$, and thus $E\left(\left\|x_{0}-\zeta\right\|^{2}\right)=\operatorname{tr}\left(\mathbb{Q}_{0}-\zeta \zeta^{*}\right)<\infty$. As stated in Remark 3.2.3, in this section the Assumption 3.2.2 is not considered. Assume also the hypotheses stated below. 
Assumption 7.1.1. The noise sequence $\{w(k) ; k \in \mathbb{N}\}$ is a q-dimensional zero mean white noise sequence (that is, $\{w(k) ; k \in \mathbb{N}\}$ is a sequence of independent identically distributed random vectors with $E(w(k))=0$ and $\left.E\left(w(k) w(k)^{\prime}\right)=I\right)$, and is assumed also that $\{w(k) ; k \in \mathbb{N}\}$, the Markov chain $\{\theta(k) ; k \in \mathbb{N}\}$, and the initial state $x_{0}$ are mutually independent.

Assumption 7.1.2. $A \in \widetilde{\mathbb{H}}_{\text {sup }}^{n}, B \in \widetilde{\mathbb{H}}_{\text {sup }}^{m, n}, G \in \widetilde{\mathbb{H}}_{\text {sup }}^{q, n}, L \in \widetilde{\mathbb{H}}_{\text {sup }}^{n, p}, H \in \widehat{\mathbb{H}}_{\text {sup }}^{q, p}$.

Assumption 7.1.3. $G(\ell) H(\ell)^{*}=0$ for all $\ell \in \mathcal{S}$.

Assumption 7.1.4. For some $c \geq 1$ it holds that $g(t, \ell) \leq c$ for all $t, \ell \in \mathcal{S}$.

Assumption 7.1.5. There exist $d>0$ and $\epsilon_{H}(\ell)>0$ such that, for each $k \in \mathbb{N}$, $H(\ell) H(\ell)^{*} \pi(k, \ell) \geq \epsilon_{H}(\ell) I$ and $\frac{\|A(\ell)\|\|L(\ell)\|}{\epsilon_{H}(\ell)} \leq d$ for all $\ell \in \mathcal{S}$.

Notice that the hypotheses assumed in this section are the same assumptions assumed in Section 6.1 for the study of the minimum mean square linear Markov jump filter considering a finite-horizon. Moreover, as in Remark 6.1.1, in this section, for notational simplicity, it is considered that the Markov chain is time-homogeneous and as well as the systems parameters $A, B$, etc. But this assumption is not essential as all the results could be easily adapted to the time-varying case.

In this section consider the sequence $\{\pi(k) ; k \in \mathbb{N}\}$ as defined in (6.2). Also consider a fixed positive $\tau \in \mathbb{N}, \tau>1$, that represents the control problem's time horizon. Recall that the set of nonnegative integers less or equal to $\tau-1$ is denoted by $\mathbb{T}$ and the set of positive integers less or equal to $\tau-1$ is denoted by $\mathbb{T}_{1}$. The class of admissible controls with finite-horizon $\tau$ is defined next.

Definition 7.1.6. - Admissible Controls $\mathcal{U}^{\tau}:$ Choose $\widehat{x}(0)=\widehat{x}_{0} \in \mathbb{R}^{n}$ deterministic and $\widehat{A}(k) \in \widetilde{\mathbb{H}}_{\text {sup }}^{n}, \widehat{B}(k) \in \widetilde{\mathbb{H}}_{\text {sup }}^{p, n}$ and $\widehat{C}(k) \in \widetilde{\mathbb{H}}_{\text {sup }}^{n, m}$ for each $k \in \mathbb{T}$. The class of admissible controllers $u=\{u(k) ; k \in \mathbb{T}\}$ associated with this choice is defined for $u(k)$ obtained from the following Markov jump linear system

$$
\left\{\begin{aligned}
\widehat{x}(k+1) & =\widehat{A}(k, \theta(k)) \widehat{x}(k)+\widehat{B}(k, \theta(k)) y(k) \\
u(k) & =\widehat{C}(k, \theta(k)) \widehat{x}(k) .
\end{aligned}\right.
$$

The class of admissible controls is denoted by $\mathcal{U}^{\tau}$.

Thus the controller can choose the matrices $\widehat{A}(k) \in \widetilde{\mathbb{H}}_{\text {sup }}^{n}, \widehat{B}(k) \in \widetilde{\mathbb{H}}_{\text {sup }}^{p, n}, \widehat{C}(k) \in \mathbb{H}_{\text {sup }}^{n, m}$ and the deterministic initial condition $\widehat{x}(0)$ in $(7.2)$ in order to define the control $u(k)$. 
Notice that the class of admissible controls $\mathcal{U}^{\tau}$ considered in this section has the same definition as the class of admissible filters $\mathcal{F}^{\tau}$ considered in Section 6.1.

Follows from the Assumption 7.1.2, and from $\widehat{A}(k) \in \widetilde{\mathbb{H}}_{\text {sup }}^{n}, \widehat{B}(k) \in \widetilde{\mathbb{H}}_{\text {sup }}^{p, n}$ and $\widehat{C}(k) \in$ $\widetilde{\mathbb{H}}_{\text {sup }}^{n, m}$ that $E\left(\|x(k)\|^{2}\right)<\infty$ and $E\left(\|\widehat{x}(k)\|^{2}\right)<\infty, k \in \mathbb{T}$. From Remark 6.1.11, for any $u$ as in (7.2) it follows that $x(k)$ and $\widehat{x}(k)$ are $\mathcal{F}_{k-1}$-measurable for $k \in \mathbb{N}_{1}$, and, by definition $x_{0}$ is $\mathcal{F}_{0}$-measurable and $\widehat{x}(0)=\widehat{x}_{0}$ is deterministic.

The following hypothesis is related to the quadratic optimal control problem to be defined in the sequel and, as usual in the LQG literature, imposes a strictly positive definite quadratic cost on the control variable $u(k)$.

Assumption 7.1.7. Assume that $C \in \widetilde{\mathbb{H}}_{\text {sup }}^{n, r}, D \in \widetilde{\mathbb{H}}_{\text {sup }}^{m, p}, D(\ell)^{*} D(\ell) \geq \epsilon_{D} I$ for all $\ell \in \mathcal{S}$ and some $\epsilon_{D}>0$, and $S \in \widetilde{\mathbb{H}}_{\text {sup }}^{n+}$.

The quadratic optimal control problem with partial information and finite-horizon $\tau$ that is considered in this section is defined as follows.

Problem 7.1.8. - Finite-Horizon LQ Optimal Control Problem with Partial Information: Find $u \in \mathcal{U}^{\tau}$ that minimizes the $L Q \operatorname{cost} J_{S}^{\tau}\left(x_{0}, \theta_{0}, u\right)$ given by

$$
J_{S}^{\tau}\left(x_{0}, \theta_{0}, u\right):=E\left(\sum_{k=0}^{\tau-1}\|C(\theta(k)) x(k)\|^{2}+\|D(\theta(k)) u(k)\|^{2}+x(\tau)^{*} S(\theta(\tau)) x(\tau)\right)
$$

The finite horizon optimal cost is defined as

$$
J_{S}^{\tau}\left(x_{0}, \theta_{0}\right):=\inf _{u \in \mathcal{U}^{\tau}} J_{S}^{\tau}\left(x_{0}, \theta_{0}, u\right)
$$

\subsubsection{Complete Information Case}

In this section the state estimator defined in (6.31) is again considered. Recall that the estimator (6.31) is based on the filtering $\mathcal{S}$-coupled Riccati difference equations and is given by

$$
\begin{aligned}
\widehat{x}_{e}(k+1) & =A(\theta(k)) \widehat{x}_{e}(k)+B(\theta(k)) u(k)-M(k, \theta(k))\left(y(k)-L(\theta(k)) \widehat{x}_{e}(k)\right) \\
\widehat{x}_{e}(0) & =E\left(x_{0}\right)=\zeta
\end{aligned}
$$

where $M(k, \ell)$ is defined in (6.23). Recall also that, from Remark 6.1.17, $\widehat{x}_{e}(k)$ is $\mathcal{F}_{k-1^{-}}$ measurable for $k \in \mathbb{N}_{1}$ and that the error associated with the estimator stated in (6.31) 
is defined in (6.32) and is given by

$$
\widetilde{x}_{e}(k)=x(k)-\widehat{x}_{e}(k),
$$

such that from (7.1) and (6.31) one gets that

$\widetilde{x}_{e}(k+1)=(A(\theta(k))+M(k, \theta(k)) L(\theta(k))) \widetilde{x}_{e}(k)+(G(\theta(k))+M(k, \theta(k)) H(\theta(k))) w(k)$.

The filtering $\mathcal{S}$-coupled Riccati difference equations defined in (6.21) are reproduced below for convenience.

Recall the definition of the operators $\mathcal{L}, \mathcal{I}, \mathcal{U}$ and $\mathcal{M}$ stated in (6.8)-(6.12) and the definition of the operators $\widetilde{\mathcal{L}}, \widetilde{\mathcal{I}}$ and $\widetilde{\mathcal{U}}$ stated in (6.13)-(6.16). For $\ell \in \mathcal{S}$, the filtering $\mathcal{S}$-coupled Riccati difference equations and the filter gains $M(k) \in \mathbb{H}_{\text {sup }}^{p, n}$ are given by

$$
\begin{aligned}
Y(k+1) & =\mathcal{U}(Y(k), \pi(k)), \quad \text { for } k \in \mathbb{T}_{1}, \\
Y(1) & =\widetilde{\mathcal{U}}(Y(0), \pi(0)), \\
M(k, \ell) & =\mathcal{M}(Y(k), \pi(k)), \quad \text { for } k \in \mathbb{T},
\end{aligned}
$$

where $Y(0) \in \mathbb{H}_{\text {sup }}^{n+}$ is given by

$$
Y(0)=\left(\mathbb{Q}_{0}-\zeta \zeta^{\prime}\right)=E\left(\left(x_{0}-\zeta\right)\left(x_{0}-\zeta\right)^{\prime}\right)
$$

In the next proposition the original LQ optimal control problem with partial information (Problem 7.1.8) is written as a LQ optimal control problem with complete information of the state variable.

Proposition 7.1.9. Minimizing $J_{S}^{\tau}\left(x_{0}, \theta_{0}, u\right)$, as stated in Problem 7.1 .8 , is equivalent to minimizing $J_{e}\left(\zeta, \theta_{0}, u\right)$ given by

$$
J_{e}\left(\zeta, \theta_{0}, u\right):=E\left(\sum_{k=0}^{\tau-1}\left\|C(\theta(k)) \widehat{x}_{e}(k)\right\|^{2}+\|D(\theta(k)) u(k)\|^{2}+\widehat{x}_{e}(\tau)^{*} S(\theta(\tau)) \widehat{x}_{e}(\tau)\right)
$$

subject to

$$
\left\{\begin{aligned}
\widehat{x}_{e}(k+1) & =A(\theta(k)) \widehat{x}_{e}(k)+B(\theta(k)) u(k)-M(k, \theta(k)) \xi(k) \\
\widehat{x}_{e}(0) & =E\left(x_{0}\right)=\zeta,
\end{aligned}\right.
$$


where

$$
\xi(k)=y(k)-L(\theta(k)) \widehat{x}_{e}(k)=L(\theta(k)) \widetilde{x}_{e}(k)+H(\theta(k)) w(k)
$$

and $u \in \mathcal{U}^{\tau}$ is as in (7.2). The optimal cost is given by

$$
J_{e}\left(\zeta, \theta_{0}\right):=\inf _{u \in \mathcal{U}^{\tau}} J_{e}\left(\zeta, \theta_{0}, u\right)
$$

Proof. Noticing that $x(k)=\widetilde{x}_{e}(k)+\widehat{x}_{e}(k)$ and from the fact that $x(k), \widehat{x}_{e}(k)$ are $\mathcal{F}_{k-1^{-}}$ measurable (see Remarks 6.1.11 and 6.1.17), one gets for $k \in \mathbb{T}_{1}$,

$$
\begin{aligned}
E\left(\|C(\theta(k)) x(k)\|^{2}\right)=E\left(E\left(x(k)^{*} C(\theta(k))^{*} C(\theta(k)) x(k) \mid \mathcal{F}_{k-1}\right)\right) \\
=E\left(x(k)^{*} E\left(C(\theta(k))^{*} C(\theta(k)) \mid \mathcal{F}_{k-1}\right) x(k)\right) \\
=E\left(x(k)^{*} \int_{\mathcal{S}} C(s)^{*} C(s) g(\theta(k-1), s) \mu(d s) x(k)\right) \\
=\int_{\mathcal{S}} \operatorname{tr}\left(C(s) E\left(x(k) x(k)^{*} g(\theta(k-1), s)\right) C(s)^{*}\right) \mu(d s) \\
=\int_{\mathcal{S}} \operatorname{tr}\left(C(s) E\left(\left(\widetilde{x}_{e}(k)+\widehat{x}_{e}(k)\right)\left(\widetilde{x}_{e}(k)+\widehat{x}_{e}(k)\right)^{*} g(\theta(k-1), s)\right) C(s)^{*}\right) \mu(d s) .
\end{aligned}
$$

From Proposition 6.1.19 one has that $E\left(\widetilde{x}_{e}(k) \widehat{x}_{e}(k)^{*} g(\theta(k-1), \ell)\right)=0$ and from Proposition 6.1.18 that $Y(k, s)=E\left(\widetilde{x}_{e}(k) \widetilde{x}_{e}(k)^{*} g(\theta(k-1), s)\right)$, so that

$$
E\left(\left(\widetilde{x}_{e}(k)+\widehat{x}_{e}(k)\right)\left(\widetilde{x}_{e}(k)+\widehat{x}_{e}(k)\right)^{*} g(\theta(k-1), s)\right)=Y(k, s)+E\left(\widehat{x}_{e}(k) \widehat{x}_{e}(k)^{*} g(\theta(k-1), s)\right) .
$$

Combining (7.10) and (7.11) it follows that

$$
E\left(\|C(\theta(k)) x(k)\|^{2}\right)=E\left(\left\|C(\theta(k)) \widehat{x}_{e}(k)\right\|^{2}\right)+\int_{\mathcal{S}} \operatorname{tr}\left(C(s) Y(k, s) C(s)^{*}\right) \mu(d s),
$$

and for $k=0$ one gets that

$$
E\left(\left\|C\left(\theta_{0}\right) x_{0}\right\|^{2}\right)=E\left(\left\|C\left(\theta_{0}\right) \widehat{x}_{e}(0)\right\|^{2}\right)+\int_{\mathcal{S}} \operatorname{tr}\left(C(s) Y(0, s) C(s)^{*}\right) \widehat{\mu}_{\mathcal{S}}(d s) .
$$

Similarly for $k=\tau$ it follows that

$$
E\left(x(\tau)^{*} S(\theta(\tau)) x(\tau)\right)=E\left(\widehat{x}_{e}(\tau)^{*} S(\theta(\tau)) \widehat{x}_{e}(\tau)\right)+\int_{\mathcal{S}} \operatorname{tr}(S(s) Y(\tau, s)) \mu(d s)
$$

Combining (7.12)-(7.14) and recalling that $\widehat{x}_{e}(0)=\zeta$ one gets from (7.3) that

$$
J_{S}^{\tau}\left(x_{0}, \theta_{0}, u\right)=J_{e}\left(\zeta, \theta_{0}, u\right)+\Psi\left(x_{0}, \theta_{0}\right)
$$


where

$$
J_{e}\left(\zeta, \theta_{0}, u\right)=\sum_{k=0}^{\tau-1} E\left(\left\|C(\theta(k)) \widehat{x}_{e}(k)\right\|^{2}+\|D(\theta(k)) u(k)\|^{2}\right)+E\left(\widehat{x}_{e}(\tau)^{*} S(\theta(\tau)) \widehat{x}_{e}(\tau)\right)
$$

and

$$
\begin{aligned}
\Psi\left(x_{0}, \theta_{0}\right):=\int_{\mathcal{S}} & \operatorname{tr}\left(C(s) Y(0) C(s)^{*}\right) \widehat{\mu}_{\mathcal{S}}(d s)+\sum_{k=1}^{\tau-1} \int_{\mathcal{S}} \operatorname{tr}\left(C(s) Y(k, s) C(s)^{*}\right) \mu(d s) \\
& +\int_{\mathcal{S}} \operatorname{tr}(S(s) Y(\tau, s)) \mu(d s) .
\end{aligned}
$$

From (7.16) notice that $\Psi\left(x_{0}, \theta_{0}\right)$ does not depend on $u$ and thus minimizing $J_{S}^{\tau}\left(x_{0}, \theta_{0}, u\right)$ is equivalent to minimizing $J_{e}\left(\zeta, \theta_{0}, u\right)$ subject to

$$
\left\{\begin{aligned}
\widehat{x}_{e}(k+1) & =A(\theta(k)) \widehat{x}_{e}(k)+B(\theta(k)) u(k)-M(k, \theta(k)) \xi(k) \\
\widehat{x}_{e}(0) & =E\left(x_{0}\right)=\zeta,
\end{aligned}\right.
$$

where

$$
\xi(k)=y(k)-L(\theta(k)) \widehat{x}_{e}(k)=L(\theta(k)) \widetilde{x}_{e}(k)+H(\theta(k)) w(k)
$$

and $u \in \mathcal{U}^{\tau}$ is given by (7.2). Setting

$$
J_{e}\left(\zeta, \theta_{0}\right)=\inf _{u \in \mathcal{U}^{\tau}} J_{e}\left(\zeta, \theta_{0}, u\right)
$$

it follows from $(7.15)$ that $J_{S}^{\tau}\left(x_{0}, \theta_{0}\right)=J_{e}\left(\zeta, \theta_{0}\right)+\Psi\left(x_{0}, \theta_{0}\right)$, completing the proof.

The problem posed in (7.9) can be considered a complete information optimal control case since that the state variable $\widehat{x}_{e}(k)$ and the jump variable $\theta(k)$ are available at each discrete-time instant $k$ for the controller. The solution for this problem is obtained from the control $\mathcal{S}$-coupled Riccati difference equations defined in (5.4) and reproduced below.

Recall the definition of the operators $\mathcal{Q}, \mathcal{K}$, and $\mathcal{R}$ stated in Definition 5.1.4. For $k=\tau-1, \ldots, 0$, the control $\mathcal{S}$-coupled Riccati difference equations and the feedback gains $K(k) \in \mathbb{H}_{\text {sup }}^{n, m}$ are given by

$$
\begin{aligned}
X^{\tau}(k) & =\mathcal{R}\left(X^{\tau}(k+1)\right) \\
X^{\tau}(\tau) & =S, \\
K^{\tau}(k) & =-\mathcal{K}\left(X^{\tau}(k+1)\right) .
\end{aligned}
$$


Remark 7.1.10. As pointed out in Proposition 5.1.5, $\mathcal{E}, \mathcal{Q}, \mathcal{K}$ and $\mathcal{R}$ map $\mathbb{H}_{\text {sup }}^{\text {n+ }}$ into respectively $\mathbb{H}_{\text {sup }}^{n+}, \mathbb{H}_{\text {sup }}^{m+}, \mathbb{H}_{\text {sup }}^{n, m}$ and $\mathbb{H}_{\text {sup }}^{n+}$, and $\mathcal{Q}(Z)^{-1} \in \mathbb{H}_{\text {sup }}^{m+}$ for $Z \in \mathbb{H}_{\text {sup }}^{n+}$.

From the $\mathcal{S}$-coupled Riccati difference equations, for $k=\tau-1, \ldots, 0$, define the functions

$$
\begin{aligned}
\mathcal{W}(k, \ell, x): & x^{*} X^{\tau}(k, \ell) x+\alpha(k), \\
\alpha(k):= & \sum_{j=k}^{\tau-1} \delta(j) \\
\delta(j):= & \int_{\mathcal{S}} \operatorname{tr}\left(\mathcal { E } ( X ^ { \tau } ( j + 1 ) ) ( s ) M ( j , s ) \left(L(s) Y(j, s) L(s)^{*}\right.\right. \\
& \left.\left.+H(s) H(s)^{*} \pi(j, s)\right) M(j, s)^{*}\right) \mu(d s), \quad j \geq 1, \\
\delta(0):= & \int_{\mathcal{S}} \operatorname{tr}\left(\mathcal{E}\left(X^{\tau}(1)\right)(s) M(0, s)\left(L(s) Y(0) L(s)^{*}+H(s) H(s)^{*}\right) M(0, s)^{*}\right) \widehat{\mu}_{\mathcal{S}}(d s) .
\end{aligned}
$$

Proposition 7.1.11. For $\widehat{x}_{e}(k)$ given by (7.7), $u(k)$ by (7.2) and $\xi(k)$ by (7.8) it holds that

$$
E\left(\left(A(\theta(k)) \widehat{x}_{e}(k)+B(\theta(k)) u(k)\right)^{*} X^{\tau}(k+1, \theta(k+1)) M(k, \theta(k)) \xi(k)\right)=0 .
$$

Proof. From Assumption 7.1.1, $w(k)$ is independent of $\theta(k), \theta(k+1)$ and $\widehat{x}_{e}(k)$, and therefore since $E(w(k))=0$,

$$
\begin{aligned}
& E\left(\left(A(\theta(k)) \widehat{x}_{e}(k)+B(\theta(k)) u(k)\right)^{*} X^{\tau}(k+1, \theta(k+1)) M(k, \theta(k)) H(\theta(k)) w(k)\right) \\
& \quad=E\left(\left(A(\theta(k)) \widehat{x}_{e}(k)+B(\theta(k)) u(k)\right)^{*} X^{\tau}(k+1, \theta(k+1)) M(k, \theta(k)) H(\theta(k))\right) E(w(k)) \\
& \quad=0
\end{aligned}
$$

and thus from (7.8),

$$
\begin{aligned}
& E\left(\left(A(\theta(k)) \widehat{x}_{e}(k)+B(\theta(k)) u(k)\right)^{*} X^{\tau}(k+1, \theta(k+1)) M(k, \theta(k)) \xi(k)\right) \\
& =E\left(\left(A(\theta(k)) \widehat{x}_{e}(k)+B(\theta(k)) u(k)\right)^{*} X^{\tau}(k+1, \theta(k+1)) M(k, \theta(k)) L(\theta(k)) \widetilde{x}_{e}(k)\right) .
\end{aligned}
$$

Consider $k \geq 1$. Since $\widehat{x}_{e}(k), \widetilde{x}_{e}(k), \widehat{x}(k)$ are $\mathcal{F}_{k-1}$ measurable (and thus also $\mathcal{F}_{k}$ measur- 
able), $u(k)=\widehat{C}(k, \theta(k)) \widehat{x}(k)$, one gets that

$$
\begin{aligned}
& E\left(\left(A(\theta(k)) \widehat{x}_{e}(k)+B(\theta(k)) u(k)\right)^{*} X^{\tau}(k+1, \theta(k+1)) M(k, \theta(k)) L(\theta(k)) \widetilde{x}_{e}(k)\right) \\
& =E\left(E\left(\left(A(\theta(k)) \widehat{x}_{e}(k)+B(\theta(k)) u(k)\right)^{*} X^{\tau}(k+1, \theta(k+1)) M(k, \theta(k)) L(\theta(k)) \widetilde{x}_{e}(k) \mid \mathcal{F}_{k}\right)\right) \\
& =E\left(\left(A(\theta(k)) \widehat{x}_{e}(k)+B(\theta(k)) u(k)\right)^{*} \mathcal{E}\left(X^{\tau}(k+1)\right)(\theta(k)) M(k, \theta(k)) L(\theta(k)) \widetilde{x}_{e}(k)\right),
\end{aligned}
$$

and

$$
\begin{aligned}
& E\left((B(\theta(k)) u(k))^{*} \mathcal{E}\left(X^{\tau}(k+1)\right)(\theta(k)) M(k, \theta(k)) L(\theta(k)) \widetilde{x}_{e}(k)\right) \\
& =E\left(E\left(\operatorname{tr}\left(\widehat{C}(k, \theta(k))^{*} B(\theta(k))^{*} \mathcal{E}\left(X^{\tau}(k+1)\right)(\theta(k)) M(k, \theta(k)) L(\theta(k)) \widetilde{x}_{e}(k) \widehat{x}(k)^{*}\right) \mid \mathcal{F}_{k-1}\right)\right) \\
& =\int_{\mathcal{S}} \operatorname{tr}\left(\widehat{C}(k, s)^{*} B(s)^{*} \mathcal{E}\left(X^{\tau}(k+1)\right)(s) M(k, s) L(s) E\left(\widetilde{x}_{e}(k) \widehat{x}(k)^{*} g(\theta(k-1), s)\right)\right) \mu(d s) \\
& =0
\end{aligned}
$$

where the last equality follows from (6.35). Similar reasoning using (6.36) shows that

$$
E\left(\left(A(\theta(k)) \widehat{x}_{e}(k)\right)^{*} \mathcal{E}\left(X^{\tau}(k+1)\right)(\theta(k)) M(k, \theta(k)) L(\theta(k)) \widetilde{x}_{e}(k)\right)=0
$$

Combining (7.19)-(7.22) one gets (7.18) for $k=1, \ldots, \tau$. The case $k=0$ can be obtained in a similar manner after noticing that $E\left(\widetilde{x}_{e}(0)\right)=0$ and $\widehat{x}_{e}(0)=\zeta, \widehat{x}(0)$ is deterministic.

A solution to the problem posed in (7.9) is presented next.

Theorem 7.1.12. An optimal control law $u^{o p}$ for the problem posed in (7.9) is given by $u^{o p}=\left\{u^{o p}(k) ; k \in \mathbb{T}\right\}$, where

$$
u^{o p}(k)=K(k, \theta(k)) \widehat{x}_{e}(k) .
$$

The optimal cost is given by

$$
J_{e}\left(\zeta, \theta_{0}\right)=\zeta^{*}\left(\int_{\mathcal{S}} X^{\tau}(0, t) \widehat{\mu}_{\mathcal{S}}(d t)\right) \zeta+\alpha(0) .
$$

Proof. In what follows set

$$
\|z(k)\|^{2}=\left\|C(\theta(k)) \widehat{x}_{e}(k)\right\|^{2}+\|D(\theta(k)) u(k)\|^{2} .
$$


Using similar arguments as in the proof of Proposition 7.1.11 and from (7.18) one gets that

$$
\begin{aligned}
E\left(\widehat{x}_{e}(k+1)^{*} X^{\tau}(k+1, \theta(k+1)) \widehat{x}_{e}(k+1)\right) \\
=E\left(\widehat{x}_{e}(k+1)^{*} \mathcal{E}\left(X^{\tau}(k+1)\right)(\theta(k)) \widehat{x}_{e}(k+1)\right) \\
=E\left(( A ( \theta ( k ) ) \widehat { x } _ { e } ( k ) + B ( \theta ( k ) ) u ( k ) ) ^ { * } \mathcal { E } ( X ^ { \tau } ( k + 1 ) ) ( \theta ( k ) ) \left(A(\theta(k)) \widehat{x}_{e}(k)\right.\right. \\
\left.\quad+B(\theta(k)) u(k)))+E\left((M(k, \theta(k)) \xi(k))^{*} \mathcal{E}\left(X^{\tau}(k+1)\right)(\theta(k))(M(k, \theta(k)) \xi(k))\right)\right) .
\end{aligned}
$$

Following a similar approach as in Theorem 5.1.6 and in Theorem 1 in Costa and Tuesta (2003), one gets from (7.17) that

$$
\begin{aligned}
& E\left(\left(A(\theta(k)) \widehat{x}_{e}(k)+B(\theta(k)) u(k)\right)^{*} \mathcal{E}\left(X^{\tau}(k+1)\right)(\theta(k))\right.\left.\left(A(\theta(k)) \widehat{x}_{e}(k)+B(\theta(k)) u(k)\right)-x(k)^{*} X^{\tau}(k, \theta(k)) x(k)\right) \\
&=-E\left(\|z(k)\|^{2}\right)+E\left(\left\|\mathcal{Q}\left(X^{\tau}(k)\right)(\theta(k))^{1 / 2}\left(u(k)-F(k, \theta(k)) \widehat{x}_{e}(k)\right)\right\|^{2}\right),
\end{aligned}
$$

and that

$$
\begin{aligned}
E & \left((M(k, \theta(k)) \xi(k))^{*} \mathcal{E}\left(X^{\tau}(k+1)\right)(\theta(k)) M(k, \theta(k)) \xi(k)\right) \\
& =E\left(\operatorname{tr}\left(M(k, \theta(k))^{*} \mathcal{E}\left(X^{\tau}(k+1)\right)(\theta(k)) M\left(k, \theta(k) \xi(k) \xi(k)^{*}\right)\right)\right) \\
& =\delta(k) .
\end{aligned}
$$

From (7.25)-(7.27) it follows that

$$
\begin{aligned}
& E\left(\mathcal{W}\left(k+1, \theta(k+1), \widehat{x}_{e}(k+1)\right)-\mathcal{W}\left(k, \theta(k), \widehat{x}_{e}(k)\right)\right) \\
& \quad=-\|z(k)\|^{2}+E\left(\left\|\mathcal{Q}\left(X^{\tau}(k)\right)(\theta(k))^{1 / 2}\left(u(k)-F(k, \theta(k)) \widehat{x}_{e}(k)\right)\right\|^{2}\right) .
\end{aligned}
$$

Taking the sum in (7.28) from $k=0$ to $\tau-1$, and recalling that

$$
\mathcal{W}\left(\tau, \theta(\tau), \widehat{x}_{e}(\tau)\right)=\widehat{x}_{e}(\tau)^{*} S(\theta(\tau)) \widehat{x}_{e}(\tau)
$$

one gets that

$$
J_{e}\left(\zeta, \theta_{0}, u\right)=E\left(\sum_{k=0}^{\tau-1}\|z(k)\|^{2}+\widehat{x}_{e}(\tau)^{*} S(\theta(\tau)) \widehat{x}_{e}(\tau)\right)
$$




$$
=E\left(\mathcal{W}\left(0, \theta_{0}, \zeta\right)\right)+\sum_{k=0}^{\tau-1} E\left(\left\|\mathcal{Q}\left(X^{\tau}(k)\right)(\theta(k))^{1 / 2}\left(u(k)-F(k, \theta(k)) \widehat{x}_{e}(k)\right)\right\|^{2}\right)
$$

Taking the minimum over $u \in \mathcal{U}^{\tau}$ in (7.29) one obtains that the optimal control law is given by (7.23), so that the second term on the right hand side of (7.29) equals to zero, yielding (7.24).

\subsubsection{A Separation Principle}

The main result of this section is presented below. It combines the previous results to address the finite-horizon LQ optimal control problem with partial information stated in Problem 7.1.8.

Theorem 7.1.13. An optimal solution for the Problem 7.1 .8 is obtained from the filtering $\mathcal{S}$-coupled Riccati difference equations (7.5) and the control $\mathcal{S}$-coupled Riccati difference equations (7.17). The gains $M$ given by (7.5) and $K$ given by (7.17) lead to the following optimal solution, for $\ell \in \mathcal{S}$,

$$
\begin{aligned}
& \widehat{A}^{o p}(k, \ell)=A(\ell)+M(k, \ell) L(\ell)+B(\ell) K(k, \ell), \\
& \widehat{B}^{o p}(k, \ell)=-M(k, \ell), \\
& \widehat{C}^{o p}(k, \ell)=K(k, \ell),
\end{aligned}
$$

and the optimal cost is

$$
J\left(x_{0}, \theta_{0}, u\right)=\zeta^{*}\left(\int_{\mathcal{S}} X^{\tau}(0, t) \widehat{\mu}_{\mathcal{S}}(d t)\right) \zeta+\alpha(0)+\Psi\left(x_{0}, \theta_{0}\right)
$$

Proof. From Proposition (6.1.14) one has that $M(k) \in \mathbb{H}_{\text {sup }}^{p, n}$ and from Proposition 5.1.5 it follows that $K(k) \in \mathbb{H}_{\text {sup }}^{n, m}$. From these it follows that $\widehat{A}^{o p}(k) \in \mathbb{H}_{\text {sup }}^{n}, \widehat{B}^{o p}(k) \in \mathbb{H}_{\text {sup }}^{p, n}$ and $\widehat{C}^{o p}(k) \in \mathbb{H}_{\text {sup }}^{n, m}$. From (7.15)-(7.16) together with (7.24) yield (7.30). Considering (7.7)-(7.8) with the optimal control law $u^{o p}$ in (7.23) one gets that

$$
\begin{aligned}
\widehat{x}_{e}(k+1) & =\widehat{A}^{o p}(k, \theta(k)) \widehat{x}_{e}(k)+\widehat{B}^{o p}(k, \theta(k)) y(k), \\
u^{o p}(k) & =F(k, \theta(k)) \widehat{x}_{e}(k)=\widehat{C}^{o p}(k, \theta(k)) \widehat{x}_{e}(k),
\end{aligned}
$$

so that from Theorem 7.1.12 it follows that $u^{o p} \in \mathcal{U}^{\tau}$ is an optimal solution for the Problem 7.1.8. 


\subsection{Infinite-Horizon $\mathrm{H}_{2}$ Optimal Control}

The infinite-horizon optimal control with partial information is tackled in this section. In Theorem 7.2.18 it is shown that the $H_{2}$-norm can be written as the sum of two $H_{2}$-norms, such that one of them does not depend on the control, and the other one is obtained from the optimal filter for an infinite-horizon filtering problem. That is, the optimal controller is obtained from two $\mathcal{S}$-coupled algebraic Riccati equations, one associated with a filtering problem and the other one associated with a control problem with complete observations. This result can be seen as a separation principle for the infinite-horizon case.

\subsection{1 $\mathrm{H}_{2}$ Optimal Control Problem}

On the filtered probability space $\left(\Omega, \mathcal{F},\left\{\mathcal{F}_{k}\right\}_{k \in \mathbb{N}}, \mathcal{P}\right)$, consider the following MJLS $\mathcal{G}_{G}$,

$$
\mathcal{G}_{G}=\left\{\begin{aligned}
x(k+1) & =A(\theta(k)) x(k)+B(\theta(k)) u(k)+G(\theta(k)) w(k) \\
y(k) & =L(\theta(k)) x(k)+H(\theta(k)) w(k) \\
z(k) & =C(\theta(k)) x(k)+D(\theta(k)) u(k),
\end{aligned}\right.
$$

with initial condition $\left(x_{0}, \theta_{0}\right)$ having distribution $\widehat{\mu}$, for some probability measure $\widehat{\mu}$ on $\mathbb{C}^{n} \times \mathcal{S}$, such that $E\left(x_{0}\right):=\zeta, E\left(x_{0} x_{0}^{*}\right):=\mathbb{Q}_{0}$, and $x_{0}, \theta_{0}$ are mutually independent. Thus, $E\left(\left\|x_{0}-\zeta\right\|^{2}\right)=\operatorname{tr}\left(\mathbb{Q}_{0}-\zeta \zeta^{*}\right)<\infty$. The initial probability measure on $\mathcal{S}$ for $\theta_{0}$ is denoted $\widehat{\mu}_{\mathcal{S}}$, and it is assumed that $\widehat{\mu}_{\mathcal{S}} \ll \mu$ as in Assumption 3.2.2, such that there exists $\nu \in \mathbb{H}_{1}^{1+}$. Assume also that:

Assumption 7.2.1. $A \in \mathbb{H}_{\text {sup }}^{n}, B \in \mathbb{H}_{\text {sup }}^{m, n}, G \in \mathbb{H}_{\text {sup }}^{q, n}, L \in \mathbb{H}_{\text {sup }}^{n, p}, H \in \mathbb{H}_{\text {sup }}^{q, p}, C \in \mathbb{H}_{\text {sup }}^{n, r}$, $D \in \mathbb{H}_{\text {sup }}^{m, r}$.

Assumption 7.2.2. $G(\ell) H(\ell)^{*}=0$ for all $\ell \in \mathcal{S}$.

Assumption 7.2.3. $C(\ell)^{*} D(\ell)=0$ and $D(\ell)^{*} D(\ell) \geq \epsilon_{D} I$ for all $\ell \in \mathcal{S}$ and some $\epsilon_{D}>0$.

Assumption 7.2.4. For some $c \geq 1$ it holds that $g(t, \ell) \leq c$ for all $t, \ell \in \mathcal{S}$.

Assumption 7.2.5. There exist $d>0$ and $\epsilon_{H}(\ell)>0$ such that, for each $k \in \mathbb{N}$, $H(\ell) H(\ell)^{*} \pi(k, \ell) \geq \epsilon_{H}(\ell) I$ and $\frac{\|A(\ell)\|\|L(\ell)\|}{\epsilon_{H}(\ell)} \leq d$ for all $\ell \in \mathcal{S}$.

Assumption 7.2.6. $\{\theta(k) ; k \in \mathbb{N}\}$ is uniformly ergodic.

Consider the sequence $\{\pi(k) ; k \in \mathbb{N}\}$ as defined in (6.41). Thus, for $k \in \mathbb{N}_{1}, \pi(k) \in$ $\mathbb{H}_{1}^{1+} \cap \mathbb{H}_{\text {sup }}^{1+}$. Moreover, there exists an invariant probability density for $\mathcal{G}(\cdot, \cdot)$ denoted 
by $\pi$ such that $\pi(k) \rightarrow \pi \in \mathbb{H}_{1}^{1+} \cap \mathbb{H}_{\text {sup }}^{1+}$ as $k \rightarrow \infty,\|\pi\|_{1}=1$ and such that it satisfies $\pi(\ell)=\int_{\mathcal{S}} g(s, \ell) \pi(s) \mu(d s)$ (see Proposition A.2.1).

In this section it is again considered the design of Markov jump controllers $\mathcal{G}_{K}$ for system (7.31) given by

$$
\mathcal{G}_{K}=\left\{\begin{aligned}
\widehat{x}(k+1) & =\widehat{A}(\theta(k)) \widehat{x}(k)+\widehat{B}(\theta(k)) y(k) \\
u(k) & =\widehat{C}(\theta(k)) \widehat{x}(k),
\end{aligned}\right.
$$

with $\widehat{x}(0)=\widehat{x}_{0}$ deterministic and $\widehat{A}(k) \in \mathbb{H}_{\text {sup }}^{n}, \widehat{B}(k) \in \mathbb{H}_{\text {sup }}^{p, n}$ and $\widehat{C}(k) \in \mathbb{H}_{\text {sup }}^{n, m}$ for each $k \in \mathbb{N}$. From (7.31) and (7.32) it follows that the closed loop system is

$$
\left\{\begin{aligned}
{\left[\begin{array}{l}
x(k+1) \\
\widehat{x}(k+1)
\end{array}\right]=} & {\left[\begin{array}{cc}
A(\theta(k)) & B(\theta(k)) \widehat{C}(\theta(k)) \\
\widehat{B}(\theta(k)) L(\theta(k)) & \widehat{A}(\theta(k))
\end{array}\right]\left[\begin{array}{l}
x(k) \\
\widehat{x}(k)
\end{array}\right] } \\
& +\left[\begin{array}{c}
G(\theta(k)) \\
\widehat{B}(\theta(k)) H(\theta(k))
\end{array}\right] w(k) \\
z(k)= & {\left[\begin{array}{ll}
C(\theta(k)) & D(\theta(k)) \widehat{C}(\theta(k))
\end{array}\right]\left[\begin{array}{c}
x(k) \\
\widehat{x}(k)
\end{array}\right] . }
\end{aligned}\right.
$$

Defining

$$
\begin{aligned}
\Upsilon(\theta(k)) & :=\left[\begin{array}{cc}
A(\theta(k)) & B(\theta(k)) \widehat{C}(\theta(k)) \\
\widehat{B}(\theta(k)) L(\theta(k)) & \widehat{A}(\theta(k))
\end{array}\right], \\
\Psi(\theta(k)) & :=\left[\begin{array}{c}
G(\theta(k)) \\
\widehat{B}(\theta(k)) H(\theta(k))
\end{array}\right], \\
\Phi(\theta(k)) & :=\left[\begin{array}{cc}
C(\theta(k)) & D(\theta(k)) \widehat{C}(\theta(k))]
\end{array}\right] \\
\mathbf{v}(k) & :=\left[\begin{array}{c}
x(k) \\
\widehat{x}(k)
\end{array}\right]
\end{aligned}
$$

one gets that (7.33) can be rewritten as

$$
\mathcal{G}_{c l}=\left\{\begin{aligned}
\mathbf{v}(k+1) & =\Upsilon(\theta(k)) \mathbf{v}(k)+\Psi(\theta(k)) w(k) \\
z(k) & =\Phi(\theta(k)) \mathbf{v}(k) .
\end{aligned}\right.
$$

The class of admissible controls $\mathcal{U}$ is defined next. 
Definition 7.2.7. - Admissible Controls $\mathcal{U}$ : The controls $u=\{u(k) ; k \in \mathbb{N}\}$ obtained from (7.32) such that the closed loop system $\mathcal{G}_{c l}$ (7.38) is stochastically stable is referred to as the class of admissible controls and is denoted by $\mathcal{U}$.

Next a remark for the model (7.31) is presented.

Remark 7.2.8. For any $u \in \mathcal{U}$ it holds that $x(k)$ and $\widehat{x}(k)$ are $\mathcal{F}_{k-1}$-measurable for $k \in \mathbb{N}_{1}$. Indeed it is easy to see from (7.31) and (7.32) that $x(k)$ and $\widehat{x}(k)$ can be written in function of $x_{0}$ and $w(j), \theta(j)$, for $j=0, \ldots, k-1$. Moreover by definition it holds that $x_{0}$ is $\mathcal{F}_{0}$-measurable and $\widehat{x}(0)=\widehat{x}_{0}$ is deterministic. From (7.37) it is immediate that $\mathbf{v}(k)$ is also $\mathcal{F}_{k-1}$-measurable for $k \in \mathbb{N}$.

Consider the closed loop system (7.38). The $H_{2}$ optimal control problem with partial information that is considered in this section is defined below. Its definition follows from the $H_{2}$ optimal control problem for MJLS with Markov chain taking values in a finite state space (see Costa et al., 2005c, Section 6.3), adapted for the general state space case.

Problem 7.2.9. - $H_{2}$ Optimal Control Problem with Partial Information: Find $u \in \mathcal{U}$ that minimizes the $H_{2}$-norm of the closed loop system $\mathcal{G}_{c l}$, denoted by $\left\|\mathcal{G}_{G}\right\|_{2}$, given by (7.38) with $\mathbf{v}(0)=0$, defined as

$$
\left\|\mathcal{G}_{G}\right\|_{2}^{2}=\sum_{s=1}^{q}\left\|z_{s}\right\|_{2}^{2},
$$

where

$$
\left\|z_{s}\right\|_{2}^{2}=\sum_{k=1}^{\infty} E\left(\left\|z_{s}(k)\right\|^{2}\right)
$$

and $z_{s}=\left(z_{s}(0), z_{s}(1), \ldots\right)$ represents the output sequence when:

(a) The input sequence $\{w(k) ; k \in \mathbb{N}\}$ is given by $w=(w(0), w(1), \ldots), w(0)=e_{s}$, $w(k)=0$ for each $k>0$, with $e_{s} \in \mathbb{R}^{q}$ the unitary vector formed by 1 at the $s^{\text {th }}$ position, 0 elsewhere, and

(b) $\mathcal{P}\left(\theta_{0} \in B\right)=\int_{B} \pi(\ell) \mu(d \ell)$, for $B \in \mathcal{B}(\mathcal{S})$.

Notice that assuming $\mathcal{P}\left(\theta_{0} \in B\right)=\int_{B} \pi(\ell) \mu(d \ell)$, for $B \in \mathcal{B}(\mathcal{S})$, as in hypothesis $(b)$ in Problem 7.2.9, from Proposition 6.2.3 it follows that $\mathcal{P}(\theta(k) \in B)=\int_{B} \pi(\ell) \mu(d \ell)$, for $k \in \mathbb{N}$. 
Consider the following $\mathcal{S}$-coupled algebraic equations, for $s, \ell \in \mathcal{S}$,

$$
\begin{aligned}
& P(\ell)=\int_{\mathcal{S}} g(s, \ell)\left(\Upsilon(s) P(s) \Upsilon(s)^{*}+\pi(s) \Psi(s) \Psi(s)^{*}\right) \mu(d s) \\
& S(s)=\Upsilon(s)^{*} \mathcal{E}(S)(s) \Upsilon(s)+\Phi(s)^{*} \Phi(s)
\end{aligned}
$$

Notice that, from Corollary A.2.9 there exists a unique solution $P \in \mathbb{H}_{1}^{2 n+} \cap \mathbb{H}_{\text {sup }}^{2 n+}$ for (7.41) and a unique solution $S \in \mathbb{H}_{\text {sup }}^{2 n+}$ for (7.42). These solutions can be seen respectively as the controllability and observability gramians associated with the stochastically stable MJLS $\mathcal{G}_{c l}$. The next proposition, similar to Theorem 1 by Costa and Tuesta (2004) shows that, as in the MJLS with Markov chain in a finite state space case, the $H_{2}$-norm $\left\|\mathcal{G}_{G}\right\|_{2}$ can be computed from the unique solutions of the $\mathcal{S}$-coupled controllability and observability gramians.

Proposition 7.2.10. Consider the $M J L S \mathcal{G}_{G}$ (7.31) and an admissible control $u \in \mathcal{U}$, such that the closed loop system $\mathcal{G}_{c l}$ (7.38) is stochastically stable. Then it holds that

$$
\left\|\mathcal{G}_{G}\right\|_{2}^{2}=\int_{\mathcal{S}} \int_{\mathcal{S}} \pi(\ell) g(\ell, s) \operatorname{tr}\left(\Psi(\ell)^{*} S(s) \Psi(\ell)\right) \mu(d s) \mu(d \ell)=\int_{\mathcal{S}} \operatorname{tr}\left(\Phi(\ell) P(\ell) \Phi(\ell)^{*}\right) \mu(d \ell) .
$$

Proof. From (7.38) and (7.42) for $\{w(k) ; k \in \mathbb{N}\}$ given by $w=(w(0), w(1), \ldots), w(0)=e_{s}$, $w(k)=0$ for each $k>0$, and $\mathcal{P}\left(\theta_{0} \in B\right)=\int_{B} \pi(\ell) \mu(d \ell)$, for $B \in \mathcal{B}(\mathcal{S})$, it follows that

$$
\begin{aligned}
E\left(z_{s}(k)^{*} z_{s}(k)\right) & =E\left(\mathbf{v}(k)^{*} \Phi(\theta(k))^{*} \Phi(\theta(k)) \mathbf{v}(k)\right) \\
& =E\left(\mathbf{v}(k)^{*}\left(S(\theta(k))-\Upsilon(\theta(k))^{*} \mathcal{E}(S)(\theta(k)) \Upsilon(\theta(k))\right) \mathbf{v}(k)\right) \\
& =E\left(E\left(\mathbf{v}(k)^{*}\left(S(\theta(k))-\Upsilon(\theta(k))^{*} S(\theta(k+1)) \Upsilon(\theta(k))\right) \mathbf{v}(k) \mid \mathcal{F}_{k}\right)\right) \\
& =E\left(\mathbf{v}(k)^{*} S(\theta(k)) \mathbf{v}(k)-\mathbf{v}(k+1)^{*} S(\theta(k+1)) \mathbf{v}(k+1)\right) .
\end{aligned}
$$

From (7.40), (7.43), $\mathbf{v}(1)=\Psi\left(\theta_{0}\right) e_{s}$, and since $\mathcal{G}_{c l}$ (7.38) is stochastically stable, $E\left(\mathbf{v}(k)^{*} \mathbf{v}(k)\right) \rightarrow 0$ as $k \rightarrow \infty$, one gets that

$$
\begin{aligned}
\left\|z_{s}\right\|_{2}^{2} & =\sum_{k=1}^{\infty} E\left(\left\|z_{s}(k)\right\|^{2}\right)=E\left(e_{s}^{*} \Psi\left(\theta_{0}\right)^{*} S(\theta(1)) \Psi\left(\theta_{0}\right) e_{s}\right) \\
& =\int_{\mathcal{S}} \int_{\mathcal{S}} e_{s}^{*} \Psi(\ell)^{*} S(t) \Psi(\ell) e_{s} g(\ell, t) \pi(\ell) \mu(d t) \mu(d \ell) \\
& =\int_{\mathcal{S}} e_{s}^{*} \Psi(\ell)^{*} \int_{\mathcal{S}} S(t) g(\ell, t) \mu(d t) \Psi(\ell) e_{s} \pi(\ell) \mu(d \ell) \\
& =\int_{\mathcal{S}} e_{s}^{*} \Psi(\ell)^{*} \mathcal{E}(S)(\ell) \Psi(\ell) e_{s} \pi(\ell) \mu(d \ell),
\end{aligned}
$$


and thus from (7.39) it follows that

$$
\begin{aligned}
\left\|\mathcal{G}_{G}\right\|_{2}^{2} & =\sum_{s=1}^{r} \int_{\mathcal{S}} e_{s}^{*} \Psi(\ell)^{*} \mathcal{E}(S)(\ell) \Psi(\ell) e_{s} \pi(\ell) \mu(d \ell) \\
& =\int_{\mathcal{S}} \pi(\ell) \sum_{s=1}^{r} e_{s}^{*} \Psi(\ell)^{*} \mathcal{E}(S)(\ell) \Psi(\ell) e_{s} \mu(d \ell) \\
& \left.=\int_{\mathcal{S}} \pi(\ell) \operatorname{tr}\left(\Psi(\ell)^{*} \mathcal{E}(S)(\ell) \Psi(\ell)\right) \mu(d \ell)\right],
\end{aligned}
$$

showing that $\left\|\mathcal{G}_{G}\right\|_{2}^{2}=\int_{\mathcal{S}} \int_{\mathcal{S}} \pi(\ell) g(\ell, s) \operatorname{tr}\left(\Psi(\ell)^{*} S(s) \Psi(\ell)\right) \mu(d s) \mu(d \ell)$. From Fubini's theorem, (7.44) and (7.41)-(7.42) it follows that

$$
\begin{aligned}
\left\|\mathcal{G}_{G}\right\|_{2}^{2}= & \int_{\mathcal{S}} \pi(\ell) \operatorname{tr}\left(\Psi(\ell) \Psi(\ell)^{*} \mathcal{E}(S)(\ell)+P(\ell)\left(\Upsilon(\ell)^{*} \mathcal{E}(S)(\ell) \Upsilon(\ell)+\Phi(\ell)^{*} \Phi(\ell)-S(\ell)\right)\right) \mu(d \ell) \\
= & \int_{\mathcal{S}} \operatorname{tr}\left(\int_{\mathcal{S}} \pi(\ell) \Psi(\ell) \Psi(\ell)^{*} g(\ell, s) S(s) \mu(d s)\right. \\
& \left.+P(\ell)\left(\Phi(\ell)^{*} \Phi(\ell)+\Upsilon(\ell) \int_{\mathcal{S}} g(\ell, s) S(s) \mu(d s) \Upsilon(\ell)^{*}-S(\ell)\right)\right) \mu(d \ell) \\
= & \int_{\mathcal{S}} \operatorname{tr}\left(\int_{\mathcal{S}} g(\ell, s) \pi(\ell) \Psi(\ell) \Psi(\ell)^{*} S(s) \mu(d \ell)\right) \mu(d s)+\operatorname{tr}\left(\Phi(\ell) P(\ell) \Phi(\ell)^{*}\right) \mu(d \ell) \\
& +\int_{\mathcal{S}}\left(\int_{\mathcal{S}} \operatorname{tr}\left(g(\ell, s) \Upsilon(\ell) P(\ell) \Upsilon(\ell)^{*} S(s)\right) \mu(d \ell)\right) \mu(d s)-\int_{\mathcal{S}} \operatorname{tr}(P(s) S(s)) \mu(d s) \\
= & \int_{\mathcal{S}} \operatorname{tr}\left(\left[\int_{\mathcal{S}} g(\ell, s)\left(\Upsilon(\ell) P(\ell) \Upsilon(\ell)^{*}+\pi(\ell) \Psi(\ell) \Psi(\ell)^{*}\right) \mu(d \ell)-P(s)\right] S(s)\right) \mu(d s) \\
& +\int_{\mathcal{S}} \operatorname{tr}\left(\Phi(\ell) P(\ell) \Phi(\ell)^{*}\right) \mu(d \ell) \\
= & \int_{\mathcal{S}} \operatorname{tr}\left(\Phi(\ell) P(\ell) \Phi(\ell)^{*}\right) \mu(d \ell),
\end{aligned}
$$

completing the proof.

\subsubsection{Alternative Definition for the $H_{2}$ Optimal Control Prob- lem}

Consider the closed loop system $\mathcal{G}_{c l}$ (7.38) and admissible controllers $\mathcal{G}_{K}$ as in Definition 7.2.7. In this subsection assume that the input sequence $\{w(k) ; k \in \mathbb{N}\}$ is an input noise sequence acting on the system as follows:

Assumption 7.2.11. The input sequence $\{w(k) ; k \in \mathbb{N}\}$ is a q-dimensional zero mean white noise sequence, (that is, $\{w(k) ; k \in \mathbb{N}\}$ is a sequence of independent identically distributed random vectors with $E(w(k))=0$ and $\left.E\left(w(k) w(k)^{\prime}\right)=I\right)$, and that $\{w(k) ; k \in$ 
$\mathbb{N}\}$, the Markov chain $\{\theta(k) ; k \in \mathbb{N}\}$, and the initial state $x_{0}$ are mutually independent.

Next, an auxiliary result is provided.

Proposition 7.2.12. Consider $P(k, \ell)=E\left(\mathbf{v}(k) \mathbf{v}(k)^{*} g(\theta(k-1), \ell)\right)$ for $k \in \mathbb{N}_{1}, \ell \in \mathcal{S}$, where $\mathbf{v}(k)$ is as in (7.38). Then, for $k \in \mathbb{N}_{1}, \ell \in \mathcal{S}$, it holds that

$$
P(k+1, \ell)=\int_{\mathcal{S}}\left(\Upsilon(s) P(k, s) \Upsilon(s)^{*}+\pi(k, s) \Psi(s) \Psi(s)^{*}\right) g(s, \ell) \mu(d s) .
$$

Proof. Recall that $\left.E(w(k))=0, E\left(w(k) w(k)^{\prime}\right)=I\right)$, and that $\{w(k) ; k \in \mathbb{N}\}$, the Markov chain $\{\theta(k) ; k \in \mathbb{N}\}$, and the initial state $x_{0}$ are mutually independent. The result will be shown by induction on $k$. For $k=1$ it follows that

$$
\begin{aligned}
P(2, \ell)= & E\left(\mathbf{v}(2) \mathbf{v}(2)^{*} g(\theta(k), \ell)\right) \\
= & E\left(E\left(\mathbf{v}(2) \mathbf{v}(2)^{*} g(\theta(1), \ell) \mid \mathcal{F}_{0}\right)\right) \\
= & E\left(\int _ { \mathcal { S } } \left(\Upsilon(s) \mathbf{v}(1) \mathbf{v}(1)^{*} \Upsilon(s)^{*}+\Upsilon(s) \mathbf{v}(1) w(1) \Psi(s)^{*}+\Psi(s) w(1) \mathbf{v}(1)^{*} \Upsilon(s)^{*}\right.\right. \\
& \left.\left.+\Psi(s) w(1) w(1) \Psi^{*}\right) g(s, \ell) g\left(\theta_{0}, s\right) \mu(d s)\right) \\
= & \int_{\mathcal{S}}\left(\Upsilon(s) E\left(\mathbf{v}(1) \mathbf{v}(1)^{*} g\left(\theta_{0}, s\right)\right) \Upsilon(s)^{*}+\Upsilon(s) E\left(\mathbf{v}(1) g\left(\theta_{0}, s\right)\right) E(w(1)) \Psi(s)^{*}\right. \\
& +\Psi(s) E(w(1)) E\left(\mathbf{v}(1)^{*} g\left(\theta_{0}, s\right)\right) \Upsilon(s)^{*} \\
& \left.+\Psi(s) E(w(1) w(1)) E\left(g\left(\theta_{0}, s\right)\right) \Psi^{*}\right) g(s, \ell) \mu(d s) \\
= & \int_{\mathcal{S}}\left(\Upsilon(s) P(1, s) \Upsilon(s)^{*}+\pi(1, s) \Psi(s) \Psi(s)^{*}\right) g(s, \ell) \mu(d s) .
\end{aligned}
$$

Suppose the result holds for $k>1$. Then, noticing that $\mathbf{v}(k)$ and $w(k)$ are independent, from Fubini's theorem and Proposition 6.2.2 one gets that

$$
\begin{aligned}
P(k+1, \ell)= & E\left(\mathbf{v}(k+1) \mathbf{v}(k+1)^{*} g(\theta(k), \ell)\right) \\
= & E\left(E\left(\mathbf{v}(k+1) \mathbf{v}(k+1)^{*} g(\theta(k), \ell) \mid \mathcal{F}_{k-1}\right)\right) \\
= & E\left(\int _ { \mathcal { S } } \left(\Upsilon(s) \mathbf{v}(k) \mathbf{v}(k)^{*} \Upsilon(s)^{*}+\Upsilon(s) \mathbf{v}(k) w(k) \Psi(s)^{*}+\Psi(s) w(k) \mathbf{v}(k)^{*} \Upsilon(s)^{*}\right.\right. \\
& \left.\left.+\Psi(s) w(k) w(k) \Psi^{*}\right) g(s, \ell) g(\theta(k-1), s) \mu(d s)\right) \\
= & \int_{\mathcal{S}}\left(\Upsilon(s) E\left(\mathbf{v}(k) \mathbf{v}(k)^{*} g(\theta(k-1), s)\right) \Upsilon(s)^{*}\right. \\
& +\Upsilon(s) E(\mathbf{v}(k) g(\theta(k-1), s)) E(w(k)) \Psi(s)^{*} \\
& +\Psi(s) E(w(k)) E\left(\mathbf{v}(k)^{*} g(\theta(k-1), s)\right) \Upsilon(s)^{*} \\
& \left.+\Psi(s) E(w(k) w(k)) E(g(\theta(k-1), s)) \Psi^{*}\right) g(s, \ell) \mu(d s)
\end{aligned}
$$




$$
=\int_{\mathcal{S}}\left(\Upsilon(s) P(k, s) \Upsilon(s)^{*}+\pi(k, s) \Psi(s) \Psi(s)^{*}\right) g(s, \ell) \mu(d s)
$$

showing (7.45).

The next proposition gives an alternative interpretation for $\left\|\mathcal{G}_{G}\right\|_{2}^{2}$.

Proposition 7.2.13. Consider the $M J L S \mathcal{G}_{G}$ (7.31) and an admissible control $u \in \mathcal{U}$, such that the closed loop system $\mathcal{G}_{c l}$ (7.38) is stochastically stable. Then

$$
\left\|\mathcal{G}_{G}\right\|_{2}^{2}=\limsup _{k \rightarrow \infty} E\left(\|z(k)\|^{2}\right)
$$

Proof. Set $P(k, \ell)=E\left(\mathbf{v}(k) \mathbf{v}(k)^{*} g(\theta(k-1), \ell)\right)$, for $k \in \mathbb{N}, \ell \in \mathcal{S}$, where $\mathbf{v}(k)$ is as in (7.38). From Proposition 7.2.12 it holds, for $k \in \mathbb{N}_{1}, \ell \in \mathcal{S}$, that

$$
P(k+1, \ell)=\int_{\mathcal{S}}\left(\Upsilon(s) P(k, s) \Upsilon(s)^{*}+\pi(k, s) \Psi(s) \Psi(s)^{*}\right) g(s, \ell) \mu(d s)
$$

Moreover, following the same arguments as in Theorem A.2.8 one gets that $P(k) \rightarrow P$ as $k \rightarrow \infty$, where $P \in \mathbb{H}_{1}^{2 n+} \cap \mathbb{H}_{\text {sup }}^{2 n+}$ is the unique solution for the controllability gramian (7.41). Thus, for $k>1$ it holds that

$$
\begin{aligned}
E\left(\|z(k)\|^{2}\right) & =E\left(\operatorname{tr}\left(z(k) z(k)^{*}\right)\right) \\
& =\operatorname{tr}\left(E\left(\Phi(\theta(k)) \mathbf{v}(k) \mathbf{v}(k)^{*} \Phi(\theta(k))^{*}\right)\right) \\
& =\operatorname{tr}\left(E\left(E\left(\Phi(\theta(k)) \mathbf{v}(k) \mathbf{v}(k)^{*} \Phi(\theta(k))^{*} \mid \mathcal{F}_{k-1}\right)\right)\right) \\
& =\operatorname{tr}\left(E\left(\int_{\mathcal{S}} \Phi(s) \mathbf{v}(k) \mathbf{v}(k)^{*} \Phi(s)^{*} g(\theta(k-1), s) \mu(d s)\right)\right) \\
& =\operatorname{tr}\left(\int_{\mathcal{S}} \Phi(s) E\left(\mathbf{v}(k) \mathbf{v}(k)^{*} g(\theta(k-1), s)\right) \Phi(s)^{*} \mu(d s)\right) \\
& =\int_{\mathcal{S}} \operatorname{tr}\left(\Phi(s) P(k, s) \Phi(s)^{*}\right) \mu(d s),
\end{aligned}
$$

and one gets from Proposition 7.2.10 that

$$
\limsup _{k \rightarrow \infty} E\left(\|z(k)\|^{2}\right)=\int_{\mathcal{S}} \operatorname{tr}\left(\Phi(s) P(s) \Phi(s)^{*}\right) \mu(d s)=\left\|\mathcal{G}_{G}\right\|_{2}^{2}
$$

completing the proof.

From Proposition 7.2.13 it can be stated an alternative definition for the $\mathrm{H}_{2}$ optimal control problem: consider that the input sequence $\{w(k) ; k \in \mathbb{N}\}$ is a noise sequence 
as in Assumption 7.2.11, then the Problem 7.2.9 is equivalent to finding a controller $\mathcal{G}_{K}$, such that the closed loop system $\mathcal{G}_{c l}(7.38)$ is stochastically stable and minimizes $\limsup _{k \rightarrow \infty} E\left(\|z(k)\|^{2}\right)$.

\subsubsection{A Separation Principle}

Recall the control and filtering $\mathcal{S}$-coupled algebraic Riccati equations presented in (5.9) and (6.51), respectively, and its stabilizing solutions stated in Definitions A.1.3 and A.2.3. For convenience, the $\mathcal{S}$-coupled algebraic Riccati equations associated with the system $\mathcal{G}_{G}$ (7.31) and its stabilizing solutions are reproduced below.

Definition 7.2.14. - Control $\mathcal{S}$-coupled algebraic Riccati equations: $X \in \mathbb{H}_{\text {sup }}^{n+}$ is the stabilizing solution for the control $\mathcal{S}$-coupled algebraic Riccati equations if it satisfies, for $\ell \in \mathcal{S}$

$$
X(\ell):=C(\ell)^{*} C(\ell)+A(\ell)^{*} \mathcal{E}(X)(\ell) A(\ell)+A(\ell)^{*} \mathcal{E}(X)(\ell) B(\ell) K(X)(\ell)
$$

and

$$
K(\ell):=-\mathcal{K}(X)(\ell)=-\left(D(\ell)^{*} D(\ell)+B(\ell)^{*} \mathcal{E}(X)(\ell) B(\ell)\right)^{-1} B(\ell)^{*} \mathcal{E}(X)(\ell) A(\ell)
$$

stochastically stabilizes $(A, B)$.

Definition 7.2.15. - Filtering $\mathcal{S}$-coupled algebraic Riccati equations: $Y \in \mathbb{H}_{1}^{n+} \cap$ $\mathbb{H}_{\text {sup }}^{n+}$ is the stabilizing solution for the filtering $\mathcal{S}$-coupled algebraic Riccati equations if it satisfies, for $\ell \in \mathcal{S}$,

$$
Y(\ell):=\int_{\mathcal{S}}\left(A(s) Y(s) A(s)^{*}+G(s) G(s)^{*} \pi(s)+M(Y)(s) L(s) Y(s) A(s)^{*}\right) g(s, \ell) \mu(d s)
$$

and

$$
M(\ell):=\mathcal{M}(Y)(\ell)=-A(\ell) Y(\ell) L(\ell)^{*}\left(H(\ell) H(\ell)^{*} \pi(\ell)+L(\ell) Y(\ell) L(\ell)^{*}\right)^{-1}
$$

stochastically stabilizes $(L, A)$.

Recall that the solution to the infinite-horizon LQ optimal control problem with complete information is based on the control $\mathcal{S}$-coupled algebraic Riccati equations (see Section 5.2) and that the filtering $\mathcal{S}$-coupled algebraic Riccati equations are related to the 
design of a minimum mean square linear Markov jump filter (see Section 6.2). From Proposition 5.1.5 one gets that $K \in \mathbb{H}_{\text {sup }}^{n, m}$ and from Proposition 6.1.14 it holds that $M \in \mathbb{H}_{\text {sup }}^{p, n}$. With these results in mind, and from the solution to the $H_{2}$ optimal control problem, a separation principle for the infinite-horizon case is stated in this section.

On the filtered probability space $\left(\Omega, \mathcal{F},\left\{\mathcal{F}_{k}\right\}_{k \in \mathbb{N}}, \mathcal{P}\right)$ consider the following discretetime MJLS $\mathcal{G}_{v}$, where $y(k)$ and $\theta(k)$ are available at each time $k \in \mathbb{N}$,

$$
\mathcal{G}_{v}=\left\{\begin{aligned}
x(k+1) & =A(\theta(k)) x(k)+B(\theta(k)) u(k)+G(\theta(k)) w(k) \\
y(k) & =L(\theta(k)) x(k)+H(\theta(k)) w(k) \\
v(k) & =R(\theta(k))^{1 / 2}(-F(\theta(k)) x(k)+u(k)),
\end{aligned}\right.
$$

with initial condition $\left(x_{0}, \theta_{0}\right)$ having distribution $\widehat{\mu}$, for some probability measure $\widehat{\mu}$ on $\mathbb{C}^{n} \times \mathcal{S}$, such that $E\left(x_{0}\right):=\zeta, E\left(x_{0} x_{0}^{*}\right):=\mathbb{Q}_{0}$, and $x_{0}, \theta_{0}$ are mutually independent. The initial probability measure on $\mathcal{S}$ for $\theta_{0}$ is denoted $\widehat{\mu}_{\mathcal{S}}$, and it is assumed that $\widehat{\mu}_{\mathcal{S}} \ll \mu$, such that there exists $\nu \in \mathbb{H}_{1}^{1+}$ as in Assumption 3.2.2. Consider Assumptions 7.2.1 to 7.2.11, assume that $F \in \mathbb{H}_{\text {sup }}^{n, m}$ stochastically stabilizes $(A, B)$ and that $R \in \mathbb{H}_{\text {sup }}^{m+}$. Recall that, from Assumption 7.2.11, the input sequence $\{w(k) ; k \in \mathbb{N}\}$ is set as a $q$-dimensional zero mean white noise sequence. Also assume that $(A, G)$ is stochastically stabilizable and $(L, A)$ is stochastically detectable, such that there is a unique solution $Y \in \mathbb{H}_{1}^{n+} \cap \mathbb{H}_{\text {sup }}^{n+}$ for the filtering $\mathcal{S}$-coupled algebraic Riccati equations associated with $\mathcal{G}_{v}$ (7.50) (see Theorem A.2.8). In this section consider Markov jump filters as presented in (7.32).

From (7.50), recalling the Definitions (7.34), (7.35) and (7.37), and defining

$$
\Phi_{f}(\theta(k)):=R(\theta(k))^{1 / 2}[-F(\theta(k)) \quad \widehat{C}(\theta(k))]
$$

one gets that the closed loop system associated with $\mathcal{G}_{v}$ is

$$
\left\{\begin{aligned}
\mathbf{v}(k+1) & =\Upsilon(\theta(k)) \mathbf{v}(k)+\Psi(\theta(k)) w(k) \\
v(k) & =\Phi_{f}(\theta(k)) \mathbf{v}(k)
\end{aligned}\right.
$$

The $H_{2}$-norm of the closed loop system $(7.51)$ is denoted $\left\|\mathcal{G}_{v}\right\|_{2}$. Consider the following optimal filtering problem that is useful when dealing with Problem 7.2.9:

Problem 7.2.16. - Filtering Problem: Find a Markov jump filter, as in $\mathcal{G}_{K}$ (7.32), given by $\widehat{A} \in \mathbb{H}_{\text {sup }}^{n}, \widehat{B} \in \mathbb{H}_{\text {sup }}^{p, n}$, and $\widehat{C} \in \mathbb{H}_{\text {sup }}^{n, m}, k \in \mathbb{N}$, and $\widehat{x}_{0} \in \mathbb{R}^{n}$ deterministic, such 
that the closed loop system (7.51) is stochastically stable and that minimizes the cost

$$
\limsup _{k \rightarrow \infty} E\left(\|v(k)\|^{2}\right)
$$

Consider $M \in \mathbb{H}_{\text {sup }}^{p, n}$ and $Y \in \mathbb{H}_{1}^{n+} \cap \mathbb{H}_{\text {sup }}^{n+}$ as in (7.49) and (7.48), respectively. The next result presents a solution to the filtering problem stated in Problem 7.2.16, which follows similar arguments as in Theorem 6.2.7.

Theorem 7.2.17. An optimal solution for the filtering problem posed in Problem 7.2.16 is

$$
\begin{aligned}
& \widehat{A}^{o p}(\ell)=A(\ell)+M(\ell) L(\ell)+B(\ell) F(\ell), \\
& \widehat{B}^{o p}(\ell)=-M(\ell) \\
& \widehat{C}^{o p}(\ell)=F(\ell)
\end{aligned}
$$

and $\widehat{x}_{0}=\zeta$, where $M(\ell)$ is given by (7.49) and $\ell \in \mathcal{S}$. The optimal cost for the filtering problem is

$$
\min _{\mathcal{G}_{K}}\left\|\mathcal{G}_{v}\right\|_{2}^{2}=\int_{\mathcal{S}} \operatorname{tr}\left(\bar{F}(s) Y(s) \bar{F}(s)^{*}\right) \mu(d s),
$$

where $\bar{F}(\ell)=-R(\ell)^{1 / 2} F(\ell), \ell \in \mathcal{S}$.

Proof. Denote by $\hat{x}^{o p}(k), u^{o p}(k)$ the sequences generated by $(7.32)$ when $(\widehat{A}, \widehat{B}, \widehat{C})$ is given by $\left(\widehat{A}^{o p}, \widehat{B}^{o p}, \widehat{C}^{o p}\right)$ as in $(7.52)$; by $x^{o p}(k)$ the sequence generated by $(7.50)$ when the control sequence is $u^{o p}(k)$; and by $\mathbf{v}^{o p}(k), v^{o p}(k)$ the corresponding sequences generated by (7.51). Defining $e^{o p}(k):=x^{o p}(k)-\hat{x}^{o p}(k)$, for $k \in \mathbb{N}$, it follows from (7.50) and (7.32) that

$$
\begin{aligned}
{\left[\begin{array}{l}
x^{o p}(k+1) \\
e^{o p}(k+1)
\end{array}\right]=} & {\left[\begin{array}{cc}
A(\theta(k))+B(\theta(k)) F(\theta(k)) & -B(\theta(k)) F(\theta(k)) \\
0 & A(\theta(k))+M(\theta(k)) L(\theta(k))
\end{array}\right]\left[\begin{array}{c}
x^{o p}(k) \\
e^{o p}(k)
\end{array}\right] } \\
& +\left[\begin{array}{c}
G(\theta(k)) \\
G(\theta(k))+M(\theta(k) H(\theta(k))
\end{array}\right] w(k),
\end{aligned}
$$

and recalling that $F$ stochastically stabilizes $(A, B)$ and that $M$ stochastically stabilizes $(L, A)$ one gets that the above system is stochastically stable. Thus the closed loop system 
for $\left(\widehat{A}^{o p}, \widehat{B}^{o p}, \widehat{C}^{o p}\right)$, given by

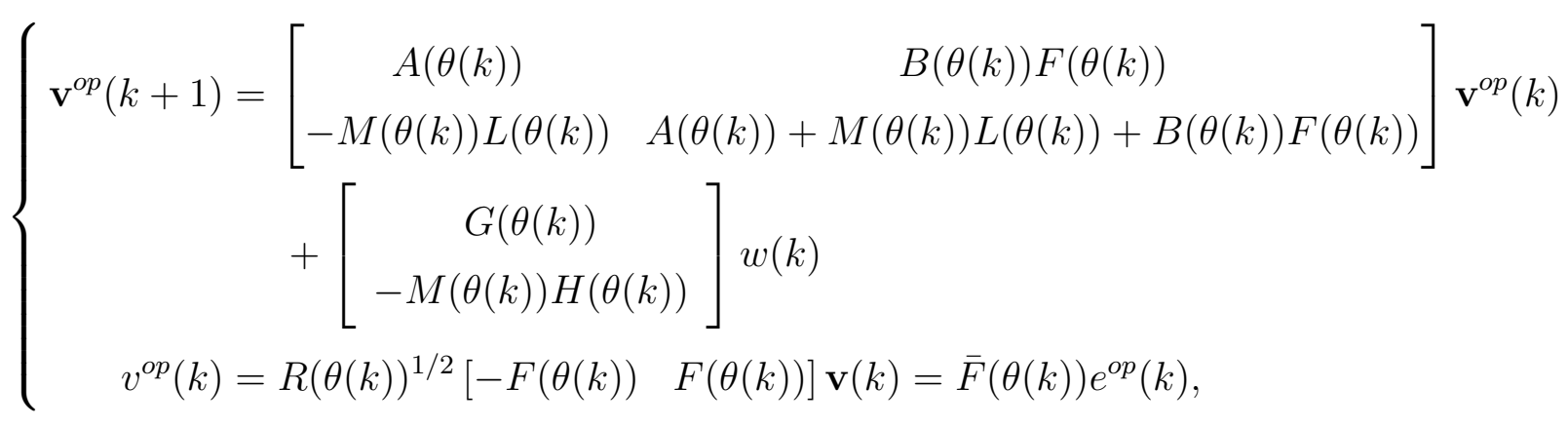

is stochastically stable. Noticing that $v^{o p}(k)=\bar{F}(\theta(k)) e^{o p}(k)$ and following the same arguments as in Theorem 6.2.7 one gets that

$$
\limsup _{k \rightarrow \infty} E\left(\|v(k)\|^{2}\right) \geq \int_{\mathcal{S}} \operatorname{tr}\left(\bar{F}(s) Y(s) \bar{F}(s)^{*}\right) \mu(d s)
$$

and thus from Proposition 7.2.13 it follows that

$$
\min _{\mathcal{G}_{K}}\left\|\mathcal{G}_{v}\right\|_{2}^{2}=\int_{\mathcal{S}} \operatorname{tr}\left(\bar{F}(s) Y(s) \bar{F}(s)^{*}\right) \mu(d s)
$$

completing the proof.

Next, the infinite-horizon $\mathrm{H}_{2}$ optimal control problem, stated in Problem 7.2.9, is addressed. On the filtered probability space $\left(\Omega, \mathcal{F},\left\{\mathcal{F}_{k}\right\}_{\mathrm{T}}, \mathcal{P}\right), \mathrm{T}=\{\ldots,-1,0,1, \ldots\}$, consider again the MJLS $\mathcal{G}_{G}$ given by (7.31) and assume the same hypotheses as in Section 7.2.1. Following the same steps as in (Costa et al., 2005c, Section 6.3), consider a change of variable for the control law in the following form

$$
u(k)=v_{c}(k)+K(\theta(k)) x(k),
$$

where $v_{c}(k)$ is the new control variable. Considering this new variable, and defining $\tilde{A}(\ell):=A(\ell)+B(\ell) K(\ell)$ and $\tilde{C}(\ell):=C(\ell)+D(\ell) K(\ell), \ell \in \mathcal{S}$, the model (7.31) can be rewritten as

$$
\mathcal{G}_{G}=\left\{\begin{aligned}
x(k+1) & =\tilde{A}(\theta(k)) x(k)+B(\theta(k)) v_{c}(k)+G(\theta(k)) w(k) \\
y(k) & =L(\theta(k)) x(k)+H(\theta(k)) w(k) \\
z(k) & =\tilde{C}(\theta(k)) x(k)+D(\theta(k)) v_{c}(k)
\end{aligned}\right.
$$


and the system (7.53) can decomposed such that

$$
\left\{\begin{array}{l}
x(k)=x_{1}(k)+x_{2}(k) \\
z(k)=z_{1}(k)+z_{2}(k)
\end{array}\right.
$$

where

$$
\mathcal{G}_{c}=\left\{\begin{aligned}
x_{1}(k+1) & =\tilde{A}(\theta(k)) x_{1}(k)+G(\theta(k)) w(k) \\
z_{1}(k) & =\tilde{C}(\theta(k)) x_{1}(k)
\end{aligned}\right.
$$

and

$$
\mathcal{G}_{U}=\left\{\begin{aligned}
x_{2}(k+1) & =\tilde{A}(\theta(k)) x_{2}(k)+B(\theta(k)) R(\theta(k))^{-1 / 2} v(k) \\
z_{2}(k) & =\tilde{C}(\theta(k)) x_{2}(k)+D(\theta(k)) R(\theta(k))^{-1 / 2} v(k),
\end{aligned}\right.
$$

with $v(k)=R(\theta(k))^{1 / 2} v_{c}(k), k \in \mathrm{T}$. Notice that the system $\mathcal{G}_{c}(7.55)$ does not depend on the control variable $u(k)$.

Let the operator $\mathcal{G}_{c}(\cdot)$ on $\mathcal{C}^{n}$, associated with system $\mathcal{G}_{c}(7.55)$, be defined as $\mathcal{G}_{c}(w)=$ $\left(\ldots, z(-1), z_{1}(0), z_{1}(1), \ldots\right)$, for $w \in \mathcal{C}^{q}$, and $\mathcal{G}_{c}(w)(k)=z_{1}(k)$. Similarly, define the operators $\mathcal{G}_{G}(\cdot), \mathcal{G}_{U}(\cdot)$ on $\mathcal{C}^{n}$ associated with systems $\mathcal{G}_{G}(7.53)$ and $\mathcal{G}_{U}(7.56)$, respectively. From $(7.54)$ one gets that $z(k)=\mathcal{G}_{c}(w)(k)+\mathcal{G}_{U}(v)(k)$.

Next, it is presented the main result of this section, which can be seen as the separation principle for the infinite-horizon case. It follows similar arguments as in Theorem 6.5 in (Costa et al., 2005c, p. 139).

Theorem 7.2.18. Consider system (7.31) and Markovian jump controllers as in (7.32). Suppose that there exist the stabilizing solutions $X$ and $Y$ for the control and filtering $\mathcal{S}$ coupled algebraic Riccati equations as in (7.46) and (7.48) respectively, and let $K$ and $M$ be as in (7.47) and (7.49) respectively. Then an optimal solution for the $\mathrm{H}_{2}$-control problem is given by $\widehat{A}^{o p}(\ell), \widehat{B}^{o p}(\ell)$, and $\widehat{C}^{o p}(\ell), \ell \in \mathcal{S}$, obtained in Theorem 7.2.17. Moreover, the value of the $\mathrm{H}_{2}$-norm for this control is

$$
\begin{aligned}
\min _{\mathcal{G}_{K}}\|\mathcal{G}\|_{2}^{2}=\int_{\mathcal{S}} & \pi(s) \operatorname{tr}\left(G(s)^{*} \mathcal{E}(X)(s) G(s)\right) \mu(d s) \\
& +\int_{\mathcal{S}} \operatorname{tr}\left(R(s)^{1 / 2} K(s) Y(s) K(s)^{*} R(s)^{1 / 2}\right) \mu(d s),
\end{aligned}
$$

where $R(\ell):=D(\ell)^{*} D(\ell)+B(\ell)^{*} \mathcal{E}(X)(\ell) B(\ell)$, for $\ell \in \mathcal{S}$.

Proof. Consider the closed loop system $\mathcal{G}_{c l}(7.38)$, rewritten below with the output $v(k)=$ 


$$
\begin{aligned}
& R(\theta(k))^{1 / 2} v_{c}(k)=R(\theta(k))^{1 / 2}(u(k)-K(\theta(k)) x(k)), \\
& \mathcal{G}_{v}=\left\{\begin{aligned}
\mathbf{v}(k+1) & =\Upsilon(\theta(k)) \mathbf{v}(k)+\Psi(\theta(k)) w(k) \\
v(k) & =R(\theta(k))^{1 / 2}\left[\begin{array}{ll}
-K(\theta(k)) & \widehat{C}(\theta(k))
\end{array}\right]\left[\begin{array}{c}
x(k) \\
\widehat{x}(k)
\end{array}\right]=\Phi_{f}(\theta(k)) \mathbf{v}(k),
\end{aligned}\right.
\end{aligned}
$$

where $\Upsilon$ and $\Psi$ are as in (7.34) and (7.35) respectively, and

$$
\Phi_{f}(\ell)=R(\ell)^{1 / 2}[-K(\ell) \quad \widehat{C}(\ell)]
$$

$\ell \in \mathcal{S}$. Define the operator $\mathcal{G}_{v}(\cdot)$ on $\mathcal{C}^{n}$, associated with system $\mathcal{G}_{v}(7.57)$, as $\mathcal{G}_{v}(w)=(\ldots$, $v(-1), v(0), v(1), \ldots)$, for $w \in \mathcal{C}^{q}$, and $\mathcal{G}_{v}(w)(k)=v(k)$. Then, from (7.53), (7.55), (7.56) and (7.57), it follows that

$$
z(k)=\mathcal{G}_{G}(w)(k)=\mathcal{G}_{c}(w)(k)+\mathcal{G}_{U}\left(\mathcal{G}_{v}(w)\right)(k) .
$$

It holds that

$$
\begin{aligned}
\left\|\mathcal{G}_{G}(w)\right\|_{2}^{2} & =\left\langle\mathcal{G}_{c}(w)+\mathcal{G}_{U}\left(\mathcal{G}_{v}(w)\right) ; \mathcal{G}_{c}(w)+\mathcal{G}_{U}\left(\mathcal{G}_{v}(w)\right)\right\rangle \\
& =\left\|\mathcal{G}_{c}(w)\right\|_{2}^{2}+\left\langle\mathcal{G}_{U}^{*} \mathcal{G}_{c}(w) ; \mathcal{G}_{v}(w)\right\rangle+\left\langle\mathcal{G}_{v}(w) ; \mathcal{G}_{U}^{*} \mathcal{G}_{c}(w)\right\rangle+\left\langle\mathcal{G}_{U}^{*} \mathcal{G}_{U} \mathcal{G}_{v}(w) ; \mathcal{G}_{v}(w)\right\rangle
\end{aligned}
$$

and from (7.39)-(7.40) that

$$
\left\|\mathcal{G}_{G}\right\|_{2}^{2}=\sum_{s=1}^{q}\left\|\mathcal{G}_{G}\left(w_{s}\right)\right\|_{2}^{2}
$$

where

$$
w_{s}(k)=\left\{\begin{array}{c}
e_{s}, k=0 \\
0, k \neq 0
\end{array}\right.
$$

and $e_{s} \in \mathbb{R}^{q}$ is the unitary vector formed by 1 at the $s^{\text {th }}$ position, 0 elsewhere. From the proof of Theorem 6.5 in (Costa et al., 2005c, p. 139) it follows that

$$
\left\langle\mathcal{G}_{U}^{*} \mathcal{G}_{c}\left(w_{s}\right) ; \mathcal{G}_{v}\left(w_{s}\right)\right\rangle=0
$$

and $\mathcal{G}_{U}^{*} \mathcal{G}_{U}=I$, so that

$$
\left\langle\mathcal{G}_{U}^{*} \mathcal{G}_{U} \mathcal{G}_{v}(w) ; \mathcal{G}_{v}(w)\right\rangle=\left\langle\mathcal{G}_{v}(w) ; \mathcal{G}_{v}(w)\right\rangle=\left\|\mathcal{G}_{v}(w)\right\|_{2}^{2}
$$


From these results one gets that

$$
\left\|\mathcal{G}_{G}\right\|_{2}^{2}=\sum_{s=1}^{q}\left\|\mathcal{G}_{G}\left(w_{s}\right)\right\|_{2}^{2}=\left\|\mathcal{G}_{c}\right\|_{2}^{2}+\left\|\mathcal{G}_{v}\right\|_{2}^{2},
$$

and thus

$$
\min _{\mathcal{G}_{K}}\left\|\mathcal{G}_{G}\right\|_{2}^{2}=\left\|\mathcal{G}_{c}\right\|_{2}^{2}+\min _{\mathcal{G}_{K}}\left\|\mathcal{G}_{v}\right\|_{2}^{2},
$$

since $\mathcal{G}_{c}$ does not depend on the control variable $u$. The solution of $\min _{\mathcal{G}_{K}}\left\|\mathcal{G}_{v}\right\|_{2}^{2}$ is as in Theorem 7.2.17, thus from Proposition 7.2.10 and Theorem 7.2.17 it follows that

$$
\begin{aligned}
\min _{\mathcal{G}_{K}}\|\mathcal{G}\|_{2}^{2}=\int_{\mathcal{S}} & \pi(s) \operatorname{tr}\left(G(s)^{*} \mathcal{E}(X)(s) G(s)\right) \mu(d s) \\
& +\int_{\mathcal{S}} \operatorname{tr}\left(R(s)^{1 / 2} K(s) Y(s) K(s)^{*} R(s)^{1 / 2}\right) \mu(d s),
\end{aligned}
$$

completing the proof.

As for the MJLS case with Markov chain in a finite state space (Costa and Tuesta, 2004; Costa et al., 2005c), it holds from (7.58) that the $H_{2}$-norm can be written as the sum of two $H_{2}$-norms, such that $\left\|\mathcal{G}_{c}\right\|_{2}^{2}$ does not depend on the control $u$, and $\min _{\mathcal{G}_{K}}\left\|\mathcal{G}_{v}\right\|_{2}^{2}$ is obtained from the optimal filter derived in Problem 7.2.16. Notice that $\left\|\mathcal{G}_{c}\right\|_{2}^{2}$ is obtained from the control $\mathcal{S}$-coupled algebraic Riccati equations (7.46), and thus it is associated with an infinite-horizon LQ optimal control problem with complete information, and $\min _{\mathcal{G}_{K}}\left\|\mathcal{G}_{v}\right\|_{2}^{2}$ is obtained based on the filtering $\mathcal{S}$-coupled algebraic Riccati equations (7.48). That is, the optimal controller is obtained from two $\mathcal{S}$-coupled algebraic Riccati equations, one associated with a filtering problem and the other associated with a control problem in which the state variable is fully available. This result is a separation principle for MJLS with Markov chain in a general state space, considering the infinite-horizon case. 


\section{Chapter 8}

\section{Applications}

This chapter is intended to illustrate the application of some of the theoretical results introduced in this thesis. The examples presented in this chapter refer to simplified versions of two realistic control systems found in renewable energy power plants. The dynamics of both systems are subject to abrupt changes due to environmental disturbances. In Section 8.1 it is analyzed a control system of a concentrated solar power plant. This kind of power plant uses mirrors to concentrate sunlight in order to produce the steam that drives a conventional thermoelectric generation system. An application dealing with a wind turbine generator system is discussed in Section 8.2. A wind turbine generator is a device that converts the wind's mechanical energy into electrical power. In both applications the goal is to design an optimal controller for the studied system, which is modeled as a discrete-time MJLS with Markov chain in a general state space such that the system's jump parameter is associated to the atmospheric conditions.

\subsection{LQ Optimal Control for a Solar Thermal Re- ceiver}

The infinite-horizon LQ optimal control for a solar thermal receiver is considered in this section. This example is inspired on the solar thermal receiver model proposed by Sworder and Rogers (1983), and also discussed by Costa et al. (2005c, Chapter 8), adapted for the general state space case. In this example the parameters of the solar thermal receiver were chosen in order to consider some unstable modes of operation. Recall that the infinite-horizon LQ optimal control problem have been addressed in Theorem 5.2 .5 .

Consider a concentrated solar power plant, which is composed of a set of adjustable 


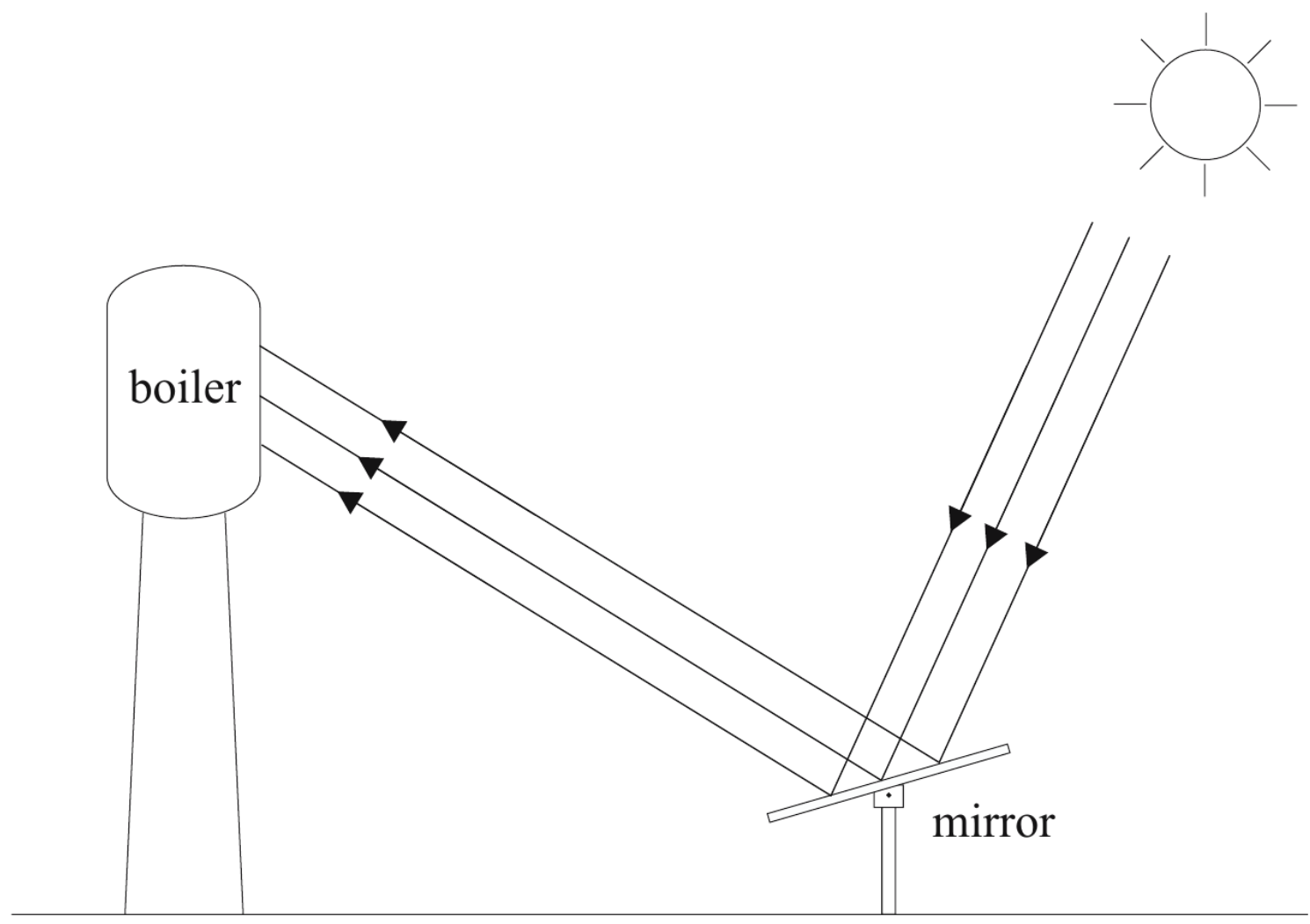

Figure 1: A concentrated solar power plant (Source: Costa et al. (2005c))

mirrors (heliostats), surrounding a tower with a boiler (see Figure 1). The attitude of the heliostats is controlled in order to keep the sunlight focused onto the boiler. The steam produced in the boiler drives a steam turbine power system to generate electricity. One of the control systems in the power plant is responsible for controlling the feedwater flow rate to the boiler in order to maintain the boiler's metal structure temperature within adequate boundaries, thereby controlling the outlet steam temperature. The dynamics of this system is heavily dependent on the instantaneous insolation. In this example it is considered just two atmospheric conditions: 1) sunny (clear sky); and 2) cloudy (dense cloud). The dynamics of the thermal receiver is described, for $k \in \mathbb{N}$, by the following scalar discrete-time MJLS,

$$
x(k+1)=a(\theta(k)) x(k)+b(\theta(k)) u(k),
$$

where $x(k)$ and $u(k)$ are incremental variables associated with the boiler's metal temperature and feed water flow rate, respectively, and $\theta(k)$ is associated with the incident flux into the boiler, modeled in this example by a time-homogeneous Markov chain taking values in the set $\mathcal{S}=\{\{1\} \times[0,1]\} \cup\{\{2\} \times[0,1]\}$. It is assumed that the measurement 
of $x(k)$ and $\theta(k)$ is available through the use of sensors on the plant. For $\theta(k)=(i, t)$, it holds that $\theta(k+1)=(j, U)$ where $U$ is uniformly distributed in the interval [0,1], and $j=1$ with probability $p_{i 1}$ and $j=2$ with probability $p_{i 2}$. Clearly, $p_{i 1}+p_{i 2}=1, p_{i j} \geq 0$, $i, j \in\{1,2\}$. For $(i, t) \in \mathcal{S}$, it holds that

$$
\begin{aligned}
& a(i, t)=a_{i 1}+t\left(a_{i 2}-a_{i 1}\right) \\
& b(i, t)=b_{i 1}+t\left(b_{i 2}-b_{i 1}\right),
\end{aligned}
$$

such that in each atmospheric condition (sunny, represented by $i=1$, or cloudy, represented by $i=2$ ) the dynamics of the system, given by $a(\theta(k))$ and $b(\theta(k))$, can take any value according to a uniform distribution in the continuous interval $\left[a_{i 1}, a_{i 2}\right]$ and $\left[b_{i 1}, b_{i 2}\right]$, respectively. In Table 1 it is presented the parameters considered in this example. It follows that $(1, t)$ corresponds to only stable modes, while $(2, t)$ corresponds to some unstable modes.

Table 1: Parameters for the solar thermal receiver model

\begin{tabular}{cc}
\hline Sunny & Cloudy \\
\hline$a_{11}=0.9$ & $a_{21}=0.95$ \\
$a_{12}=0.7$ & $a_{22}=1.15$ \\
$b_{11}=0.3$ & $b_{21}=0$ \\
$b_{12}=-0.1$ & $b_{22}=0.95$ \\
$p_{11}=0.9767$ & $p_{21}=0.0435$ \\
$p_{12}=0.0233$ & $p_{22}=0.9565$ \\
\hline
\end{tabular}

Consider the infinite-horizon LQ optimal control problem for system (8.1) given by

$$
J\left(x_{0}, \theta_{0}\right)=\inf _{u \in \mathcal{U}} J\left(x_{0}, \theta_{0}, u\right)
$$

where the LQ cost $J\left(x_{0}, \theta_{0}, u\right)$ is given by

$$
J\left(x_{0}, \theta_{0}, u\right)=E\left(\sum_{k=0}^{\infty}\|C(\theta(k)) x(k)\|^{2}+\|D(\theta(k)) u(k)\|^{2}\right)
$$

$C(\ell)=c=0.1884, D(\ell)=d=1$, for $\ell \in \mathcal{S}$, and $u \in \mathcal{U}$ as in Definition 5.2.1.

Consider a state feedback form $\Gamma(\ell), \ell \in \mathcal{S}$, as in Definition 5.2.3 for $(a, b)$. Clearly, 


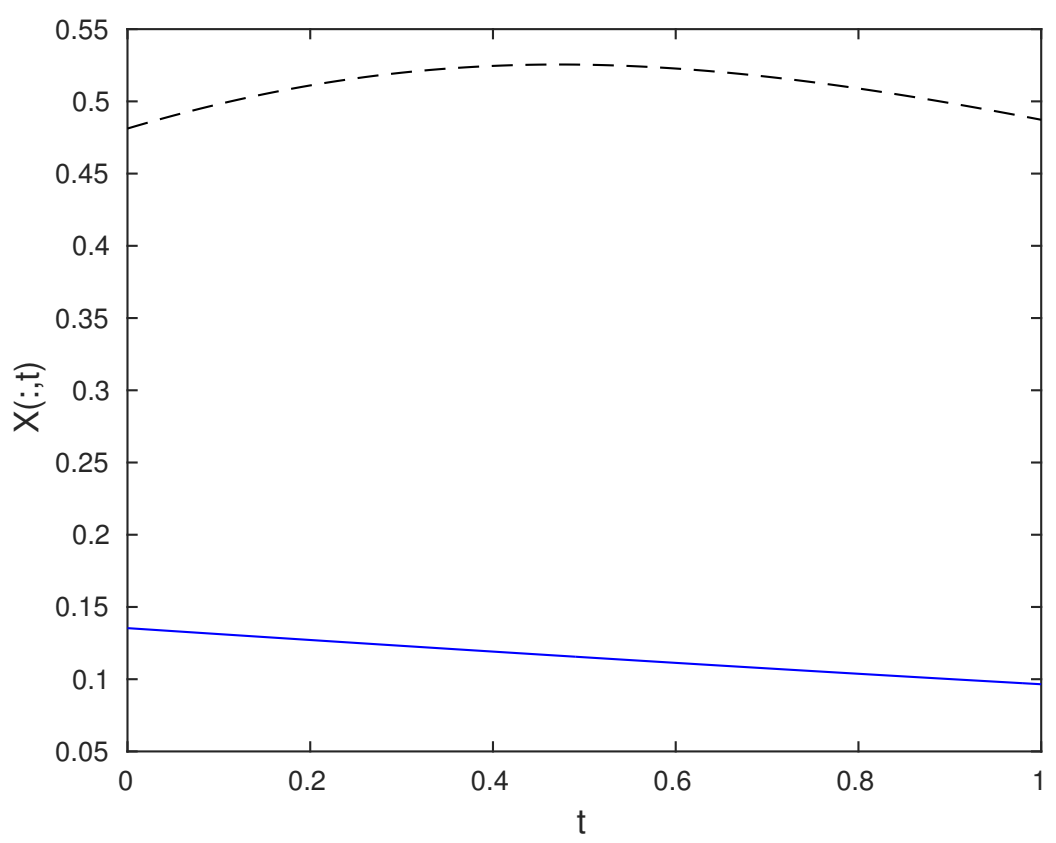

Figure 2: Solution $X$ of the control $\mathcal{S}$-coupled algebraic Riccati equations for the solar thermal receiver

one can find $K \in \mathbb{H}_{\text {sup }}^{1}$ such that $\Gamma(\ell)=a(\ell)+b(\ell) K(\ell)$ satisfies

$$
\|\Gamma(\ell)\|^{2}<\frac{\delta(\ell)}{\int_{\mathcal{S}} \delta(s) g(\ell, s) \mu(d s)}
$$

$\mu$-almost everywhere in $\mathcal{S}$, for some $\{\delta(\ell) ; \ell \in \mathcal{S}\}$ such that $\delta(\ell) \geq 0$, ess-sup $\{\delta(\ell) ; \ell \in$ $\mathcal{S}\}<\infty$. For instance, consider $\delta(i, t)=\gamma_{i} t^{2}$, with $\gamma_{i}>0, i \in\{1,2\}$. Then from Corollary 4.3.5, one gets that the system (8.1) is stochastically stabilizable. Following similar reasoning one gets that the system is also stochastically detectable and thus the Theorem 5.2.5 holds. In this case, one is interested in solutions $X(i, t) \geq 0, i \in\{1,2\}$, $0 \leq t \leq 1$, satisfying the equation

$$
X(i, t)=c^{2}+\frac{(a(i, t) d)^{2} \zeta(i)}{d^{2}+b(i, t)^{2} \zeta(i)},
$$

where

$$
\begin{aligned}
\zeta(i) & =p_{i 1} \int_{0}^{1} X(1, s) d s+p_{i 2} \int_{0}^{1} X(2, s) d s \\
a(i, t) & =a_{i 1}+t\left(a_{i 2}-a_{i 1}\right) \\
b(i, t) & =b_{i 1}+t\left(b_{i 2}-b_{i 1}\right)
\end{aligned}
$$




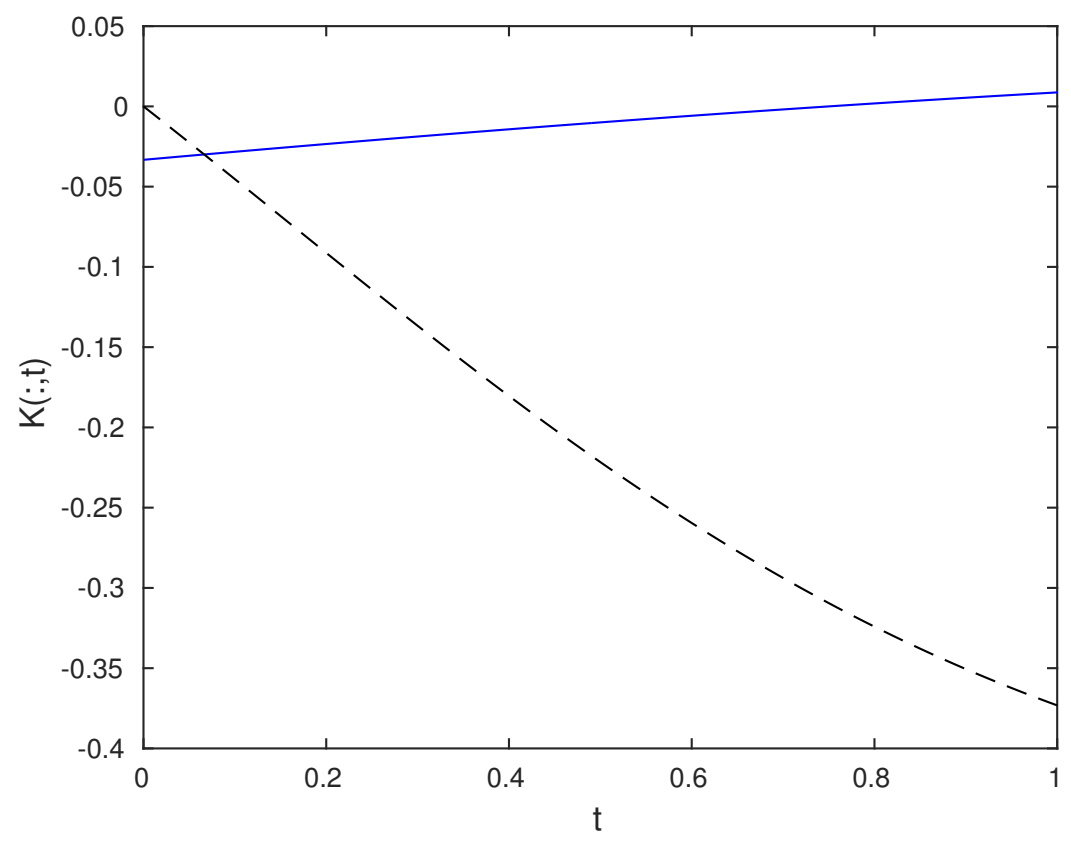

Figure 3: Optimal gains $K$ for the solar thermal receiver

and the optimal control is given by

$$
K(i, t)=-\frac{a(i, t) b(i, t) \zeta(i)}{d^{2}+b(i, t)^{2} \zeta(i)} .
$$

The above equations can be numerically solved by considering a finite grid approximation for the space $\mathcal{S}$. In Figure 2 it is presented the solution $X(1, t)$ (solid line) and $X(2, t)$ (dashed line), and in Figure 3 it is presented the respective optimal controller gains $K(1, t)$ (solid line) and $K(2, t)$ (dashed line), $0 \leq t \leq 1$.

\subsection{LQ Optimal Control with Partial Information for a Wind Turbine Generator System}

In this section the finite-horizon LQ optimal control with partial information for a wind turbine generator system (WTGS) subject to different wind regimes is presented. Refer to Bianchi et al. (2007, Chapter 3) for a detailed discussion on the WTGS modeling. Following similar steps as in (Lin et al., 2015, Section III), initially a MJLS model for the WTGS is presented. The model is obtained from several interconnected subsystems: a rotor, a pitch actuator, a mechanical, and an electrical subsystem (see Figure 4). Next, a finite-horizon LQ optimal control problem with partial information for the WTGS is 
stated. From the result in Theorem 7.1.13 this control problem is addressed and a Monte Carlo simulation of possible trajectories for the system's variables is presented.

The system considered in this example is an onshore three-bladed upwind variablespeed and variable-pitch WTGS. The controller has access to a measurable variable as well as the jump parameter associated to the abrupt wind speed changes. It is assumed that the available control variables are the generator electromagnetic torque and the blade pitch angle. Also, it is assumed that wind speeds are greater than the WTGS rated wind speed, and the controller objective is to keep the captured power within adequate boundaries in order to avoid excessive aerodynamical and mechanical loads.

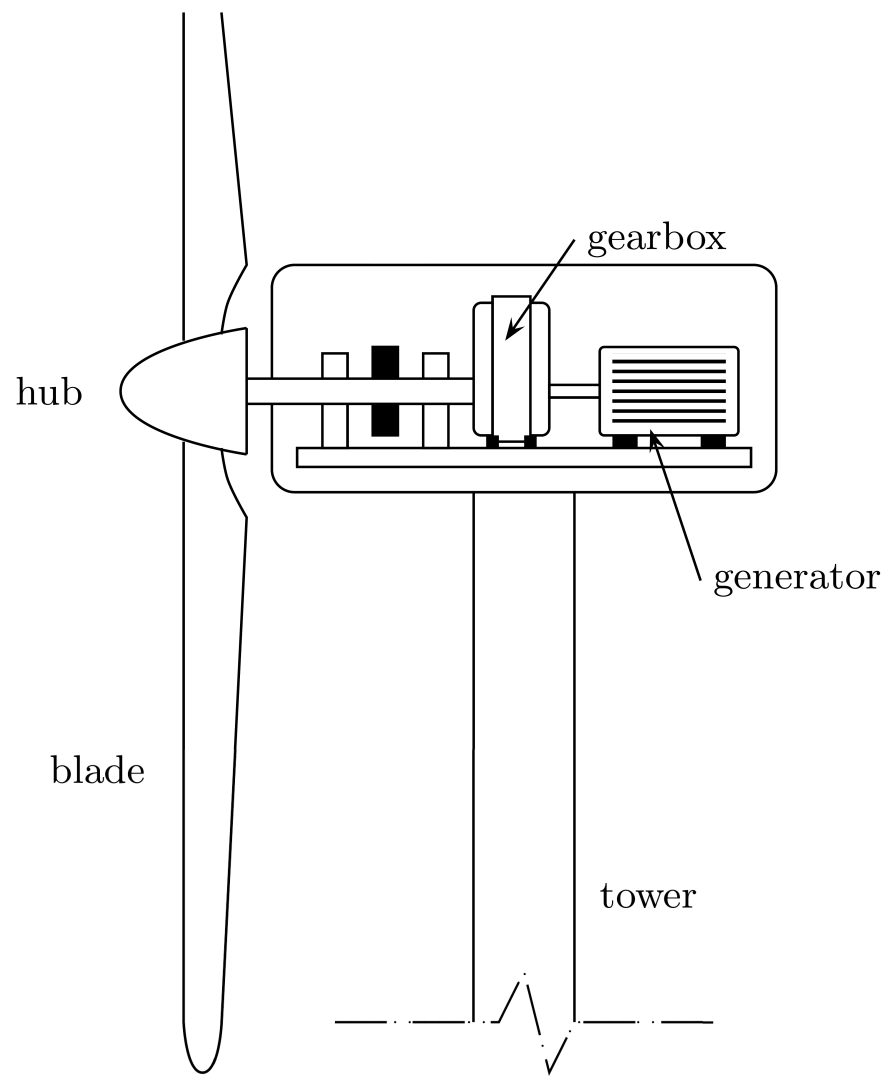

Figure 4: A WTGS with horizontal-axis wind turbine (Source: Bianchi et al. (2007))

Based on Betz' theory, the relation between wind speed and mechanical power extracted from the wind is given by

$$
P_{r}=\frac{\rho \pi R^{2} V^{3} C_{P}(\lambda, \beta)}{2}
$$

and the output mechanical torque of the wind turbine $T_{r}$ can be calculated from the power 
$P_{r}$ by

$$
T_{r}=\frac{P_{r}}{\omega_{r}}
$$

where $\rho$ is the mass density of air, $R$ is the turbine rotor radius, $V$ is the wind speed at hub height, $C_{P}$ is the power coefficient, and $\omega_{r}$ is the hub angular velocity. Note that $\pi R^{2}$ is the swept area by the turbine blades. The tip speed ratio $\lambda$ is defined as the ratio of the linear speed at the tip of blades to the speed of the wind and can be expressed as

$$
\lambda=\frac{\omega_{r} R}{V}
$$

The power coefficient $C_{P}$ can be expressed as a nonlinear function of both the blade tip speed ratio $\lambda$, which is determined by the blade design, and the blade pitch angle $\beta$. It is usually provided by manufacturers or it can be empirically determined for a particular turbine. In this example it is adopted an approximation as follows (Garcia-Sanz and Houpis, 2012, Chapter 12),

$$
C_{P}(\lambda, \beta)=c_{1}\left(\frac{c_{2}}{\lambda_{i}}-c_{3} \beta-c_{4}\right) \exp \left(-\frac{c_{5}}{\lambda_{i}}\right)
$$

where

$$
\lambda_{i}=\left(\frac{1}{\lambda+c_{6} \beta}-\frac{c_{7}}{\beta^{3}+1}\right)^{-1}
$$

and $c_{1}, c_{2}, \ldots, c_{7}$ are parameters that characterize a turbine. The main control objective when the wind speed is greater than the WTGS rated wind speed is to maintain the mechanical power around the rated power of the turbine.

The pitch angle actuator can be modeled as a first-order dynamic system given by

$$
\dot{\beta}=\frac{1}{\tau_{\beta}}\left(\beta_{\text {ref }}-\beta\right)
$$

where $\beta_{\text {ref }}$ is the reference pitch angle, which is a control variable, and $\tau_{\beta}$ is the time constant of the actuator.

In the WTGS proposed in this example, it is considered that the hub is fixed to the rotor shaft which drives the generator through a gearbox. This transmission mechanism dynamics can be modeled as a two mass model given by

$$
\begin{aligned}
& J_{r} \dot{\omega}_{r}=T_{r}-\eta T_{g}, \\
& J_{g} \dot{\omega}_{g}=T_{g}-T_{e},
\end{aligned}
$$




$$
\omega_{g}=\eta \omega_{r},
$$

where $J_{r}$ is the inertia of the turbine rotor (blades, hub and rotor shaft), $\eta$ is the gearbox ratio, $T_{g}$ is the torque of the generator shaft, $J_{g}$ is the inertia of the generator rotor (gearbox and generator shaft), and $T_{e}$ is the electromagnetic torque. For simplicity, in (8.10)-(8.12) the torsional stiffness and damping associated with the wind turbine drivetrain are ignored. Also for simplicity, it is assumed that the electric power produced by the WTGS is given by $P=\omega_{g} T_{e}$.

Set the state variable $x_{\mathrm{NL}}=\left[\begin{array}{ll}\beta & \omega_{r}\end{array}\right]^{\prime}$, the control input variable $u=\left[\begin{array}{ll}\beta_{\mathrm{ref}} & T_{e}\end{array}\right]$, and the measured output variable $y_{\mathrm{NL}}=\omega_{r}+h \xi$, where $h$ is a constant and $\xi$ is a measurement noise. From (8.5)-(8.12) one gets a nonlinear model for the WTGS given by

$$
\begin{aligned}
& \dot{x}_{\mathrm{NL}}=\left[\begin{array}{c}
\dot{\beta} \\
\dot{\omega}_{r}
\end{array}\right]=\left[\begin{array}{l}
\frac{-\beta+\beta_{\mathrm{ref}}}{\tau_{\beta}} \\
\frac{T_{r}-\eta T_{e}}{J_{r}+\eta^{2} J_{g}}
\end{array}\right] \\
& y_{\mathrm{NL}}=\omega_{r}+h \xi,
\end{aligned}
$$

where $T_{r}$ is given by (8.6). For a more complete model for the WTGS, refer to the book by Elkington et al. (2012) or Bianchi et al. (2007).

The wind speed signal $V$ can be expressed in a given time instant as

$$
V=\bar{V}+v
$$

where $\bar{V}$ is the mean wind speed, obtained as the average of the instantaneous speed over a time period, and $v$ denotes the atmospheric turbulence (see Bianchi et al., 2007). It is worth pointing out that accurate wind speed measurements are rarely available in practice, but it is possible to estimate the mean wind speed based on measurements of rotor speed, blade pitch angle and generator torque using a filter, for example, an extended Kalman filter (see Bakka et al., 2014). In this example it is assumed that the wind speed is precisely known.

From (8.5) and (8.6), the linearization of $T_{r}$ around $\bar{V}$ results in the approximation

$$
T_{r} \approx \bar{T}_{r}(\bar{V})+T_{r \mathrm{~L}}(\bar{V}) v
$$


where

$$
\begin{gathered}
\bar{T}_{r}(\bar{V})=\left.T_{r}\right|_{V=\bar{V}}=\left.\frac{\rho \pi R^{2} V^{3} C_{P}(\lambda, \beta)}{2 \omega_{r}}\right|_{V=\bar{V}} \\
T_{r \mathrm{~L}}(\bar{V})=\left.\frac{\partial T_{r}}{\partial V}\right|_{V=\bar{V}}=\frac{1}{2}\left[\frac{3 \rho \pi R^{2} V^{2} C_{P}(\lambda, \beta)}{\omega_{r}}-\rho \pi R^{3} V \frac{\partial C_{P}(\lambda, \beta)}{\partial \lambda}\right]_{V=\bar{V}} .
\end{gathered}
$$

From (8.13) and (8.16) it follows that

$$
\dot{x}_{\mathrm{NL}}=\left[\begin{array}{c}
\overline{-\beta} \\
\frac{\bar{T}_{r}(\bar{V})}{J_{r}+\eta^{2} J_{g}}
\end{array}\right]+\left[\begin{array}{c}
\frac{\beta_{\mathrm{ref}}}{\tau_{\beta}} \\
-\frac{\eta T_{e}}{J_{r}+\eta^{2} J_{g}}
\end{array}\right]+\left[\begin{array}{c}
0 \\
\frac{T_{r \mathrm{~L}}(\bar{V}) v}{J_{r}+\eta^{2} J_{g}}
\end{array}\right] .
$$

The terms in (8.17) are associated with the state variable, control input variable and atmospheric turbulence respectively.

For a given mean wind speed $\bar{V}$ it is possible to linearize (8.17) and (8.14) around the WTGS operation point $\bar{x}=\left[\begin{array}{ll}\bar{\beta} & \bar{\omega}_{r}\end{array}\right]^{\prime}$, such that $x_{\mathrm{NL}}=\bar{x}+x_{\mathrm{L}}$, and get

$$
\begin{aligned}
\dot{x}_{\mathrm{L}} & =\mathcal{A}(\bar{V}) x_{\mathrm{L}}+\mathcal{B} u+\mathcal{G}(\bar{V}) v \\
y_{\mathrm{L}} & =L x_{\mathrm{L}}+h \xi
\end{aligned}
$$

where

$$
\begin{aligned}
& \mathcal{A}(\bar{V})= {\left[\begin{array}{cc}
-\frac{1}{\tau_{\beta}} & 0 \\
\left.\frac{\partial \bar{T}_{r}(\bar{V})}{\partial \beta}\right|_{x_{\mathrm{NL}}=\bar{x}} & \left.\frac{\partial \bar{T}_{r}(\bar{V})}{\partial \omega_{r}}\right|_{x_{\mathrm{NL}}=\bar{x}} \\
J_{r}+\eta^{2} J_{g} & \eta^{2} J_{g}
\end{array}\right], } \\
& \mathcal{B}=\left[\begin{array}{cc}
\frac{1}{\tau_{\beta}} & 0 \\
0 & -\frac{\eta}{J_{r}+\eta^{2} J_{g}}
\end{array}\right], \\
& \mathcal{G}(\bar{V})=\left[\begin{array}{cc}
0 \\
\frac{T_{r \mathrm{~L}}(\bar{V})}{J_{r}+\eta^{2} J_{g}}
\end{array}\right], \\
& L=\left[\begin{array}{ll}
0 & 1
\end{array}\right] .
\end{aligned}
$$

In this example, the operation point is defined such that $\omega_{r}$ is the rated hub angular velocity, and $\beta$ is such that $T_{r}$ and $P_{r}$ assume their rated values.

Wind speed is subject to abrupt environmental changes. As a result of this variability, several linearized models are required to characterize the evolution of the WTGS. For 
simplicity, just two wind conditions are considered, named here as strong breeze and gale. This conditions represent respectively mean wind speeds that are slightly higher than rated speed, and pretty higher than rated speed. Assume that the wind regime can be modeled by a time-homogeneous Markov chain $\{\theta(k) ; k \in \mathbb{N}\}$ with state space given by $\mathcal{S}=\{\{1\} \times[0,1]\} \cup\{\{2\} \times[0,1]\}$. For $\theta(k)=(i, t) \in \mathcal{S}$ the wind condition is represented by $i$, with $i=1$ being strong breeze and $i=2$ being gale, and the mean wind speed $\bar{V}$ depends on $\theta(k)$ as follows,

$$
\bar{V}(\theta(k))=V_{i 1}+t\left(V_{i 2}-V_{i 1}\right)
$$

where $V_{11}, V_{12}, V_{21}$, and $V_{22}$ are constants. For $\theta(k)=(i, t)$, assume that $\theta(k+1)=(j, U)$ with $U$ being uniformly distributed in the interval $[0,1]$, and $j=1$ with probability $p_{i 1}$, and $j=2$ with probability $p_{i 2}$ (of course $p_{i 1}+p_{i 2}=1$, and $p_{i j} \geq 0, i, j \in\{1,2\}$ ).

Discretizing the model (8.18) using the zero-order holder method with sample time $\Delta_{s}$, and considering output measurement noise, one gets a MJLS model for the WTGS that can be written as follows,

$$
\begin{aligned}
x(k+1) & =A(\theta(k)) x(k)+B(\theta(k)) u(k)+G(\theta(k)) w(k) \\
y(k) & =L x(k)+H w(k),
\end{aligned}
$$

where

$$
\begin{aligned}
& A(\theta(k))=e^{\mathcal{A}(\bar{V}(\theta(k))) \Delta_{s}}, \\
& B(\theta(k))=\left(\int_{\tau=0}^{\Delta_{s}} e^{\mathcal{A}(\bar{V}(\theta(k))) \tau} d \tau\right) \mathcal{B}, \\
& G(\theta(k))=\left[\left(\int_{\tau=0}^{\Delta_{s}} e^{\mathcal{A}(\bar{V}(\theta(k))) \tau} d \tau\right) \mathcal{G}(\bar{V}(\theta(k))) \quad 0\right], \\
& H=\left[\begin{array}{ll}
0 & h
\end{array}\right] \text {, } \\
& w(k)=\left[\begin{array}{l}
v \\
\xi
\end{array}\right] \text {. }
\end{aligned}
$$

Assume the same hypothesis as in Section 7.1. It imposes that the sequence $\{w(k) ; k \in \mathbb{N}\}$ is a 2-dimensional zero mean white noise sequence.

Regarding the WTGS, one is interested in solving a control problem as the one stated in Definition 7.1.8, that is, one is interested in finding $u \in \mathcal{U}$ that minimizes the quadratic $\operatorname{cost} J\left(x_{0}, \theta_{0}, u\right)$ given by (7.3). The control objective is to keep the WTGS mechani- 
cal power held constant by controlling the reference blade pitch angle and the generator electromagnetic torque. The quadratic cost represents a compromise between two performance objectives: minimum tracking error and minimum control effort. The equations (7.5) and (7.17) can be numerically solved by considering a finite grid approximation for the space $\mathcal{S}$, and the optimal controller can be derived from Theorem 7.1.13.

Next, a Monte Carlo simulation for the WTGS (8.20) is provided, considering the optimal controller. The parameters for the WTGS considered in this simulation are based on (Jonkman et al., 2009; Zhang et al., 2014) and presented in Table 2. The WTGS rated wind speed is $11.4 \mathrm{~m} / \mathrm{s}$, and it is adopted $V_{11}=12, V_{12}=14, V_{21}=15$ and $V_{22}=17 \mathrm{in}(8.19)$. The input noise sequence $\{w(k) ; k=0,1, \ldots, \tau\}$ is assumed a Gaussian white noise sequence. Parameters in the power coefficient approximation (8.8) have been assigned to $c_{1}=0.39, c_{2}=116, c_{3}=0.4, c_{4}=5, c_{5}=16.5, c_{6}=0.089$, and $c_{7}=0.035$ (Garcia-Sanz and Houpis, 2012, Chapter 12). Regarding the quadratic cost, for simplicity, it was adopted, for all $\ell \in \mathcal{S}, C(\ell)^{*} C(\ell)=S(\ell)=I$ and $D(\ell)^{*} D(\ell)=I$ in (7.3). The sample time interval considered was $\Delta_{s}=6$ seconds, and the simulation lasted for 20 minutes, so that $\tau=200$.

Table 2: Parameters for the wind turbine generator system

\begin{tabular}{ccc}
\hline Parameter & Value & Unit \\
\hline$\omega_{r}$ rated & 1.2671 & $\mathrm{rad} / \mathrm{s}$ \\
$T_{r}$ rated & $3.946 \times 10^{6}$ & $\mathrm{~N} \mathrm{~m}$ \\
$P_{r}$ rated & $5.0 \times 10^{6}$ & $\mathrm{~W}$ \\
$p_{11}$ & 0.8 & - \\
$p_{22}$ & 0.7 & - \\
$\rho$ & 1.25 & $\mathrm{~kg} / \mathrm{m}^{3}$ \\
$R$ & 61.5 & $\mathrm{~m}$ \\
$\tau_{\beta}$ & $50 \times 10^{-3}$ & $\mathrm{~s}$ \\
$J_{r}$ & $3.68 \times 10^{7}$ & $\mathrm{~kg} \mathrm{~m}{ }^{2}$ \\
$J_{g}$ & $5.30 \times 10^{2}$ & $\mathrm{~kg} \mathrm{~m}{ }^{2}$ \\
$\eta$ & 97 & - \\
$\Delta_{s}$ & 6 & $\mathrm{~s}$ \\
$h$ & 0.1 & - \\
\hline
\end{tabular}

A Monte Carlo simulation of the closed loop system is presented in Figure 5. This figure contains 1,000 possible trajectories for initial condition $x_{0}=\left[\begin{array}{ll}0 & 0\end{array}\right]^{\prime}$ and $\theta_{0}=(1,0)$. The thick lines in the figure are the expected value trajectories, and data is presented for 
the state variables related to the blade pitch angle $\beta$ and to the hub angular velocity $\omega_{r}$.
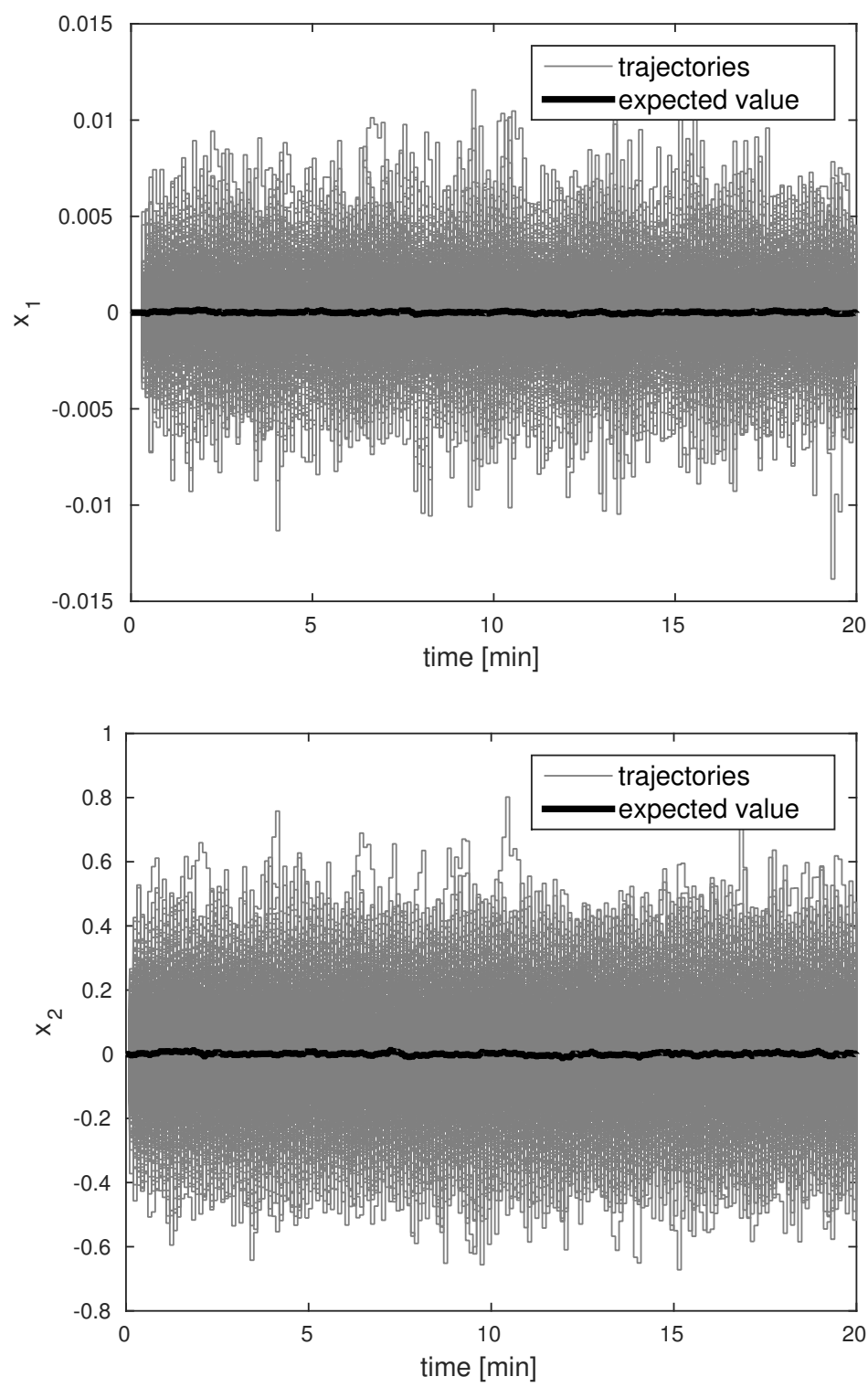

Figure 5: State variables of the wind turbine generator system

The obtained results show that the optimal controller is able to keep the WTGS state variables within adequate boundaries. In this application, several modes of operation have been considered, each one associated to a different wind condition, but only one point of operation was adopted for the WTGS. Recall that the operation point was chosen such that the captured power was limited, thereby avoiding excessive aerodynamical and mechanical loads when wind speed is higher than the WTGS rated wind speed. 


\section{Chapter 9}

\section{Conclusion}

In this thesis several control issues for discrete-time Markov jump linear systems with Markov chain in a Borel space $\mathcal{S}$ have been addressed, including stochastic stability (SS), LQ optimal control synthesis, filter design and a separation principle. This final chapter presents some directions for future research and reviews the main contributions of this thesis.

\subsection{Directions for Future Research}

In the control and filtering problems analyzed in this thesis it was assumed that the current jump parameter $\theta(k)$ was known at each discrete-time instant $k$. Under this hypothesis explicit solutions for the optimal control problems have been obtained, including a separation principle. This is the case in several engineering systems, in which the operating mode of the process can be directly monitored. But in many other practical applications the Markov chain parameter is only partially observed. This section discusses some directions for future research on the optimal control considering partial information of the Markov jump parameter.

In the MJLS literature one can find several works that deal with the partial information of the Markov parameter. One can refer to the papers by Costa et al. (2015), Do Val et al. (2002), Dufour and Elliott (1997), Caines and Zhang (1995), Everdij and Blom (1996), Fragoso $(1988,1990)$, as a sample of papers on this topic.

Do Val et al. (2002) tackled the $H_{2}$ control problem for discrete-time Markov jump linear systems considering the case in which part of, or the total of the Markov states is not accessible to the controller. In this case the non-observed part of the Markov states is grouped into a number of clusters of observations. In particular, the case of a single 
cluster retrieves the situation in which no Markov state is observed (the so-called modeindependent control). Gonçalves et al. (2012) and Fioravanti et al. (2009) also studied the case in which the observations are restricted to clusters of the Markov state. The limit case in which controllers have no information on the jump parameter (mode-independent control) was also tackled by Vargas et al. (2016), Oliveira et al. (2014), Fioravanti et al. (2014), Shu et al. (2010), Costa et al. (1997), Chan and Özgüner (1995), among others.

More recently, Costa et al. (2015) have dealt with the $H_{2}$ control of MJLS with partial information of the jump parameter from a different perspective. They considered that a suitable detector is available and it provides information on the jump parameter by emitting a signal $\widehat{\theta}(k)$. The control design is based just on the information coming from the detector, without recasting the problem as one with complete information as it occurs in the classical literature of optimal stochastic control with partial information. The formulation proposed by Costa et al. (2015) encompasses the cases with perfect information, no information (mode-independent) and cluster case of partial observations of the jump parameter (see Costa et al., 2015, Remark 1), providing a framework that is capable to deal with different scenarios ranging from the case with complete observations to the one with no information on the Markov chain (the mode-independent case).

Costa et al. (2015) have introduced the detector-based approach considering discretetime MJLS with Markov chain taking values in a finite set. Some extension of their method to the general state space case seems an interesting direction for future studies. It is worth pointing out that this research would benefit from some results already obtained for MJLS with Markov chain in a general state space, mainly from the necessary and sufficient conditions for SS presented in Chapter 4 of this thesis.

The partial observation of the jump parameter is also related to the partial information of the transition probability of the Markov chain (see Costa et al., 2015, Section VI), that is, the case that deals with uncertainty in the transition probability matrix. The MJLS with partially unknown transition probabilities has received some attention recently (see, for instance, Zhang et al. (2016), and the references therein). A future research that could pose some control problems considering partially unknown transition probabilities for MJLS with Markov chain in a general state space and tackle them in some way would also be of interest. It is expected that this study would benefit from some of the results already obtained for MJLS with Markov chain in a general state space. 


\subsection{Final Remarks and Summary of Contributions}

This thesis have addressed several control issues for discrete-time MJLS with Markov chain taking values in a general state space.

Necessary and sufficient conditions for SS have been derived in Chapter 4. It was shown that SS is equivalent to the spectral radius of an operator being less than 1 or to the existence of a solution to a "Lyapunov-like" equation.

Based on the SS concept, the LQ optimal control considering complete information was addressed in Chapter 5. The solution to the finite-horizon LQ optimal control problem was derived from the control $\mathcal{S}$-coupled difference Riccati equations and the solution to the infinite-horizon LQ optimal control problem was obtained from the stabilizing positive semi-definite solution of the control $\mathcal{S}$-coupled algebraic Riccati equations. Sufficient conditions for the existence and uniqueness of a stabilizing positive semi-definite solution to the control $\mathcal{S}$-coupled algebraic Riccati equations have been derived in Appendix A.1.

Also based on the concept of SS, the design of minimum mean square linear Markov jump filters was addressed in Chapter 6. A solution to the finite-horizon filtering problem was derived from the associated filtering $\mathcal{S}$-coupled difference Riccati equations. The infinite-horizon filtering problem was also tackled and its solution was obtained in terms of the stabilizing positive semi-definite solution of the filtering $\mathcal{S}$-coupled algebraic Riccati equations. The analysis of the filtering $\mathcal{S}$-coupled algebraic Riccati equations have been presented in Appendix A.2 and sufficient conditions for the existence and uniqueness of a stabilizing positive semi-definite solution to the filtering $\mathcal{S}$-coupled algebraic Riccati equations were obtained.

A separation principle was derived in Chapter 7 based on the results obtained in previous chapters. It was shown that the optimal controller for a partial information optimal control problem separates the optimal control problem into two problems, one associated with a filtering problem and the other one associated with an optimal control problem with complete information. This result is a separation principle for discrete-time MJLS with Markov chain in a general state space.

Examples, based on realistic control systems found in renewable energy power plants, were presented in Chapter 8 in order to illustrate the application of some of the theoretical results introduced in previous chapters.

It is expected that the results obtained in this thesis regarding stochastic stability and optimal control may motivate further research on theoretical issues and practical applications of discrete-time MJLS with Markov chain in a general state space. 


\section{Bibliography}

S. Aberkane, D. Sauter, and J. C. Ponsart. Output feedback robust control of uncertain active fault tolerant control systems via convex analysis. International Journal of Control, 81(2):252-263, 2008.

G. A. Ackerson and K. S. Fu. On the state estimation in switching environments. IEEE Transactions on Automatic Control, 15:10-17, 1970.

F. Auger, M. Hilairet, J. M. Guerrero, E. Monmasson, T. Orlowska-Kowalska, and S. Katsura. Industrial applications of the kalman filter: A review. IEEE Transactions on Industrial Electronics, 60(12):5458-5471, 2013.

T. Bakka, H. R. Karimi, and S. Christiansen. Linear parameter-varying modelling and control of an offshore wind turbine with constrained information. IET Control Theory Applications, 8(1):22-29, 2014.

Y. Bar-Shalom and X. R. Li. Estimation and Tracking. Principles, Techniques and Software. Artech House, 1993.

F. D. Bianchi, H. de Battista, and R. J. Mantz. Wind Turbine Control Systems - Principles, Modelling and Gain Scheduling Design. Springer-Verlag London, 2007.

A. P. Blake and F. Zampolli. Optimal policy in Markov-switching rational expectations models. Journal of Economic Dynamics and Control, 35:1626-1651, 2011.

P. Bolzern, P. Colaneri, and G. De Nicolao. On almost sure stability of discrete-time Markov jump linear systems. In Proc. 43rd IEEE Conference on Decision and Control, volume 3, pages 3204-3208, Bahamas, 2004.

E. K. Boukas. Stochastic Switching Systems: Analysis and Design. Birkhäuser, 2006.

P. E. Caines and J. Zhang. On the adaptive control of jump parameter systems via nonlinear filtering. SIAM Journal on Control and Optimzation, 33:1758-1777, 1995.

F. M. Callier and C. A. Desoer. Linear System Theory. Springer-Verlag, 1991.

H. Chan and Ü. Özgüner. Optimal control of systems over a communication network with queues via a jump system approach. In Proceedings of the 4th IEEE Conference on Control Applications, pages 1148-1153, Albany, New York, 1995.

E. F. Costa and J. B. R. do Val. Weak detectability and the linear-quadratic control problem of discrete-time Markov jump linear systems. International Journal of Control, 75(16-17):1282-1292, 2002. 
E. F. Costa, J. B. R. do Val, and M. D. Fragoso. Weak detectability of LQ problem of discrete-time infinite Markov jump linear systems. Stochastic Analysis and Applications, 23, 2005a.

E. F. Costa, J. B. R. do Val, and M. D. Fragoso. A new approach to detectability of discrete-time infinite Markov jump linear systems. SIAM Journal Control and Optimization, 43:2132-2156, 2005b.

E. F. Costa, A. L. P. Manfrim, and J. B. R. do Val. Weak controllability and weak stabilizability concepts for linear systems with Markov jump parameters. In 2006 American Control Conference, pages 6 pp.--, 2006.

O. L. V. Costa. Discrete-time coupled Riccati equations for systems with Markov switching parameters. Journal of Mathematical Analysis and Applications, 194:197-216, 1995.

O. L. V. Costa and M. V. Araujo. A generalized multi-period mean-variance portfolio optimization with Markov switching parameters. Automatica, 44:2487-2497, 2008.

O. L. V. Costa and D. Z. Figueiredo. Stochastic stability of jump discrete-time linear systems with Markov chain in a general Borel space. IEEE Transactions on Automatic Control, 59:223-227, 2014.

O. L. V. Costa and D. Z. Figueiredo. LQ control of discrete-time jump systems with Markov chain in a general Borel space. IEEE Transactions on Automatic Control, 60 (9):2530-2535, 2015.

O. L. V. Costa and D. Z. Figueiredo. Quadratic control with partial information for discrete-time jump systems with the Markov chain in a general Borel space. Automatica, $66: 73-84,2016$.

O. L. V. Costa and M. D. Fragoso. Stability results for discrete-time linear systems with Markovian jumping parameters. Journal of Mathematical Analysis and Applications, 179:154-178, 1993.

O. L. V. Costa and M. D. Fragoso. Discrete-time LQ-optimal control problems for infinite Markov jump parameter systems. IEEE Transactions on Automatic Control, 40:20762088, 1995.

O. L. V. Costa and M. D. Fragoso. A separation principle for the $H_{2}$-control of continuoustime infinite Markov jump linear systems with partial observations. Journal of Mathematical Analysis and Appications, 331:97-120, 2007.

O. L. V. Costa and S. Guerra. Robust linear filtering for discrete-time hybrid Markov linear systems. International Journal of Control, 75:712-727, 2002a.

O. L. V. Costa and S. Guerra. Stationary filter for linear minimum mean square error estimator of discrete-time Markovian jump systems. IEEE Transactions on Automatic Control, 47:1351-1356, 2002b.

O. L. V. Costa and E. F. Tuesta. Finite horizon quadratic optimal control and a separation principle for Markovian jump linear systems. IEEE Transactions on Automatic Control, 48:1836-1842, 2003. 
O. L. V. Costa and E. F. Tuesta. $H_{2}$-control and the separation principle for discretetime Markovian jump linear systems. Mathematics of Control, Signals and Systems, 16:320-350, 2004.

O. L. V. Costa, J. B. R. do Val, and J. C. Geromel. A convex programming approach to $\mathrm{H}_{2}$-control of discrete-time Markovian jump linear systems. International Journal of Control, 66:557-579, 1997.

O. L. V. Costa, M. D. Fragoso, and R. P. Marques. Discrete-Time Markov Jump Linear Systems. Probability and Its Applications. Springer, 2005c.

O. L. V. Costa, M. D. Fragoso, and M. G. Todorov. Continuous-Time Markov Jump Linear Systems. Springer, 2013.

O. L. V. Costa, M. D. Fragoso, and M. G. Todorov. A detector-based approach for the $\mathrm{H}_{2}$ control of Markov jump linear systems with partial information. IEEE Transactions on Automatic Control, 60:1219-1234, 2015.

A. Czornik. On Control Problems for Jump Linear Systems. Wydawnictwo Politechniki Slaskiej, 2003.

T. Damm. Rational matrix equations in stochastic control, volume 297 of Lecture Notes in Control and Information Sciences. Springer-Verlag, Berlin, 2004.

M. H. A. Davis and R. B. Vinter. Stochastic Modelling and Control. Chapman and Hall, 1985.

D. P. de Farias, J. C. Geromel, J. B. R. do Val, and O. L. V. Costa. Output feedback control of Markov jump linear systems in continuous-time. IEEE Transactions on Automatic Control, 45:944-949, 2000.

J. B. R. Do Val, J. C. Geromel, and A. P. C. Gonçalves. The $H_{2}$-control for jump linear systems: Cluster observations of the Markov state. Automatica, 38:343-349, 2002.

V. Dragan, T. Morozan, and A. M. Stoica. Mathematical Methods in Robust Control of Discrete-Time Linear Stochastic Systems. Springer-Verlag, New York, 2010.

F. Dufour and P. Bertrand. An image based filter for discrete-time Markovian jump linear systems. Automatica, 32:241-247, 1996.

F. Dufour and R. J. Elliott. Adaptive control of linear systems with Markov perturbations. IEEE Transactions on Automatic Control, 43:351-372, 1997.

K. Elkington, J. G. (Han) Slootweg, M. Ghandhari, and W. L. Kling. Wind Power in Power Systems, chapter Reduced-Order Modelling of Wind Turbines, pages 821-847. John Wiley \& Sons Ltd, 2012.

J. S. Evans and R. J. Evans. Image-enhanced multiple model tracking. Automatica, 35: 1769-1786, 1999.

M. H. C Everdij and H. A. P. Blom. Embedding adaptive JLQG into LQ martingale control with a completely observable stochastic control matrix. IEEE Transactions on Automatic Control, 41:424-430, 1996. 
Y. Fang. A new general sufficient condition for almost sure stability of jump linear systems. IEEE Transactions on Automatic Control, 42:378-382, 1997.

Y. Fang and K. A. Loparo. Stochastic stability of jump linear systems. IEEE Transactions on Automatic Control, 47(7):1204-1208, 2002.

Y. Fang, K. A. Loparo, and X. Feng. Almost sure and $\delta$-moment stability of jump linear systems. International Journal of Control, 59:1281-1307, 1994.

X. Feng, K. A. Loparo, Y. Ji, and H. J. Chizeck. Stochastic stability properties of jump linear systems. IEEE Transactions on Automatic Control, 37:38-53, 1992.

A. R. Fioravanti, A. P. C. Goncalves, and J. C. Geromel. Filtering of discrete-time Markov jump linear systems with cluster observation: An approach to Gilbert-Elliot's network channel. pages 2283-2288, 2009.

A. R. Fioravanti, A. P. C. Gonçalves, and J. C. Geromel. Optimal $H_{2}$ and $H_{\infty}$ modeindependent control for generalized Bernoulli jump systems. Journal of Dynamic Systems, Measurement, and Control, 136(1):011004, 2014.

M. D. Fragoso. On a partially observable LQG problem for systems with Markovian jumping parameters. Systems and Control Letters, 10:349-356, 1988.

M. D. Fragoso. A small random perturbation analysis of a partially observable LQG problem with jumping parameter. IMA Journal of Mathematical Control and Information, 7:293-305, 1990.

M. D. Fragoso and O. L. V. Costa. A separation principle for the continuous-time LQproblem with Markovian jump parameters. IEEE Transactions on Automatic Control, 55:2692-2707, 2010.

M. Garcia-Sanz and C. H. Houpis. Wind Energy Systems - Control Engineering Design. CRC Press, 2012.

A. P. C. Gonçalves, A. R. Fioravanti, and J. C. Geromel. Markov jump linear systems and filtering through network transmitted measurements. Signal Processing, 90(10): 2842-2850, 2010.

A. P.C. Gonçalves, A. R. Fioravanti, and J. C. Geromel. $H_{\infty}$ robust and networked control of discrete-time MJLS through LMIs. Journal of the Franklin Institute, 349(6): 2171-2181, 2012.

W. S. Gray, O. R. González, and M. Doğan. Stability analysis of digital linear flight controllers subject to electromagnetic disturbances. IEEE Transactions on Aerospace and Electronic Systems, 36:1204-1218, 2000.

O. Hernández-Lerma and J. B. Lasserre. Discrete-Time Markov Control Processes: Basic Optimality Criteria, volume 30 of Applications of Mathematics. Springer-Verlag, New York, 1996.

O. Hernández-Lerma and J. B. Lasserre. Further topics on discrete-time Markov control processes, volume 42 of Applications of Mathematics. Springer-Verlag, New York, 1999. 
O. Hernández-Lerma and J. B. Lasserre. Markov Chains and Invariant Probabilities, volume 211 of Progress in Mathematics. Birkhauser Basel, Basel, 2003.

O. Hernandez-Lerma and J. B. Lasserre. Discrete-Time Markov Control Processes: Basic Optimality Criteria. Springer New York, 2012.

S. Hu and W.-Y. Yan. Stability robustness of networked control systems with respect to packet loss. Automatica, 43:1243-1248, 2007.

Y. Ji, H. J. Chizeck, X. Feng, and K. A. Loparo. Stability and control of discrete-time jump linear systems. Control Theory and Advanced Technology, 7:247-270, 1991.

J. Jonkman, S. Butterfield, W. Musial, and G. Scott. Definition of a 5-MW reference wind turbine for offshore system development. Technical report, National Renewable Energy Laboratory, 2009.

I. Kordonis and G. P. Papavassilopoulos. On stability and LQ control of MJLS with a Markov chain with general state space. IEEE Transactions on Automatic Control, 59: 535-540, 2014.

M. A. Krasnoselskij, J. A. Lifshits, and A. V. Sobolev. Positive Linear Systems - The Method of Positive Operators, volume 5 of Sigma Series in Applied Mathematics. Heldermann Verlag, Berlin, 1989.

E. Kreyszig. Introductory Functional Analysis with applications. John Wiley and Sons, 1978.

C. S. Kubrusly. Mean square stability for discrete bounded linear systems in Hilbert spaces. SIAM Journal on Control and Optimization, 23:19-29, 1985.

C. S. Kubrusly. On discrete stochastic bilinear systems stability. Journal of Mathematical Analysis and Applications, 113:36-58, 1986.

C. Li, M. Z. Q. Chen, J. Lam, and X. Mao. On exponential almost sure stability of random jump systems. IEEE Transactions on Automatic Control, 57(12):3064 -3077, 2012 .

Z. Lin, J. Liu, and Y. Niu. Regional pole placement of a markovian jump model for wind turbine generator system. In 2015 54th IEEE Conference on Decision and Control $(C D C)$, pages 5055-5060, 2015.

Q. Ling and H. Deng. A new proof to the necessity of a second moment stability condition of discrete-time Markov jump linear systems with real states. Journal of Applied Mathematics, 2012:1-10, 2012.

M. Mahmoud, J. Jiang, and Y. Zhang. Active Fault Tolerant Control Systems: Stochastic Analysis and Synthesis. Springer, New York, 2003.

M. Mariton. Detection delays, false alarm rates and the reconfiguration of control systems. International Journal of Control, 42:449-465, 1989.

M. Mariton. Jump Linear Systems in Automatic Control. Marcel Decker, 1990. 
M. Mariton and P. Bertrand. Reliable flight control systems: Components placement and feedback synthesis. In Proceedings of the 10th IFAC World Congress, pages 150-154, Munich, 1987.

S. Meyn and R. L. Tweedie. Markov Chains and Stochastic Stability. Cambridge University Press, New York, NY, USA, 2nd edition, 2009.

A. W. Naylor and G. R. Sell. Linear Operator Theory in Engineering and Science. Springer-Verlag, second edition, 1982.

R. C. L. F. Oliveira, A. N. Vargas, J. B. R. do Val, and P. L. D. Peres. Mode-independent $\mathrm{H}_{2}$-control of a dc motor modeled as a Markov jump linear system. IEEE Transactions on Control Systems Technology, 22(5):1915-1919, 2014.

P. Seiler and R. Sengupta. An $H_{\infty}$ approach to networked control. IEEE Transactions on Automatic Control, 50:356-364, 2005.

H. H. Shaefer. Topological Vector Spaces. Springer Verlag, Berlin, Heidelberg, New York, 1971.

Z. Shu, J. Lam, and J. Xiong. Static output-feedback stabilization of discrete-time markovian jump linear systems: A system augmentation approach. Automatica, 46(4):687 694, 2010.

A. A. G. Siqueira and M. H. Terra. A fault-tolerant manipulator robot based on $\mathcal{H}_{2}, \mathcal{H}_{\infty}$, and mixed $\mathcal{H}_{2} / \mathcal{H}_{\infty}$ Markovian controls. IEEE-ASME Transactions on Mechatronics, 14:257-263, 2009.

R. Srichander and B. K. Walker. Stochastic stability analysis for continuous-time fault tolerant control systems. International Journal of Control, 57:433-452, 1993.

E. M. Stein and R. Shakarchi. Functional Analysis: Introduction to Further Topics in Analysis. Princeton University Press, 2011.

D. D. Sworder and R. G. Hutchins. Image-enhanced tracking. IEEE Transactions on Aerospace and Eletronic Systems, 25:701-709, 1989.

D. D. Sworder and R. O. Rogers. An LQG solution to a control problem with solar thermal receiver. IEEE Transactions on Automatic Control, 28:971-978, 1983.

D. D. Sworder, P. F. Singer, R. G. Doria, and R. G. Hutchins. Image-enhanced estimation methods. Proc. IEEE, 81:797-812, 1993.

J. K. Tugnait. Detection and estimation for abruptly changing systems. Automatica, 18: 607-615, 1982.

V. M. Ungureanu. Optimal control for linear discrete-time systems with Markov perturbations in Hilbert spaces. IMA Journal of Mathematical Control and Information, 26: 105-127, 2009.

V. M. Ungureanu, V. Dragan, and T. Morozan. Global solutions of a class of discrete-time backward nonlinear equations on ordered Banach spaces with applications to Riccati equations of stochastic control. Optimal Control App. and Methods, 34:164-190, 2013. 
A. N. Vargas, L. Acho, G. Pujol, E. F. Costa, J. Y. Ishihara, and J. B. R. do Val. Output feedback of Markov jump linear systems with no mode observation: An automotive throttle application. International Journal of Robust and Nonlinear Control, 26(9): 1980-1993, 2016.

J. Weidmann. Linear Operators in Hilbert Spaces. Springer-Verlag, 1980.

W. M. Wonham. On a matrix Riccati equation of stochastic control. SIAM Journal of Control, 6:681-697, 1968.

F. Zampolli. Optimal monetary policy in a regime-switching economy: the response to abrupt shifts in exchange rate dynamics. Journal of Economic Dynamics and Control, 30:1527-1567, 2006.

L. Zhang, T. Yang, P. Shi, and Y. Zhu. Analysis and design of Markov jump systems with complex transition probabilities. Springer, 2016.

Z. Zhang, S. R. K. Nielsen, F. Blaabjerg, and D. Zhou. Dynamics and control of lateral tower vibrations in offshore wind turbines by means of active generator torque. Energies, $7(11): 7746,2014$. 


\section{Appendix A}

\section{$\mathcal{S}$-Coupled Algebraic Riccati Equations}

This appendix deals with the control and filtering $\mathcal{S}$-coupled Riccati equations, which are used throughout this thesis. The main issues addressed in this appendix are the conditions for the existence of solutions for the $\mathcal{S}$-coupled algebraic Riccati equations and the asymptotic convergence of the $\mathcal{S}$-coupled Riccati difference equations.

\section{A.1 Control $\mathcal{S}$-Coupled Algebraic Riccati Equations}

This appendix is devoted to present the conditions for the existence and uniqueness of stabilizing positive semi-definite solutions for the control $\mathcal{S}$-coupled algebraic Riccati equations. Section A.1.1 introduces the control $\mathcal{S}$-coupled algebraic Riccati equations, and Section A.1.2 presents some auxiliary results. In Section A.1.3 the conditions for the existence and uniqueness of a stabilizing positive semi-definite solution for the control $\mathcal{S}$-coupled algebraic Riccati equations are derived. The results obtained in this appendix are applied in the design of an optimal controller to address the infinite-horizon optimal control problems in Sections 5.2 and 7.2.

\section{A.1.1 Control $\mathcal{S}$-Coupled Algebraic Riccati Equations}

Recall that the definitions of operators $\mathcal{Q}, \mathcal{K}, \mathcal{R}$ stated in Definition 5.1.4 are such that, for $Z \in \mathbb{H}_{\text {sup }}^{n+}, \ell \in \mathcal{S}$,

$$
\begin{aligned}
& \mathcal{Q}(Z)(\ell):= D(\ell)^{*} D(\ell)+B(\ell)^{*} \mathcal{E}(Z)(\ell) B(\ell), \\
& \mathcal{K}(Z)(\ell):=\mathcal{Q}(Z)(\ell)^{-1} B(\ell)^{*} \mathcal{E}(Z)(\ell) A(\ell), \\
& \mathcal{R}(Z)(\ell):=C(\ell)^{*} C(\ell)+A(\ell)^{*} \mathcal{E}(Z)(\ell) A(\ell) \\
& \quad-A(\ell)^{*} \mathcal{E}(Z)(\ell) B(\ell) \mathcal{Q}(Z)(\ell)^{-1} B(\ell)^{*} \mathcal{E}(Z)(\ell) A(\ell) .
\end{aligned}
$$

Consider a positive $\tau \in \mathbb{N}$. For $k=\tau-1, \ldots, 0$, the control $\mathcal{S}$-coupled Riccati difference equations are defined as follows,

$$
\begin{aligned}
& X^{\tau}(k)=\mathcal{R}\left(X^{\tau}(k+1)\right) \\
& X^{\tau}(\tau)=S
\end{aligned}
$$


where $S \in \widetilde{\mathbb{H}}_{\text {sup }}^{n+}$. Notice that from Proposition 5.1.5 it follows that $X(k) \in \mathbb{H}_{\text {sup }}^{n+}$. Define also the feedback gains $K(k) \in \mathbb{H}_{\text {sup }}^{n, m}$ as

$$
K^{\tau}(k)=-\mathcal{K}\left(X^{\tau}(k+1)\right),
$$

and

$$
V^{\tau}(k)=\mathcal{Q}\left(X^{\tau}(k+1)\right) .
$$

The control $\mathcal{S}$-coupled algebraic Riccati equations are defined as,

$$
X(\ell)=\mathcal{R}(X)(\ell) \quad \mu \text {-almost everywhere in } \mathcal{S} \text {. }
$$

It is assumed that $A \in \widetilde{\mathbb{H}}_{\text {sup }}^{n}, B \in \widetilde{\mathbb{H}}_{\text {sup }}^{m, n}, C \in \widetilde{\mathbb{H}}_{\text {sup }}^{n, r}, D \in \widetilde{\mathbb{H}}_{\text {sup }}^{m, p}$ and $D(\ell)^{*} D(\ell) \geq \epsilon_{D} I$ $\mu$-almost everywhere in $\mathcal{S}$ for some $0<\epsilon_{D} \leq 1$.

\section{A.1.2 Auxiliary Results}

In this subsection, Propositions A.1.1 and A.1.2 are presented. These propositions are useful to obtain the main results that are stated in the next subsection.

Proposition A.1.1. For any initial distribution $\widehat{\mu}$ satisfying Assumption 3.2.2 and $E\left(\left\|x_{0}\right\|^{2}\right)<\infty$, suppose that $\widetilde{X}, X \in \widetilde{\mathbb{H}}_{\text {sup }}^{n+}$ are such that

$$
E\left(x_{0}^{*} X\left(\theta_{0}\right) x_{0}\right) \leq E\left(x_{0}^{*} \widetilde{X}\left(\theta_{0}\right) x_{0}\right) .
$$

Then $X(\ell) \leq \tilde{X}(\ell) \mu$-almost everywhere in $\mathcal{S}$. In particular, if

$$
E\left(x_{0}^{*} X\left(\theta_{0}\right) x_{0}\right)=E\left(x_{0}^{*} \tilde{X}\left(\theta_{0}\right) x_{0}\right)
$$

then $X(\ell)=\widetilde{X}(\ell) \mu$-almost everywhere in $\mathcal{S}$.

Proof. Define the compact set $\Psi=\left\{x \in \mathbb{R}^{n} ;\|x\| \leq 1\right\} \subset \mathbb{R}^{n}$ and the measurable function $v(\cdot, \cdot): \mathbb{R}^{n} \times \mathcal{S} \rightarrow \mathbb{R}$ as $v(x, \ell)=x^{*}(X(\ell)-\tilde{X}(\ell)) x$. Then, for $\ell \in \mathcal{S}$ fixed, $v(\cdot, \ell)$ is a continuous function in $\mathbb{R}^{n}$. Set the function from $\mathcal{S}$ to $\mathbb{R}$ as $\widehat{v}_{1}(\ell)=\max _{\{x \in \Psi\}} v(x, \ell)$. Clearly $\widehat{v}_{1}(\ell) \geq 0$ since $\max _{\{x \in \Psi\}} v(x, \ell) \geq v(0, \ell)=0$. Ordering the $n$ eigenvalues (not necessarily distinct) of $X(\ell)-\widetilde{X}(\ell)$ as $\lambda_{1}(\ell) \geq \ldots \geq \lambda_{n}(\ell)$ it follows that $\widehat{v}_{1}(\ell)=\max \left\{\lambda_{1}(\ell), 0\right\} \geq 0$ (see, for instance, Fact 72 in Callier and Desoer (1991), p. 460), and therefore $X(\ell) \leq$ $\widetilde{X}(\ell)$ if and only if $\widehat{v}_{1}(\ell)=0$. From Proposition D.5 in Hernández-Lerma and Lasserre (1996) there exists a measurable selector $\widehat{f}_{1}(\cdot): \mathcal{S} \rightarrow \Psi$ such that $\widehat{v}_{1}(\ell)=v\left(\widehat{f}_{1}(\ell), \ell\right)$. Consider the measurable set $\Lambda_{1}=\left\{\ell \in \mathcal{S} ; \widehat{v}_{1}(\ell)>0\right\}$. Suppose for the moment that $\mu\left(\Lambda_{1}\right)>0$. As in Proposition 4.2.5 one can find a density $\nu_{1}$ with respect to $\mu$ such that $\nu_{1}(\ell)>0$ for $\ell \in \Lambda_{1}, \nu_{1}(\ell)=0$ for $\ell \in \Lambda_{1}^{c}$, and that $\int_{\Lambda_{1}} \nu_{1}(\ell) \mu(d \ell)=1$. Consider an initial condition $\left(x_{0}, \theta_{0}\right)$ having distribution $\widehat{\mu}_{1}$ defined as follows: take the stochastic kernel $\mathcal{K}_{1}(\cdot, \cdot) \in \mathbb{P}\left(\mathcal{S}, \mathbb{R}^{n}\right)$ as $\mathcal{K}_{1}(\ell, C)=\delta_{\widehat{f}_{1}(\ell)}(C)$ for all $C \in \mathcal{B}\left(\mathbb{R}^{n}\right)$ (where $\delta$. represents the Dirac measure), and set $\widehat{\mu}_{1}(C \times B)=\int_{B} \mathcal{K}_{1}(\ell, C) \nu_{1}(\ell) \mu(d \ell)$, for $C \in \mathcal{B}\left(\mathbb{R}^{n}\right)$ and $B \in \mathcal{B}(S)$, so that Assumption 3.2.2 and $E\left(\left\|x_{0}\right\|^{2}\right)<\infty$ are satisfied. Thus,

$$
E\left(x_{0}^{*}\left(X\left(\theta_{0}\right)-\tilde{X}\left(\theta_{0}\right)\right) x_{0}\right)=E\left(v\left(x_{0}, \theta_{0}\right)\right)
$$




$$
\begin{aligned}
& =\int_{\Lambda_{1}} v\left(\widehat{f}_{1}(\ell), \ell\right) \nu_{1}(\ell) \mu(d \ell) \\
& =\int_{\Lambda_{1}} \widehat{v}_{1}(\ell) \nu_{1}(\ell) \mu(d \ell) \\
& >0
\end{aligned}
$$

since $\widehat{v}_{1}(\ell) \nu_{1}(\ell)>0$ for $\ell \in \Lambda_{1}$. But this contradicts $E\left(x_{0}^{*} X\left(\theta_{0}\right) x_{0}\right) \leq E\left(x_{0}^{*} \widetilde{X}\left(\theta_{0}\right) x_{0}\right)$. Therefore $\mu\left(\Lambda_{1}\right)=0$ must hold.

Proposition A.1.2. Consider a sequence $\left\{X_{n}\right\} \in \widetilde{\mathbb{H}}_{\text {sup }}^{n+}$ with $\sup _{n}\left\|X_{n}\right\|_{\text {sup }} \leq b$, for some $b \geq 0$. Then there are $X_{i f}, X_{s p} \in \widetilde{\mathbb{H}}_{\text {sup }}^{n+}$ such that $\mu$-almost everywhere in $\mathcal{S}$, for every $x \in \mathbb{R}^{n}$,

$$
\liminf _{n \rightarrow \infty} x^{*} X_{n}(\ell) x=x^{*} X_{i f}(\ell) x
$$

and

$$
\limsup _{n \rightarrow \infty} x^{*} X_{n}(\ell) x=x^{*} X_{s p}(\ell) x .
$$

Proof. From the hypothesis that $\sup \left\|X_{n}\right\|_{\text {sup }} \leq b$ one can find a measurable set $\Lambda \subset \mathcal{S}$ such that $\mu\left(\Lambda^{c}\right)=0$ and that $\sup _{n}\left\|X_{n}(\ell)\right\| \leq b$ for all $\ell \in \Lambda$. For each $\ell \in \Lambda$ set $\alpha(x, \ell)=\liminf _{n \rightarrow \infty} x^{*} X_{n}(\ell) x$. Let $e_{i}$ be the unit vector in the $i^{\text {th }}$ coordinate direction in $\mathbb{R}^{n}$. Following similar steps as in the proof of Proposition D.1.4 by Davis and Vinter (1985) one can define for each $\ell \in \Lambda$ the elements $(i, j)$ of the symmetric matrix $X_{i f}(\ell)$ as

$$
\begin{aligned}
\left(X_{i f}(\ell)\right)_{i i} & =\alpha\left(e_{i}, \ell\right), \quad \text { and } \\
\left(X_{i f}(\ell)\right)_{i j} & =\frac{1}{2}\left(\alpha\left(e_{i}+e_{j}, \ell\right)-\alpha\left(e_{i}, \ell\right)-\alpha\left(e_{i}, \ell\right)\right),
\end{aligned}
$$

for $i \neq j$ (for $\ell \in \Lambda^{c}$ one can define $X_{i f}(\ell)$ in an arbitrary way). Then the elements $\left(X_{i f}(\cdot)\right)_{i j}$ are measurable functions from $\mathcal{S}$ to $\mathbb{R}$ and, as shown in Proposition D.1.4 by Davis and Vinter (1985), $\liminf _{n \rightarrow \infty} x^{*} X_{n}(\ell) x=x^{*} X_{i f}(\ell) x$. Similar reasoning shows the second result, completing the proof.

\section{A.1.3 Stationary Case}

The solution to the infinite-horizon optimal control problem, stated in Problem 5.2.2, is related to the existence of solutions $X \in \widetilde{\mathbb{H}}_{\text {sup }}^{n+}$ to the control $\mathcal{S}$-coupled algebraic Riccati equations (A.4). Furthermore, the interest is focused in a stabilizing solution, as defined in the sequel.

Definition A.1.3. $X$ is a stabilizing solution for (A.4) if $X \in \widetilde{\mathbb{H}}_{\text {sup }}^{n+}$, and $K=-\mathcal{K}(X)$ stochastically stabilizes $(A, B)$ in the sense of Definition 5.2.3.

The next result presents a sufficient condition, based on the concept of stochastic stabilizability (see again Definition 5.2.3), for the existence of a solution for (A.4) in $\widetilde{\mathbb{H}}_{\text {sup }}^{n+}$, and for the $\mu$-almost everywhere convergence of $X^{\tau}(0)$ to this solution as $\tau \rightarrow \infty$, when $S=0$ in (A.1). 
Proposition A.1.4. Suppose that $(A, B)$ is stochastically stabilizable and consider $S=0$ in (A.1). Then there exists $X \in \widetilde{\mathbb{H}}_{\text {sup }}^{n+}$ such that $X^{\tau}(0, \ell) \rightarrow X(\ell)$ as $\tau \rightarrow \infty \mu$-almost everywhere in $\mathcal{S}$. Moreover, the control $\mathcal{S}$-coupled algebraic Riccati equations (A.4) are satisfied.

Proof. Consider $S=0$ in (A.1). Then it is easy to see that $X^{\tau-1}(0, \ell) \leq X^{\tau}(0, \ell)$ for all $\tau=1,2, \ldots$ Following the same arguments as in Proposition 6 in Costa and Fragoso (1995) it results, from the hypothesis that $(A, B)$ is stochastically stabilizable, that $E\left(x_{0}^{*} X\left(\theta_{0}\right) x_{0}\right) \leq d E\left(\left\|x_{0}\right\|^{2}\right)$ for some $d>0$, for any initial distribution $\widehat{\mu}$ satisfying Assumption 3.2.2 and $E\left(\left\|x_{0}\right\|^{2}\right)<\infty$. From Proposition A.1.1, it results that $X^{\tau}(0, \ell) \leq d I$ $\mu$-almost everywhere in $\mathcal{S}$, and thus $\sup \left\|X^{\tau}(0)\right\|_{\text {sup }} \leq d$. From Proposition A.1.2 there exists $X \in \widetilde{\mathbb{H}}_{\text {sup }}^{n+}$ such that $X^{\tau}(0, \ell) \stackrel{\tau}{\rightarrow} \rightarrow X(\ell)$ as $\tau \rightarrow \infty, \mu$-almost everywhere in $\mathcal{S}$, completing the proof.

The next result provides a condition, based on the concept of stochastic detectability (see Definition 5.2.4), to guarantee that any solution for the control $\mathcal{S}$-coupled algebraic Riccati equations (A.4) in $\widetilde{\mathbb{H}}_{\text {sup }}^{n+}$ will be a stabilizing solution. It follows similar reasoning as in Proposition 7 by Costa and Fragoso (1995).

Proposition A.1.5. Suppose that $(C, A)$ is stochastically detectable and $X \in \widetilde{\mathbb{H}}_{\text {sup }}^{n+}$ satisfies (A.4). Then $X$ is a stabilizing solution for (A.4).

Proof. The proof will follow several intermediate steps.

Step 1: It is going to be shown, for $X \in \widetilde{\mathbb{H}}_{\text {sup }}^{n+}$ satisfying (A.4), $K=-\mathcal{K}(X)$, and $d_{0}:=\frac{\|X\|_{\text {sup }}}{\epsilon_{D}}$, that

$$
E\left(\sum_{k=0}^{\infty}\|C(\theta(k)) x(k)\|^{2}+\|K(\theta(k)) x(k)\|^{2}\right) \leq d_{0} E\left(\left\|x_{0}\right\|^{2}\right) .
$$

Proof of Step 1: From (A.4) and setting $\widehat{u}(k)=K(\theta(k)) x(k)$ one gets that

$$
\begin{aligned}
X(\ell)= & A(\ell)^{*} \mathcal{E}(X)(\ell) A(\ell)+A(\ell)^{*} \mathcal{E}(X)(\ell) B(\ell) K(\ell)+C(\ell)^{*} C(\ell) \\
= & A(\ell)^{*} \mathcal{E}(X)(\ell) A(\ell)+A(\ell)^{*} \mathcal{E}(X)(\ell) B(\ell) K(\ell)+K(\ell)^{*} B(\ell)^{*} \mathcal{E}(X)(\ell) A(\ell) \\
& -K(\ell)^{*} B(\ell)^{*} \mathcal{E}(X)(\ell) A(\ell)+C(\ell)^{*} C(\ell) \\
= & A(\ell)^{*} \mathcal{E}(X)(\ell) A(\ell)+A(\ell)^{*} \mathcal{E}(X)(\ell) B(\ell) K(\ell)+K(\ell)^{*} B(\ell)^{*} \mathcal{E}(X)(\ell) A(\ell) \\
& +K(\ell)^{*} Q(\ell) K(\ell)+C(\ell)^{*} C(\ell) \\
= & A(\ell)^{*} \mathcal{E}(X)(\ell) A(\ell)+A(\ell)^{*} \mathcal{E}(X)(\ell) B(\ell) K(\ell)+K(\ell)^{*} B(\ell)^{*} \mathcal{E}(X)(\ell) A(\ell) \\
& +K(\ell)^{*} B(\ell)^{*} \mathcal{E}(X)(\ell) B(\ell) K(\ell)+K(\ell)^{*} D(\ell)^{*} D(\ell) K(\ell)+C(\ell)^{*} C(\ell) \\
= & (A(\ell)+B(\ell) K(\ell))^{*} \mathcal{E}(X)(\ell)(A(\ell)+B(\ell) K(\ell))+K(\ell)^{*} D(\ell)^{*} D(\ell) K(\ell) \\
& +C(\ell)^{*} C(\ell),
\end{aligned}
$$

and from the last expression and Proposition 4.2.2 one obtains that

$$
E\left(x(k)^{*} X(\theta(k)) x(k)\right)=E\left(x(k+1)^{*} X(\theta(k+1)) x(k+1)+\|D(\theta(k)) \widehat{u}(k)\|^{2}+\|C(\theta(k)) x(k)\|^{2}\right) .
$$


Iterating this equation, one gets that

$$
\begin{aligned}
E\left(x_{0}^{*} X\left(\theta_{0}\right) x_{0}\right) & =E\left(x(\tau)^{*} X(\theta(\tau)) x(\tau)\right)+\sum_{k=0}^{\tau-1} E\left(\|C(\theta(k)) x(k)\|^{2}+\|D(\theta(k)) \widehat{u}(k)\|^{2}\right) \\
& \geq \sum_{k=0}^{\tau-1} E\left(\|C(\theta(k)) x(k)\|^{2}+\|D(\theta(k)) \widehat{u}(k)\|^{2}\right) .
\end{aligned}
$$

Taking the limit as $\tau \rightarrow \infty$ and noticing that $0 \leq E\left(x_{0}^{*} X\left(\theta_{0}\right) x_{0}\right) \leq\|X\|_{\sup } E\left(\left\|x_{0}\right\|^{2}\right)$, and that $D(\ell)^{*} D(\ell) \geq \epsilon_{D} I \mu$-almost everywhere in $\mathcal{S}$, one obtains that

$$
\begin{aligned}
\|X\|_{\text {sup }} E\left(\left\|x_{0}\right\|^{2}\right) & \geq E\left(\sum_{k=0}^{\infty}\|C(\theta(k)) x(k)\|^{2}+\|D(\theta(k)) K(\theta(k)) x(k)\|^{2}\right) \\
& \geq E\left(\sum_{k=0}^{\infty}\|C(\theta(k)) x(k)\|^{2}+\epsilon_{D}\|K(\theta(k)) x(k)\|^{2}\right) \\
& \geq \epsilon_{D} E\left(\sum_{k=0}^{\infty}\|C(\theta(k)) x(k)\|^{2}+\|K(\theta(k)) x(k)\|^{2}\right),
\end{aligned}
$$

and (A.5) follows.

Consider now $\mathcal{L}$ as in (4.7) with $\Gamma(\ell)=A(\ell)+B(\ell) K(\ell)$. For the system (4.1) define the sequence $F(k, \ell)$ as in (4.12). It follows from Proposition 4.3.1 that $F(k)=\mathcal{L}^{k}(R)$. Since $(C, A)$ is stochastically detectable one can find $H \in \mathbb{H}_{\text {sup }}^{r, n}$ such that $r_{\sigma}(\widetilde{\mathcal{L}})<1$, where $\widetilde{\mathcal{L}}$ is as in $(4.7)$ with $\Gamma(\ell)=A(\ell)+H(\ell) C(\ell), \ell \in \mathcal{S}$. Choose $\epsilon>0$ such that $\left(1+\epsilon^{2}\right) r_{\sigma}(\widetilde{\mathcal{L}})<1$ and define $\widetilde{\mathcal{L}}_{\epsilon}=\left(1+\epsilon^{2}\right) \widetilde{\mathcal{L}}$, so that $r_{\sigma}\left(\widetilde{\mathcal{L}}_{\epsilon}\right)<1$. From Lemma 1 by Kubrusly $(1985)$ and the fact that $r_{\sigma}\left(\widetilde{\mathcal{L}}_{\epsilon}\right)<1$ it follows that there exist scalars $a>0$, and $0<b<1$ such that $\left\|\widetilde{\mathcal{L}}_{\epsilon}^{k}\right\| \leq a b^{k}$. Define $\mathcal{D}_{\epsilon} \in \mathbb{B}\left(\mathbb{H}_{1}^{n}\right)$ as follows, for $U \in \mathbb{H}_{1}^{n}$, set

$$
\mathcal{D}_{\epsilon}(U)(\ell)=\left(1+\frac{1}{\epsilon^{2}}\right) \int_{\mathcal{S}} g(t, \ell)(B(t) K(t)-H(t) C(t)) U(t)(B(t) K(t)-H(t) C(t))^{*} \mu(d t) .
$$

Define the sequence $M(k)$ in $\mathbb{H}_{1}^{n+}$ as follows, for $k \in \mathbb{N}, \ell \in \mathcal{S}$,

$$
\begin{aligned}
M(k+1, \ell) & =\widetilde{\mathcal{L}}_{\epsilon}(M(k))(\ell)+\mathcal{D}_{\epsilon}(F(k))(\ell) \\
M(0, \ell) & =R(\ell) .
\end{aligned}
$$

Step 2: $M(k) \geq F(k) \geq 0$.

Proof of Step 2: After some algebraic manipulations one can get that for any $U \in \mathbb{B}\left(\mathbb{H}_{1}^{n+}\right)$, $\mathcal{L}(U)(\ell) \leq \widetilde{\mathcal{L}}_{\epsilon}(U)(\ell)+\mathcal{D}_{\epsilon}(U)(\ell)$. It will be shown by induction on $k$ that $M(k) \geq F(k) \geq 0$. For $k=0$ it follows by definition (see (4.12), (A.6)) that $M(0)=R(0)=F(0)$. Suppose $M(k) \geq F(k)$. From (4.12), (A.6) and recalling that $\widetilde{\mathcal{L}}_{\epsilon}$ is a positive operator it results that

$$
\begin{aligned}
M(k+1, \ell) & =\widetilde{\mathcal{L}}_{\epsilon}(M(k))(\ell)+\mathcal{D}_{\epsilon}(F(k))(\ell) \\
& \geq \widetilde{\mathcal{L}}_{\epsilon}(F(k))(\ell)+\mathcal{D}_{\epsilon}(F(k))(\ell) \\
& \geq \mathcal{L}(F(k))(\ell) \\
& =F(k+1, \ell),
\end{aligned}
$$


completing the proof of Step 2.

Step 3: For some $\widehat{c}>0$ one has that $\sum_{i=0}^{\infty}\left\|\mathcal{D}_{\epsilon}(F(i))\right\|_{1} \leq \widehat{c} E\left(\left\|x_{0}\right\|^{2}\right)$.

Proof of Step 3: First it will be shown that

$$
\left\|\mathcal{D}_{\epsilon}(F(k))\right\|_{1} \leq c_{0}\left(E\left(\|C(\theta(k)) x(k)\|^{2}\right)+E\left(\|K(\theta(k)) x(k)\|^{2}\right)\right)
$$

where $c_{0}=2 c_{\epsilon} \max \left\{\|H\|_{\text {sup }}^{2},\|B\|_{\text {sup }}^{2}\right\}$, and $c_{\epsilon}=\left(1+\frac{1}{\epsilon^{2}}\right)$. From Fubini's theorem and from the definition of the operator $\mathcal{D}_{\epsilon}$ stated above, it follows that

$$
\begin{aligned}
\left\|\mathcal{D}_{\epsilon}(F(k))\right\|_{1}= & \int_{\mathcal{S}}\left\|\mathcal{D}_{\epsilon}(F(k))(\ell)\right\| \mu(d \ell) \\
= & \int_{\mathcal{S}} \| c_{\epsilon} \int_{\mathcal{S}} g(t, \ell)(B(t) K(t)-H(t) C(t)) F(k, t) \\
& (B(t) K(t)-H(t) C(t))^{*} \mu(d t) \| \mu(d \ell) \\
\leq & c_{\epsilon}\left(\|H\|_{\sup }^{2} \int_{\mathcal{S}}\left\|C(t) F(k, t) C(t)^{*}\right\| \mu(d t)\right. \\
& +\|B\|_{\text {sup }}^{2} \int_{\mathcal{S}}\left\|K(t) F(k, t) K(t)^{*}\right\| \mu(d t) \\
& \left.+2\|H\|_{\text {sup }}\|B\|_{\sup } \int_{\mathcal{S}}\left\|K(t) F(k, t) C(t)^{*}\right\| \mu(d t)\right),
\end{aligned}
$$

since $\int_{\mathcal{S}} g(t, \ell) \mu(d \ell)=1$. It will be shown now that

$$
\int_{\mathcal{S}}\left\|K(t) F(k, t) C(t)^{*}\right\| \mu(d t) \leq E(\|K(\theta(k)) x(k)\|\|C(\theta(k)) x(k)\|) .
$$

Noticing that $x(k)$ is $\mathcal{F}_{k-1}$-measurable, it follows that

$$
E\left(\|K(\theta(k)) x(k)\|\|C(\theta(k)) x(k)\| \mid \mathcal{F}_{k-1}\right)=\int_{\mathcal{S}}\|K(\ell) x(k)\|\|C(\ell) x(k)\| g(\theta(k-1), \ell) \mu(d \ell) .
$$

Then, recalling the definition of $F(k, t)$ in (4.12) it follows from Fubini's theorem that

$$
\begin{aligned}
E(\|K(\theta(k)) x(k)\|\|C(\theta(k)) x(k)\|) & =E\left(E\left(\|K(\theta(k)) x(k)\|\|C(\theta(k)) x(k)\| \mid \mathcal{F}_{k-1}\right)\right) \\
& =E\left(\int_{\mathcal{S}}\|K(\ell) x(k)\|\|C(\ell) x(k)\| g(\theta(k-1), \ell) \mu(d \ell)\right) \\
& =\int_{\mathcal{S}} E(\|K(\ell) x(k)\|\|C(\ell) x(k)\| g(\theta(k-1), \ell) \mu(d \ell)) \\
& \left.\geq \int_{\mathcal{S}}\left\|E\left(K(\ell) x(k) x(k)^{*} C(\ell)^{*} g(\theta(k-1), \ell)\right)\right\| \mu(d \ell)\right) \\
& =\int_{\mathcal{S}}\left\|K(\ell) E\left(x(k) x(k)^{*} g(\theta(k-1), \ell)\right) C(\ell)^{*}\right\| \mu(d \ell) \\
& =\int_{\mathcal{S}}\left\|K(\ell) F(k, \ell) C(\ell)^{*}\right\| \mu(d \ell) .
\end{aligned}
$$


Similar reasoning shows that

$$
\begin{aligned}
& \int_{\mathcal{S}}\left\|C(t) F(k, t) C(t)^{*}\right\| \mu(d t) \leq E\left(\|C(\theta(k)) x(k)\|^{2}\right), \text { and } \\
& \int_{\mathcal{S}}\left\|K(t) F(k, t) K(t)^{*}\right\| \mu(d t) \leq E\left(\|K(\theta(k)) x(k)\|^{2}\right) .
\end{aligned}
$$

From (A.9) it follows also that,

$$
\begin{aligned}
2 \int_{\mathcal{S}}\left\|K(\ell) F(k, \ell) C(\ell)^{*}\right\| \mu(d \ell) & \leq 2 E(\|K(\theta(k)) x(k)\|\|C(\theta(k)) x(k)\|) \\
& \leq E\left(\|K(\theta(k)) x(k)\|^{2}\right)+E\left(\|C(\theta(k)) x(k)\|^{2}\right) .
\end{aligned}
$$

Combining these inequalities into (A.8) yields to (A.7). From (A.5) and (A.7) it follows that Step 3 is satisfied with $\widehat{c}=c_{0} \frac{\|X\|_{\text {sup }}}{\epsilon_{D}}$.

Step 4: For $R$ as in (4.5), one gets that

$$
\sum_{k=0}^{\infty}\left\|\mathcal{L}^{k}(R)\right\|_{1} \leq \frac{a}{1-b}\|R\|_{1}+\frac{a}{1-b} \widehat{c} E\left(\left\|x_{0}\right\|^{2}\right)
$$

Proof of Step 4: From Step 2 it follows that $\|F(k)\|_{1} \leq\|M(k)\|_{1}$ and, from (A.6), that $M(k)=\mathcal{L}_{\epsilon}^{k}(R)+\sum_{i=0}^{k-1} \mathcal{L}_{\epsilon}^{k-1-i}\left(\mathcal{D}_{\epsilon}(F(i))\right)$. Recalling that $F(k)=\mathcal{L}^{k}(R)$ one gets that $\left\|\mathcal{L}^{k}(R)\right\|_{1} \leq a b^{k}\|R\|_{1}+\sum_{i=0}^{k-1} a b^{k-1-i}\left\|\mathcal{D}_{\epsilon}(F(i))\right\|_{1}$. From Step 3 it follows that

$$
\begin{aligned}
\sum_{k=0}^{\infty} \sum_{i=0}^{k-1} b^{k-1-i}\left\|\mathcal{D}_{\epsilon}(F(i))\right\|_{1} & =\left(\sum_{i=0}^{\infty}\left\|\mathcal{D}_{\epsilon}(F(i))\right\|_{1}\right)\left(\sum_{k=i+1}^{\infty} b^{k-1-i}\right) \\
& \leq \frac{1}{1-b} \widehat{c} E\left(\left\|x_{0}\right\|^{2}\right),
\end{aligned}
$$

and thus results the Step 4.

To conclude the proof of the proposition, the goal will be to show that for any $W \in \mathbb{H}_{1}^{n}$ it holds that $\sum_{k=0}^{\infty}\left\|\mathcal{L}^{k}(W)\right\|_{1}<\infty$ so that, from Lemma 1 in Kubrusly (1985), one can get that $r_{\sigma}(\mathcal{L})<1$. From Remark 3.1.1 there exist $W^{j} \in \mathbb{H}_{1}^{n+}, j=1,2,3,4$, such that $W=\left(W^{1}-W^{2}\right)+\sqrt{-1}\left(W^{3}-W^{4}\right)$. From linearity of $\mathcal{L}$ it results that $\sum_{k=0}^{\infty}\left\|\mathcal{L}^{k}(W)\right\|_{1} \leq$ $\sum_{j=1}^{4} \sum_{k=0}^{\infty}\left\|\mathcal{L}^{k}\left(W^{j}\right)\right\|_{1}$ and thus it is enough to show that $\sum_{k=0}^{\infty}\left\|\mathcal{L}^{k}(P)\right\|_{1}<\infty$ for any $P \in \mathbb{H}_{1}^{n+}$. Consider an arbitrary $P \in \mathbb{H}_{1}^{n+}$ and set $P^{r}(\ell)=\|P(\ell)\| I$. Clearly one has that $P(\ell) \leq$ $P^{r}(\ell)$ and $P^{r} \in \mathbb{H}_{1}^{n+}$. Since $\mathcal{L}$ is a positive operator it follows that $0 \leq \mathcal{L}^{k}(P) \leq \mathcal{L}^{k}\left(P^{r}\right)$ and therefore $\left\|\mathcal{L}^{k}(P)\right\|_{1} \leq\left\|\mathcal{L}^{k}\left(P^{r}\right)\right\|_{1}$. From the proof of Theorem 4.3 .3 ii) $\rightarrow$ iii), one can find an initial distribution $\widehat{\mu}$ satisfying Assumption 3.2.2 and $E\left(\left\|x_{0}\right\|^{2}\right)<\infty$ such that $R(\ell)$ as defined in (4.5) can be written as $R(\ell)=P^{r}(\ell)$. From Step 4 with $R=P^{r}$ 
it follows that $\sum_{k=0}^{\infty}\left\|\mathcal{L}^{k}(P)\right\|_{1} \leq \sum_{k=0}^{\infty}\left\|\mathcal{L}^{k}\left(P^{r}\right)\right\|_{1}<\infty$ for $P^{r}(\ell)=\|P(\ell)\| I$ and arbitrary $P \in \mathbb{H}_{1}^{n+}$, completing the proof.

The main result of this section is presented below in Theorem A.1.6, which guarantees the existence and uniqueness among the positive semi-definite solutions of the control $\mathcal{S}$-coupled algebraic Riccati equations. Furthermore it shows that this solution is the stabilizing one and the iterations of the operator $\mathcal{R}$ starting from any positive semidefinite element will converge $\mu$-almost everywhere to this solution.

Theorem A.1.6. If $(A, B)$ is stochastically stabilizable then:

(i) Consider $S=0$ in (A.1). Then there exists $X \in \widetilde{\mathbb{H}}_{\text {sup }}^{n+}$ satisfying (A.4) such that $X^{\tau}(0, \ell) \rightarrow X(\ell)$ as $\tau \rightarrow \infty \mu$-almost everywhere in $\mathcal{S}$.

If in addition $(C, A)$ is stochastically detectable then:

(ii) $X$ is the stabilizing solution for (A.4),

(iii) for any $S \in \widetilde{\mathbb{H}}_{\text {sup }}^{n+}$ and the sequence $Y^{\tau}(k)=\mathcal{R}\left(Y^{\tau}(k+1)\right), Y^{\tau}(\tau)=S$, it follows that $Y^{\tau}(0, \ell) \rightarrow X(\ell)$ as $\tau \rightarrow \infty \mu$-almost everywhere in $\mathcal{S}$, and

(iv) $X$ is the unique solution for (A.4) in $\widetilde{\mathbb{H}}_{\text {sup }}^{n+}$.

Proof. Each item is proved below.

Proof of (i): From Proposition A.1.4 it follows (i).

Proof of (ii): From Propositions A.1.5 it follows (ii).

Proof of (iii): Initially it will be shown that $Y^{\tau}(k, \ell) \geq X^{\tau}(k, \ell)$. Consider $k=\tau, \tau-1$, $\ldots, 0$. Indeed for $k=\tau$ the result is immediate since

$$
Y^{\tau}(\tau)=S \geq 0=X^{\tau}(\tau)
$$

Suppose it holds for $k+1$, that is, $Y^{\tau}(k+1)-X^{\tau}(k+1) \geq 0$. Setting

$$
\begin{aligned}
& F^{\tau}(k)=-\mathcal{K}\left(Y^{\tau}(k+1)\right) \\
& G^{\tau}(k)=-\mathcal{K}\left(X^{\tau}(k+1)\right),
\end{aligned}
$$

one gets, after some algebraic manipulations, that

$$
\begin{aligned}
& \left(Y^{\tau}(k, \ell)-X^{\tau}(k, \ell)\right) \\
& \quad\left(A(\ell)+B(\ell) F^{\tau}(k, \ell)\right)^{*} \mathcal{E}\left(Y^{\tau}(k+1)-X^{\tau}(k+1)\right)(\ell)\left(A(\ell)+B(\ell) F^{\tau}(k, \ell)\right) \\
& \quad+\left(F^{\tau}(k, \ell)-G^{\tau}(k, \ell)\right)^{*} \mathcal{Q}\left(X^{\tau}(k+1)\right)(\ell)\left(F^{\tau}(k, \ell)-G^{\tau}(k, \ell)\right) \\
& \quad \geq 0
\end{aligned}
$$

showing that indeed $Y^{\tau}(k, \ell) \geq X^{\tau}(k, \ell)$ for $k=\tau, \tau-1, \ldots, 0$. From Proposition A.1.2 it follows that there exist $Y_{s p}, Y_{i f} \in \widetilde{\mathbb{H}}_{\text {sup }}^{n+}$ such that $\lim \sup _{\tau \rightarrow \infty} Y^{\tau}(0, \ell)=Y_{s p}(\ell)$ and $\liminf _{\tau \rightarrow \infty} Y^{\tau}(0, \ell)=Y_{i f}(\ell) \mu$-almost everywhere in $\mathcal{S}$. Since $Y^{\tau}(0) \geq X^{\tau}(0)$ and $\lim _{\tau \rightarrow} X^{\tau \rightarrow \infty}(0, \ell)=X(\ell)$ one concludes that $\mu$-almost everywhere in $\mathcal{S}, Y_{i f}(\ell) \geq X(\ell)$, and 
thus that $E\left(x_{0}^{*} Y_{i f}\left(\theta_{0}\right) x_{0}\right) \geq E\left(x_{0}^{*} X\left(\theta_{0}\right) x_{0}\right)$ for any initial distribution $\widehat{\mu}$ satisfying Assumption 3.2.2 and $E\left(\left\|x_{0}\right\|^{2}\right)<\infty$. On the other hand, setting $K=-\mathcal{K}(X)$ and noticing that $Y^{\tau}(k+1)=Y^{\tau-1}(k)$ it follows, after some algebraic manipulations, that

$$
\begin{aligned}
Y^{\tau}(0, \ell) \leq K(\ell)^{*} D(\ell)^{*} D(\ell) K(\ell)+C(\ell)^{*} C(\ell) \\
+(A(\ell)+B(\ell) K(\ell))^{*} \mathcal{E}\left(Y^{\tau-1}(0)\right)(\ell)(A(\ell)+B(\ell) K(\ell)) .
\end{aligned}
$$

From Proposition A.1.2 and Fatou's lemma (see Hernández-Lerma and Lasserre, 2003, Section 1.5.2) it follows, $\mu$-almost everywhere in $\mathcal{S}$, that

$$
\begin{aligned}
x^{*} Y_{s p}(\ell) x= & \limsup _{\tau \rightarrow \infty} x^{*} Y_{0}^{\tau}(\ell) x \\
\leq & x^{*}\left(K(\ell)^{*} D(\ell)^{*} D(\ell) K(\ell)+C(\ell)^{*} C(\ell)\right. \\
& \left.+(A(\ell)+B(\ell) K(\ell))^{*} \mathcal{E}\left(Y_{s p}\right)(\ell)(A(\ell)+B(\ell) K(\ell))\right) x
\end{aligned}
$$

and thus

$$
\begin{aligned}
Y_{s p}(\ell) \leq & K(\ell)^{*} D(\ell)^{*} D(\ell) K(\ell)+C(\ell)^{*} C(\ell) \\
& +(A(\ell)+B(\ell) K(\ell))^{*} \mathcal{E}\left(Y_{s p}\right)(\ell)(A(\ell)+B(\ell) K(\ell)) .
\end{aligned}
$$

Set $\widehat{u}(k)=K(\theta(k)) x(k)=-\mathcal{K}(X)(\theta(k)) x(k)$. Then it follows that

$$
\begin{aligned}
E\left(x(k)^{*} Y_{s p}(\theta(k)) x(k)\right) \leq & E\left(\|C(\theta(k)) x(k)\|^{2}+\|D(\theta(k)) \widehat{u}(k)\|^{2}\right. \\
& \left.+x(k+1)^{*} Y_{s p}(\theta(k+1)) x(k+1)\right) .
\end{aligned}
$$

Iterating this equation one obtains that

$$
E\left(x_{0}^{*} Y_{s p}\left(\theta_{0}\right) x_{0}\right) \leq E\left(x(\tau)^{*} Y_{s p}(\theta(\tau)) x(\tau)\right)+\sum_{k=0}^{\tau-1} E\left(\left\|C\left(\theta_{0}\right) x(k)\right\|^{2}+\|D(\ell) \widehat{u}(k)\|^{2}\right)
$$

so that, taking the limit as $\tau \rightarrow \infty$, one has that

$$
E\left(x_{0}^{*} Y_{s p}\left(\theta_{0}\right) x_{0}\right) \leq J\left(x_{0}, \theta_{0}, \widehat{u}\right)=E\left(x_{0}^{*} X\left(\theta_{0}\right) x_{0}\right) .
$$

From Proposition A.1.2 one gets that

$$
\begin{aligned}
E\left(x_{0}^{*} X\left(\theta_{0}\right) x_{0}\right) & \leq E\left(x_{0}^{*} Y_{i f}\left(\theta_{0}\right) x_{0}\right)=E\left(\liminf _{\tau \rightarrow \infty} x_{0}^{*} Y^{\tau}\left(0, \theta_{0}\right) x_{0}\right) \\
& \leq E\left(\limsup _{\tau \rightarrow \infty} x_{0}^{*} Y^{\tau}\left(0, \theta_{0}\right) x_{0}\right)=E\left(x_{0}^{*} Y_{s p}\left(\theta_{0}\right) x_{0}\right) \leq E\left(x_{0}^{*} X\left(\theta_{0}\right) x_{0}\right)
\end{aligned}
$$

and thus

$$
E\left(x_{0}^{*} Y_{i f}\left(\theta_{0}\right) x_{0}\right)=E\left(x_{0}^{*} Y_{s p}\left(\theta_{0}\right) x_{0}\right)=E\left(x_{0}^{*} X\left(\theta_{0}\right) x_{0}\right) .
$$

From Proposition A.1.1 it follows (iii).

Proof of (iv): It is easy to see that (iii) implies (iv) (if $\widetilde{X}$ is another solution for (A.4) in $\widetilde{\mathbb{H}}_{\text {sup }}^{n+}$ then just take $S=\widetilde{X}$ so that from (iii) one has that $Y^{\tau}(0)=\widetilde{X}=X \mu$-almost everywhere in $\mathcal{S}$ ).

Notice that (i) and (iii) in Theorem A.1.6 could be alternatively rewritten respectively as $\mathcal{R}^{\tau}(0)(\ell) \rightarrow X(\ell)$ and $\mathcal{R}^{\tau}(S)(\ell) \rightarrow X(\ell)$ as $\tau \rightarrow \infty \mu$-almost everywhere in $\mathcal{S}$ since that, by iterating the backward recursive equations for $X^{\tau}(k)$ as in (A.1) with $S=0$ and $Y^{\tau}(k)=\mathcal{R}\left(Y^{\tau}(k+1)\right), Y^{\tau}(\tau)=S$, one would get that $X^{\tau}(0)=\mathcal{R}^{\tau}(0)$ and $Y^{\tau}(0)=\mathcal{R}^{\tau}(S)$. 


\section{A.2 Filtering $\mathcal{S}$-Coupled Algebraic Riccati Equations}

This appendix presents the conditions for the existence and uniqueness of a stabilizing positive semi-definite solution to the filtering $\mathcal{S}$-coupled algebraic Riccati equations. In Section A.2.1 the filtering $\mathcal{S}$-coupled algebraic Riccati equations are introduced. Some useful auxiliary results are stated in Section A.2.2. Finally in Section A.2.3 the conditions for the existence and uniqueness of a stabilizing positive semi-definite solution to the filtering $\mathcal{S}$-coupled algebraic Riccati equations are presented in Theorem A.2.8. These results are applied in Sections 6.2 and 7.2 in the design of filters to address infinite-horizon problems.

\section{A.2.1 Filtering $\mathcal{S}$-Coupled Algebraic Riccati Equations}

In this section it is considered the same hypotheses assumed in Section 6.2. In particular it is assumed that $\{\theta(k) ; k \in \mathbb{N}\}$ is uniformly ergodic as stated in Assumption 6.2.1. Moreover, the sequence $\{\pi(k) ; k \in \mathbb{N}\}$ is defined as in (6.41). In what follows, for a $\sigma$ finite measure $\vartheta$ on $\mathcal{S}$ and a real function $f: \mathcal{S} \rightarrow \mathbb{R}$ define $\vartheta f:=\int_{\mathcal{S}} f(s) \vartheta(d s)$ provided that the integral exists.

From Assumption 6.2.1 there exists an invariant probability measure $\vartheta$ for $\mathcal{G}(\cdot, \cdot)$ and $\beta_{1} \geq 0,0<\rho_{1}<1$ such that

$$
\sup _{\ell \in \mathcal{S}}\left\|\mathcal{G}^{k}(\ell, \cdot)-\vartheta(\cdot)\right\| \leq \beta_{1} \rho_{1}^{k}
$$

where $\left\|\mathcal{G}^{k}(\ell, \cdot)-\vartheta(\cdot)\right\|=\sup _{\|f\|_{\text {sup }} \leq 1}\left\|\mathcal{G}^{k} f(\ell)-\vartheta f\right\|$. Since $\vartheta$ is an invariant probability measure, for any $C \in \mathcal{B}(\mathcal{S})$,

$$
\vartheta(C)=\int_{\mathcal{S}} \mathcal{G}(\ell, C) \vartheta(d \ell)=\int_{\mathcal{S}} \int_{C} g(\ell, t) \mu(d t) \vartheta(d \ell)
$$

it follows that $\mu \gg \vartheta$. From this, one can conclude that there exists $\pi \in \mathbb{H}_{1}^{1+}$ such that $\frac{d \vartheta}{d \mu}=\pi$, that is, $\vartheta(B)=\int_{B} \pi(\ell) \mu(d \ell)$ (thus $\pi(\ell) \in \mathbb{R}^{+}$). The next proposition states that $\pi$ is an invariant probability density for $\mathcal{G}(\cdot, \cdot)$.

Proposition A.2.1. From Assumption 6.2.1 it follows that $\pi \in \mathbb{H}_{1}^{1+} \cap \mathbb{H}_{\text {sup }}^{1+}$ and satisfies

$$
\pi(\ell)=\int_{\mathcal{S}} g(s, \ell) \pi(s) \mu(d s) \text { and }\|\pi\|_{1}=1 .
$$

Furthermore, for $k \in \mathbb{N}, k \geq 1$, and some $\beta_{1}^{\prime}>0$ (recall the notation for $\pi(k)$ in Remark 3.1.5, that is, $\pi(k)$ below means $\pi(k, \ell), \ell \in \mathcal{S})$,

$$
\|\pi(k)-\pi\|_{1} \leq \beta_{1} \rho_{1}^{k} \text { and }\|\pi(k)-\pi\|_{\text {sup }} \leq \beta_{1}^{\prime} \rho_{1}^{k} .
$$

Proof. Set $\mu_{\pi} f=\int_{\mathcal{S}} f(\ell) \pi(\ell) \mu(d \ell)$. For any $f \in \mathbb{H}_{\text {sup }}^{1}$,

$$
\int_{\mathcal{S}} \mathcal{G}^{k} f(\ell) \nu(\ell) \mu(d \ell)=\left\langle\nu ; \mathcal{G}^{k} f\right\rangle=\left\langle T^{k} \nu ; f\right\rangle \leq \int_{S}\left\{\mu_{\pi} f+\beta_{1} \rho_{1}^{k}\right\} \nu(\ell) \mu(d \ell)
$$




$$
=\|\nu\|_{1}\left\{\mu_{\pi} f+\beta_{1} \rho_{1}^{k}\right\}=\mu_{\pi} f+\beta_{1} \rho_{1}^{k}=\langle\pi ; f\rangle+\beta_{1} \rho_{1}^{k},
$$

since $\|\nu\|_{1}=1$. That is, $\left\langle T^{k} \nu-\pi ; f\right\rangle \leq \beta_{1} \rho_{1}^{k}$ for any $f \in \mathbb{H}_{\text {sup }}^{1}$, and thus $\mid\left\langle T^{k} \nu-\right.$ $\pi ; f\rangle|=| \int_{S}\left(T^{k} \nu(\ell)-\pi(\ell)\right) f(\ell) \mu(d \ell) \mid \leq \beta_{1} \rho_{1}^{k}$. Consider $B^{+}=\left\{\ell \in \mathcal{S} ; T^{k} \nu(\ell) \geq \pi(\ell)\right\}$, $B^{-}=\left\{\ell \in \mathcal{S} ; T^{k} \nu(\ell)<\pi(\ell)\right\}$ and $f(\ell)=1_{B^{+}}(\ell)-1_{B^{-}}(\ell)$. It follows that

$$
\left\|T^{k} \nu-\pi\right\|_{1}=\int_{\mathcal{S}}\left|T^{k} \nu(\ell)-\pi(\ell)\right| \mu(d \ell)=\int_{\mathcal{S}}\left(T^{k} \nu(\ell)-\pi(\ell)\right) f(\ell) \mu(d \ell) \leq \beta_{1} \rho_{1}^{k} .
$$

Notice that $\int_{\mathcal{S}}|T \pi(\ell)-\pi(\ell)| \mu(d \ell) \leq \int_{\mathcal{S}}\left|T\left(T^{k-1} \nu-\pi\right)(\ell)\right| \mu(d \ell)+\left\|T^{k} \nu-\pi\right\|_{1}$ and that $\int_{\mathcal{S}}\left|T\left(T^{k-1} \nu-\pi\right)(\ell)\right| \mu(d \ell) \leq \int_{\mathcal{S}} \int_{\mathcal{S}}\left|T^{k-1} \nu(t)-\pi(t)\right| g(t, \ell) \mu(d \ell) \mu(d t)=\left\|T^{k-1} \nu-\pi\right\|_{1}$. Combining the results, it follows that $\int_{\mathcal{S}}|T \pi(\ell)-\pi(\ell)| \mu(d \ell) \leq 2 \beta_{1} \rho_{1}^{k-1} \rightarrow 0$ as $k \rightarrow \infty$, so that $\int_{\mathcal{S}}|T \pi(\ell)-\pi(\ell)| \mu(d \ell)=0$, showing that $\pi=T \pi$. From Proposition 6.2.4 it holds that $\|\pi(k)\|_{1}=1$, and thus, by taking the limit as $k \rightarrow \infty$, one gets that $\|\pi\|_{1}=1$. Now it will be shown that $\left\|T^{k} \nu-\pi\right\|_{\text {sup }} \leq \beta_{1}^{\prime} \rho_{1}^{k}$ for some $\beta_{1}^{\prime}>0$. Notice that

$$
\begin{aligned}
\left|T^{k} \nu(\ell)-T^{k+m} \nu(\ell)\right| & =\left|T^{k}\left(\nu-T^{m} \nu\right)(\ell)\right|=\left|\int_{S} T^{k-1}\left(\nu-T^{m} \nu\right)(t) g(t, \ell) \mu(d t)\right| \\
& \leq c\left\{\int_{S}\left|T^{k-1} \nu(t)-\pi(t)\right| \mu(d t)+\int_{S}\left|T^{k+m-1} \nu(t)-\pi(t)\right| \mu(d t)\right\} \\
& \leq 2 c \beta_{1} \rho_{1}^{k-1}\left(1+\rho_{1}^{m}\right) \stackrel{k, m \rightarrow \infty}{\longrightarrow} 0 .
\end{aligned}
$$

Thus $\left\|T^{k} \nu-T^{k+m} \nu\right\|_{\text {sup }} \stackrel{k, m \rightarrow \infty}{\longrightarrow} 0$ and $\left\{T^{k} \nu\right\}$ is a Cauchy sequence in $\mathbb{H}_{\text {sup }}^{1}$. Since $\mathbb{H}_{\text {sup }}^{1}$ is a Banach space, it follows that $T^{k} \nu \rightarrow \tilde{\pi} \in \mathbb{H}_{\text {sup }}^{1}$, that is, $\left\|T^{k} \nu-\tilde{\pi}\right\|_{\text {sup }} \stackrel{k \rightarrow \infty}{\longrightarrow} 0$. Notice that

$$
\begin{aligned}
|\pi(\ell)-\tilde{\pi}(\ell)| & =\left|\pi(\ell)-T^{k} \nu(\ell)+T^{k} \nu(\ell)-\tilde{\pi}(\ell)\right| \\
& \leq c \int_{S}\left|T^{k-1} \nu(t)-\pi(t)\right| \mu(d t)+\left|T^{k} \nu(\ell)-\tilde{\pi}(\ell)\right| \\
& \leq c \beta_{1} \rho_{1}^{k-1}+\left|T^{k} \nu(\ell)-\tilde{\pi}(\ell)\right| \stackrel{k \rightarrow \infty}{\longrightarrow} 0
\end{aligned}
$$

and then $\pi=\tilde{\pi}$. Furthermore $\left|T^{k} \nu(\ell)-\pi(\ell)\right|=\left|\int_{S}\left(T^{k-1} \nu(t)-\tilde{\pi}(t)\right) g(t, \ell) \mu(d t)\right| \leq$ $c \int_{S}\left|T^{k-1} \nu(t)-\pi(t)\right| \mu(d t) \leq c \beta_{1} \rho_{1}^{k-1}$, completing the proof of the proposition.

It is well known that, for the case with no jumps, the Kalman filter is obtained iteratively from the so-called filtering Riccati difference equations and that, under some stabilizability and detectability conditions, these recursive solutions will converge to the (unique) stabilizing solution of the so-called filtering algebraic Riccati equation (see Davis and Vinter, 1985, Chapter 3). This section presents some results that trace a parallel with this general filtering theory.

The filtering $\mathcal{S}$-coupled Riccati difference equations are presented in (A.11) and (A.12), recursively for $k \in \mathbb{N}$, and in (A.13) and (A.14) the filtering $\mathcal{S}$-coupled algebraic Riccati equations are introduced. In Section A.2.2 some stability results are derived. These 
results are used in Section A.2.3 to show the existence of the stabilizing solution of the filtering $\mathcal{S}$-coupled algebraic Riccati equations and the convergence of the solution of the recursive filtering $\mathcal{S}$-coupled Riccati difference equations to this solution.

Next, the filtering $\mathcal{S}$-coupled Riccati difference equations are presented. For $k \in \mathbb{N}$ and $\ell \in \mathcal{S}$ set

$$
\begin{aligned}
U(k, \ell):= & \left(H(\ell) H(\ell)^{*} \pi(k, \ell)+L(\ell) Y(k, \ell) L(\ell)^{*}\right)^{-1}, \\
Y(k+1, \ell):= & \int_{\mathcal{S}}\left[A(s) Y(k, s) A(s)^{*}+G(s) G(s)^{*} \pi(k, s)\right. \\
& \left.-A(s) Y(k, s) L(s)^{*} U(k, s) L(s) Y(k, s) A(s)^{*}\right] g(s, \ell) \mu(d s),
\end{aligned}
$$

where $Y(0) \in \mathbb{H}_{1}^{n+} \cap \mathbb{H}_{\text {sup }}^{n+}$. The filtering $\mathcal{S}$-coupled algebraic Riccati equations are defined as follows,

$$
\begin{aligned}
U(\ell):= & \left(H(\ell) H(\ell)^{*} \pi(\ell)+L(\ell) Y(\ell) L(\ell)^{*}\right)^{-1}, \\
Y(\ell):= & \int_{\mathcal{S}}\left[A(s) Y(s) A(s)^{*}+G(s) G(s)^{*} \pi(s)\right. \\
& \left.-A(s) Y(s) L(s)^{*} U(s) L(s) Y(s) A(s)^{*}\right] g(s, \ell) \mu(d s) .
\end{aligned}
$$

Notice that from Assumption 6.2.1 and (A.10) it results that $H(\ell) H(\ell)^{*} \pi(\ell) \geq \epsilon_{H}(\ell) I$.

Set $\mathbb{K}=\left\{K(k) \in \mathbb{H}_{\text {sup }}^{p, n}, k \in \mathbb{N}\right\}$. For $V \in \mathbb{H}_{1}^{n+} \cap \mathbb{H}_{\text {sup }}^{n+}, K \in \mathbb{H}_{\text {sup }}^{p, n}, \mathcal{K}=$ $(K(0), K(1), \ldots) \in \mathbb{K}, \alpha \in \mathbb{H}_{1}^{1+} \cap \mathbb{H}_{\text {sup }}^{1+}, \beta \in \mathbb{H}_{1}^{1+}$, and $k \in \mathbb{N}$, recall the definition of the operators $\mathcal{L}(K, V), \mathcal{I}(K, V, \alpha), \mathcal{U}(K, V, \alpha), \mathcal{U}(V, \alpha), \mathcal{M}(V, \beta)$, stated in (6.8)-(6.12) and reproduced below,

$$
\begin{aligned}
\mathcal{L}(K, V)(\ell) & :=\int_{\mathcal{S}} g(s, \ell)(A(s)+K(s) L(s)) V(s)(A(s)+K(s) L(s))^{*} \mu(d s), \\
\mathcal{I}(K, V, \alpha)(\ell) & :=\int_{\mathcal{S}} g(s, \ell)\left[\alpha(s)\left((K(s) H(s))(K(s) H(s))^{*}+G(s) G(s)^{*}\right)+V(s)\right] \mu(d s), \\
\mathcal{U}(K, V, \alpha)(\ell) & :=\mathcal{L}(K, V)(\ell)+\mathcal{I}(K, 0, \alpha)(\ell), \\
\mathcal{M}(V, \beta)(\ell) & :=-A(\ell) V(\ell) L(\ell)^{*}\left(L(\ell) V(\ell) L(\ell)^{*}+\beta(\ell) H(\ell) H(\ell)^{*}\right)^{-1}, \\
\mathcal{U}(V, \alpha)(\ell) & :=\mathcal{U}(\mathcal{M}(V, \alpha), V, \alpha)(\ell),
\end{aligned}
$$

and define the sequences $Y(\mathcal{K}, V, k)$ and $Y(V, k)$ such that

$$
\begin{aligned}
Y(\mathcal{K}, V, k+1) & :=\mathcal{U}(K(k), Y(\mathcal{K}, V, k), \pi(k)), \quad Y(\mathcal{K}, V, 0):=V \\
Y(V, k+1) & :=\mathcal{U}(Y(V, k), \pi(k)), \quad Y(V, 0):=V .
\end{aligned}
$$

Remark A.2.2. For $V \in \mathbb{H}_{1}^{n+} \cap \mathbb{H}_{\text {sup }}^{n+}, Y(V, k)$ defined in (A.21) coincides with the sequence presented in (A.12) with $Y(0)=V$. Notice that in Chapter 6 the filtering $\mathcal{S}$ coupled Riccati difference equations and the filtering $\mathcal{S}$-coupled algebraic Riccati equations are defined in (6.21) and (6.51), respectively, based on this identity. Notice also that from Proposition 6.1.14 and recalling that operators $\mathcal{L}$ and $\mathcal{I}$ defined respectively in (A.15) and (A.16) are positive operators it follows that $Y(V, k) \in \mathbb{H}_{1}^{n+} \cap \mathbb{H}_{\text {sup }}^{n+}$, for $k \in \mathbb{N}$. 
The solution to the infinite-horizon filtering problem, stated in Problem 6.2.6, is related to the existence of stabilizing solutions $Y \in \mathbb{H}_{1}^{n+} \cap \mathbb{H}_{\text {sup }}^{n+}$ to the filtering $\mathcal{S}$-coupled algebraic Riccati equations (A.13)-(A.14) as defined next.

Definition A.2.3. $Y$ is a stabilizing solution for (A.13)-(A.14) if $Y \in \mathbb{H}_{1}^{n+} \cap \mathbb{H}_{\text {sup }}^{n+}$, and

$$
M(\ell)=\mathcal{M}(Y, \pi)=-A(\ell) Y(\ell) L(\ell)^{*}\left(H(\ell) H(\ell)^{*} \pi(\ell)+L(\ell) Y(\ell) L(\ell)^{*}\right)^{-1}
$$

stochastically stabilizes $(L, A)$ in the sense of Definition 5.2.4.

The next proposition is useful when studying the convergence of (A.12) to (A.14).

Proposition A.2.4. For $K \in \mathbb{H}_{\text {sup }}^{p, n}$ it holds that the sequence $(\mathcal{I}(K, 0, \pi(0)), \mathcal{I}(K, 0, \pi(1))$, ...) is Cauchy summable in $\mathbb{H}_{1}^{n}$, where, from (A.16),

$$
\mathcal{I}(K, 0, \pi(k))(\ell):=\int_{\mathcal{S}} g(s, \ell) \pi(k, s)\left[(K(s) H(s))(K(s) H(s))^{*}+G(s) G(s)^{*}\right] \mu(d s) .
$$

Proof. From (A.10) it follows that $\|\pi-\pi(k)\|_{1} \leq \beta_{1} \rho_{1}^{k}$. Then from (A.16) one gets

$$
\begin{aligned}
& \sum_{k=0}^{\infty} \sup _{\tau \in \mathbb{N}}\|\mathcal{I}(K, 0, \pi(k+\tau))-\mathcal{I}(K, 0, \pi(k))\|_{1} \\
& \left.\left.\leq \sum_{k=0}^{\infty} \sup _{\tau \in \mathbb{N}} \| \mathcal{I}(K, 0, \pi(k+\tau))-\mathcal{I}(K, 0, \pi)\right)\left\|_{1}+\right\| \mathcal{I}(K, 0, \pi)\right)-\mathcal{I}(K, 0, \pi(k)) \|_{1} \\
& \leq\left(\|K\|_{\text {sup }}^{2}\|H\|_{\text {sup }}^{2}+\|G\|_{\text {sup }}^{2}\right) \sum_{k=0}^{\infty} \sup _{\tau \in \mathbb{N}}\left(\beta_{1} \rho_{1}^{k+1}\left(1+\rho_{1}^{\tau}\right)\right) \\
& \leq\left(\|K\|_{\text {sup }}^{2}\|H\|_{\text {sup }}^{2}+\|G\|_{\text {sup }}^{2}\right) \frac{2 \beta_{1} \rho_{1}}{1-\rho_{1}} \\
& <\infty
\end{aligned}
$$

completing the proof.

The next result follows similar arguments as in Proposition 3 in Costa (1995).

Proposition A.2.5. If $V, W \in \mathbb{H}_{1}^{n+} \cap \mathbb{H}_{\text {sup }}^{n+}, \alpha, \beta \in \mathbb{H}_{1}^{1+} \cap \mathbb{H}_{\text {sup }}^{1+}$ and $V \geq W, \alpha \geq \beta>0$ then $\mathcal{U}(V, \alpha) \geq \mathcal{U}(W, \beta)$. Moreover for any $\mathcal{K} \in \mathbb{K}$ and $k \in \mathbb{N}, 0 \leq Y(V, k) \leq Y(\mathcal{K}, V, k)$.

Proof. For any $K \in \mathbb{H}_{\text {sup }}^{p, n}$ it follows from (A.17), (A.19) and Remark 6.1.15 that

$$
\begin{aligned}
\mathcal{U}(W, \beta)(\ell) & \leq \int_{\mathcal{S}} g(s, \ell)(A(s)+K(s) L(s)) V(s)(A(s)+K(s) L(s))^{*} \mu(d s) \\
& +\int_{\mathcal{S}} g(s, \ell) \alpha(s)\left(K(s) H(s) H(s)^{*} K(s)^{*}+G(s) G(s)^{*}\right) \mu(d s) \\
& =\mathcal{U}(K, V, \alpha)(\ell) .
\end{aligned}
$$

That is, for any $K \in \mathbb{H}_{\text {sup }}^{p, n}$ it holds

$$
\mathcal{U}(K, V, \alpha) \geq \mathcal{U}(\mathcal{M}(W, \beta), W, \beta)=\mathcal{U}(W, \beta)
$$


and in particular for $K=\mathcal{M}(V, \alpha)$ one can get that

$$
\mathcal{U}(\mathcal{M}(V, \alpha), V, \alpha)=\mathcal{U}(V, \alpha) \geq \mathcal{U}(\mathcal{M}(W, \beta), W, \beta)=\mathcal{U}(W, \beta) .
$$

It still must be shown that for any $\mathcal{K} \in \mathbb{K}$ and $k \in \mathbb{N}, 0 \leq Y(V, k) \leq Y(\mathcal{K}, V, k)$. It will be shown by induction on $k$. For $k=0$ it follows that $V=Y(V, 0)=Y(\mathcal{K}, V, 0)$. Assume now that the result holds for $k>0$. Then one gets that

$$
Y(\mathcal{K}, V, k) \geq Y(V, k) \geq 0 .
$$

From (A.22) it follows that

$$
Y(\mathcal{K}, V, k+1)=\mathcal{U}(K(k), Y(\mathcal{K}, V, k), \pi(k)) \geq \mathcal{U}(Y(V, k), \pi(k))=Y(V, k+1),
$$

completing the proof.

\section{A.2.2 Auxiliary Results}

In this subsection some stability results are presented. These results will be needed to demonstrate the main result for the stationary case in the next subsection. The first result is established in Proposition A.2.6 below.

Proposition A.2.6. Consider $\mathcal{L}$ as defined in (4.7). If $r_{\sigma}(\mathcal{L})<1$ then for any $V \in \mathbb{H}_{1}^{n+}$ there exists a unique $S \in \mathbb{H}_{1}^{n+}$ such that

$$
S-\mathcal{L}(S)=V
$$

Moreover if $V \in \mathbb{H}_{1}^{n+} \cap \mathbb{H}_{\text {sup }}^{n+}$ then $S \in \mathbb{H}_{1}^{n+} \cap \mathbb{H}_{\text {sup }}^{n+}$.

Proof. From the fact that $r_{\sigma}(\mathcal{L})<1$ it follows that $(I-\mathcal{L})^{-1}=\sum_{k=0}^{\infty} \mathcal{L}^{k}$ is a positive well defined operator in $\mathbb{B}\left(\mathbb{H}_{1}^{n}\right)$ and existence and uniqueness easily follow from this (see Weidmann (1980)). Notice now that from (4.7), Assumption 6.1.7, and for $k \geq 1$,

$$
\begin{aligned}
\left\|\mathcal{L}^{k}(V)(\ell)\right\| & \leq\|\Gamma\|_{\text {sup }}^{2} \int_{\mathcal{S}} g(t, \ell)\left\|\mathcal{L}^{k-1}(V)(t)\right\| \mu(d t) \\
& \leq c\|\Gamma\|_{\sup }^{2} \int_{\mathcal{S}}\left\|\mathcal{L}^{k-1}(V)(t)\right\| \mu(d t) \\
& =c\|\Gamma\|_{\text {sup }}^{2}\left\|\mathcal{L}^{k-1}(V)\right\|_{1} .
\end{aligned}
$$

From the fact that $r_{\sigma}(\mathcal{L})<1$, one can find real numbers $a>0,0<b<1$ such that $\left\|\mathcal{L}^{k}\right\| \leq a b^{k}$ (see Lemma 1 in Kubrusly (1985)) and therefore $\left\|\mathcal{L}^{k-1}(V)\right\|_{1} \leq a b^{k-1}\|V\|_{1}$. Thus, from (A.24) it results that

$$
\begin{aligned}
\|S(\ell)\| & =\left\|\sum_{k=0}^{\infty} \mathcal{L}^{k}(V)(\ell)\right\| \\
& \leq\|V(\ell)\|+\sum_{k=1}^{\infty}\left\|\mathcal{L}^{k}(V)(\ell)\right\| \\
& \leq\|V(\ell)\|+a c\|\Gamma\|_{\text {sup }}^{2}\|V\|_{1} \frac{1}{1-b} .
\end{aligned}
$$

From (A.25) one have that $\|S\|_{\text {sup }} \leq\|V\|_{\text {sup }}+a c\|\Gamma\|_{\text {sup }}^{2}\|V\|_{1} \frac{1}{1-b}$ showing that indeed $S \in \mathbb{H}_{1}^{n+} \cap \mathbb{H}_{\text {sup }}^{n+}$ whenever $V \in \mathbb{H}_{1}^{n+} \cap \mathbb{H}_{\text {sup }}^{n+}$. 
In the next proposition the definitions that follow are needed. For $K, F \in \mathbb{H}_{1}^{p, n}$ define the operators $\mathcal{L}^{K}, \mathcal{T}^{K}$ as in (4.7), (4.8) with $\Gamma(t)=A(t)+K(t) L(t)$ and $\mathcal{L}^{F}, \mathcal{T}^{F}$ as in (4.7), (4.8) with $\Gamma(t)=A(t)+F(t) L(t)$. Also recall that $\left(\mathcal{T}^{K}\right)^{*}=\mathcal{L}^{K}$ and $\left(\mathcal{T}^{F}\right)^{*}=\mathcal{L}^{F}$.

Proposition A.2.7. Suppose that $r_{\sigma}\left(\mathcal{L}^{F}\right)<1$ for some $F \in \mathbb{H}_{1}^{p, n}$. If there exists $K \in$ $\mathbb{H}_{1}^{p, n}, P \in \mathbb{H}_{1}^{n+}$, and $\alpha \in \mathbb{H}_{1}^{1+}, \alpha(\ell)>0, \ell \in \mathcal{S}$, satisfying

$$
P-\mathcal{L}^{K}(P) \geq S
$$

where $S \in \mathbb{H}_{1}^{n+}$ is defined as $S(\ell)=\int_{\mathcal{S}} g(s, \ell) \alpha(s)(K(s)-F(s))(K(s)-F(s))^{*} \mu(d s)$, then $r_{\sigma}\left(\mathcal{L}^{K}\right)<1$

Proof. From Lemma 3.1.4 there exists $V \in \mathbb{H}_{\text {sup }}^{n+}$ such that $\mathcal{T}^{K}(V)=r_{\sigma}\left(\mathcal{L}^{K}\right) V$. From this, (3.3) and (A.26) it results that

$$
\begin{aligned}
0 & \leq \int_{\mathcal{S}} \alpha(s) \operatorname{tr}\left((K(s)-F(s))^{*} \mathcal{E}(V)(s)(K(s)-F(s))\right) \mu(d s) \\
& =\int_{\mathcal{S}} \alpha(s) \operatorname{tr}\left((K(s)-F(s))(K(s)-F(s))^{*} \mathcal{E}(V)(s)\right) \mu(d s) \\
& =\int_{\mathcal{S}} \operatorname{tr}\left(\left(\int_{\mathcal{S}} g(s, \ell) \alpha(s)(K(s)-F(s))(K(s)-F(s))^{*} \mu(d s)\right) V(\ell)\right) \mu(d \ell) \\
& =\int_{\mathcal{S}} \operatorname{tr}(S(\ell) V(\ell)) \mu(d \ell) \\
& =\langle S ; V\rangle \leq\left\langle P-\mathcal{L}^{K}(P) ; V\right\rangle=\langle P ; V\rangle-\left\langle P ; \mathcal{T}^{K}(V)\right\rangle \\
& =\left(1-r_{\sigma}\left(\mathcal{L}^{K}\right)\right)\langle P ; V\rangle .
\end{aligned}
$$

Since $\langle P ; V\rangle \geq 0$ it follows from (A.27) that $r_{\sigma}\left(\mathcal{L}^{K}\right) \leq 1$. Suppose by contradiction that $r_{\sigma}\left(\mathcal{L}^{K}\right)=1$. From (A.27) one can conclude that

$$
\int_{\mathcal{S}} \alpha(s) \operatorname{tr}\left((K(s)-F(s))^{*} \mathcal{E}(V)(s)(K(s)-F(s))\right) \mu(d s)=0
$$

and, since $\alpha(s)>0$, it must be satisfied $\mathcal{E}(V)(s)^{1 / 2}(K(s)-F(s))=0$, that is, $\mathcal{E}(V)(s)^{1 / 2} K(s)=\mathcal{E}(V)(s)^{1 / 2} F(s)$. From this, one gets that $\mathcal{T}^{F}(V)=\mathcal{T}^{K}(V)=$ $r_{\sigma}\left(\mathcal{L}^{K}\right) V$, that is, $r_{\sigma}\left(\mathcal{L}^{K}\right) \in \sigma\left(\mathcal{T}^{F}\right)$. But since $1>r_{\sigma}\left(\mathcal{L}^{F}\right)=r_{\sigma}\left(\mathcal{T}^{F}\right)=\max \{|\lambda| ; \lambda \in$ $\left.\sigma\left(\mathcal{T}^{F}\right)\right\}$ it follows that $r_{\sigma}\left(\mathcal{L}^{K}\right)<1$ in contradiction with $r_{\sigma}\left(\mathcal{L}^{K}\right)=1$, completing the proof.

\section{A.2.3 Stationary Case}

Set $\Gamma^{s}=\left\{K \in \mathbb{H}_{1}^{p, n} \cap \mathbb{H}_{\text {sup }}^{p, n} ; K\right.$ stochastically stabilizes $(L, A)$ in the sense of Definition 5.2.4 . Recall the definition of $Y(V, k)$ in (A.21) and that $Y(k)$ in (A.11), (A.12) is such that $Y(k)=Y(V, k)$ when $Y(0)=V$. Next, the main result of the section is presented.

Theorem A.2.8. Suppose that Assumptions 3.2.2, 6.1.4 to 6.1.8 and 6.2.1 hold and that $(A, G)$ is stabilizable and $(L, A)$ is detectable. Then there exists a unique $Y \in \mathbb{H}_{1}^{n+} \cap \mathbb{H}_{\text {sup }}^{n+}$ satisfying (A.13)-(A.14), that is,

$$
Y=\mathcal{U}(Y, \pi)=\mathcal{L}(\mathcal{M}(Y, \pi), Y)+\mathcal{I}(\mathcal{M}(Y, \pi), 0, \pi) .
$$

Moreover $\mathcal{M}(Y, \pi) \in \Gamma^{s}$ and for any $V \in \mathbb{H}_{1}^{n+} \cap \mathbb{H}_{\text {sup }}^{n+}, Y(V, k) \rightarrow Y$ as $k \rightarrow \infty$. 
Proof. Let us initially show that there exists a sequence $Z(k) \in \mathbb{H}_{1}^{n+} \cap \mathbb{H}_{\text {sup }}^{n+}$ and a sequence $K(k) \in \Gamma^{s}, k=0,1, \ldots$, satisfying

$$
\begin{aligned}
Z(k) & =\mathcal{L}(K(k), Z(k))+\mathcal{I}(K(k), 0, \pi), \quad k=0,1, \ldots \\
K(k+1) & =\mathcal{M}(Z(k), \pi) .
\end{aligned}
$$

Let us show (A.29) by induction on $k$. From the fact that $(L, A)$ is detectable there exists $K(0) \in \Gamma^{s}$ that stochastically stabilizes $(L, A)$ in the sense of Definition 5.2.4, that is, such that $r_{\sigma}(\mathcal{L})<1$. Thus from Proposition A.2.6, Remark A.2.2 and Proposition 6.1.14 there exists a unique $Z(0) \in \mathbb{H}_{1}^{n+} \cap \mathbb{H}_{\text {sup }}^{n+}$ that satisfies $Z(0)=\mathcal{L}(K(0), Z(0))+\mathcal{I}(K(0), 0, \pi)$. Suppose now that the result holds for $k>0$. Define $K(k+1)=\mathcal{M}(Z(k), \pi)$. From Remark 6.1.15 it follows that

$$
Z(k)=\mathcal{L}(K(k), Z(k))+\mathcal{I}(K(k), 0, \pi)=\mathcal{L}(K(k+1), Z(k))+\mathcal{I}(K(k+1), W(k+1), \pi),
$$

where, for $s \in \mathcal{S}$,

$$
W(k+1, s)=(K(k+1, s)-K(k, s))\left(L(s) Z(k, s) L(s)^{*}+\pi(s) H(s) H(s)^{*}\right)(K(k+1, s)-K(k, s))^{*} .
$$

From Assumption 6.1.5 it follows that $H \in \widehat{\mathbb{H}}_{\text {sup }}^{q, p}$ and thus for some $\psi>0, H(\ell) H(\ell)^{*} \geq$ $\psi I$. Defining

$$
S(\ell)=\int_{\mathcal{S}} g(s, \ell) \pi(s) \psi(K(k+1, s)-K(k, s))(K(k+1, s)-K(k, s))^{*} \mu(d s),
$$

it follows that $S \in \mathbb{H}_{1}^{n+}$ and

$$
\mathcal{I}(K(k+1), W(k+1), \pi) \geq S,
$$

and thus combining (A.30) and (A.31) it results that

$$
Z(k)-\mathcal{L}(K(k+1), Z(k)) \geq S .
$$

From Proposition A.2.7 it follows that $K(k+1) \in \Gamma^{s}$ and from Proposition A.2.6 there exists a unique $Z(k+1) \in \mathbb{H}_{1}^{n+} \cap \mathbb{H}_{\text {sup }}^{n+}$ that satisfies

$$
Z(k+1)=\mathcal{L}(K(k+1), Z(k+1))+\mathcal{I}(K(k+1), 0, \pi),
$$

which proves the initial claim. It will be shown now the pointwise convergence of $Z(k) \rightarrow$ $Y \in \mathbb{H}_{1}^{n+} \cap \mathbb{H}_{\text {sup }}^{n+}$ and that $Y$ satisfies $Y=\mathcal{U}(Y, \pi)$. From (A.30) and (A.33) it results that $(Z(k)-Z(k+1))-\mathcal{L}(K(k+1), Z(k)-Z(k+1))=\mathcal{I}(K(k+1), W(k+1), \pi)-\mathcal{I}(K(k+1), 0, \pi) \geq 0$ and from Proposition A.2.6 it follows that $Z(k) \geq Z(k+1)$ as $(Z(k)-Z(k+1)) \in$ $\mathbb{H}_{1}^{n+} \cap \mathbb{H}_{\text {sup }}^{n+}$. This implies that there exists $Y \in \mathbb{H}_{1}^{n+} \cap \mathbb{H}_{\text {sup }}^{n+}$ such that $Z(k, \ell) \rightarrow Y(\ell)$ as $k \rightarrow \infty$ (see Proposition A.1.2, and notice that $Y \leq Z(0) \in \mathbb{H}_{1}^{n+} \cap \mathbb{H}_{\text {sup }}^{n+}$ ). Setting

$$
\begin{aligned}
\Psi(k, s)= & (A(s)+K(k, s) L(s)) Z(k, s)(A(s)+K(k, s) L(s))^{*} \\
& +\pi(s)\left((K(k, s) H(s))(K(k, s) H(s))^{*}+G(s) G(s)^{*}\right),
\end{aligned}
$$

it results that $\mathcal{L}(K(k), Z(k))(\ell)+\mathcal{I}(K(k), 0, \pi)(\ell)=\int_{\mathcal{S}} g(s, \ell) \Psi(k, s) \mu(d s)$ and from (A.34) and Remark 6.1.15 that

$$
0 \leq \Psi(k, s) \leq(A(s)+K(k, s) L(s)) Z(k-1, s)(A(s)+K(k, s) L(s))^{*}
$$




$$
\begin{aligned}
& +\pi(s)\left((K(k, s) H(s))(K(k, s) H(s))^{*}+G(s) G(s)^{*}\right) \\
& \leq A(s) Z(k-1, s) A(s)^{*}+G(s) G(s)^{*} \pi(s) \leq A(s) Z(0, s) A(s)^{*}+G(s) G(s)^{*} \pi(s) .
\end{aligned}
$$

From (A.35) one obtains that $0 \leq g(s, \ell)\|\Psi(k, s)\| \leq c\left(\|A\|_{\text {sup }}^{2}\|Z(0, s)\|+\|G\|_{\text {sup }}^{2} \pi(s)\right)$, and since $\int_{\mathcal{S}}\left(\|A\|_{\text {sup }}^{2}\|Z(0, s)\|+\|G\|_{\text {sup }}^{2} \pi(s)\right) \mu(d s)=\|A\|_{\text {sup }}^{2}\|Z(0)\|_{1}+\|G\|_{\text {sup }}^{2}$ it results from the dominated convergence theorem (see Hernández-Lerma and Lasserre, 2003, Section 1.5.2) and (A.29), after taking the limit as $k \rightarrow \infty$, that

$$
Y=\mathcal{L}(\mathcal{M}(Y, \pi), Y)+\mathcal{I}(\mathcal{M}(Y, \pi), 0, \pi)=\mathcal{U}(Y, \pi) .
$$

Set $K=\mathcal{M}(Y, \pi)$. It will be shown next that $K \in \Gamma^{s}$. According to Definition 5.2.3, $(A, G)$ stabilizable implies that for some $J \in \mathbb{H}_{\text {sup }}^{n, r}$ it holds that $r_{\sigma}(\mathcal{L})<1$, where $\mathcal{L}$ is as in (4.7) with $\Gamma(\ell)=A(\ell)+G(\ell) J(\ell), \ell \in \mathcal{S}$. Define

$$
\widehat{K}(\ell)=\left[\begin{array}{ll}
K(\ell) & 0
\end{array}\right], \widehat{F}(\ell)=\left[\begin{array}{ll}
0 & G(\ell)
\end{array}\right], \widehat{L}(\ell)=\left[\begin{array}{l}
L(\ell) \\
J(\ell)
\end{array}\right],
$$

and $\mathcal{L}^{\widehat{K}}$ as in $(4.7)$ with $\Gamma(\ell)=A(\ell)+\widehat{K}(\ell) \widehat{L}(\ell)=A(\ell)+K(\ell) L(\ell)$ and $\mathcal{L}^{\widehat{F}}$ as in $(4.7)$ with $\Gamma(\ell)=A(\ell)+\widehat{F}(\ell) \widehat{L}(\ell)=A(\ell)+G(\ell) J(\ell)$. Then $r_{\sigma}\left(\mathcal{L}^{\widehat{F}}\right)=r_{\sigma}(\mathcal{L})<1$ and from (A.36) it results that

$$
Y-\mathcal{T}_{1}^{\widehat{K}}(Y) \geq S
$$

where $S \in \mathbb{H}_{1}^{n+}$ is given by

$$
\begin{aligned}
S(\ell) & =\int_{\mathcal{S}} g(s, \ell) \hat{c} \pi(s)(\widehat{K}(s)-\widehat{F}(s))(\widehat{K}(s)-\widehat{F}(s))^{*} \mu(d s) \\
& =\int_{\mathcal{S}} g(s, \ell) \hat{c} \pi(s) K(s) K(s)^{*}+G(s) G(s)^{*} \mu(d s),
\end{aligned}
$$

for $\hat{c}<\min \{1, \psi\}$. Proposition A.2.7 implies that $r_{\sigma}\left(\mathcal{L}^{\widehat{K}}\right)=r_{\sigma}(\mathcal{L}(\mathcal{M}(Y, \pi), \cdot))<1$ and recalling Remark A.2.2 one gets that $K=\mathcal{M}(Y, \pi) \in \Gamma^{s}$. The goal now is to show the uniqueness of $Y \in \mathbb{H}_{1}^{n+} \cap \mathbb{H}_{\text {sup }}^{n+}$ satisfying (A.14). Suppose now that $\tilde{Y} \in \mathbb{H}_{1}^{n+} \cap \mathbb{H}_{\text {sup }}^{n+}$ satisfies

$$
\tilde{Y}=\mathcal{L}(\mathcal{M}(\tilde{Y}, \pi), \tilde{Y})+\mathcal{I}(\mathcal{M}(\tilde{Y}, \pi), 0, \pi)=\mathcal{U}(\tilde{Y}, \pi) .
$$

Repeating the same arguments as above one can conclude that $\mathcal{M}(\tilde{Y}, \pi) \in \Gamma^{s}$. Defining $W \in \mathbb{H}_{1}^{n+} \cap \mathbb{H}_{\text {sup }}^{n+}$ and $\tilde{W} \in \mathbb{H}_{1}^{n+} \cap \mathbb{H}_{\text {sup }}^{n+}$ as

$$
\begin{gathered}
W(\ell)=(\mathcal{M}(\tilde{Y}, \pi)(\ell)-\mathcal{M}(Y, \pi)(\ell))\left(L(\ell) Y(\ell) L(\ell)^{*}+\pi(\ell) H(\ell) H(\ell)^{*}\right) . \\
\cdot(\mathcal{M}(\tilde{Y}, \pi)(\ell)-\mathcal{M}(Y, \pi)(\ell))^{*} \\
\tilde{W}(\ell)=(\mathcal{M}(\tilde{Y}, \pi)(\ell)-\mathcal{M}(Y, \pi)(\ell))\left(L(\ell) \tilde{Y}(\ell) L(\ell)^{*}+\pi(\ell) H(\ell) H(\ell)^{*}\right) . \\
\cdot(\mathcal{M}(\tilde{Y}, \pi)(\ell)-\mathcal{M}(Y, \pi)(\ell))^{*},
\end{gathered}
$$

it results that

$$
Y=\mathcal{L}(\mathcal{M}(\tilde{Y}, \pi), Y)+\mathcal{I}(\mathcal{M}(\tilde{Y}, \pi), W, \pi)
$$




$$
\tilde{Y}=\mathcal{L}(\mathcal{M}(Y, \pi), \tilde{Y})+\mathcal{I}(\mathcal{M}(Y, \pi), \tilde{W}, \pi) .
$$

Subtracting (A.40) from (A.28) and (A.39) from (A.38) it is obtained respectively that

$$
(\tilde{Y}-Y)-\mathcal{L}(\mathcal{M}(Y, \pi), \tilde{Y}-Y)=\mathcal{I}(\mathcal{M}(Y, \pi), \tilde{W}, \pi)-\mathcal{I}(\mathcal{M}(Y, \pi), 0, \pi) \geq 0
$$

and

$$
(Y-\tilde{Y})-\mathcal{L}(\mathcal{M}(\tilde{Y}, \pi), Y-\tilde{Y})=\mathcal{I}(\mathcal{M}(\tilde{Y}, \pi), W, \pi)-\mathcal{I}(\mathcal{M}(\tilde{Y}, \pi), 0, \pi) \geq 0 .
$$

Recalling that $\mathcal{M}(Y, \pi)$ and $\mathcal{M}(\tilde{Y}, \pi)$ belong to $\Gamma^{s}$, from Proposition A.2.6 one can get that $0 \leq \tilde{Y}-Y \leq 0$, and thus $\tilde{Y}=Y$, showing that there is a unique solution for (A.14) as stated.

It remains to show that $Y(V, k) \rightarrow Y$ as $k \rightarrow \infty$ for any $V \in \mathbb{H}_{1}^{n+} \cap \mathbb{H}_{\text {sup }}^{n+}$. For $\ell \in \mathcal{S}$, define the sequence $\alpha(k, \ell)=\inf _{l \geq k} \pi(l, \ell)>0, k=0,1, \ldots$ such that $\alpha(k+1) \geq \alpha(k)>0$ and $R(k+1)=\mathcal{U}(R(k), \alpha(k)), R(0)=0$. From Proposition A.2.1 it follows that $\alpha(k) \rightarrow \pi$ as $k \rightarrow \infty$. It will be shown by induction on $k$ that

(i) $R(k) \leq R(k+1)$

(ii) $R(k) \leq Y(V, k) \leq Y(\mathcal{K}, V, k)$, where $\mathcal{K}=(\mathcal{M}(Y, \pi), \mathcal{M}(Y, \pi), \ldots)$.

For $k=0$ it follows that $R(0)=0 \leq R(1)$ and $R(0)=0 \leq V=Y(V, 0)=Y(\mathcal{K}, V, 0)$. Suppose that $R(k-1) \leq R(k)$. Then from Proposition A.2.5,

$$
R(k+1)=\mathcal{U}(R(k), \alpha(k)) \geq \mathcal{U}(R(k-1), \alpha(k-1))=R(k),
$$

showing (i). Assume now that $R(k) \leq Y(V, k)$. Then, again from Proposition A.2.5,

$$
R(k+1)=\mathcal{U}(R(k), \alpha(k)) \leq \mathcal{U}(Y(V, k), \pi(k))=Y(V, k+1) \leq Y(\mathcal{K}, V, k+1)
$$

showing (ii). From the fact that $r_{\sigma}(\mathcal{L}(\mathcal{M}(Y, \pi), \cdot))<1$ and from Proposition A.2.4 it follows from Lemma (L-1) in Kubrusly (1986) that $Y(\mathcal{K}, V, k) \rightarrow \tilde{Y} \in \mathbb{H}_{1}^{n+}$, where $\tilde{Y}$ satisfies $\tilde{Y}=\mathcal{L}(\mathcal{M}(Y, \pi), \tilde{Y})+\mathcal{I}(\mathcal{M}(Y, \pi), 0, \pi)$. Since $\mathcal{I}(\mathcal{M}(Y, \pi), 0, \pi) \in \mathbb{H}_{\text {sup }}^{n+}$ it follows from Proposition A.2.6 that $\tilde{Y} \in \mathbb{H}_{\text {sup }}^{n+}$, and thus $\tilde{Y} \in \mathbb{H}_{1}^{n+} \cap \mathbb{H}_{\text {sup }}^{n+}$. From Proposition A.2.6 and (A.28), $\tilde{Y}=Y$. Since $0 \leq R(k) \leq R(k+1) \leq Y(\mathcal{K}, V, k+1) \rightarrow Y$ it results, repeating the same arguments as presented above, that for some $R \in \mathbb{H}_{1}^{n+} \cap \mathbb{H}_{\text {sup }}^{n+}$, $R(k+1)=\mathcal{U}(R(k), \alpha(k)) \rightarrow R=\mathcal{U}(R, \pi)$ as $k \rightarrow \infty$. From the uniqueness established above, $R=Y$. Recalling that $R(k) \leq Y(V, k) \leq Y(\mathcal{K}, V, k)$ it follows that $Y(V, k) \rightarrow Y$ as $k \rightarrow \infty$, completing the proof of the theorem.

The next corollary is immediate from the results obtained in this appendix.

Corollary A.2.9. Consider $\mathcal{L}$ and $\mathcal{T}$ as defined in (4.7)-(4.8). If $r_{\sigma}(\mathcal{L})<1$ then for any $V \in \mathbb{H}_{1}^{n+} \cap \mathbb{H}_{\text {sup }}^{n+}$ there exists a unique $P \in \mathbb{H}_{1}^{n+} \cap \mathbb{H}_{\text {sup }}^{n+}$ such that

$$
P=\mathcal{L}(P)+V
$$

and for any $V \in \mathbb{H}_{\text {sup }}^{n+}$ there exists a unique $S \in \mathbb{H}_{\text {sup }}^{n+}$ such that

$$
S=\mathcal{T}(S)+V
$$

Proof. From Proposition A.2.6 it follows (A.41) and from Theorem A.1.6 it follows (A.42). 